\title{
Chaotic Dynamics in Networks of Spiking Neurons in the Balanced State
}

\author{
Dissertation \\ for the award of the degree \\ "Doctor rerum naturalium" \\ Division of Mathematics and Natural Sciences \\ of the Georg-August-University Göttingen
}

\author{
submitted by \\ Michael Monteforte
}

from Berlin

Göttingen 2011 
Thesis Committee:

- Prof. Dr. Fred Wolf (Reviewer)

Department of Nonlinear Dynamics,

Max Planck Institute for Dynamics and Self-Organization

- Prof. Dr. Theo Geisel (Reviewer)

Department of Nonlinear Dynamics,

Max Planck Institute for Dynamics and Self-Organization

- Prof. Dr. Eberhard Bodenschatz

Department of Fluid Dynamics, Pattern Formation, and Nanobiocomplexity,

Max Planck Institute for Dynamics and Self-Organization

Date of oral examination: 19th May 2011 


\section{Contents}

\begin{tabular}{llr}
\hline & Introduction & 1
\end{tabular}

1.1 The Balanced State of Cortical Networks _. . . . . . . . . . . . . . . 3

1.2 Single Neuron Dynamics $\ldots \ldots \ldots \ldots \ldots \ldots$

1.3 Chaotic Neural Network Dynamics $\ldots \ldots \ldots \ldots$

1.4 Lyapunov Exponents, Entropies, Dimensions _. . . . . . . . . . . . . . 11

1.5 Weak Pairwise Correlations in Strongly Correlated Network States . . . . . . . . 14

$\begin{array}{llr}2 & \text { Networks of Theta Neurons } & 17\end{array}$

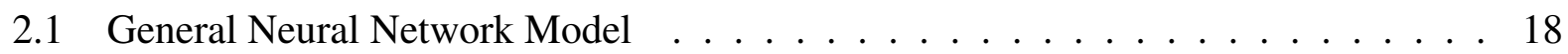

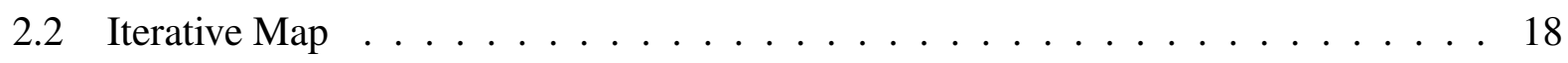

2.3 Single Spike Jacobian $\ldots \ldots \ldots \ldots$

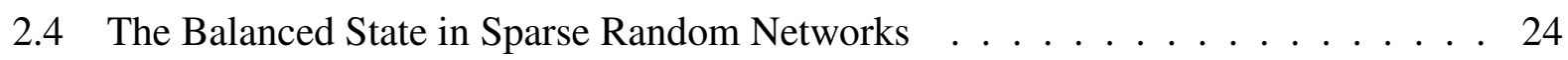

2.5 Networks of Theta neurons . . . . . . . . . . . . . . . . . . . . . 27

2.6 Asynchronous States and Transitions to Synchrony $\ldots \ldots \ldots \ldots$

2.7 Dynamics of Inhibitory Networks $\ldots \ldots \ldots \ldots \ldots \ldots$

2.8 Dynamics of Excitatory-Inhibitory Networks $\ldots \ldots \ldots \ldots$. . . . . . 41

2.9 Temporal Network Chaos . . . . . . . . . . . . . . . . . . . . . . . 44

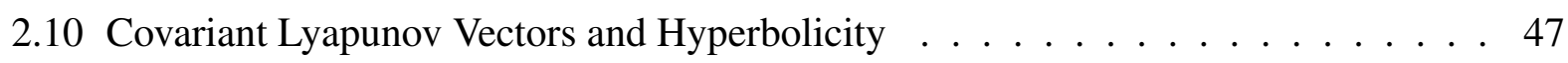

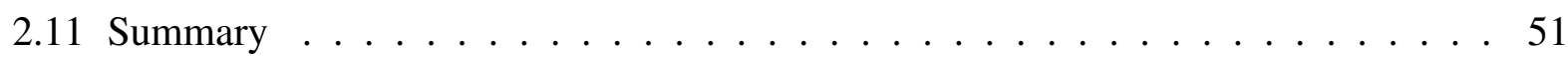

3 Networks of Rapid Theta Neurons 53

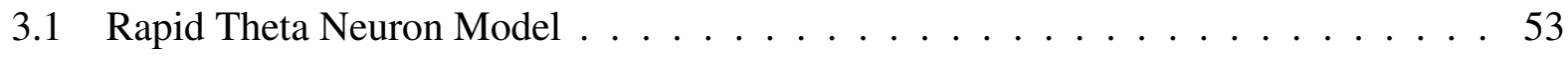

3.2 Balanced State in Rapid Theta Neuron Networks $\ldots \ldots \ldots$

3.3 Dynamics of Inhibitory Networks $\ldots \ldots \ldots \ldots$

3.4 Dynamics of Excitatory-Inhibitory Networks $\ldots \ldots \ldots 6$

3.5 Weak Pairwise Spike Correlations $\ldots \ldots \ldots \ldots$. . . . . . . . 73

3.6 Entangled Statistics $\ldots \ldots \ldots \ldots$

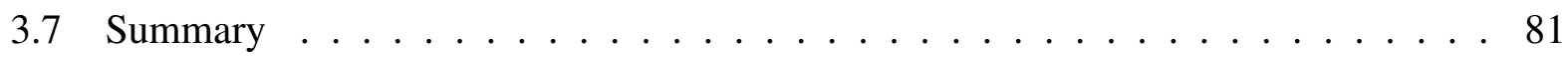


4 Networks of Correlated Leaky Integrate and Fire Neurons 83

4.1 Correlated Leaky Integrate and Fire Model . . . . . . . . . . . . . . . . . . . 83

4.2 Iterative Map $\ldots \ldots \ldots \ldots$

4.3 Single Spike Jacobian $\ldots \ldots \ldots$. . . . . . . . . . . . . . . 87

4.4 Balanced State in Correlated Leaky Integrate and Fire Networks . . . . . . . . . . 95

4.5 Dynamics of Inhibitory Networks $\ldots \ldots \ldots$. . . . . . . . . . . . . 97

4.6 Dynamics of Excitatory-Inhibitory Networks . . . . . . . . . . . . . . . 100

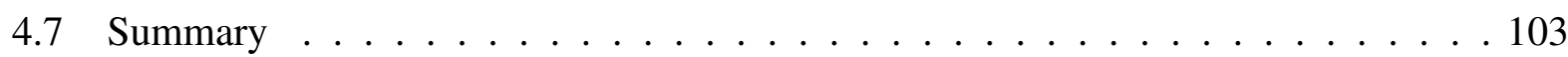

5 Stable Chaos in Networks of Leaky Integrate and Fire Neurons 105

$5.1 \quad$ Leaky Integrate and Fire Model . . . . . . . . . . . . . . . . . . . . . . 106

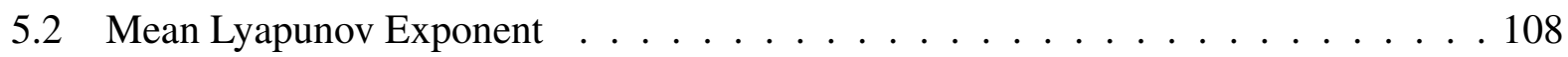

5.3 Stable Chaos in the Balanced State . . . . . . . . . . . . . . . . . . . . 112

$5.4 \quad$ Firing Rate Response After Single Spike Failures . . . . . . . . . . . . . . . . . 112

5.5 Exponential State Separation After Single Spike Failures ～. . . . . . . . . . . 115

5.6 Exponentially Separating Flux Tubes Around Stable Trajectories . . . . . . . . . . 116

5.7 Single Spike Perturbation in Rapid Theta Neuron Networks . . . . . . . . . . . . . 117

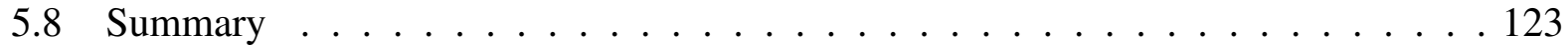

6 Discussion 125

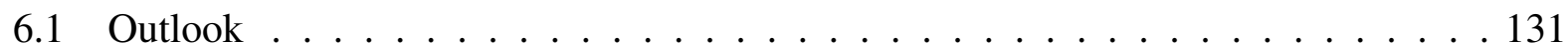

\begin{tabular}{|ll|}
\hline A Integrate and Fire Neuron Models & 135
\end{tabular}

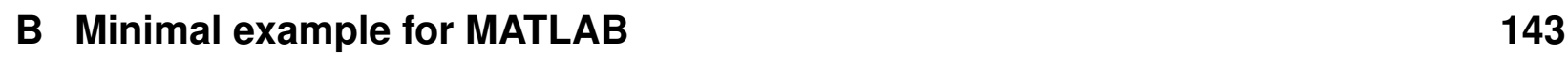

\begin{tabular}{lll}
\hline C Pairwise Spike Correlations & 145
\end{tabular}

\begin{tabular}{|lr}
\hline D Analytic Stability Analysis & 149
\end{tabular}

E Propagator Method 155

\begin{tabular}{ll}
\hline References & 157
\end{tabular}

\begin{tabular}{ll}
\hline List of Figures & 167
\end{tabular}

\begin{tabular}{lr}
\hline List of Symbols & 173
\end{tabular} 


\section{Introduction}

Unveiling the mystery of the brain is one of the most compelling fields of recent research. It has come a long way, dating back centuries and has made considerable progress especially in the last few decades. Although a great deal of detailed physiological and theoretical knowledge is now established, many fundamental questions still remain, and new ones arise with every step forward towards a better understanding of the functioning of the brain.

Information about the outside world is encoded by the sensory organs into electrical pulses and further sent to the brain. These almost unitary events called action potentials (AP) or simply spikes are the fundamental bits of information in the brain. They are processed in large networks of various types of neurons that are highly interconnected by different types of synapses. One purpose of this huge dynamical system is to extract, sort and store the incoming information and provide feedback to the outside world.

Finding the underlying principles of the information processing in the brain - the neural codeis the primary goal for a thorough understanding of the brain. An important aspect in this respect is the collective dynamics of neural networks, as they set the boundary conditions for the neural code. In this thesis, a novel approach for the theoretical characterization of the dynamical nature of neural networks is introduced. It is based on methods and tools from dynamical systems theory applied to models of spiking neuron networks.

One can easily think of three main factors that play a fundamental role in the dynamics of neural networks: (i) the individual neuron dynamics (e.g. details of the action potential generation), (ii) the individual synapse dynamics (e.g. action potential transmission) and (iii) the network topology (the detailed pathways of action potentials through the networks). The impact of all these factors on the network dynamics can be examined with the here introduced approach. It can directly be applied to arbitrary network topologies and different types of neurons described by their phaseresponse curves. An extension from synaptic $\delta$ pulse coupling to temporal synaptic transmission is discussed as well.

Particular emphasis is placed on the influence of the action potential generation in single neurons on the collective network dynamics. It was shown recently that cortical neurons exhibit a much sharper AP initiation than predicted from standard neuron models [1]. This fact, accompanied by controversial results of stable and chaotic dynamics in the balanced state of cortical networks depending on the single neuron dynamics [2-7], inspired an in-depth analysis of the effect of single neuron dynamics on the collective network dynamics.

The background of the controversy over the dynamical nature of the balanced state and what exactly the balanced state is will be discussed in the next section. This follows a brief summary of simple single neuron models and the relation of their action potential generation to that of real cortical neurons. A discussion of previous work on the analysis of neural network dynamics and the theoretical nonlinear dynamics background for the here-introduced approach is provided in Section 1.3 and 1.4 . The chapter is finished with a brief overview of spike correlations. 
In Chapter 2, the general approach for an exact characterization and quantification of the collective dynamics of spiking neuron networks is derived. This approach is based on exact closed-form expressions of the single neuron dynamics that allow us to run fast and precise event-based simulations and calculate the complete spectrum of Lyapunov exponents. As a first example, this approach is demonstrated in a comprehensive analysis of networks of theta neurons, the standard form of type I excitable membranes. These results establish that networks of such neurons exhibit extensive deterministic chaos. The derived rate of entropy production and attractor dimension are strikingly high. While such neural networks thus exhibit a huge repertoire of possible network states, information processing is restricted by the dynamical entropy production and appears to be limited to the immediate stimulus response. Parts of these results were published in Ref. [8].

The theta neuron model shares the low AP onset rapidness of other standard neuron models. To thoroughly investigate the influence of the much larger AP onset rapidness of real cortical neurons [1], we developed a new neuron model with variable action potential onset rapidness-the rapid theta neuron model. The collective network dynamics of such rapid theta neurons is comprehensively analyzed in Chapter 3 . The results demonstrate that the network dynamics strongly depends on the detailed dynamics of the action potential generation. For low AP onset rapidness, the results indicate qualitatively similar chaos as in theta neuron networks discussed in Chapter 2. For large AP onset rapidness, the network dynamics changes qualitatively and in the limit of instantaneous AP generation, the results indicate stable dynamics similar to leaky integrate and fire neuron networks (see Ref. [4-7] and Chapter 5). Between these contrary dynamics, we were able to quantify the edge of chaos depending on the network parameters. Towards the edge of chaos, with increasing AP onset rapidness of the neurons, the entropy production rate is strongly reduced, implying an enhanced capability for information processing. At the same time, the dimensionality of the network's attractor is reduced, reflecting the emergence of higher order correlations and entangled statistics in such models of cortical networks.

In Chapter 4, the introduced approach is extended to incorporate temporal synaptic transmission. Instead of $\delta$ pulse coupling as before, we analogously derive the approach for exponentially decaying postsynaptic currents in networks of leaky integrate and fire neurons. Our results show that such temporal postsynaptic currents can render the collective network dynamics chaotic. This chaos, however, is much weaker compared to the chaos in theta neuron networks with an active AP generation. Nevertheless, it shows that the stable dynamics of inhibitory leaky integrate and fire networks, referred to as stable chaos, is quite fragile.

To further investigate stable chaos in cortical networks and motivated by the recent experimental observation of high sensitivity of cortical networks to single spike perturbations, we studied finite size perturbations in inhibitory leaky integrate and fire networks, detailed in Chapter 5. Surprisingly, these networks, although exhibiting stable dynamics with respect to infinitesimal perturbations, are highly sensitive to the failure of individual spikes. Single spike failures lead to rapid decorrelation of the network's microstates which we quantified with a pseudo Lyapunov exponent. The pseudo Lyapunov exponent appears to increase to infinity in the large network and connectivity limit. This result of stable dynamics on the one hand and extremely unstable dynamics on the other hand bridges the gap between the previously contradictory results of binary and leaky integrate and fire neuron networks [2-7]. The analysis of finite size perturbations clarifies that these opposed results arise depending on the order in which the weak perturbation limit and the large network limit are taken. Our analysis also reveals the complex structure of the underlying phase space, which is composed of tangles of exponentially separating flux tubes around unique stable trajectories. 


\subsection{The Balanced State of Cortical Networks}

The cerebral cortex plays a key role in information processing in the mammalian brain. Surprisingly, cortical neurons embedded in intact cortical circuits emit action potentials (spikes) in a seemingly random fashion [10,11]. The collective state of cortical neurons is thus described as an asynchronous irregular stateasynchronous in terms of the firing activity of different neurons across the network, and irregular in terms of the firing activity of individual neurons over time. The prevailing hypothesis of the origin of this asynchronous, irregular state is a dynamic balance between excitation and inhibition called the balanced state of cortical networks [2, 3, 12, 13].

The balance of excitation and inhibition has been demonstrated experimentally in vitro and in vivo in various parts of the nervous system, for example in the cortex [14-17], the hippocampus [18], the superior colliculus [19], the brain stem [20] and even the spinal cord [21]. As the Zhuangzi from 300 BC claims: "Perhaps this is the law of everything."

Cortical neurons are permanently bombarded with synaptic inputs. A large fraction of these inputs, however, are received from neurons of different brain areas, and can be assumed to be un-

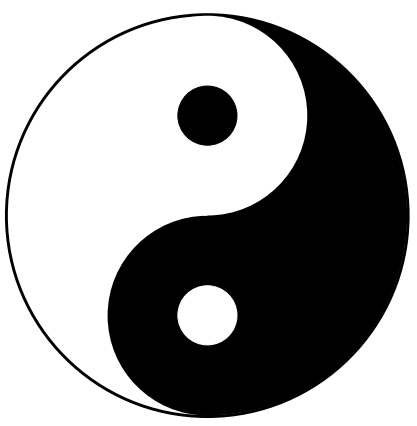

Yin and Yang: "Yin in its highest form is freezing while Yang in its highest form is boiling. The chilliness comes from heaven while the warmness comes from the earth. The interaction of these two establishes $\mathrm{He}$ (harmony), so it gives birth to things. Perhaps this is the law of everything yet there is no form being seen."

Zhuangzi, Ch. $21[9]$ correlated. It thus results in a temporally constant input to the neurons. The temporally constant input would lead to periodic regular firing of the neurons, as the spike generating process was found to be highly reliable [22]. The observed irregular firing is therefore thought to arise from fluctuations in the synaptic inputs [23]. Interestingly, these fluctuations are the result of a cooperative network effect due to the dynamic balance of excitation and inhibition [2, 3].

A steady state with finite average firing rate in a recurrent neural network requires a balance of excitation and inhibition. If all neurons were excitatory coupled, the positive feedback would quickly lead to an explosion of the firing rates. Thus the excitation must be compensated by inhibition. This dynamically drives the network into a responsive state at a low average firing rate. A great deal of theoretical work has been devoted to the characteristics of this balanced state and has established that it emerges robustly without the need for fine-tuning of parameters or stochastic sources in sparse random networks with relatively strong synapses [2, 3, 5, -7, 24-33]. Relatively strong synapses yield large input fluctuations of the same order of magnitude as the mean current, which is important in explaining the irregular activity. A possible correlation in the inputs would then lead to a synchronized state. To rule out this possibility, it was originally necessary to consider sparse networks in which neurons are expected to be uncorrelated [34].

More recently it was shown that the balanced state also emerges dynamically in dense networks [32,33]. In dense networks, the number of synapses per neuron increases proportional to the network size, the connection probability held constant. In sparse networks, the number of synapses per neuron is held constant with increasing network size. The emergence of the balanced state in sparse networks is due to weak correlations. In dense networks, the current correlations in the excitatory and in the inhibitory populations are positive and large. Because the inhibitory population tracks the excitatory population with a small time lag (vanishing in the large network 
A

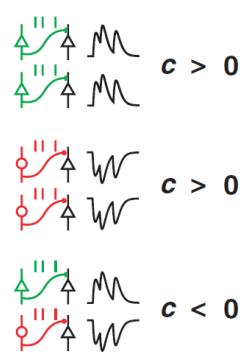

B

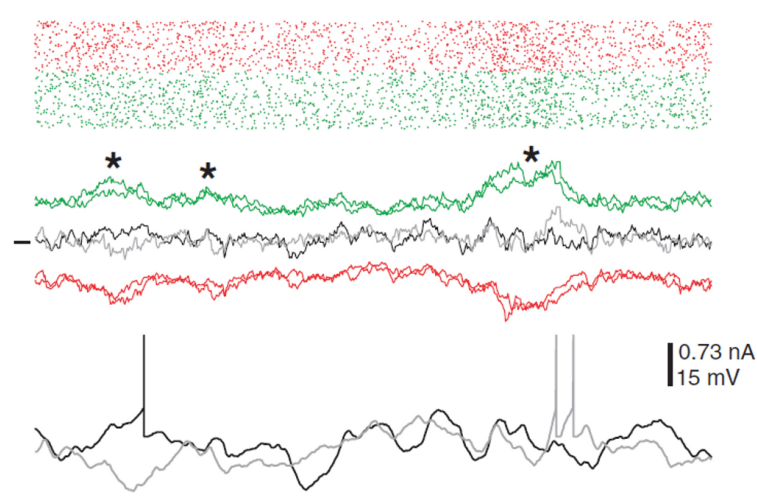

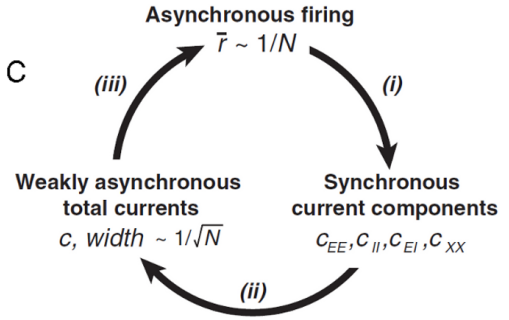

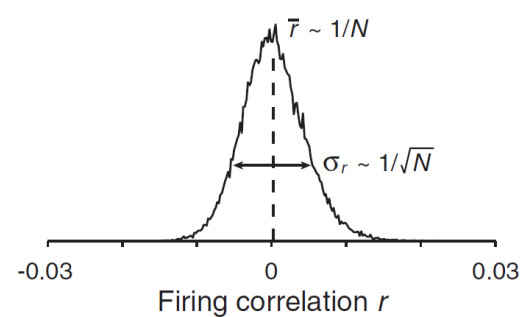

Figure 1.1 - The balanced state in densely connected networks. (Figures from Ref. [32]) (A) Shared inputs from either excitatory or inhibitory neurons contribute positively to the input current correlation $c$ but correlations between excitatory and inhibitory inputs contribute negatively (green: excitatory, red:inhibitory). (B) The balance of input correlations leads to the asynchronous irregular state in densely connected networks (spike patterns (top), synaptic currents (middle) and membrane potentials (bottom)), asterisks mark cancellation of simultaneous fluctuations in excitatory and inhibitory input current. (C) Selfconsistent circle of correlations leading to the asynchronous balanced state (top) and weak but broadly distributed pairwise spike correlations (bottom).

limit), the positive excitatory and negative inhibitory currents are negatively correlated and balance the positive current correlations [32]. Thus, in dense networks, positive and negative current correlations are dynamically balanced, leading to the asynchronous irregular network state with low spike correlations (Fig. 1.1).

A question arising is what are the advantages of the asynchronous irregular balanced state. There are several reasons. It emerges without fine-tuning and can thus easily adapt to different scenarios. An increase in the external input leads to a controlled increase of the firing rate. The tracking of small input fluctuations is important in separating the information from the input streams. It is unlikely that information is provided by large fluctuations in the overall input, but rather is encoded in small variations of a few inputs. Fast tracking of small input variations is a key feature of the balanced state [3, 24, 32] and certainly fundamental for the performance of cortical networks. The likely chaotic nature of the balanced state would be beneficial for this process but also implies constraints on the neural code due to a dynamic entropy production.

It is therefore of fundamental importance for the understanding of information processing to clarify whether the balanced state exhibits chaotic or stable dynamics. A network with chaotic dynamics exhibits sensitivity to initial conditions. On the one hand, this is beneficial for distinguishing small differences in the network's microstate that reflect variations of the input stream. On the other hand, due to the sensitivity, information is also gained about irrelevant microscopic processes such as stochastic ion channel openings. This produces entropy that overwrites the actual input information provided to the network and thus limits information processing. Another advantage of chaotic dynamics is the permanent exploration of different states on the chaotic attractor without getting locked into spurious states. This could in principle occur in the case of stable dynamics. A network with stable dynamics is not sensitive to initial conditions. The input information provided to the network is thus not degraded over time by a dynamic entropy production. 
It would also be more noise-resistant, but at the potential cost of possibly untracked changes of small input variations and possibly long transients after strong input variations. These two opposing scenarios, stable or chaotic dynamics, require completely different strategies for information processing. Therefore, one can conclude that the dynamical nature of cortical networks sets the boundary conditions of the underlying neural code.

Previous studies have shown that the dynamical nature of the balanced state strongly depends on the neuron model and network architecture used. Originally, the balanced state was reported to be strongly chaotic [2,3]. This strong chaos, characterized by an infinite largest Lyapunov exponent, was found in excitatory-inhibitory networks of binary neurons. In contrast, balanced inhibitory networks of leaky integrate and fire neurons were demonstrated to generally exhibit a peculiar form of stable dynamics in the limit of vanishing synaptic time constants [5-7]. This type of dynamics, characterized by a negative largest Lyapunov exponent despite an irregular network activity shares similarities with a type of aperiodic dynamics termed stable chaos [35]. It seems to be robust in balanced leaky integrate and fire networks, in the sense that small changes in the network model, e.g., adding a few excitatory neurons, does not destabilize the network dynamics [7]. Including a finite decay time of synaptic currents, however, turns inhibitory leaky integrate and fire networks chaotic in the conventional sense of positive Lyapunov exponents [5]. This will also be addressed in Chapter 4 The leaky integrate and fire neuron and the binary neuron models, however, lack the active spike-generating mechanism of real neurons and physiologically detailed neuron models. The influence of this single neuron instability on the collective network dynamics will be investigated in detail in Chapters 2 and 3 .

\subsection{Single Neuron Dynamics}

Neurons are highly interconnected cells in the brain. They are composed of dendrites, the cell body called the soma and an axon. The connections between axons and dendrites of different neurons are established at synapses. Information is communicated in terms of electrical pulses, called action potentials or spikes. The neurons receive electrical signals through their tree of dendrites and send out action potentials to other neurons through their axon. The detailed dynamics of action potential generation plays a central role in the information processing of neural networks as will be worked out in this thesis.

Action potentials are short events of fast depolarization of the neuron's membrane potential. We will give a very simplified description of an action potential generation. The cell membrane of a neuron is a phospholipid bilayer that is impermeable to ions. It can be thought of as a capacitor, insulating

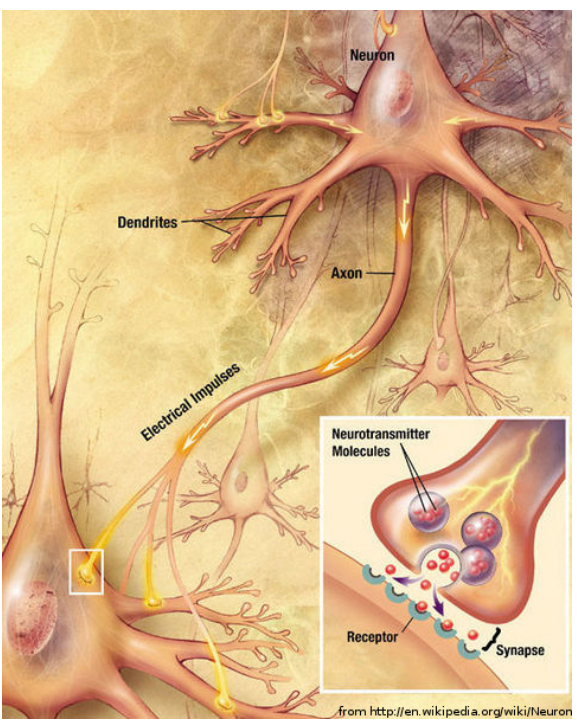
the inside of the cell from the outside. The most important ions involved in the generation of an action potential are sodium $\mathrm{NA}^{+}$and potassium $\mathrm{K}^{+}$. The difference of the ion concentration between the inside and the outside of the cell defines the voltage across the membrane and is usually referred to as the membrane potential relative to the outside of the cell. A typical resting potential of a neuron is $-60 \mathrm{mV}$. If the membrane potential becomes depolarized, voltage-gated ion-specific channels are triggered. They are complex proteins embedded 
in the cell membrane and responsible for the generation of action potentials. At a certain threshold of the membrane potential, typically around $-50 \mathrm{mV}$, fast sodium channels open abruptly, leading to an influx of sodium ions into the cell. This depolarizes the membrane further and more channels open, leading to an overshoot of the membrane potential. The sodium channels then deactivate and the slower potassium channels open. They induce an outward potassium current that hyperpolarizes the membrane potential and terminates the overshoot. This short overshoot in the membrane potential time course is called an action potential. The action potential is generated at the axon hilloc and then propagates down the axon as the inward currents provoke action potentials in the adjacent sections of the membrane.

Neuron models describe the membrane potential in terms of differential equations of the membrane potential, or voltage. The membrane acts as a capacitor with voltage dependent currents. The governing equation of the membrane potential $V$ is

$$
C \frac{\mathrm{d} V}{\mathrm{~d} t}=I_{\mathrm{m}}+I_{S}
$$

with the membrane capacitance $C$, the voltage dependent membrane current $I_{m}$ and the synaptic input current $I_{s}$. Different neuron models differ in the details of the membrane current $I_{m}$. Generally, they are written down in the form

$$
I_{\mathrm{m}}=-\sum_{i} g_{i}\left(V-E_{i}\right)
$$

where the sum runs over all types of ion channels to be considered, $g_{i}$ is the conductance of the ion channel and $E_{i}$ is its reversal potential. The number and types of ion channels depend on the type of neuron and the level of physiological detail of the neuron model. Important to mention here is the Hodgkin-Huxley model, the first model to quantitatively describe action potential generation [36]. Developed to describe action potential generation in the giant squid axon, this fundamental model incorporates a leakage, sodium and potassium current and is still the standard for the explanation of action potential generation. Hodgkin and Huxley received the "Nobel Prize in Physiology or Medicine" in 1963 for this breakthrough in biophysics. For the description of cortical neurons, however, it might be less suitable because it exhibits type II firing.

Neuron models are divided into two main types, type I and type II (Fig. 1.2). They are distinguished by the relationship between the injected current $I$ and their firing rate $F$ captured in the FI-curve. Type I excitable neurons are characterized by a continuous onset of firing above a certain current threshold $I_{T}$. This behavior is associated with a saddle node on invariant circle (SNIC) bifurcation $F \propto \sqrt{I-I_{T}}$ or a homoclinic bifurcation $F \propto 1 / \log \left(I-I_{T}\right)$. Type II excitable neurons are characterized by a discontinuous onset of firing, a jump in the $F I$-curve. This behavior is associated with a Hopf-bifurcation.

The Hodgkin-Huxley model is a type II model, but the majority of cortical neurons are type I excitable neurons. Connor and Stevens modified and extended the Hodgkin-Huxley model such that their model would reproduce the type I behavior of gastropod neurons [39]. In addition to the sodium and potassium currents, they included a transient potassium current called A-current. Another important standard model is the Wang-Buzsáki model developed to describe interneurons in the hippocampus [38]. It is a type I model without the need of an additional A-current and the close association of the hippocampus with the cerebral cortex makes it quite suitable for cortical neurons. We will, however, discuss below that cortical neurons exhibit a much faster action potential onset than predicted by these and other standard models. 

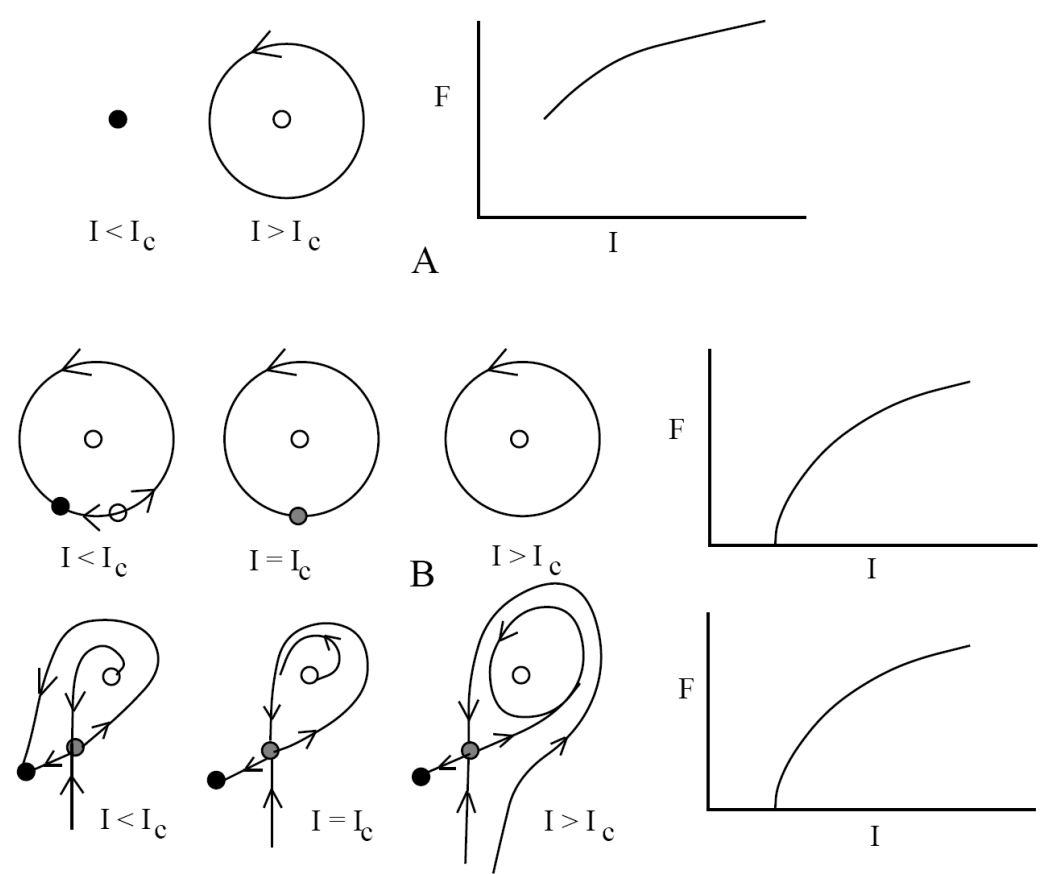

$\mathrm{C}$

Figure 1.2 - Firing rate functions of type I and type II excitable membranes. (Figures from Ref. [37]) (A) Type II, subcritical Hopf bifurcation. (B) Type I, saddle-node limit cycle bifurcation. (C) Type I, regular homoclinic bifurcation. (Full circles are stable, open circles are unstable nodes, and grey circles are saddles)

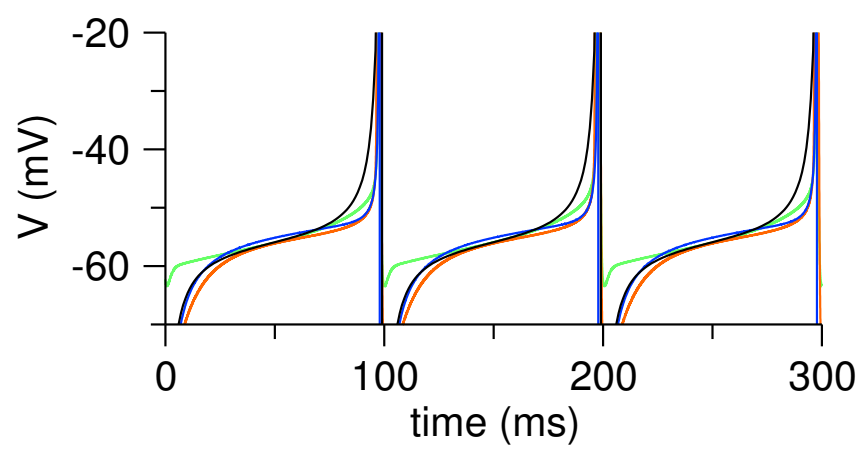

- Connor-Stevens model

- Wang-Buzsàki model

- Rapid theta neuron model $(\mathrm{r}=3)$

- Theta neuron model $(\mathrm{r}=1)$

Figure 1.3 - Standard neuron models of noncortical neurons. Standard neuron model such as the WangBuzsáki model [38] and the Connor-Stevens model [39] exhibit low AP onset rapidness. They are compared with the theta neuron model and the rapid theta neuron model with AP onset rapidness $r=3$. 
In computational neuroscience, another class of neuron models is very important which are physiologically less realistic but can quantitatively well-describe the action potential generation. These are the integrate and fire models: the perfect integrator (PIF) where the membrane current is zero, the leaky (also linear) integrate and fire model (LIF) where only a leak current is considered, the quadratic integrate and fire model (QIF) where the membrane current is a quadratic function of the voltage, and the exponential integrate and fire model (EIF) where a leak term and an exponential function constitute the membrane current. The theta neuron model is a phase representation of the QIF model and will be described in more detail in Section 2.5. To study the effect of rapid action potential onset on the network dynamics we will introduce another one-dimensional neuron model, the rapid theta neuron model with variable spike onset rapidness in Section 3.1. A comparison with the conductance-based Connor-Stevens and the Wang-Buzsáki models is shown in Fig. 1.3. It can be seen that these neuron models exhibit a rather slow spike onset rapidness corresponding to an $r$ between 1 and 3. A comparison with the EIF model and more details on the integrate and fire models are provided in Appendix A.

\subsubsection{Action Potential Generation in Cortical Neurons}

Cortical neurons were found to exhibit an action potential (AP) generating mechanism, that could not be reproduced by Hodgkin-Huxley type models [1]. They exhibit a much sharper AP onset than predicted from standard single compartment neuron models (Fig. 1.4(a)). An intriguing explanation of this sharper depolarization is that the positive feedback between the opening of sodium channels and the increased depolarization through the inward flux of sodium is additionally boosted by a cooperative opening of adjacent sodium channels [1]. Although a fascinating idea, questioning the supposedly well-understood process of AP initiation in neurons has triggered a heated debate [41,42].

Another explanation for the observed discrepancy between the AP generation in standard models and in real cortical neurons is the backpropagation of the spike from its site of initiation at the axon initial segment (AIS) into the soma [40]. The experiments reported in Ref. [40], however, confirm that the AP onset rapidness is already strongly increased in the AIS (Fig. 1.4(b)). As demonstrated with a multicompartment model, it further increases due to the backpropagation into the soma, but the initial high AP onset rapidness in the AIS could only be obtained with a high sodium density ten times larger in the AIS than in the soma [40]. While the backpropagation hypothesis is supported by different types of sodium channels in the AIS [43], the assumed sodium density for the initial sharp AP generation seems unphysiologically high. Recent fluorescence imaging revealed that this ratio is maximally three to one [44]. The origin of sharp AP onset thus still remains an open and interesting question.

Here, we will discuss the influence of the AP onset rapidness on the collective network dynamics. The developed rapid theta neuron model allows us to vary the AP onset rapidness arbitrarily. In the rapid theta neuron model, the AP onset rapidness is measured relative to the membrane time constant $\tau_{\mathrm{m}}$. At the resting potential, the rapidness is $-1 / \tau_{\mathrm{m}}$ and at the AP onset it is $r$ times larger, namely $r / \tau_{\mathrm{m}}$. For noncortical neuron models, we can see in Fig. 1.3 that $r$ is in the range 1 to 3 . From the experiments in cortical neurons by Yu et al. [40] and Naundorf et al. [1], we can estimate the slope at AP initiation to be between $7 \mathrm{~ms}^{-1}$ and $20 \mathrm{~ms}^{-1}$. Thus following the current literature and assuming a membrane time constant of $\tau_{\mathrm{m}}=10 \mathrm{~ms}$ which will be used throughout this thesis, the AP onset rapidness $r$ of cortical neurons lies in the range of 10 to 100 . In our analysis of the effect of the AP onset rapidness on the collective network dynamics in Chapter 3, we will thus 

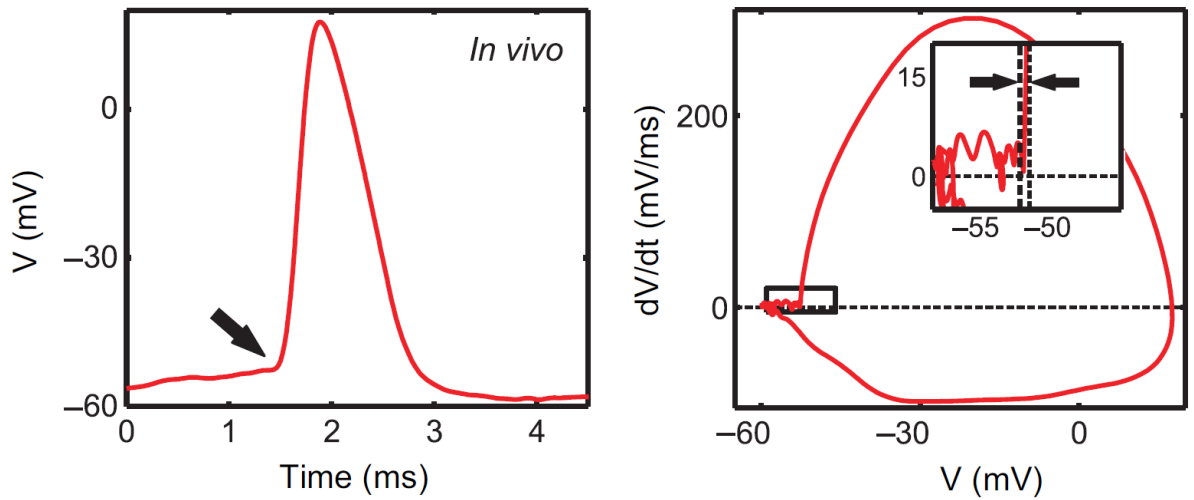

(a) (Figures from Naundorf et al. [1]) left: voltage trace of a cortical neuron in vivo showing the characteristic kink at action potential onset, right: phase plot of the action potential.
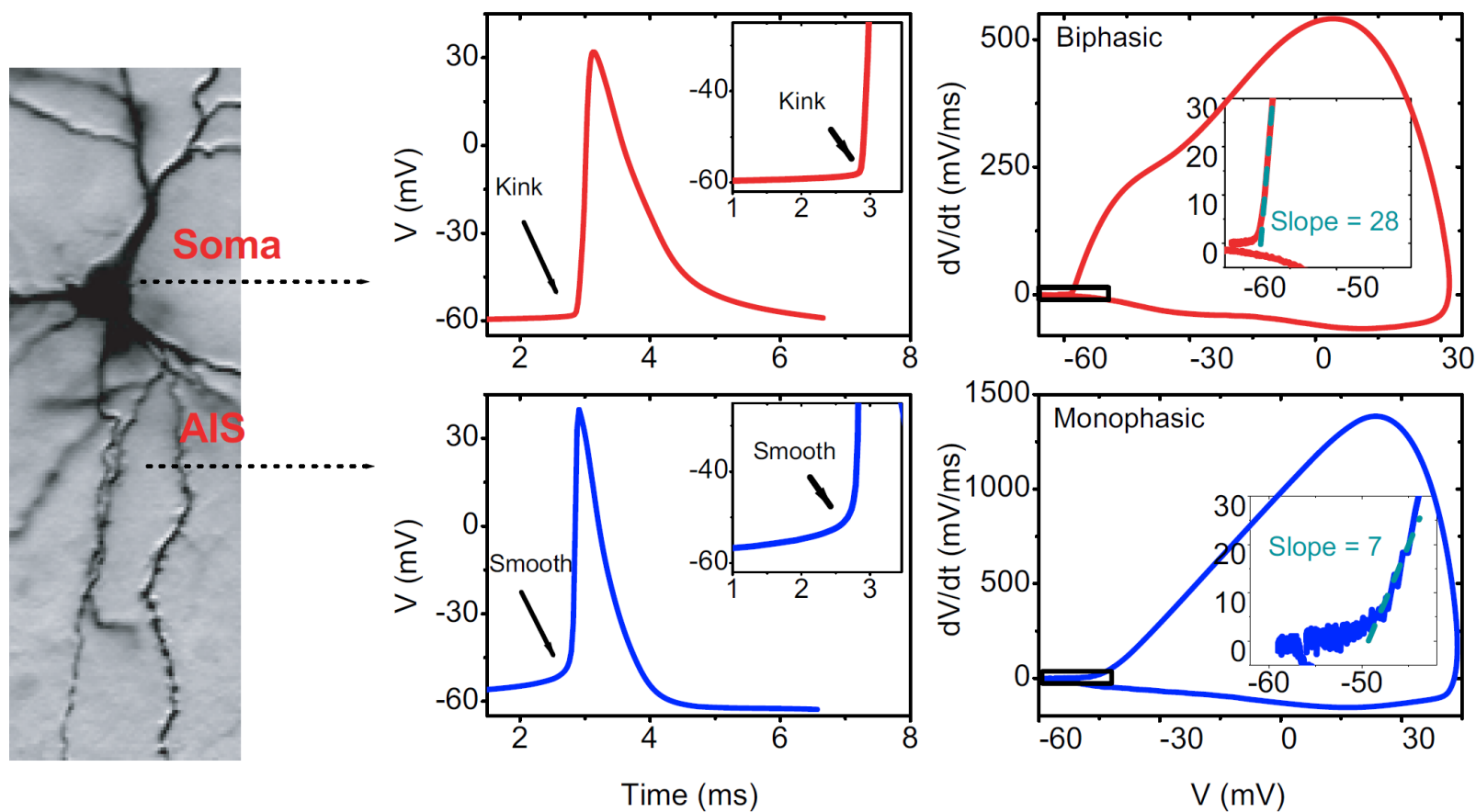

(b) (Figures from Yu et al. [40]) left: cortical layer 5 pyramidal cell, middle: voltage trace recorded in the soma (top) and in the axon initial segment (bottom), right: phase-plot of the action potentials.

Figure 1.4 - Action potential generation in cortical neurons. They exhibit a very large AP onset rapidness in the soma and at the sight of initiation in the axon initial segment. 
mostly use the representative values $r=1,3,10,100$.

\subsection{Chaotic Neural Network Dynamics}

Much theoretical work has been devoted to the characteristics of the emerging firing patterns of neural networks, leading to important implications and insight towards an understanding of the neural code. Studying the dynamical nature of neural networks has always been an important aspect in this endeavor. The idea of chaotic dynamics in neural circuits is not new per se [45] and its detailed investigation has been approached several times.

The first study of high dimensional neural networks in a mean-field description showed a direct transition from a stationary to a chaotic state [46]. This transition depended on the gain parameter of the nonlinear input-output function of the single neurons. The authors of this study already surmised that the number of positive Lyapunov exponents would scale proportional to the system size. Analogous to spin glasses, the connectivity in the networks was assumed to be dense and random but asymmetric. Biologically more realistic is a network topology with an excitatory and inhibitory population of neurons. Then, Dale's law, stating that a neuron always sends either excitatory or inhibitory signals independent of target neuron is fulfilled. The dynamics of such excitatory-inhibitory networks of binary neurons in the balanced state was investigated in a seminal mean-field analysis and found to be strongly chaotic [2,3]. The extreme form of chaos in such networks was characterized by an infinite Lyapunov exponent. The authors ascribe this to the discreteness of the degrees of freedom due to the binary neuron model used because it implies an infinitely high gain of microscopic changes. A related work studied networks of conductancebased neurons with a spatially structured connectivity as a model of a hypercolumn in the visual cortex [47]. Unlike networks in the balanced state, this network resulted in correlated synaptic inputs leading to a synchronous but temporally irregular state. This state was also characterized by chaotic dynamics. In a different approach, based on an abstract dynamical system representation for neural networks, it was proposed that cortical networks are quite generally chaotic and that reproducible precise spike timing in cortical networks is unlikely [48, 49].

A recent series of studies based on leaky integrate and fire neurons and their generalizations reported various phenomena in the collective dynamics that strongly depended on the networks' details and require an analysis of the network dynamics keeping track of every individual spike [50-59]. Globally coupled networks of leaky integrate and fire neurons for example show rapid convergence to stable periodic spike sequences when inhibitory coupling is dominant [60]. In diluted inhibitory networks of leaky integrate and fire neurons, the synchronous state is still stable and the time to synchronization obeys a speed limit, depending on the topology of the networks [61-63]. Diluted leaky integrate and fire networks with excitatory coupling on the other hand show long and chaotic transients [64], and allowed for an analytical approximation of the largest Lyapunov exponent. This was recently complemented by numerical results for fully coupled networks with time dependent stimuli [65]. Diluted leaky integrate and fire networks with inhibitory coupling also show long transients, but are characterized by stable dynamics [4-7]. It was further suggested that leaky integrate and fire networks with excitatory and inhibitory populations exhibit high dimensional chaos but the time series analysis used was inconclusive in this case [66].

The approach introduced in this thesis provides a controlled method beyond the calculation of the largest Lyapunov exponent, for the characterization of spiking neuron network dynamics. Based on exact solutions of the single neuron dynamics, it allows for the numerically exact calcula- 
tion of the full spectrum of Lyapunov exponents, providing detailed information about the network dynamics.

\subsection{Lyapunov Exponents, Entropies, Dimensions}

A dynamical system can be described by differential equations:

$$
\frac{\mathrm{d} \vec{x}}{\mathrm{~d} t}=\vec{F}(\vec{x}), \quad \vec{x} \in \mathbb{R}^{N}
$$

where $\vec{x}$ describes the state of the system, $t$ denotes time and $\mathbb{R}^{N}$ is called the phase space (also state space). The solutions of (1.1) given an initial condition are called trajectories and typically settle after a transient on a subset of the phase space called the attractor. This can be a fixed point with zero-dimension, a periodic orbit with one-dimension, a quasi-periodic orbit with integer dimension (torus) or a strange attractor with chaotic dynamics and fractal dimension. In chaotic systems, the sensitive dependence on initial conditions leads to exponentially separating trajectories. This stretching of phase-space volume in some directions is accompanied by contraction in other directions, such that the overall volume is preserved (conservative systems) or compressed (dissipative systems as in the following). Folding of the stretched trajectories leads to the fractal, self-similar structure of strange attractors and assures that it is bounded. Given the attractor being compact and invariant under the dynamical system, there exists an invariant probability measure with compact support on the attractor [67]. This measure can be chosen to be ergodic, call it a physical measure (e.g. Kolmogorov measure, SRB measure), which has far-reaching consequences for studying such dynamical systems.

Ergodic theory allows us to study the long-term behavior of a dynamical system using the ergodic measure as it represents time averages. The ergodic theorem states that the time average of a continuous function $\varphi$ can be substituted by the average with respect to the measure $\rho$ for almost all initial conditions:

$$
\lim _{t \rightarrow \infty} \frac{1}{t} \int_{0}^{t} \varphi\left(x\left(t^{\prime}\right)\right) \mathrm{d} t^{\prime}=\int \varphi(x) \rho(\mathrm{d} x) .
$$

Crucial in the analysis of the dynamics is the sensitivity to initial conditions. A system exhibits sensitivity to initial conditions if the distance between two originally infinitesimal close points $\vec{x}_{1}$ and $\vec{x}_{2}=\vec{x}_{1}+\varepsilon \delta \vec{x}$ grows exponentially. The time evolution of the infinitesimal perturbation $\varepsilon \delta \vec{x}$ with strength $\varepsilon \rightarrow 0$ and normalized direction $\delta \vec{x}$ is given by the solution $\vec{x}(t)$ of Eq. (1.1). In linear order of the perturbation size, one gets $\vec{x}_{2}(t) \approx \vec{x}_{1}(t)+\varepsilon \mathbf{T}_{t} \delta \vec{x}$ with the derivative $\mathbf{T}_{t}=$ $\mathrm{d} \vec{x}(t) / \mathrm{d} \vec{x}(0)$ also called the Jacobian matrix. The Jacobian matrix thus determines the growth rate of infinitesimal perturbations. The average exponential growth rate is captured by the Lyapunov exponent $\lambda=\lim _{t \rightarrow \infty} \frac{1}{t} \log \left\|\mathbf{T}_{t} \boldsymbol{\delta} \vec{x}\right\|$.

Lyapunov exponents are the centerpiece in our investigation of the dynamics of spiking neuron networks. They capture the exponential growth rate of infinitesimal perturbations. This, however, depends on the direction of the perturbation $\delta \vec{x}$. Therefore, in an $N$-dimensional system there exists a spectrum of $N$ Lyapunov exponents $\lambda_{1} \geq \ldots \geq \lambda_{N}$. The largest Lyapunov exponent $\lambda_{1}$ plays an important role in characterizing the dynamical nature of a system, as it determines the growth of perturbations applied in a random direction (see Eq. (1.4)). If perturbations grow exponentially (positive largest Lyapunov exponent) the system displays a sensitive dependence to initial conditions and is considered to be chaotic. If perturbations decay exponentially (negative 
largest Lyapunov exponent) the system is called stable. The full spectrum of Lyapunov exponents captures additional important information about the dynamics of the system.

The foundation of the existence and the computation of the complete spectrum of Lyapunov exponents builds the multiplicative ergodic theorem, also known as Oseledec theorem [67]. Briefly, it states that for the product of matrices $\mathbf{T}_{t}=\mathbf{D}_{s} \cdots \mathbf{D}_{0}$ with $s \leq t$ the Oseledec matrix $\Lambda$ exists for almost all initial conditions:

$$
\Lambda=\lim _{t \rightarrow \infty}\left(\mathbf{T}^{*} \mathbf{T}\right)^{\frac{1}{2 t}}
$$

where $\mathbf{T}^{*}$ denotes the transpose of $\mathbf{T}$ and the logarithms of the eigenvalues of the Oseledec matrix (1.3) are the Lyapunov exponents $\lambda_{1} \geq \cdots \geq \lambda_{N}$. However, because of the ill-conditioning of the Oseledec matrix (1.3), due to the exponential growth and decay of the eigenvalues, the standard procedure for the numerical calculation of the Lyapunov exponents [68, 69] exploits a different part of the Oseledec theorem. Namely, the fact that the growth rate of a volume element spanned by vectors of different subspaces is given by the sum of the corresponding Lyapunov vectors. The exact Lyapunov exponents are equivalently defined for almost all initial conditions by the limits:

$$
\lambda_{i}=\lim _{t \rightarrow \infty} \frac{1}{t} \log \|\mathbf{T} \delta \vec{x}\| \quad \text { if } \delta \vec{x} \in E_{i} \backslash E_{i+1},
$$

where the $E_{i}$ 's are a valid decomposition of the phase space corresponding to subspaces spanned by the eigenvectors associated to the eigenvalues $\leq \exp \left(\lambda_{i}\right)$ of the Oseledec matrix. The vectors $\delta \vec{x}$ are normalized vectors in the corresponding subspace $E_{i}$. In particular, Eq. (1.4) means that for almost all vectors, namely $\delta \vec{x} \notin E_{2}$, the limit is the largest Lyapunov exponent. Even though the multiplicative ergodic theorem at first sight seems less strong than the ergodic theorem, the existence of the limit is remarkable since it deals with non-commuting matrices. Another important point is that the Lyapunov exponents are topological invariant. Thus, one can say that they do not change under moderate changes of coordinates.

The full spectrum of Lyapunov exponents enables us to derive the entropy production rate and the attractor dimension. In chaotic systems, information flows from microstates to macrostates due to the sensitivity to initial conditions [70]. This creation of information about initial conditions is captured by the Kolmogorov-Sinai entropy, also called metric entropy or just entropy production rate. An upper bound to the Kolmogorov-Sinai entropy $H_{\mathrm{KS}} \leq H$ is given by the sum of the positive Lyapunov exponents [71]:

$$
H=\sum_{\lambda_{i}>0} \lambda_{i}
$$

For smooth densities $\rho$ of the physical measure along the unstable directions the Pesin identity $H_{\mathrm{KS}}=H$ holds. The validity of the Pesin identity is equivalent with $\rho$ being a Sinai-Bowen-Ruelle (SRB) measure [72]. Keeping this in mind, we will call $H$ the entropy production rate throughout this thesis.

The ordered Lyapunov exponents additionally yield the Kaplan-Yorke dimension, also known as Lyapunov dimension via

$$
D=d+\frac{\sum_{i=1}^{d} \lambda_{i}}{\left|\lambda_{d+1}\right|} \quad d=\max \left\{n: \sum_{i=1}^{n} \lambda_{i} \geq 0\right\} .
$$

This is an upper bound to the information dimension $D_{1} \leq D$ [73], where the equality was originally conjectured by Kaplan and Yorke for SRB measures [74]. If the Kaplan-Yorke conjecture 
holds, then $\rho$ is an SRB measure and thus the Pesin identity holds as well [72]. Keeping this in mind, we will call $D$ the attractor dimension throughout this thesis. These fundamental quantities, derived from the Lyapunov spectra, are topological invariants of the dynamical system and play the central role in our analysis of the dynamics of neural networks.

For an intuition of Eq. (1.5) and (1.6), consider that each positive Lyapunov exponent stands for the exponential rate of separation of initial conditions in one direction. This leads to distinguishable macroscopic states, thus producing entropy. The sum of all positive Lyapunov exponents yields the overall rate of entropy production, Eq. (1.5). Each negative Lyapunov exponent stands for the exponential rate of contraction of initial conditions in one direction. The number of directions $d$ in Eq. (1.6) symbolizes a balance of separation and contraction, as it counts the number of ordered Lyapunov exponents for which the sum remains nonnegative. It can be seen as an estimate of the dimensionality of a hypersphere representing possible perturbations along the trajectory, whose volume is preserved. The second term in Eq. (1.6) is a linear interpolation for the general case where the $\operatorname{sum} \sum_{i=1}^{d} \lambda_{i}$ is not exactly equal to zero.

The relation to different definitions of entropies and dimensions is best seen using the Rényi entropy formalism (more details can be found in, e.g., [67,75]). Assuming a finite partition with length scale $\alpha$ of the support of the physical measure with the probability distribution $p_{i}$ assigned to each partition $i$, the Rényi entropies of order $q$ are

$$
H_{q}=\frac{1}{1-q} \log _{2} \sum_{i=1}^{N_{\alpha}} p_{i}^{q},
$$

where $q>0, q \neq 1$ and the total number of non-empty partitions with length scale $\alpha$ is denoted $N_{\alpha}$. The order-0 Rényi entropy recovers the Hartley entropy $H_{0}=\log _{2} N_{\alpha}$ and the limit $q \rightarrow 1$, using L'Hôpital's rule, yields the Shannon entropy $H_{1}=-\sum_{i} p_{i} \log _{2} p_{i}$. With the Rényi entropy definition (1.7) and length scale $\alpha$, one can generalize the definition of dimensions [75]:

$$
D_{q}=\lim _{\alpha \rightarrow 0} \frac{H_{q}}{\log _{2}(1 / \alpha)}
$$

Then, $q=0$ represents the attractor dimension (a.k.a. capacity, box-counting or Hausdorff dimension): $D_{0}=\lim _{\alpha \rightarrow 0} \frac{\log _{2} N_{\alpha}}{\log _{2}(1 / \alpha)}, q \rightarrow 1$ represents the information dimension and $q=2$ the correlation dimension (the spatial correlation between the distance of points on the attractor). Generally, for any $q_{1}<q_{2}$, one gets $D_{q_{1}} \geq D_{q_{2}}$ and in the case of true inequality one speaks of multifractality of the physical measure.

The entropy production rate $H$ quantifies the uncertainty in predicting the future, conditioned on knowing the present. In a stable deterministic system, knowledge of the present state fully with a certain precision determines all future states, and there is no uncertainty left. This is displayed in Fig. 1.5A. The present state at $t=0$ is identified as a square in the symbolic phase space. In this stable system, small differences in the initial conditions decay exponentially over time, characterized by a negative Lyapunov exponent $\lambda^{-}<0$. Thus, future states are fully predictable. In a chaotic system, this is different (Fig. 1.5B). Future states still remain uncertain when the present state is known with a certain precision, due to the sensitivity to initial conditions. Besides a stable direction, there is also an unstable direction in which small perturbations increase exponentially at a rate $\lambda^{+}>0$. After some time, a microscopic perturbation below the precision leads to a different macroscopic future state. Because the initial conditions cannot be known with indefinite precision, the future is not fully predictable. The uncertainty left in a future state is given by the 

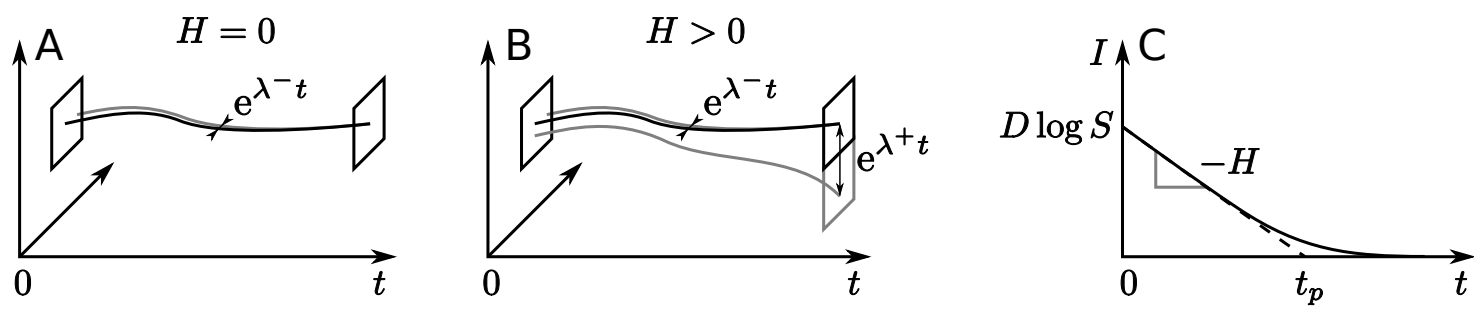

Figure 1.5 - Information loss in chaotic systems. (A) Stable system: future states are fully predictable after measuring the present state (states correspond to squares in the symbolic phase space). (B) Chaotic system: future states remain uncertain due to the sensitivity to initial conditions. (C) The mutual information between future and present decreases over time at the entropy production rate $H$. The maximal information in one measurement is $I_{0} \sim D \log S$ where $D$ is the attractor dimension and $S$ is the signal to noise ratio or precision of the measurement. The maximal predictive time can be estimated to $t_{p} \approx I_{0} / H=D \log S / H$.

entropy production rate $H$, Eq. (1.5), as the sum of all positive Lyapunov exponents. Measuring the future state then yields additional information about the initial conditions of the present state. This additional information in the macrostate about the microstate is created by the chaotic dynamics and captured in the entropy production rate.

Knowledge of the attractor dimension $D$, Eq. (1.6), allows us to estimate the maximal information gained in one measurement. The entropy production rate $H$, Eq. (1.5), allows us to estimate the rate of entropy production in subsequent measurements. Since they are related quantities, one should discuss them together [76, 77]. Suppose that the information in the network is encoded in the networks' states with a certain precision $\alpha$. This can also be thought of as the inverse of a signal-to-noise ratio $S$. The information theoretic capacity, the upper bound of information gained by one measurement of the network state, is then given by $I_{0} \sim D \log S$. The information about the present state decreases over time, as it is overwritten by the entropy produced at rate $H$. The mutual information between the future and present state therefore decreases proportional to the entropy production rate $\left(I=I_{0}-H t\right.$, Fig. $\left.1.5 \mathrm{C}\right)$. The actual time after which the initial information is lost due to the chaotic dynamics depends on the amount of the initial information and the entropy production rate, but even the maximal input information is lost after the typical predictive time $t_{p} \approx I_{0} / H=D \log S / H \sim D / H$.

This demonstrates that the attractor dimension $D$ and entropy production rate $H$ derived from the Lyapunov spectra of models of cortical circuits provide valuable insight for the understanding of information processing in cortical networks. Accurately calculating the full Lyapunov spectra of large spiking neuron networks is thus of fundamental importance for the characterization of information preservation and decay in models of cortical circuits. In the following chapter, we present a general approach only requiring the precise knowledge of the neurons' spike times and their phase-response curves (a.k.a. phase-resetting curves), applicable to networks with arbitrary topologies. We also derive a random matrix approximation of the mean Lyapunov exponent. This approach permits a comprehensive and precise analysis of the dynamics of neural networks.

\subsection{Weak Pairwise Correlations in Strongly Correlated Network States}

We have mentioned in Section 1.1 that the balanced state is characterized by weak pairwise spike correlations in sparse and even densely coupled network [3,32]. Weak pairwise spike correlations 


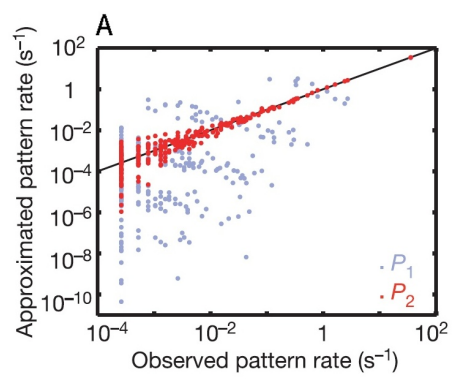

B

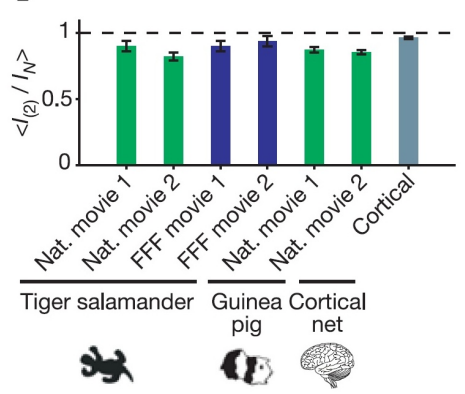

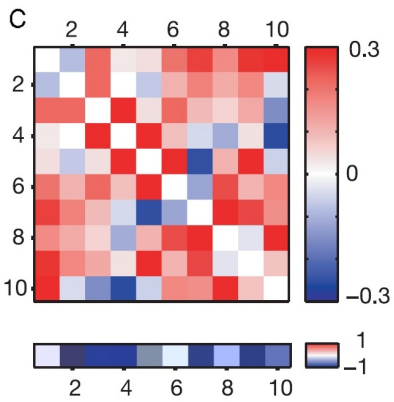

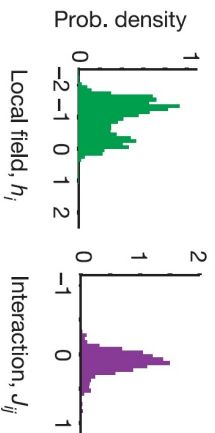

Figure 1.6 - Weak pairwise correlations imply strongly correlated network states. (Figures from Ref. [78]) (A) The distribution of observed 10-cell patterns in the spike trains of retinal ganglion cells in response to a natural movie does not agree with the result of assuming independence of the cells $\left(P_{1}\right)$. The maximum entropy model fitted with the pairwise correlations of the cells largely resolves this discrepancy $\left(P_{2}\right)$. (B) $90 \%$ of the multi-information in such cell patterns in different species and cortical cultures can be explained by the maximum entropy model. (C) Pairwise interactions $J_{i j}$ between the cells (matrix) and the local field at each cell $h_{i}$ (row) in the maximum entropy model, the distributions are displayed on the right.

can easily be mistaken to mirror the absence of higher order correlations. This is not the case [78].

Weak pairwise correlations imply that higher order correlations lead to the collective behavior observed in populations of neurons. This was demonstrated by the success of maximum entropy models in predicting quite accurately the collective behavior of neural populations in the retina [78,79] and in cortical networks in vitro [78, 80] and in vivo [81].

We would like to recall the argumentation for the success of the maximum entropy model introduced in Ref. [78] (Fig. 1.6). It was observed that weak pairwise correlations coexist with a collective behavior indicated by a strong discrepancy between the distribution of observed firing patterns of 10 cells in a retinal patch with the results obtained from assuming the cells to be independent (Fig. 1.6A). The discrepancy was largely resolved when comparing the true distribution with the one obtained from the prediction of the maximum entropy model. Interestingly, for the maximum entropy model it is sufficient to use the measured pairwise spike correlations which are weak but widespread throughout the population (Fig. 1.6C). These weak pairwise correlations are used to model the strengths of pairwise interactions in an Ising model which is the exact mapping for a maximum entropy model consistent with at most pairwise correlations. Considering only pairwise interactions does not mean that higher order correlations are suppressed. In fact, this minimally structured model was able to predict about $90 \%$ of the multi-information in the real population (Fig. 1.6B). Therefore, the authors conclude that "although the pairwise correlations are small and the multi-neuron deviations from independence are large, the maximum entropy model consistent with the pairwise correlations captures almost all of the structure in the distribution of responses from the full population of neurons. Thus the weak pairwise correlations imply strongly correlated states." [78]

A recent study has confirmed that higher order correlations are not weak [82], despite the success of the maximum entropy model fitted with only the weak pairwise correlations. The authors developed a perturbation theory of the entropy of Ising systems up to fourth order and reanalyzed the data from Ref. [78]. They show that "even though all pairwise correlations are weak, the fact that the correlations are widespread means that their impact on the network as a whole is not captured in the leading orders of perturbation theory." [78] This means that the success of maximum 
entropy models lies in the fact that the pairwise interactions considered in this model are sufficient to capture higher order correlations, but not that higher order correlations are negligible. In their study there is no sign of saturation for increasing order of the perturbation theory. A relevant question is therefore how many orders of higher correlations must be taken into account in realistic neural networks. We believe that the calculation of the attractor dimension $D$ with our approach helps to answer this question. This will be discussed in Section 3.6. 


\section{Networks of Theta Neurons The Standard Model of Type I Membranes}

In this chapter, we will develop the general approach for the characterization and quantification of spiking neuron networks. This approach can be applied to arbitrary network topologies and single neuron phase-response curves. As a typical model of neural networks we will study random networks of theta neurons in the balanced state. The balanced state is the prevailing explanation of the asynchronous irregular firing activity of cortical networks [2, 3, 12, 13]. The theta neuron model is the standard form of type I excitable neurons [83-85]. Hence, it is expected that the derived results are representative of a wide class of neural network models.

The characterization and quantification of spiking neuron networks developed here is based on the numerically exact calculation of the complete spectra of Lyapunov exponents. For highdimensional dynamical systems such as neural networks, this requires highly precise methods. Our approach is based on exact closed-form expressions for the time evolution of all neurons' states. These form a map that iterates the neurons' states from one spike event in the networks to the next. This map is evaluated iteratively in event-based simulations following Ref. [51,54, 58]. Its derivative called the single spike Jacobian is also obtained analytically, and builds the foundation for the numerical computation of the Lyapunov spectra in the standard Gram-Schmidt reorthonormalization procedure described in Ref. [68, 69].

In the first section, the general class of models is described to which the approach for the calculation of the Lyapunov spectra can be straightforwardly applied. This is followed by the derivation of the iterative map and the single spike Jacobian in a general form and for arbitrary phase-response curves in particular. Then, we discuss the neuron model-independent characteristics of the balanced state in inhibitory and excitatory-inhibitory networks. A specific parametrization of the coupling strengths in the excitatory-inhibitory networks preserves the statistics of the inhibitory networks, enabling a quantitative comparison of the two types of networks.

In Section 2.5, the introduced approach is then applied to sparse networks of theta neurons, completed with a derivation of the mean Lyapunov exponent in a random matrix approximation and a discussion of the numerical procedure and the convergence of the Lyapunov spectra.

After the introduction of the general approach and the methodological details of theta neuron networks, we present a comprehensive analysis of theta neuron networks starting in Section 2.6 . This includes the discussion of two phase transitions from the asynchronous state to synchronous states and most importantly the analysis of the dynamics of inhibitory and excitatory-inhibitory networks based on their Lyapunov spectra. The chapter ends with an analysis of the Lyapunov vectors and an analogy of the observed type of chaos with spatiotemporal chaos. Some of these results are published in letter form in Ref. [8]. 


\subsection{General Neural Network Model}

We consider pulse-coupled neural networks without synaptic delays. The neurons are arranged on directed graphs, such that each neuron has a defined set of pre- and postsynaptic neurons. At each spike time of a neuron, a current pulse is immediately received by its postsynaptic neurons, changing their states instantaneously. These networks of $N$ coupled neurons are described by a system of differential equations of the neurons' membrane potentials (voltages) $V_{i}$, with $i=1 \ldots N$ :

$$
\tau_{\mathrm{m}} \frac{\mathrm{d} V_{i}(t)}{\mathrm{d} t}=F\left(V_{i}(t)\right)+I_{i}(t)
$$

The integration time constant (or membrane time constant) is denoted $\tau_{\mathrm{m}}$. The single neuron dynamics are described by the function $F\left(V_{i}\right)$ and the connections between the neurons are captured in the synaptic input current $I_{i}(t)$. The synaptic input currents to the neurons are:

$$
I_{i}(t)=I_{T}+I_{i}^{\mathrm{ext}}+\sum_{j \in \operatorname{pre}(i)} \sum_{s} J_{i j} \tau_{\mathrm{m}} \delta\left(t-t_{j}^{(s)}\right) .
$$

Without loss of generality but for an easy comparison of different neuron models, the synaptic input currents compensate an intrinsic rheobase current $I_{T}$ of the individual neuron model. Then the second term, the constant external excitatory current $I_{i}^{\text {ext }}>0$ is always a suprathreshold input current. The compensation of the rheobase current in Eq. (2.2) is not crucial, it can also be set to zero if wanted. The only difference then is that the external current $I_{i}^{\text {ext }}$ must be larger than $I_{T}$ for tonic firing and this value depends on the considered neuron model.

The important last term in the synaptic input currents (2.2) describes the connections in the network. The neurons receive nondelayed $\delta$-pulses of strength $J_{i j}$ at the spike times $\left\{t_{j}^{(s)}\right\}$ of their presynaptic neurons $j \in \operatorname{pre}(i)$. This induces a step-like change in the spike-receiving neuron's voltage $V_{i}\left(t_{j}^{(s)}+\varepsilon\right)=V_{i}\left(t_{j}^{(s)}-\varepsilon\right)+J_{i j}$ for $\varepsilon \rightarrow 0$.

This formalism allows the connectivity between neurons to be chosen arbitrarily. Furthermore, single neuron properties such as external currents and synaptic coupling strengths can differ between neurons without restrictions, allowing for heterogeneous networks.

We are focusing on nondelayed synaptic couplings, because the networks' phase space is then of fixed and finite dimensionality. This has the advantage of well-defined Lyapunov spectra. Including synaptic delays would increase the networks' phase spaces at best to a varying dimensionality [86]. Consequently, the calculation of the complete Lyapunov spectra as proposed here would become difficult, and is for now avoided by considering nondelayed synaptic couplings.

\subsection{Iterative Map}

For the general class of neural network models, we now introduce the map that iterates all neurons' states from one spike time in the network to the next. The neurons' states are described by their voltages or phases, denoted $x_{i}$ here. Successive spikes in the whole network are denoted $\left\{t_{s}\right\}$. The iterative map that iterates the neurons' states in the interval $\left(t_{s}, t_{s+1}\right]$ consists of two parts described by the functions $f$ and $g$. First the states of all neurons $i=1, \ldots, N$ are evolved with

$$
f\left(x_{i}\left(t_{s}\right), t_{s+1}-t_{s}\right)=x_{i}\left(t_{s+1}^{-}\right)
$$


from the time $t_{s}$ just after the last spike to the time $t_{s+1}^{-}$just before the spike considered in this iteration. The spiking neuron in this iteration is denoted $j^{*}$. For the neurons $i \notin \operatorname{post}\left(j^{*}\right)$ that do not receive a spike in this interval we take $x_{i}\left(t_{s+1}^{-}\right)=x_{i}\left(t_{s+1}\right)$. The neurons $i \in \operatorname{post}\left(j^{*}\right)$ that receive this spike are then updated with the function

$$
g\left(x_{i}\left(t_{s+1}^{-}\right)\right)=x_{i}\left(t_{s+1}\right) .
$$

The spiking neuron is reset from the threshold $x_{T}$ to the reset value $x_{R}$. The functions $f$ and $g$ are derived from the governing equation (2.1) with Eq. (2.2) and the facts that between spikes the neurons only receive constant external currents and receiving a spike results in an instantaneous change in the neurons' voltages.

The composition of $f$ and $g$ thus yields the map that iterates the neurons' states

$$
x_{i}\left(t_{s+1}\right)= \begin{cases}f\left(x_{i}\left(t_{s}\right), t_{s+1}-t_{s}\right) & \text { if } i \notin \operatorname{post}\left(j^{*}\right) \\ g\left(f\left(x_{i}\left(t_{s}\right), t_{s+1}-t_{s}\right)\right) & \text { if } i \in \operatorname{post}\left(j^{*}\right) .\end{cases}
$$

For true event-based simulations an exact expression of the next spike time is also necessary to obtain. The next spike time $t_{s+1}$ is implicitly given from the evolution function $f$ between spikes, since for the spiking neuron $j^{*}$ holds $f\left(x_{j^{*}}\left(t_{s}\right), t_{s+1}-t_{s}\right)=x_{\mathrm{T}}$ with $x_{\mathrm{T}}$ denoting the threshold to spike. If $f$ is invertible with respect to time, this leads directly to an exact expression of the next spike time in the network:

$$
t_{s+1}=t_{s}+\min _{i}\left\{f^{-1}\left(x_{\mathrm{T}}-x_{i}\left(t_{s}\right)\right)\right\} .
$$

The neuron $j^{*}$ with the minimal time to its next spike $f^{-1}\left(x_{\mathrm{T}}-x_{i}\left(t_{s}\right)\right)$ will fire next in the network at time $t_{s+1}$. The exact expressions (2.3) and (2.4) are the ingredients for fast and precise eventbased simulations. The event-based simulation is then iterating the procedure of first calculating the next spike time with Eq. (2.4) and second evolving all neurons' states with the map (2.3) and resetting the spiking neuron from $x_{T}$ to $x_{R}$.

\subsection{Single Spike Jacobian}

Essential for our approach is the derivation of the derivative of the map (2.3), called the single spike Jacobian. It describes the linear evolution of infinitesimal perturbations of the neurons' states and will be used to numerically calculate the Lyapunov spectra. We will first derive the single spike Jacobian for an arbitrary map (2.3) composed of the function $f$ and $g$. The function $f$ descsribes the evolution of the neurons' states between two spike events when only the constant external currents are received and function $g$ describes the updates of the neurons' states when they receive a spike of a presynaptic neuron.

Since infinitesimal perturbations are considered here, the spike-order in the networks is preserved. This is true as long as there are no exactly synchronous spike events which generally should not occur in the considered asynchronous networks. If the perturbation of the spiking neuron was finite, its spike time could change in the considered interval. For illustration and clarity, we will therefore shift the time interval of the map (2.3) by a fixed amount $\delta$ in the derivation of the single spike Jacobian. This is to guarantee that the possibly altered spike time, denoted $\tau_{s+1}$, remains in the considered interval of the map. With a suitably large time shift $\delta$ the map thus evolves all states from $t_{s} \mapsto t_{s}+\delta$ through $t_{s+1} \mapsto t_{s+1}+\delta$. The only difference is that with this shift the 
spike in the considered interval does not occur exactly at the end of the interval $t_{s+1}$ as before but within the interval at $\tau_{s+1}<t_{s+1}$. Because the actually considered perturbations are infinitesimally small, the actual spike time of neuron $j^{*}$ cannot change and at the end of the derivation of the Jacobian the limit $\delta \rightarrow 0$ will be taken and $t_{s+1}$ identified with $\tau_{s+1}$.

The map with the shifted but fixed time interval $\left(t_{s}, t_{s+1}\right]$ and the spike of neuron $j^{*}$ occurring within this interval at time $\tau_{s+1}$ reads:

$$
x_{i}\left(t_{s+1}\right)= \begin{cases}f\left(x_{i}\left(t_{s}\right), t_{s+1}-t_{s}\right) & \text { if } i \notin \operatorname{post}\left(j^{*}\right) \\ f\left(g\left(f\left(x_{i}\left(t_{s}\right), \tau_{s+1}-t_{s}\right)\right), t_{s+1}-\tau_{s+1}\right) & \text { if } i \in \operatorname{post}\left(j^{*}\right) .\end{cases}
$$

The important derivative of the map, the single spike Jacobian matrix is:

$$
\mathbf{D}\left(t_{s}\right)=\frac{\mathrm{d} \vec{x}\left(t_{s+1}\right)}{\mathrm{d} \vec{x}\left(t_{s}\right)}
$$

The Jacobian elements of the neurons $i^{0} \notin \operatorname{post}\left(j^{*}\right)$ that do not receive the spike of the spiking neuron $j^{*}$ in the considered interval are:

$$
\begin{aligned}
D_{i^{0}, j}\left(t_{s}\right) & =\frac{\mathrm{d} x_{i^{0}}\left(t_{s+1}\right)}{\mathrm{d} x_{j}\left(t_{s}\right)} \\
& =\partial_{x} f\left(x_{i^{0}}\left(t_{s}\right), t_{s+1}-t_{s}\right) \delta_{i^{0} j}
\end{aligned}
$$

where $\partial_{x} f=\frac{\partial f(x, t)}{\partial x}$ denotes the derivative of $f$ with respect to the state $x$, and $\delta_{i^{0} j}$ is the Kronecker delta. Thus, all but the diagonal elements vanish in the part of the Jacobian corresponding to the neurons that do not receive the spike in the considered interval.

The diagonal Jacobian elements of the spike receiving neurons $i^{*} \in \operatorname{post}\left(j^{*}\right)$ are also straightforwardly derived using the chain rule:

$$
\begin{aligned}
D_{i^{*}, i^{*}}\left(t_{s}\right) & =\frac{\mathrm{d} x_{i^{*}}\left(t_{s+1}\right)}{\mathrm{d} x_{i^{*}}\left(t_{s}\right)} \\
& =\partial_{x} f\left(x_{i^{*}}\left(\tau_{s+1}^{+}\right), t_{s+1}-\tau_{s+1}\right) \partial_{x} g\left(x_{i^{*}}\left(\tau_{s+1}^{-}\right)\right) \partial_{x} f\left(x_{i^{*}}\left(t_{s}\right), \tau_{s+1}-t_{s}\right)
\end{aligned}
$$

where we denoted the state just before spike reception by $x_{i^{*}}\left(\tau_{s+1}^{-}\right)=f\left(x_{i^{*}}\left(t_{s}\right), \tau_{s+1}-t_{s}\right)$ and the state just after spike reception by $x_{i^{*}}\left(\tau_{s+1}^{+}\right)=g\left(x_{i^{*}}\left(\tau_{s+1}^{-}\right)\right)$.

There are also nondiagonal elements since the spike receiving neurons are influenced by the possibly changed spike time $\tau_{s+1}$ that results from a perturbation of the spiking neuron. All other nondiagonal elements vanish, see Eq. (2.7). The nonzero nondiagonal Jacobian elements are:

$$
\begin{aligned}
D_{i^{*}, j^{*}}\left(t_{s}\right)= & \frac{\mathrm{d} x_{i^{*}}\left(t_{s+1}\right)}{\mathrm{d} x_{j^{*}}\left(t_{s}\right)} \\
= & \partial_{x} f\left(x_{i^{*}}\left(\tau_{s+1}^{+}\right), t_{s+1}-\tau_{s+1}\right) \partial_{x} g\left(x_{i^{*}}\left(\tau_{s+1}^{-}\right)\right) \partial_{t} f\left(x_{i^{*}}\left(t_{s}\right), \tau_{s+1}-t_{s}\right) \frac{\partial \tau_{s+1}}{\partial x_{j^{*}}\left(t_{s}\right)}- \\
& \partial_{t} f\left(x_{i^{*}}\left(\tau_{s+1}^{+}\right), t_{s+1}-\tau_{s+1}\right) \frac{\partial \tau_{s+1}}{\partial x_{j^{*}}\left(t_{s}\right)},
\end{aligned}
$$


with the partial derivative $\partial_{t} f=\frac{\partial f(x, t)}{\partial t}$.

The dependence of the spike time on the spiking neuron's state $\frac{\partial \tau_{s+1}}{\partial x_{j^{*}}\left(t_{s}\right)}$ might be obtained directly from the inverse of function $f$, Eq. (2.4). For the derivation of the Jacobian, it is however not required that $f$ is invertible, since the necessary derivative can also be obtained from the implicit definition of the next spike time $f\left(x_{j^{*}}\left(t_{s}\right), \tau_{s+1}-t_{s}\right)=x_{\mathrm{T}}$. Thus, other numerically precise methods to find the next spike time can be used in the network simulations, while the single spike Jacobian can still be evaluated exactly as long as $f$ and $g$ are differentiable. From the implicit definition of the next spike time, one gets

$$
\begin{aligned}
\frac{\mathrm{d} f\left(x_{j^{*}}\left(t_{s}\right), \tau_{s+1}-t_{s}\right)}{\mathrm{d} \tau_{s+1}}=0 & =\partial_{x} f\left(x_{j^{*}}\left(t_{s}\right), \tau_{s+1}-t_{s}\right) \frac{\partial x_{j^{*}}\left(t_{s}\right)}{\partial \tau_{s+1}}+\partial_{t} f\left(x_{j^{*}}\left(t_{s}\right), \tau_{s+1}-t_{s}\right) \\
\frac{\partial \tau_{s+1}}{\partial x_{j^{*}}\left(t_{s}\right)} & =-\frac{\partial_{x} f\left(x_{j^{*}}\left(t_{s}\right), \tau_{s+1}-t_{s}\right)}{\partial_{t} f\left(x_{j^{*}}\left(t_{s}\right), \tau_{s+1}-t_{s}\right)} .
\end{aligned}
$$

Inserting this in Eq. (2.9) yields the nondiagonal elements

$$
\begin{aligned}
D_{i^{*}, j^{*}}\left(t_{s}\right)= & \partial_{x} f\left(x_{j^{*}}\left(t_{s}\right), \tau_{s+1}-t_{s}\right)\left(\frac{\partial_{t} f\left(x_{i^{*}}\left(\tau_{s+1}^{+}\right), t_{s+1}-\tau_{s+1}\right)}{\partial_{t} f\left(x_{j^{*}}\left(t_{s}\right), \tau_{s+1}-t_{s}\right)}-\right. \\
& \left.\partial_{x} f\left(x_{i^{*}}\left(\tau_{s+1}^{+}\right), t_{s+1}-\tau_{s+1}\right) \partial_{x} g\left(x_{i^{*}}\left(\tau_{s+1}^{-}\right)\right) \frac{\partial_{t} f\left(x_{i^{*}}\left(t_{s}\right), \tau_{s+1}-t_{s}\right)}{\partial_{t} f\left(x_{j^{*}}\left(t_{s}\right), \tau_{s+1}-t_{s}\right)}\right) .
\end{aligned}
$$

This equation can also be used for the last remaining term, the diagonal element corresponding to the spiking neuron $j^{*}$. The spiking neuron is reset from $x_{T}$ to $x_{R}$ at its spike time $\tau_{s+1}$, thus $g\left(x_{j^{*}}\left(\tau_{s+1}^{-}\right)\right)=x_{R}$ and therefore $\partial_{x} g\left(x_{j^{*}}\left(\tau_{s+1}^{-}\right)\right)=0$. This leads to the following diagonal element of the spiking neuron $j^{*}$ :

$$
D_{j^{*}, j^{*}}\left(t_{s}\right)=\partial_{x} f\left(x_{j^{*}}\left(t_{s}\right), \tau_{s+1}-t_{s}\right) \frac{\partial_{t} f\left(x_{j^{*}}\left(\tau_{s+1}^{+}\right), t_{s+1}-\tau_{s+1}\right)}{\partial_{t} f\left(x_{j^{*}}\left(t_{s}\right), \tau_{s+1}-t_{s}\right)}
$$

Equations (2.7), 2.8), 2.11) and 2.12) constitute the single spike Jacobians for arbitrary functions $f$ and $g$, where $f$ determines the time evolution for constant input currents between spike events and $g$ determines the update of the states when a spike is received. Taking the limit of infinitesimal perturbations, thus $\tau_{s+1}^{+} \rightarrow t_{s+1}$ we arrive at:

$$
\begin{aligned}
D_{i j}\left(t_{s}\right)= & \partial_{x} f\left(x_{j}\left(t_{s}\right), t_{s+1}-t_{s}\right)\left(\delta_{i j} \delta_{i i^{0}}+\frac{\partial_{t} f\left(x_{j^{*}}\left(t_{s+1}\right), 0^{+}\right)}{\partial_{t} f\left(x_{j^{*}}\left(t_{s}\right), t_{s+1}-t_{s}\right)} \delta_{i j} \delta_{i j^{*}}+\right. \\
& \partial_{x} f\left(x_{i^{*}}\left(t_{s+1}\right), 0^{+}\right) \partial_{x} g\left(x_{i^{*}}\left(t_{s+1}^{-}\right)\right) \delta_{i j} \delta_{i i^{*}}+\frac{\partial_{t} f\left(x_{i^{*}}\left(t_{s+1}\right), 0^{+}\right)}{\partial_{t} f\left(x_{j^{*}}\left(t_{s}\right), t_{s+1}-t_{s}\right)} \delta_{i i^{*}} \delta_{j j^{*}}- \\
& \left.\partial_{x} f\left(x_{i^{*}}\left(t_{s+1}\right), 0^{+}\right) \partial_{x} g\left(x_{i^{*}}\left(t_{s+1}^{-}\right)\right) \frac{\partial_{t} f\left(x_{i^{*}}\left(t_{s}\right), t_{s+1}-t_{s}\right)}{\partial_{t} f\left(x_{j^{*}}\left(t_{s}\right), t_{s+1}-t_{s}\right)} \delta_{i i^{*}} \delta_{j j^{*}}\right)
\end{aligned}
$$


In matrix form the single spike Jacobian reads

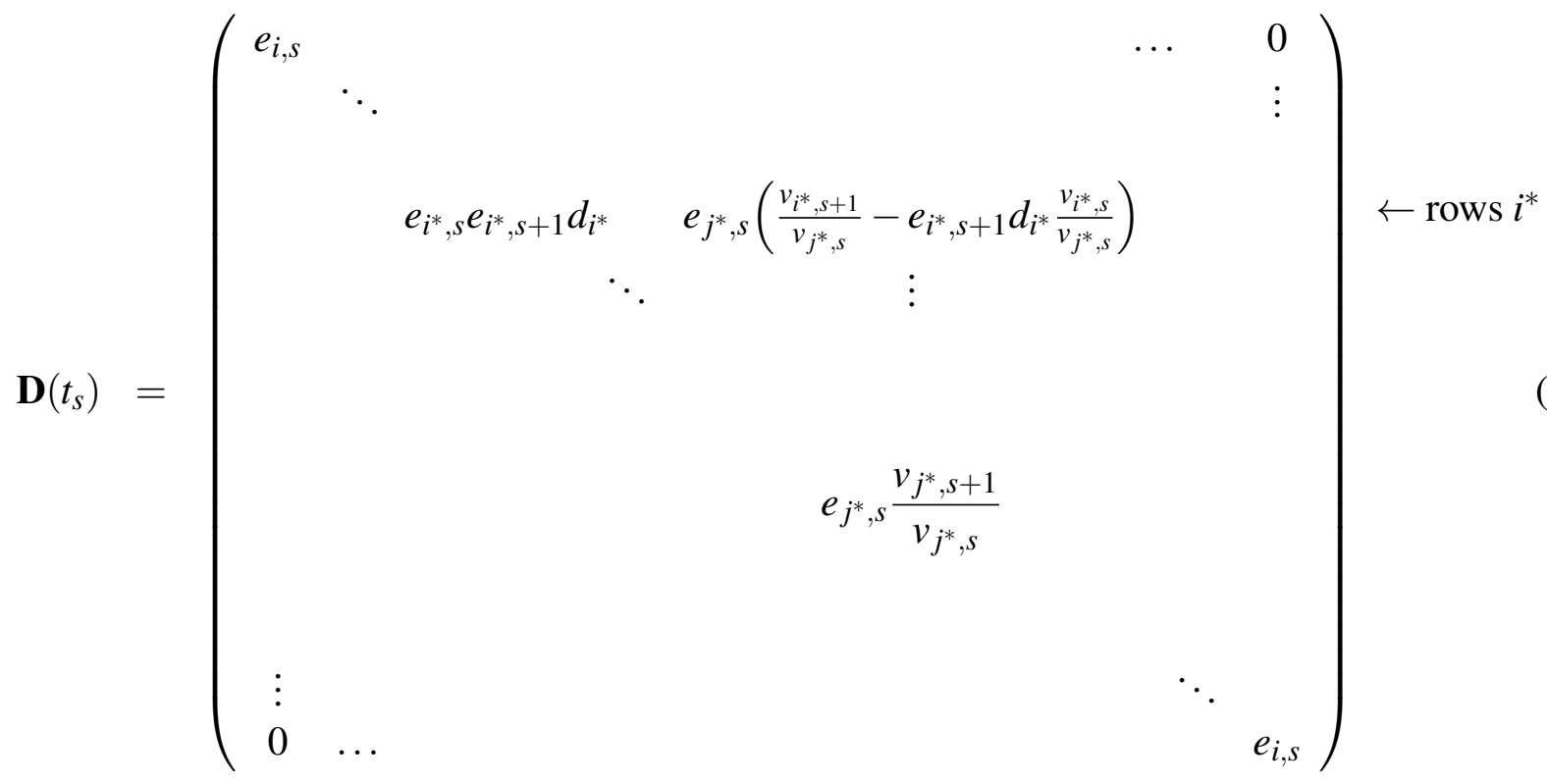

$$
\begin{aligned}
& \text { column } j^{*}
\end{aligned}
$$

with the abbreviations

$$
\begin{aligned}
e_{i, s} & =\partial_{x} f\left(x_{i}\left(t_{s}\right), t_{s+1}-t_{s}\right) & e_{i, s+1} & =\partial_{x} f\left(x_{i}\left(t_{s+1}\right), 0^{+}\right) \\
d_{i} & =\partial_{x} g\left(x_{i}\left(t_{s+1}^{-}\right)\right) & & \\
v_{i, s} & =\partial_{t} f\left(x_{i}\left(t_{s}\right), t_{s+1}-t_{s}\right) & v_{i, s+1} & =\partial_{t} f\left(x_{i}\left(t_{s+1}\right), 0^{+}\right) .
\end{aligned}
$$

The single spike Jacobians (2.13) are composed of three different parts. One part constitutes the diagonal elements $e_{i, s}$ related to the neurons that are not postsynaptic to the spiking neuron. These neurons only contribute diagonal elements since only perturbations of their own states have an effect. Then, there are the elements related to the postsynaptic neurons $i^{*} \in \operatorname{post}\left(j^{*}\right)$ of the spiking neuron $j^{*}$. Their diagonal elements $e_{i^{*}, s} e_{i^{*}, s+1} d_{i^{*}}$ also reflect the consequence of perturbations of their own state. The nonzero nondiagonal elements $e_{j^{*}, s}\left(\frac{v_{i^{*}, s+1}}{v_{j^{*}, s}}-e_{i^{*}, s+1} d_{i^{*}} \frac{v_{i^{*}, s}}{v_{j^{*}, s}}\right)$ reflect the influence of a perturbation of the spiking neuron. If the spiking neuron is perturbed, its spike time changes and the postsynaptic neurons receive the current pulse at a different time. This leads to a different state of the postsynaptic neurons that is seen as an effective perturbation.

\subsubsection{Single Spike Jacobian from Phase Response Curves}

Importantly, the single spike Jacobians can be conveniently expressed using the single neuron phase-response curves. In the phase description, neurons are described by their state relative to an equivalent, uncoupled, free-running neuron. We denote this phase $\phi_{i}$. The phase-response curve $Z\left(\phi_{i}\right)$, also called phase-resetting curve, quantifies the change of a neurons phase in response to a perturbation received at a certain phase (see, e.g., [87]).

Assuming a constant phase velocity $\omega_{i}$ between spikes the functions $f$ and $g$ are simply $f\left(\phi_{i}, t\right)=$ 
$\phi_{i}+\omega_{i} t$ and $g\left(\phi_{i}\right)=\phi_{i}+Z\left(\phi_{i}\right)$. Thus the iterative map, Eq. (2.3), reads

$$
\phi_{i}\left(t_{s+1}\right)=\phi_{i}\left(t_{s}\right)+\omega_{i}\left(t_{s+1}-t_{s}\right)+Z\left(\phi_{i}\left(t_{s}\right)+\omega_{i}\left(t_{s+1}-t_{s}\right)\right) \delta_{i \in \operatorname{post}\left(j^{*}\right)},
$$

where $\delta_{i \in \operatorname{post}\left(j^{*}\right)}$ is one if $i$ is a postsynaptic neuron of the spiking neuron $j^{*}$ and zero otherwise.

The derivatives are $e_{i}=\partial_{\phi} f=1, v_{i}=\partial_{t} f=\omega_{i}$ and $d_{i}=\partial_{\phi} g=1+Z^{\prime}\left(\phi_{i}\right)$, where $Z^{\prime}\left(\phi_{i}\right)$ denotes the derivative of the phase-response curve with respect to the phase. Thus, the single spike Jacobian (2.13) reads

$$
D_{i j}\left(t_{s}\right)= \begin{cases}1+Z^{\prime}\left(\phi_{i^{*}}\left(t_{s+1}^{-}\right)\right) & \text {for } i=j=i^{*} \\ -\frac{\omega_{i^{*}}}{\omega_{j^{*}}} Z^{\prime}\left(\phi_{i^{*}}\left(t_{s+1}^{-}\right)\right) & \text {for } i=i^{*} \text { and } j=j^{*} \\ \delta_{i j} & \text { otherwise, }\end{cases}
$$

where $j^{*}$ denotes the spiking neuron in the considered interval, firing at time $t_{s+1}, i^{*} \in \operatorname{post}\left(j^{*}\right)$ are the spike receiving neurons and $\delta_{i j}$ is the Kronecker delta. The derivatives of the phaseresponse curves $Z^{\prime}(\phi)$ are evaluated at the phases of the spike receiving neurons $\phi_{i^{*}}\left(t_{s+1}^{-}\right)=$ $\phi_{i^{*}}\left(t_{s}\right)+\omega_{i^{*}}\left(t_{s+1}-t_{s}\right)$ just before spike reception.

In cases when the single neuron model cannot be solved analytically, the expression of the single spike Jacobian in terms of the phase-response curves 2.15 can still be used to calculate the Lyapunov spectrum. An example of a one-dimensional single neuron model is the exponential integrate and fire model [88], whose phase-response curve can be calculated numerically. This needs to be done only once. Then, either a numerical fit or a look-up table of the derivative of the phaseresponse curve can be used for the single spike Jacobians (2.15). The network simulations should be performed with high-precision algorithms, leading to the necessary spike times and neurons' phases. With this procedure, the Lyapunov spectra can be obtained with very high precision despite the lack of an exact derivation of the phase-response curve. In the case of higher-dimensional single neuron models, the phase-response curves and network simulations could also be obtained with high-precision integration schemes. There is however an additional approximation whose validity should be addressed. In the calculation of the Lyapunov spectra as proposed here, one would use an approximated one-dimensional phase description with the numerically obtained phase-response curve. Since this does not capture the full dynamics precisely, one needs to consider how crucial this approximation would be for higher dimensional neuron models.

\subsubsection{From Single Spike Jacobians to Lyapunov exponents}

The product of the single spike Jacobians 2.13 asymptotically leads to the Lyapunov exponents (see Section 1.4). The foundation of the existence and the computation of the Lyapunov exponents builds the multiplicative ergodic theorem, also known as Oseledec theorem [67]. Briefly, it states that for the product of the single spike Jacobians $\mathbf{D}\left(t_{s}\right)$, the long term Jacobian $\mathbf{T}=\mathbf{D}\left(t_{s}\right) \cdots \mathbf{D}(0)$ and $t_{s}>0$ the Oseledec matrix $\Lambda$ exists for almost all initial conditions:

$$
\Lambda=\lim _{t \rightarrow \infty}\left(\mathbf{T}^{*} \mathbf{T}\right)^{\frac{1}{2 t}}
$$

where $\mathbf{T}^{*}$ denotes the transpose of $\mathbf{T}$ and the logarithms of the eigenvalues of the Oseledec matrix (2.16) are the Lyapunov exponents $\lambda_{1} \geq \cdots \geq \lambda_{N}$. However, because of the ill-conditioning of the Oseledec matrix (2.16), due to the exponential growth and decay of the eigenvalues, the standard procedure for the numerical calculation of the Lyapunov exponents [68, 69] exploits a different 
part of the Oseledec theorem. The exact Lyapunov exponents are equivalently defined for almost all initial conditions by the limits:

$$
\lambda_{i}=\lim _{t \rightarrow \infty} \frac{1}{t} \log \|\mathbf{T} \delta \vec{x}\| \quad \text { if } \delta \vec{x} \in E_{i} \backslash E_{i+1},
$$

where the $E_{i}$ 's are a valid decomposition of the phase space corresponding to subspaces spanned by the eigenvectors associated to the eigenvalues $\leq \exp \left(\lambda_{i}\right)$ of the Oseledec matrix. The vectors $\overrightarrow{\delta x}$ are normalized. In particular, Eq. (2.17) means that for almost all perturbations, namely $\delta \vec{x} \notin E_{2}$, the limit is the largest Lyapunov exponent.

An analytic estimation of the largest Lyapunov exponents should be possible for some special cases, such as a fully synchronous periodic network state. In this case, the symmetric Oseledec matrix (2.16) can be written down and the logarithms of the eigenvalues can be assessed with, e.g., the Gershgorin theorem [89].

In the more general case, including partially synchronous, quasiperiodic and chaotic states, a further analytic calculation of even the largest Lyapunov exponent is very difficult. For example, in an irregular asynchronous network state, like the balanced state studied in this paper, the spiking neuron differs for every spike in the network. Since the structure of the single spike Jacobians depends on the spiking neuron, already the structure will change considerably for every spike. The Oseledec matrix (2.16), is then determined by the product of (generally) noncommuting single spike Jacobians (2.13). This makes a further analytic calculation of the Lyapunov exponents in general rather difficult. They can typically only be computed numerically. We were, however, able to derive a random matrix approximation of the mean Lyapunov exponent in the balanced state, as discussed below.

This completes the derivation of the general approach to calculate the full spectrum of Lyapunov exponents in spiking neuron networks. We will now continue with the discussion of the balanced state and the theta neuron model before we can present the results of this approach for such networks in Section (2.6).

\subsection{The Balanced State in Sparse Random Networks}

We will study networks of $N$ spiking neurons arranged on directed Erdös-Rényi random graphs with average indegree $K$. This means that a connection between any pair of neurons is established with the probability $p=K / N$, where $1 \ll K \ll N$. Such random networks are intended to model, e.g., small units of cortical networks in which a spatially organized topology can be neglected.

These networks can exhibit a balanced state that resembles many features of cortical spiking activity. In the balanced state, asynchronous irregular firing activity of the neurons arises from strong input fluctuations that are of the same order of magnitude as the mean input current. This is a result of the dynamic balance of excitation and inhibition. It emerges robustly in random networks if the coupling between neurons exhibits a specific square root scaling $J_{i j} \propto 1 / \sqrt{K}[2,3]$. This scaling assures that the input fluctuations do not vanish in the large connectivity limit. The constant external currents $I_{i}^{\text {ext }}$ were chosen to obtain a target average firing rate $\bar{v}$. From the balance of excitation and inhibition, this target firing rate can be well estimated if the external currents scale as $I_{i}^{\text {ext }} \propto \sqrt{K}$. With these scaldings, neuron model-independent characteristics of the balanced state can be well approximated. We will derive these next for networks with just one population of inhibitory neurons and for networks consisting of excitatory and inhibitory neurons. 


\subsubsection{Inhibitory Networks}

In inhibitory networks, the balanced state emerges from the recurrent inhibition balancing the external excitation. The existence of a balanced state fixed point follows from the network-averaged input current. The input currents (2.2) are composed of constant external currents $I_{i}^{\text {ext }}=\sqrt{K} I_{0}$ and inhibitory pulses of strength $J_{i j} \tau_{\mathrm{m}}=-J_{0} \tau_{\mathrm{m}} / \sqrt{K}$. In the asynchronous balanced state, these are received at an average input rate $K \bar{v}$, from $K$ presynaptic neurons with an average firing rate $\bar{v}$ that is constant in time. Thus the mean input current can be written as

$$
\bar{I} \approx \sqrt{K}\left(I_{0}-J_{0} \bar{v} \tau_{\mathrm{m}}\right)
$$

In the large connectivity limit, self-consistency requires the balance of excitation and inhibition: If $\lim _{K \rightarrow \infty}\left(I_{0}-J_{0} \bar{v} \tau_{\mathrm{m}}\right)>0$ the mean current $\bar{I}$ would diverge to $\infty$ and the neurons would fire at their maximal rate. The resulting strong inhibition would break the inequality, leading to a contradiction. If $\lim _{K \rightarrow \infty}\left(I_{0}-J_{0} \bar{v} \tau_{\mathrm{m}}\right)<0$ the mean current $\bar{I}$ would diverge to $-\infty$ and the neurons would be silent. The resulting lack of inhibition again breaks the inequality. The large $K$-limit is self-consistent if $\lim _{K \rightarrow \infty}\left(I_{0}-J_{0} \bar{v} \tau_{\mathrm{m}}\right)=0$. Thus, the average firing rate in the balanced networks is approximately given by the balance equation:

$$
\bar{v}=\frac{I_{0}}{J_{0} \tau_{\mathrm{m}}}+\mathscr{O}\left(\frac{1}{\sqrt{K}}\right) .
$$

The magnitude of current fluctuations in sparse inhibitory networks can also be well approximated. Assuming that inputs from different presynaptic neurons are weakly correlated, the compound input spike trains can be modeled by a Poisson process with rate $K \bar{v}$. The input current auto-correlation then reads

$$
\begin{aligned}
C(\tau) & =\langle\delta I(t) \delta I(t+\tau)\rangle_{t} \\
& \approx\left(\frac{J_{0} \tau_{\mathrm{m}}}{\sqrt{K}}\right)^{2} K \bar{v} \int \delta(t-s) \delta(t+\tau-s) \mathrm{d} s \\
& =J_{0}^{2} \bar{v} \tau_{\mathrm{m}}^{2} \delta(\tau)
\end{aligned}
$$

Thus, the fluctuations can be described as delta-correlated white noise of magnitude $\sigma^{2}=J_{0}^{2} \bar{v} \tau_{\mathrm{m}}$. This is independent of $K$ and therefore does not vanish in the large $K$-limit.

\subsubsection{Excitatory-Inhibitory Networks}

In excitatory-inhibitory networks, the balanced state with finite firing rates in both populations can be inferred from a matrix equation of the mean currents, similar to Eq. (2.18):

$$
\begin{aligned}
\overline{\mathbf{I}} & =\sqrt{K}(\mathbf{I}+\mathbf{J} \cdot \bar{v}) \\
\left(\begin{array}{c}
\bar{I}_{E} \\
\bar{I}_{I}
\end{array}\right) & =\sqrt{K}\left[\left(\begin{array}{c}
E_{0} \\
I_{0}
\end{array}\right)+\left(\begin{array}{cc}
J_{E E} & -J_{E I} \\
J_{I E} & -J_{I I}
\end{array}\right) \cdot\left(\begin{array}{c}
\bar{v}_{E} \tau_{\mathrm{m}} \\
\bar{v}_{I} \tau_{\mathrm{m}}
\end{array}\right)\right] .
\end{aligned}
$$

Here, we denoted $\bar{I}_{A}$ the mean input current, $A_{0}>0$ the external currents and $\bar{v}_{A}>0$ the average firing rate of neurons in population $A=\{E, I\}$. For the coupling strengths from neurons in population $A$ to $B$ we used $J_{B A}>0$. All neurons were assumed to have an equal number of incoming 
connections $K$ from both populations. In the large $K$-limit, self-consistency requires the term in brackets in Eq. (2.21) to vanish, $\lim _{K \rightarrow \infty} \mathbf{I}+\mathbf{J} \bar{v}=0$, for the same arguments as above for inhibitory networks. The difference to inhibitory networks is that there is an additional dynamic excitatory term. Therefore, the inhibitory coupling must balance the excitatory external currents plus the excitatory coupling term, yielding the following constraint:

$$
J_{E E} \bar{v}_{E}<J_{E I} \bar{v}_{I} \quad \text { and } J_{I E} \bar{v}_{E}<J_{I I} \bar{v}_{I} \text {. }
$$

Two additional cases compared to the inhibitory networks are possible here: i) an exploding solution $\bar{v}_{E} \rightarrow \infty, \bar{v}_{I} \rightarrow \infty$ and ii) a silent excitatory population $\bar{v}_{E}=0$ but an active inhibitory population $\bar{v}_{I} \neq 0$. We want to exclude both cases which yields additional constraints on the coupling matrix. The exploding solution can be excluded if the inhibitory couplings are stronger than the excitatory ones

$$
J_{E E}<J_{E I} \text { and } J_{I E}<J_{I I}
$$

A silent excitatory population would be a possible solution if $E_{0}<J_{E I} \bar{v}_{I} \tau_{\mathrm{m}}$ and $I_{0}=J_{I I} \bar{v}_{I} \tau_{\mathrm{m}}$. To exclude this, we require

$$
\frac{J_{E I}}{J_{I I}}<\frac{E_{0}}{I_{0}}
$$

The self-consistent solution $\lim _{K \rightarrow \infty} \mathbf{I}+\mathbf{J} \bar{v}=0$ again defines the population-averaged firing rates $\bar{v}=-\mathbf{J}^{-1} \mathbf{I}$. For the coupling matrix to be invertible, it is required that the determinant does not vanish, $|\mathbf{J}| \neq 0$. Then the unique solution of the firing rates in the balanced state is

$$
\begin{aligned}
& \bar{v}_{E}=-\frac{J_{E I} I_{0}-J_{I I} E_{0}}{|\mathbf{J}| \tau_{\mathrm{m}}} \\
& \bar{v}_{I}=-\frac{J_{E E} I_{0}-J_{I E} E_{0}}{|\mathbf{J}| \tau_{\mathrm{m}}} .
\end{aligned}
$$

From Eq. (2.24) follows that the numerator in (2.25a) is negative. From the fact that the firing rate of the excitatory population should be positive, we find that the determinant of the coupling matrix must be positive: $|J|=-J_{E E} J_{I I}+J_{I E} J_{E I}>0$, thus

$$
J_{E E} J_{I I}<J_{I E} J_{E I}
$$

From $2.25 \mathrm{~b}$ and the fact that rate of the inhibitory population must be positive as well follows that

$$
\frac{J_{E E}}{J_{I E}}<\frac{E_{0}}{I_{0}} .
$$

Summarizing Eq. (2.22)-(2.24), (2.26) and (2.27), the balanced state emerges self-consistently if

$$
\frac{J_{E E}}{J_{E I}}<\frac{J_{I E}}{J_{I I}}<\min \left\{1, \frac{\bar{v}_{I}}{\bar{v}_{E}}\right\} \text { and } \frac{J_{E E}}{J_{I E}}<\frac{J_{E I}}{J_{I I}}<\frac{E_{0}}{I_{0}} .
$$

Putting this in words means that the ratio of the magnitudes of the incoming excitatory to inhibitory coupling must be smaller in the excitatory population than in the inhibitory population with an upper limit given by the minimum of 1 and the ratios of the population firing rates. The ratio of the outgoing excitatory to inhibitory coupling must also be smaller in the excitatory population than in the inhibitory population with an upper limit given by the external currents of the two populations. 
In our investigation, we want to compare the dynamics of the balanced state in excitatoryinhibitory and inhibitory networks. For a quantitative comparison, the firing statistics in both types of networks should be identical. This is accomplished if the fluctuations of the input currents have the same magnitude. Analogously to the derivation of the auto-correlation of the input currents in inhibitory networks, Eq. (2.20), the input currents in excitatory-inhibitory networks also display delta-correlated white noise with variance $\sigma^{(2)}=\mathbf{J}^{(2)} \bar{v}$, where ${ }^{(2)}$ denotes the square of the elements. We have chosen identical population-averaged firing rates $\bar{v}_{E}=\bar{v}_{I}=\bar{v}$, which yield

$$
\begin{aligned}
\sigma_{E}^{2} & =\left(J_{E E}^{2}+J_{E I}^{2}\right) \bar{v} \tau_{\mathrm{m}} \\
\sigma_{I}^{2} & =\left(J_{I I}^{2}+J_{I E}^{2}\right) \bar{v} \tau_{\mathrm{m}} .
\end{aligned}
$$

In order to satisfy identical magnitudes of input fluctuations $\sigma_{E}^{2}=\sigma_{I}^{2}=\sigma^{2}=J_{0}^{2} \bar{v} \tau_{\mathrm{m}}$ in both types of networks, we require

$$
J_{0}^{2}=J_{E E}^{2}+J_{E I}^{2}=J_{I I}^{2}+J_{I E}^{2}
$$

This is fulfilled by

$$
\mathbf{J}=\frac{J_{0}}{\sqrt{K}}\left(\begin{array}{cc}
\eta \varepsilon & -\sqrt{1-(\eta \varepsilon)^{2}} \\
\varepsilon & -\sqrt{1-\varepsilon^{2}}
\end{array}\right),
$$

where $\varepsilon$ activates the excitatory-inhibitory feedback loops in the networks and $\eta=J_{E E} / J_{I E}$ defines the ratio of the excitatory coupling of the excitatory and the inhibitory neurons. Note that for $\eta=1$ the determinant of the coupling matrix vanishes and the there is no unique solution of the firing rates (2.25). The derived balance condition for the coupling matrix, (2.28), requires:

$$
\begin{aligned}
\frac{\eta \varepsilon}{\sqrt{1-(\eta \varepsilon)^{2}}} & <\frac{\varepsilon}{\sqrt{1-\varepsilon^{2}}}<1 \\
\frac{1}{\eta \varepsilon}-1 & >\frac{1}{\varepsilon}-1>1 \\
\eta \varepsilon & <\varepsilon<\sqrt{\frac{1}{2}}
\end{aligned}
$$

which is satisfied for $\eta<1$ and $\varepsilon<0.7$. Finally, this sets the appropriate coupling matrix (2.31) to study excitatory-inhibitory networks with the same statistics as in inhibitory networks.

\subsection{Networks of Theta neurons}

As an example, our approach is applied to sparse networks of theta neurons. The theta neuron model, also known as the Ermentrout-Kopell model is the normal form of saddle-node bifurcations on limit cycles (SNIC) and the canonical model of type I excitable membranes [83-85]. Incorporating a dynamic action potential generation as in real neurons, the theta neuron model is biologically quite realistic despite its simple mathematical form.

The theta neuron model is the phase representation of the quadratic integrate and fire model. The quadratic integrate and fire model obeys $F\left(V_{i}\right)=V_{i}^{2}$ in Eq. (2.1) and has finite reset and threshold values. The theta neuron model is obtained by the transformation $V_{i}=\tan \left(\theta_{i} / 2\right)$ with $\theta_{i} \in[-\pi, \pi]$. The phases $\theta_{i}$ describe the neurons' phases defined on the unit circle. Spikes are emitted whenever the phases cross $\pi \rightarrow-\pi$ (Fig. 2.1 A). The governing differential equation of the theta neuron 

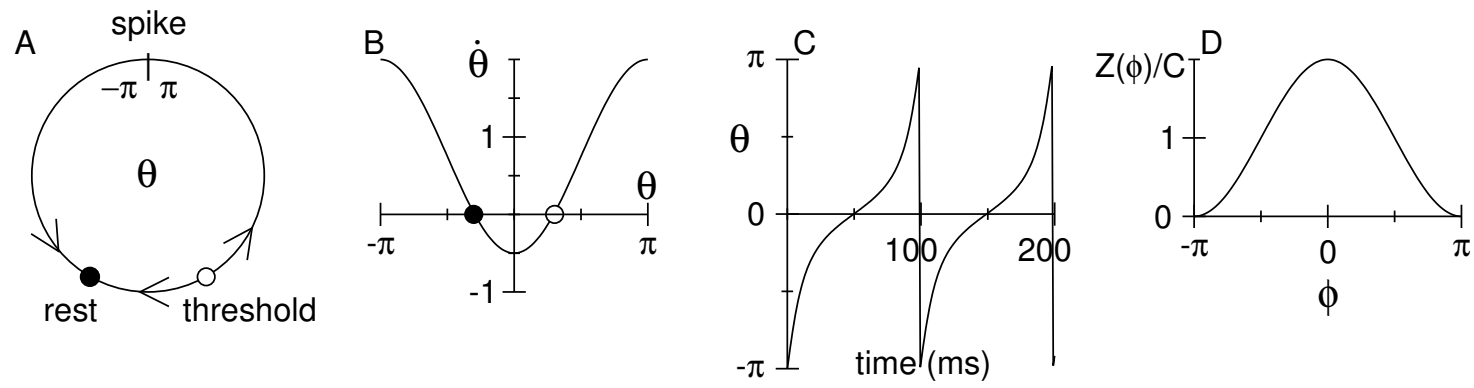

Figure 2.1 - The theta neuron model: (A) Schematic picture in the excitable state for negative input current, the two fixed points coalesce for increasing input current and the neuron oscillates, action potentials (spikes) occur when theta crosses pi, (B) phase plane in the excitable state for negative input current, Eq. 2.33, (C) periodic action potential generation in the oscillatory state for positive input current, (D) infinitesimal phase-response curve $Z(\phi) / C=1+\cos \phi$ with $C \rightarrow 0$, Eq. 2.39].

model reads

$$
\tau_{\mathrm{m}} \frac{\mathrm{d} \theta_{i}}{\mathrm{~d} t}=\left(1-\cos \theta_{i}\right)+\left(I_{i}(t)-I_{T}\right)\left(1+\cos \theta_{i}\right),
$$

with the rheobase current $I_{T}=0.25$. It is depicted in Fig. $2.1 \mathrm{~B}$ for a negative input current and a representative action potential trace of a free-running neuron receiving a constant positive input current is displayed in Fig. 2.1C.

In the studied networks, the synaptic input current $I_{i}(t)$, Eq. (2.2), can be rewritten without the explicit time dependence. The transformation $\delta\left(\theta_{j}(t)-\pi\right)=\sum_{s} \delta\left(t-t_{j}^{(s)}\right) /\left|\dot{\theta}_{j}\left(t_{j}^{(s)}\right)\right|$ and the fact that $\tau_{\mathrm{m}} \dot{\theta}_{j}\left(t_{j}^{(s)}\right)=1-\cos \pi=2$ at the spike times lead to

$$
I_{i}(t)=I_{T}+\sqrt{K} I_{i}^{\mathrm{ext}}+2 \sum_{j \in \operatorname{pre}(i)} \frac{J_{i j}}{\sqrt{K}} \delta\left(\theta_{j}(t)-\pi\right) .
$$

Thus, networks of theta-neurons are conveniently described by an autonomous system of ordinary differential equations. Furthermore, they allow for a mathematically sound description of networks with excitatory coupling. In networks with excitatory coupling, a natural problem arises with threshold neurons that are sensitive to inputs close to their own spike. Then, an excitatory input pulse can result in a suprathreshold state and the question about the extra suprathreshold charge arises. It was for example proposed to introduce a partial reset in this case [90]. However, neuron models with a phase-response approaching zero near the spike, such as the theta neuron model, circumvent this problem (Fig. 2.1C). Then the study of excitatory networks is straightforward from inhibitory networks without mathematical difficulties.

Another equivalent phase representation of the theta neuron model can be even more convenient, namely the phi-representation. In this representation, obtained by $V_{i}(t)=\left(K^{\frac{1}{2}} I_{i}^{\text {ext }}\right)^{\frac{1}{2}} \tan \left(\phi_{i}(t) / 2\right)$ with $\phi_{i} \in[-\pi, \pi]$, the neurons' phases have constant phase velocities between spike events. The phase updates at spike reception are described by the phase transition curve, respectively the phaseresponse curve. This representation of the theta neuron model is used in the numerical simulations.

The theta neuron model has the big advantage of being exactly solvable. Between two spikes $t_{s}$ and $t_{s+1}$ in the network, all neurons only receive constant external inputs and the evolution functions in the voltage representation $V_{i}$, theta representation $\theta_{i}=2 \arctan \left(V_{i}\right)$ and phi representation 
$\phi_{i}=2 \arctan \left(V_{i} /\left(K^{\frac{1}{2}} I_{i}^{\text {ext }}\right)^{\frac{1}{2}}\right)$ with the phase velocity $\omega_{i}=2\left(K^{\frac{1}{2}} I_{i}^{\text {ext }}\right)^{\frac{1}{2}}$ are

$$
\begin{aligned}
f\left(V_{i}\left(t_{s}\right), t_{s+1}-t_{s}\right) & =\frac{\omega_{i}}{2} \tan \left(\arctan \left(\frac{V_{i}\left(t_{s}\right)}{\omega_{i} / 2}\right)+\frac{\omega_{i}}{2} \frac{t_{s+1}-t_{s}}{\tau_{\mathrm{m}}}\right) \\
f\left(\theta_{i}\left(t_{s}\right), t_{s+1}-t_{s}\right) & =2 \arctan \left(\frac{\omega_{i}}{2} \tan \left(\arctan \left(\frac{\tan \left(\theta_{i}\left(t_{s}\right) / 2\right)}{\omega_{i} / 2}\right)+\frac{\omega_{i}}{2} \frac{t_{s+1}-t_{s}}{\tau_{\mathrm{m}}}\right)\right) \\
f\left(\phi_{i}\left(t_{s}\right), t_{s+1}-t_{s}\right) & =\phi_{i}\left(t_{s}\right)+\omega_{i} \frac{t_{s+1}-t_{s}}{\tau_{\mathrm{m}}} .
\end{aligned}
$$

When receiving a spike of strength $J_{i^{*} j^{*}} / \sqrt{K}$ from the spiking neuron $j^{*}$ the postsynaptic neurons $i^{*}$ are updated with

$$
\begin{aligned}
& g\left(V_{i^{*}}\right)=V_{i^{*}}+\frac{J_{i^{*} j^{*}}}{\sqrt{K}} \\
& g\left(\theta_{i^{*}}\right)=2 \arctan \left(\tan \frac{\theta_{i^{*}}}{2}+\frac{J_{i^{*} j^{*}}}{\sqrt{K}}\right) \\
& g\left(\phi_{i^{*}}\right)=2 \arctan \left(\tan \frac{\phi_{i^{*}}}{2}+\frac{J_{i^{*} j^{*}}}{\left(K^{\frac{3}{2}} I^{\text {ext }}\right)^{\frac{1}{2}}}\right) .
\end{aligned}
$$

In the simulations we used the phi representation with constant phase velocity $\omega_{i}=2\left(K^{\frac{1}{2}} I_{i}^{\text {ext }}\right)^{\frac{1}{2}}$ and the phase transition curve $g\left(\phi_{i}\right)$, respectively the phase response curve $Z\left(\phi_{i}\right)=g\left(\phi_{i}\right)-\phi_{i}$. With these, the single spike Jacobian (2.15) for theta neuron networks reads

$$
D_{i j}\left(t_{s}\right)= \begin{cases}d_{i^{*}}\left(t_{s+1}^{-}\right) & \text {for } i=j=i^{*} \\ \sqrt{\frac{l_{i *}^{\text {ext }}}{I_{j^{*}}^{\text {xxt }}}}\left(1-d_{i^{*}}\left(t_{s+1}^{-}\right)\right) & \text {for } i=i^{*} \text { and } j=j^{*} \\ \delta_{i j} & \text { otherwise, }\end{cases}
$$

where $j^{*}$ denotes the spiking neuron in the considered interval firing at time $t_{s+1}, i^{*} \in \operatorname{post}\left(j^{*}\right)$ are the spike receiving neurons, $\delta_{i j}$ is the Kronecker symbol. The elements $d_{i^{*}}\left(t_{s+1}^{-}\right)$expressed in the three equivalent representations are:

$$
\begin{aligned}
d_{i^{*}}\left(t_{s+1}^{-}\right) & =\frac{\left(\tan \left(\phi_{i^{*}}\left(t_{s+1}^{-}\right) / 2\right)\right)^{2}+1}{\left(\tan \left(\phi_{i^{*}}\left(t_{s+1}^{-}\right) / 2\right)+J_{i^{*} j^{*}} /\left(K^{\frac{3}{2}} I_{i^{*}}^{\text {ext }}\right)^{\frac{1}{2}}\right)^{2}+1} \\
& =\frac{\left(\tan \left(\theta_{i^{*}}\left(t_{s+1}^{-}\right) / 2\right)\right)^{2}+\sqrt{K} I_{i^{*}}^{\text {ext }}}{\left(\tan \left(\theta_{i^{*}}\left(t_{s+1}^{-}\right) / 2\right)+J_{i^{*} j^{*}} / \sqrt{K}\right)^{2}+\sqrt{K} I_{i^{*}}^{\text {ext }}} \\
& =\frac{\left(V_{i^{*}}\left(t_{s+1}^{-}\right)\right)^{2}+\sqrt{K} I_{i^{*}}^{\text {ext }}}{\left(V_{i^{*}}\left(t_{s+1}^{-}\right)+J_{i^{*} j^{*}} / \sqrt{K}\right)^{2}+\sqrt{K} I_{i^{*}}^{\text {ext }}} .
\end{aligned}
$$

They are evaluated at the neurons states just before spike reception $x_{i^{*}}\left(t_{s+1}^{-}\right)=f\left(x_{i^{*}}\left(t_{s}\right), t_{s+1}-t_{s}\right)$. 
The single spike Jacobians (2.37) of the theta neuron networks will be used to numerically calculate the spectrum of Lyapunov exponents in the standard procedure as explained below.

The phi representation draws the connection back to the more general description of our method using the phase-response curve. The phase response curve $Z\left(\phi_{i}\right)$ can also be expressed with the infinitesimal phase-response curve for arbitrarily small coupling strength (Fig. 2.1D). In our case, this would correspond to the large-K limit, where the effective coupling $C_{i j}=J_{i j} /\left(K^{\frac{3}{2}} I_{i}^{\text {ext }}\right)^{\frac{1}{2}}$ becomes vanishingly small. The Taylor expansion of the phase-response curve is

$$
\begin{gathered}
Z\left(\phi_{i}\right) \quad=2 \arctan \left(\tan \frac{\phi_{i}}{2}+C_{i j}\right)-\phi_{i} \\
\stackrel{C_{i j} \rightarrow 0}{\simeq} C_{i j}\left(1+\cos \phi_{i}\right)+\mathscr{O}\left(C_{i j}^{2}\right),
\end{gathered}
$$

and the derivative $Z^{\prime}\left(\phi_{i}\right) \simeq-C_{i j} \sin \phi_{i}+\mathscr{O}\left(C_{i j}^{2}\right)$. The Jacobian 2.15) expressed with the infinitesimal phase-response curve of the theta neuron model thus reads

$$
D_{i j}\left(t_{s}\right)= \begin{cases}1-C_{i^{*} j^{*}} \sin \left(\phi_{i^{*}}\left(t_{s+1}^{-}\right)\right) & \text {for } i=j=i^{*} \\ \frac{\omega_{i^{*}}}{\omega_{j^{*}}} C_{i^{*} j^{*}} \sin \left(\phi_{i^{*}}\left(t_{s+1}^{-}\right)\right) & \text {for } i=i^{*} \text { and } j=j^{*} \\ \delta_{i j} & \text { otherwise. }\end{cases}
$$

\subsubsection{Random Matrix Approximation of Mean Lyapunov Exponent}

From the single spike Jacobian (2.37), we derived a random matrix approximation of the mean Lyapunov exponent $\bar{\lambda}=\frac{1}{N} \sum_{i=1}^{N} \lambda_{i}$. The mean Lyapunov exponent describes the rate of phase space compression, captured by the determinant of the long term Jacobian $\mathbf{T}=\mathbf{D}\left(t_{s}\right) \cdots \mathbf{D}(0)$ :

$$
\begin{aligned}
\bar{\lambda} & =\frac{1}{N} \lim _{s \rightarrow \infty} \frac{1}{t_{s}} \ln (\operatorname{det} \mathbf{T}) \\
& =\frac{1}{N} \lim _{s \rightarrow \infty} \frac{1}{t_{s}} \sum_{p=1}^{s} \ln \left(\operatorname{det} \mathbf{D}\left(t_{p}\right)\right)
\end{aligned}
$$

The random matrix approximation is obtained by assuming the single spike Jacobians to be random matrices of the form (2.37) with independent and identically distributed random elements obtained from the function $d(V)$, Eq. (2.38c). The probability distribution of the random elements is determined by the stationary membrane potential distribution $P(V)$ in the network.

For inhibitory networks, the determinant of the random matrices can be approximated by $\operatorname{det} \mathbf{D}=$ $\prod_{i^{*}} d_{i^{*}} \approx d(V)^{K}$, since on average there are $K$ diagonal elements $d_{i^{*}}$, one for each postsynaptic neuron. We assume homogeneous coupling strengths $J_{i j} \equiv-J_{0}$ between connected neurons and identical external currents $I_{i}^{\text {ext }} \equiv I_{0}$ for all neurons in Eq. (2.34). The number of spike events per unit time is $\lim _{s \rightarrow \infty} \frac{1}{t_{s}} \sum_{p=1}^{s} 1=N \bar{v}$. Thus, in the random matrix approximation, the mean Lyapunov exponent for inhibitory networks becomes

$$
\begin{aligned}
\bar{\lambda} & \approx \frac{1}{N} N \bar{v} \int \ln \left(d(V)^{K}\right) P(V) \mathrm{d} V \\
& =K \bar{v} \int \ln \left(\frac{V^{2}+\sqrt{K} I_{0}}{\left(V-J_{0} / \sqrt{K}\right)^{2}+\sqrt{K} I_{0}}\right) P(V) \mathrm{d} V
\end{aligned}
$$




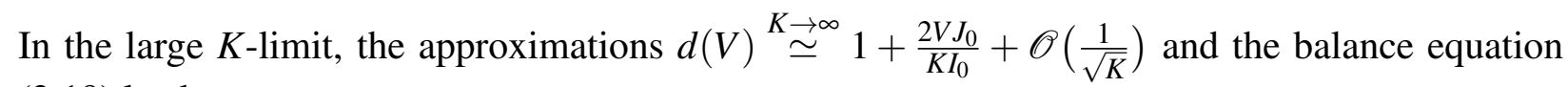
(2.19) lead to

$$
\bar{\lambda} \stackrel{K \rightarrow \infty}{\simeq} \frac{2\langle V\rangle}{\tau_{\mathrm{m}}}+\mathscr{O}\left(\frac{1}{\sqrt{K}}\right)
$$

where $\langle V\rangle$ denotes the average membrane potential.

For excitatory-inhibitory networks, the determinant of the random matrices can be approximated by $\operatorname{det} \mathbf{D} \approx d_{I I}(V)^{K_{I I}^{\text {out }}} d_{E I}(V)^{K_{E I}^{\text {out }}}$ at spike times of inhibitory neurons, and at spike times of excitatory neurons by $\operatorname{det} \mathbf{D} \approx d_{E E}(V)^{K_{E E}^{\text {out }}} d_{I E}(V)^{K_{I E}^{\text {out }}}$. The Jacobian elements are denoted $d_{X Y}$ and the mean outdegrees (average number of postsynaptic neurons) are $K_{X Y}^{\text {out }}$ with $X$ and $Y$ representing the excitatory $(E)$ and inhibitory $(I)$ populations. On average there are $N_{X} \bar{v}_{X}$ spikes in each population. This leads to the random matrix approximation of the mean Lyapunov exponent in excitatory-inhibitory networks

$$
\begin{aligned}
\bar{\lambda}= & \frac{N_{I} \bar{v}_{I}}{N}\left(K_{I I}^{\text {out }} \int \ln \left(d_{I I}(V)\right) P_{I}(V) \mathrm{d} V+K_{E I}^{\text {out }} \int \ln \left(d_{E I}(V)\right) P_{E}(V) \mathrm{d} V\right)+ \\
& \frac{N_{E} \bar{v}_{E}}{N}\left(K_{I E}^{\text {out }} \int \ln \left(d_{I E}(V)\right) P_{I}(V) \mathrm{d} V+K_{E E}^{\text {out }} \int \ln \left(d_{E E}(V)\right) P_{E}(V) \mathrm{d} V\right),
\end{aligned}
$$

where $P_{X}(V)$ denotes the membrane potential distributions of either population. In our simulations, the coupling strengths were chosen such that the input current statistics in both populations were identical in the balanced state. This led to identical membrane potential distributions $P_{E}(V)=$ $P_{I}(V) \equiv P(V)$. The number of inhibitory neurons was $N_{I}=0.2 N$ and the number of excitatory neurons $N_{E}=0.8 N$. The average firing rates were identical in both populations $\bar{v}_{E}=\bar{v}_{I} \equiv \bar{v}$. The average indegrees were all $K$, leading to the outdegrees $K_{X Y}^{\text {out }}=K N_{X} / N_{Y}$. This can be summarized in the approximated mean Lyapunov exponent for the excitatory-inhibitory networks

$$
\bar{\lambda}=K \bar{v} \int\left(0.2 \ln d_{I I}(V)+0.8 \ln d_{E I}(V)+0.8 \ln d_{E E}(V)+0.2 \ln d_{I E}(V)\right) P(V) \mathrm{d} V,
$$

with the elements

$$
d_{X Y}(V)=\frac{V^{2}+\sqrt{K} I_{X}}{\left(V+J_{X Y} / \sqrt{K}\right)^{2}+\sqrt{K} I_{X}} .
$$

Taking the large $K$-limit leads to

$$
\begin{aligned}
\bar{\lambda} \stackrel{K \rightarrow \infty}{\simeq}-2\langle V\rangle \bar{v}\left(0.2 \frac{J_{I I}+J_{I E}}{I_{I}}+0.8 \frac{J_{E I}+J_{E E}}{I_{E}}\right) \\
=\frac{2\langle V\rangle}{\tau_{\mathrm{m}}}+\mathscr{O}\left(\frac{1}{\sqrt{K}}\right) .
\end{aligned}
$$

In the last step we used the balance equation $\bar{v}=\bar{v}_{X}=-I_{X} /\left(\sum_{Y=\{E, I\}} J_{X Y} \tau_{\mathrm{m}}\right)$. With the above assumptions, the mean Lyapunov exponent in excitatory-inhibitory networks is the same as in inhibitory networks.

We have tested the derived random matrix approximations of the mean Lyapunov exponent in 


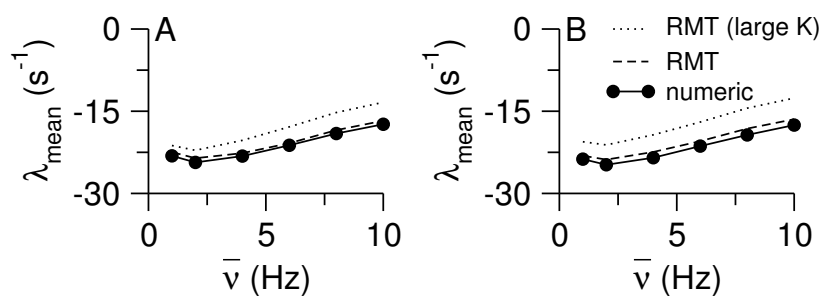

Figure 2.2 - Comparison of mean Lyapunov exponent in random matrix approximations and from numerical simulations in balanced theta neuron networks. (A) Inhibitory networks with $N=2000$, (B) excitatory-inhibitory networks with $N_{I}=2000, N_{E}=8000, \eta=0.9, \varepsilon=0.3$, straight lines: numerical simulations, dashed lines: random matrix approximations with full membrane potential distributions Eq. 2.42) and Eq. 2.45, dotted lines: random matrix approximation in the large $K$-limit Eq. (2.43) and Eq. 2.47), (other parameters: $\bar{v}=1 \mathrm{~Hz}, K=100, J_{0}=1, \tau_{\mathrm{m}}=10 \mathrm{~ms}$ ).

inhibitory networks Eq. (2.42) and in excitatory-inhibitory networks Eq. (2.45) and the large- $K$ limits, Eq. (2.43) and (2.47), with the results from simulations (Fig. 2.2). They are in very good agreement, indicating the validity of the random matrix approximation. This is probably the case because of the commutativity of the determinants of the Jacobians, a property that is not expected to hold for the product of the Jacobians in general, which eigenvalues define the individual Lyapunov exponents.

\subsubsection{Numerical Procedure and Convergence}

All simulations were run in an event-based fashion following Ref. [51, 54, 58], where the exact map (2.3) was iterated from spike to spike in the phi representation of the theta neuron model with homogeneous coupling strengths and homogeneous external currents for all neurons in each population. To calculate the next spike time in the network it was sufficient to find the neuron with the largest phase in either population and then calculate its next spike time

$$
t_{s+1}=t_{s}+\min _{i}\left\{\frac{\pi-\phi_{i}\left(t_{s}\right)}{\omega_{i}}\right\}
$$

since the external currents of the neurons were identical in either population. In the case of two populations, the minimum of the two calculated next spike times would be the next spike time in the network. Then, all neurons' phases were evolved until the next spike time using Eq. 2.35c) and the spike receiving neurons updated with Eq. (2.36c). These three steps compose one iteration and give numerically exact spike times and phases of the neurons.

With the exact phases of the neurons before spike reception, the single spike Jacobians 2.37) were evaluated using Eq. (2.38a). These were used to numerically calculate all Lyapunov exponents in the standard procedure [68,69]. After a warmup of the network dynamics, of typically 100 spikes per neuron on average, we started with a random $N$-dimensional orthonormal system that was evolved in each iteration with the single spike Jacobian. After some iterations of about $N / K$ spikes, the evolved vectors were reorthonormalized with the Gram-Schmidtorthonormalization procedure, yielding the norms of the orthogonalized vectors $n_{i}\left(t_{s}\right)$ and the orthonormal system to be used in the next iteration. After a short warmup of the orthonormal system of about one spike per neuron, these norms were used to calculate the $N$ Lyapunov exponents $\lambda_{i}=\lim _{p \rightarrow \infty} \frac{1}{t_{p}} \sum_{s=1}^{p} \log n_{i}\left(t_{s}\right)$. 

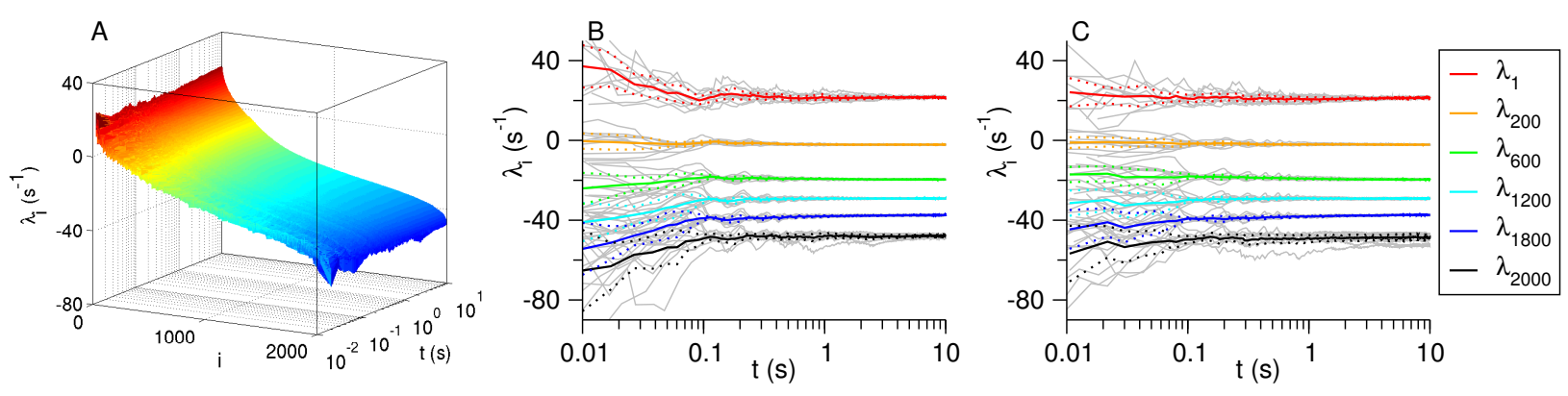

Figure 2.3 - Convergence of Lyapunov spectra versus time in inhibitory networks. (logarithmic time scale) (A) Convergence of Lyapunov spectrum for one initial condition, (B) grey lines: some Lyapunov exponents for ten different initial phases, straight color lines: averages, dotted color lines: averages \pm double standard errors, (C) as in (B) but for different network realizations in each run (parameters: $N=2000$, $\left.\bar{v}=1 \mathrm{~Hz}, K=100, J_{0}=1, \tau_{\mathrm{m}}=10 \mathrm{~ms}\right)$.

An example code for MATLAB ${ }^{\circledR}$ illustrating the principle steps to calculate the Lyapunov spectra is provided Appendix B. All full calculations were performed in custom code written in $\mathrm{C}++$ with double precision. The GNU Scientific Library (GSL) was used for the random number generator (Mersenne-Twister), the Automatically Tuned Linear Algebra Software (ATLAS) for matrix multiplications in the Gram-Schmidt procedure and the Message Passing Interface (MPI) for the parallel implementation of the simulations. The sparseness of the networks was efficiently used for the storage of the coupling matrices, the updates of the postsynaptic neurons and the matrix multiplications of the orthonormal system with the sparse single spike Jacobians. For the reorthonormalization, we chose a parallel recursive blocked version of the Gram-Schmidt procedure [91].

The Lyapunov spectra converged rather quickly over time to their asymptotic shape (Fig. 2.3. One should note that the non-converged Lyapunov exponents are meaningless (they do not reflect the local or finite-time Lyapunov exponents). The converged Lyapunov exponents capture the asymptotic network dynamics. Figure 2.3 A displays the convergence towards the full Lyapunov spectrum on logarithmic time scale. This calculation was repeated for different initial phases. Figure 2.3B shows the results of ten such runs for six of the Lyapunov exponents (grey lines), together with their averages $\lambda_{i}=\frac{1}{10} \sum_{\eta=1}^{10} \lambda_{i, r}$ (straight color lines) and confidence intervals (dotted color lines) of the double standard error $2 \triangle \lambda_{i}=2 \sqrt{\frac{1}{10} \sum_{\eta=1}^{10}\left(\lambda_{i, r}-\lambda_{i}\right)^{2}}$. Figure $2.3 \mathrm{C}$ shows the results of ten runs with different initial phases and different network realizations. The Lyapunov spectrum was independent of the initial phases as well as network realizations. Generally, all calculations of the Lyapunov spectra were repeated ten times with different initial phases and network realizations. Numerical errors were smaller than the symbol sizes in the presented figures.

The convergence of the largest Lyapunov exponents for different parameter sets in inhibitory and excitatory-inhibitory networks is depicted in Fig. 2.4 and Fig. 2.5, respectively. The evolution of the largest Lyapunov exponent was plotted versus the logarithm of the average number of spikes per neuron $S$ (the number of all spikes in the network divided by the number of neurons). $S=1$ means that every neuron has spiked approximately once. A characteristic scale is $S_{D}=\log (N) / \log (K)$, corresponding to the diameter of the random graph (the largest number of neurons on the shortest paths between any pair of neurons). In excitatory-inhibitory networks, the neurons have $K$ inhibitory presynaptic neurons and $K$ excitatory presynaptic neurons. Therefore we used $S_{D}=\log (N) / \log (2 K)$ in the excitatory-inhibitory networks. $S_{D}$ is the approximate number of spikes after which a perturbation of any neuron has influenced all others. This quantity is 

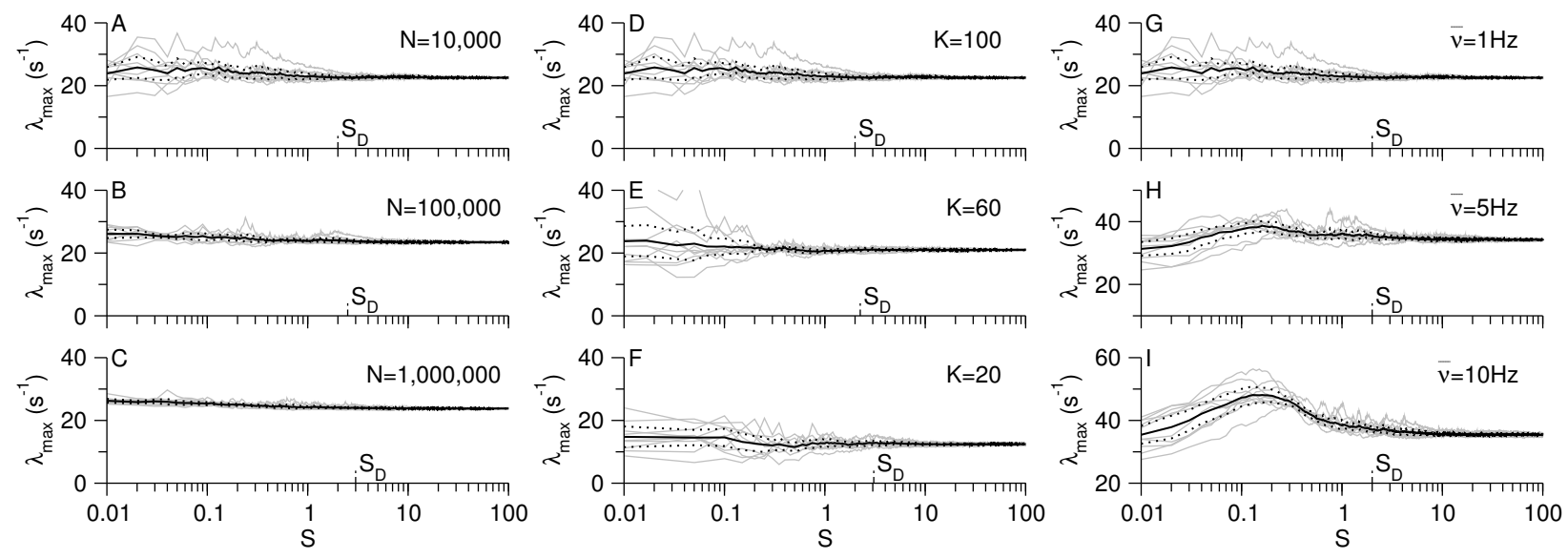

Figure 2.4 - Convergence of largest Lyapunov exponent in inhibitory networks versus average number of spikes per neuron $S \approx \bar{v} t$. (logarithmic scale) (A)-(C) Different network sizes $N(K=100, \bar{v}=1 \mathrm{~Hz})$, (D)-(F) different mean indegrees $K(N=10000, \bar{v}=1 \mathrm{~Hz}),(\mathrm{G})$-(I) different average firing rates $\bar{v}(N=$ $10000, K=100$ ), (grey lines: ten runs with different network realizations, black straight lines: averages of these ten runs, dotted lines: averages \pm double standard errors, $S_{D}=\log (N) / \log (K)$, other parameters: $J_{0}=1, \tau_{\mathrm{m}}=10 \mathrm{~ms}$ ).
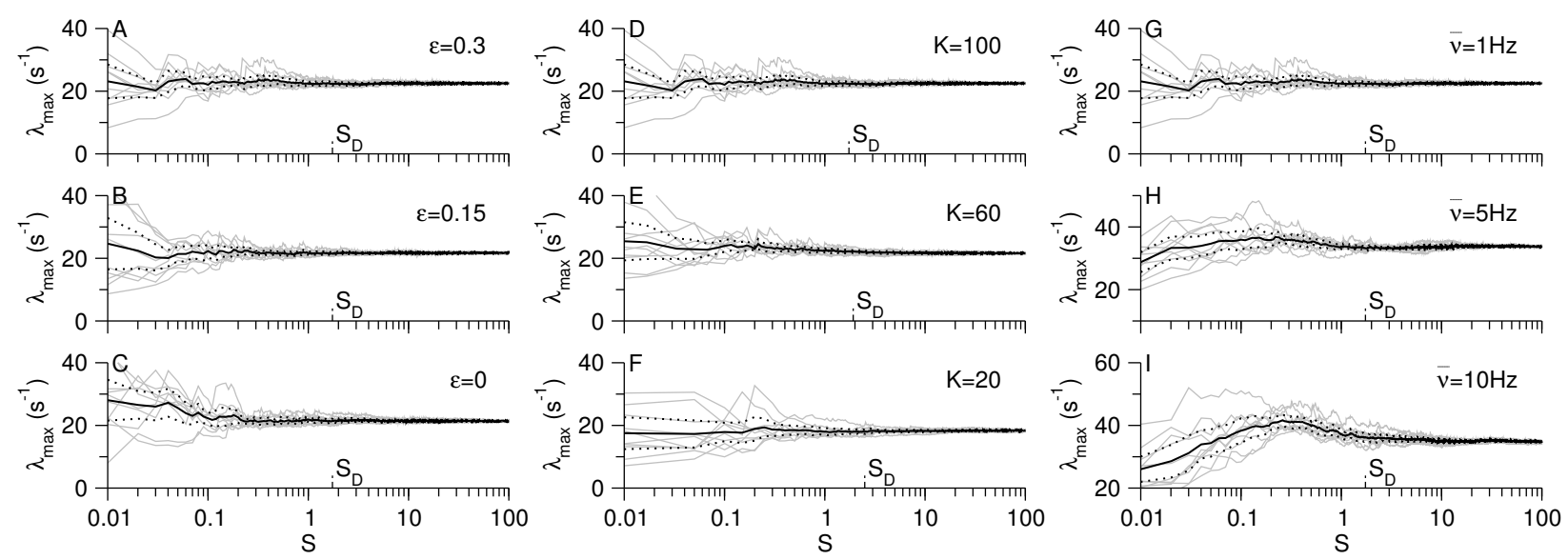

Figure 2.5 - Convergence of largest Lyapunov exponent in excitatory-inhibitory networks versus average number of spikes per neuron $S \approx \bar{v} t$. (logarithmic time scale) (A)-(C) Different excitatory-inhibitory feedback loop activation $\varepsilon(K=100, \bar{v}=1 \mathrm{~Hz})$, (D)-(F) different mean indegrees $K(\varepsilon=0.3, \bar{v}=1 \mathrm{~Hz})$, (G)-(I) different average firing rates $\bar{v}(\varepsilon=0.3, K=100)$, (grey lines: ten runs with different network realizations, black straight lines: averages of these ten runs, dotted lines: averages \pm double standard errors, $S_{D}=\log (N) / \log (2 K)$, other parameters: $\left.N_{E}=8000, N_{I}=2000, J_{0}=1, \tau_{\mathrm{m}}=10 \mathrm{~ms}\right)$. 
a reasonable scale for the convergence of the Lyapunov exponents in inhibitory and excitatoryinhibitory networks. The fluctuations of the largest Lyapunov exponents decreased similarly for varying parameters, e.g., number of synapses $K$, average firing rates $\bar{v}$ and excitatory loop activations $\varepsilon$ (Fig. 2.4, 2.5).

For increasing network sizes $N$, we observed decreasing fluctuations (Fig. 2.4A-C). This, together with the independence of the Lyapunov spectra of the network realizations, indicates selfaveraging of the Lyapunov spectra. Quantities that are self-averaging converge for large system sizes to the ensemble average. The Lyapunov spectrum of one realization of a large network is thus representative for the whole ensemble. Hence, averaging over many different network realizations is not necessary for large networks.

\subsection{Asynchronous States and Transitions to Synchrony}

So far, we have introduced the general approach for the numerically exact simulation and characterization of the dynamics of spiking neuron networks and discussed the methodological details of the balanced state and theta neuron networks in particular. Now, we are ready to present the results of a comprehensive analysis of the dynamics of theta neuron networks in the balanced state. These simple models provide valuable insight of the nature of real cortical circuits. We discuss the neurons' spike characteristics and their complex collective dynamics in both inhibitory and excitatory-inhibitory networks.

The balanced state of cortical networks is characterized by asynchronous irregular firing activity of the neurons. We measured the irregularity of individual neurons' spike sequences with the coefficient of variation

$$
\mathrm{cv}_{i}=\frac{\operatorname{STD}\left(T_{i}^{\mathrm{isi}}\right)}{\operatorname{MEAN}\left(T_{i}^{\mathrm{isi}}\right)}
$$

Here $T_{i}^{\text {isi }}$ denotes the interspike intervals of neuron $i, \operatorname{STD}(\ldots)=\sqrt{\left\langle\ldots{ }^{2}\right\rangle-\langle\ldots\rangle^{2}}$ is the standard deviation and $\operatorname{MEAN}(\ldots)=\langle\ldots\rangle$ is the mean. The angular brackets $\langle\ldots\rangle$ denote the time average. The asynchrony/synchrony of the neurons' firing activity across the population was determined with a synchrony measure following Ref. [92]

$$
\chi=\frac{\operatorname{STD}\left(\left[\phi_{i}\right]\right)}{\left[\operatorname{STD}\left(\phi_{i}\right)\right]}
$$

The brackets $[\ldots]=\frac{1}{N} \sum_{i=1}^{N} \ldots$ denote the ensemble average over the whole population of neurons. The synchrony measure 2.50) measures the variation of a macroscopic quantity (here the average of all neurons' phases) normalized with the average of the variations of the microscopic quantity (single neuron's phases). It ranges from 0 (full asynchrony) to 1 (full synchrony). Important for an asynchronous state is that the synchrony measure $\chi$ goes to zero as $1 / \sqrt{N}$. This was indeed the case in both types of networks in the balanced state as can be seen in Fig. 2.10 and 2.19, where the average $\mathrm{cv}=\left[\mathrm{cv}_{i}\right]$ and $\chi$ are presented along with the results of the network dynamics.

Figure 2.6 displays the general characteristics of the firing activity of neurons in both inhibitory and excitatory-inhibitory networks in the balanced state. Characteristic of the balanced state is the asynchronous and irregular spike activity of the neurons, visualized in the representative spike patterns of 40 randomly chosen neurons. The voltage traces of single neurons indicate the typical strong subthreshold fluctuations in the balanced state. Hence, the voltage distributions in the 

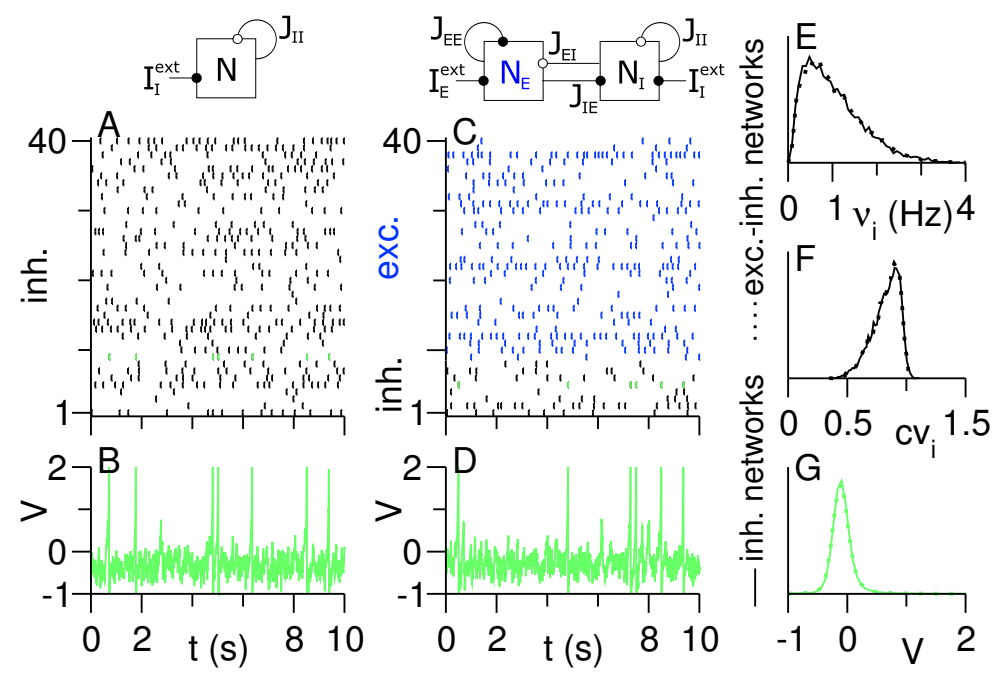

Figure 2.6 - Identical firing characteristics in inhibitory networks (left) and excitatory-inhibitory networks (middle) in the balanced state. (A),(C) Spike patterns of 40 randomly chosen neurons, (B),(D) voltage traces of one random neuron, (E) firing rate distributions, (F) coefficient of variation distributions and $(\mathrm{G})$ stationary voltage distributions for both types of networks, (parameters: $N=10000, K=100$, $\left.\bar{v}=1 \mathrm{~Hz}, J_{0}=1, \tau_{\mathrm{m}}=10 \mathrm{~ms}, \eta=0.9, \varepsilon=0.3, N_{E}=4 N_{I}\right)$.

networks were broad. The distributions of firing rates $v_{i}$ and coefficients of variation $\mathrm{cv}_{i}$ indicate substantial heterogeneities in the balanced networks. Moreover, these distributions were identical in both types of networks, due to the specific choice of coupling strengths, Eq. (2.31), that lead to identical input current statistics in the balanced state. This allows for a quantitative comparison of the dynamics of the two types of networks.

Although the balanced state emerged for a broad parameter range, two different transitions from the asynchronous to synchronous states were also observed in the theta neuron networks. In inhibitory networks, the neurons' activity synchronized upon increasing the connectivity $K$. Even though the spike sequences of individual neurons remained irregular, the population activity revealed a partially synchronous state for $K>K_{c} \approx 200$ (Fig. 2.7). In excitatory-inhibitory networks, the activity of neurons synchronized and became regular upon increasing the excitatory-inhibitory feedback loop strength $\varepsilon$ above a critical value $\varepsilon_{c}$ (Fig. 2.8). The latter depended on the ratio of the excitatory coupling $\eta=\frac{J_{E E}}{J_{I E}}$ between the excitatory and inhibitory neurons (Fig. 2.9). The excitatory interpopulation coupling $J_{I E}$ promoted this transition, whereas the excitatory intrapopulation coupling $J_{E E}$ hindered it. Since we here focus on the analysis of the asynchronous irregular balanced state, a high ratio $\eta=0.9$ is used throughout the rest of the paper. For this value, the transition to the synchronous state occurred at $\varepsilon_{c} \approx 0.5$.

\subsection{Dynamics of Inhibitory Networks}

Sparse inhibitory networks of theta neurons in the balanced state exhibit conventional deterministic chaos that is furthermore extensive (Fig. 2.10). We first examined the collective dynamics of sparse networks with fixed number of synapses per neuron $K$ and increasing network size (number of neurons) $N$. The spike statistics was independent of $N$. The decreasing synchrony measure $\chi \sim 1 / \sqrt{N}$ and the high coefficient of variation $\mathrm{cv} \approx 0.8$ demonstrate the typical asynchronous and irregular firing activity in these balanced networks (Fig. 2.10A,B). Importantly, the dynamics 

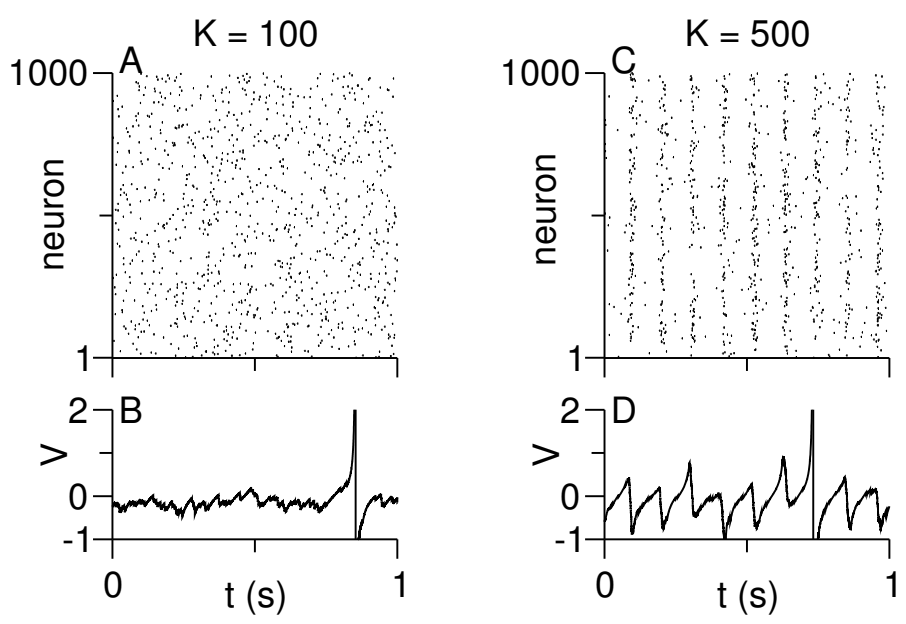

Figure 2.7 - Transition from asynchronous irregular state (left) to synchronous irregular state (right) in inhibitory networks. (A) Spike patterns of 1000 randomly chosen neurons for $K=100$, (B) voltage trace of one random neuron for $K=100,(\mathrm{C}),(\mathrm{D})$ as (A),(B) but for for $K=500$ (other parameters: $N=10000$, $\left.\bar{v}=1 \mathrm{~Hz}, J_{0}=1, \tau_{\mathrm{m}}=10 \mathrm{~ms}\right)$.
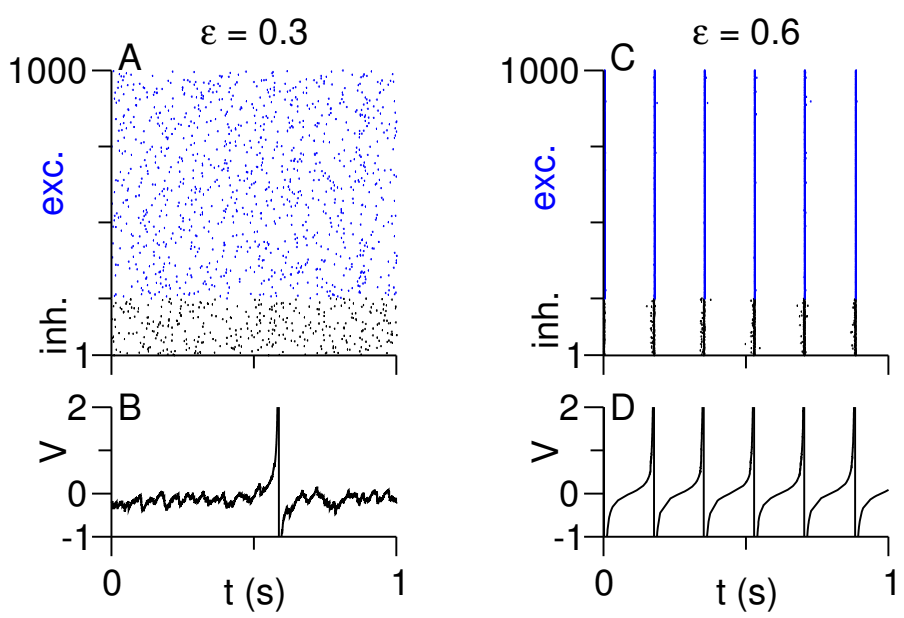

Figure 2.8 - Transition from asynchronous irregular state (left) to synchronous regular state (right) in excitatory-inhibitory networks. (A) Spike patterns of 1000 randomly chosen neurons for $\varepsilon=0.3$, (B) voltage traces of one random neuron for $\varepsilon=0.3$ (C),(D) as (A),(B) but for $\varepsilon=0.6$ (other parameters: $N_{E}=8000, N_{I}=2000, K=100, J_{0}=1, \tau_{\mathrm{m}}=10 \mathrm{~ms}, \eta=0.9$, the external currents were set according to the balance equation (2.25) with a target average firing rate $\bar{v}_{\text {bal }}=1 \mathrm{~Hz}$ ). 

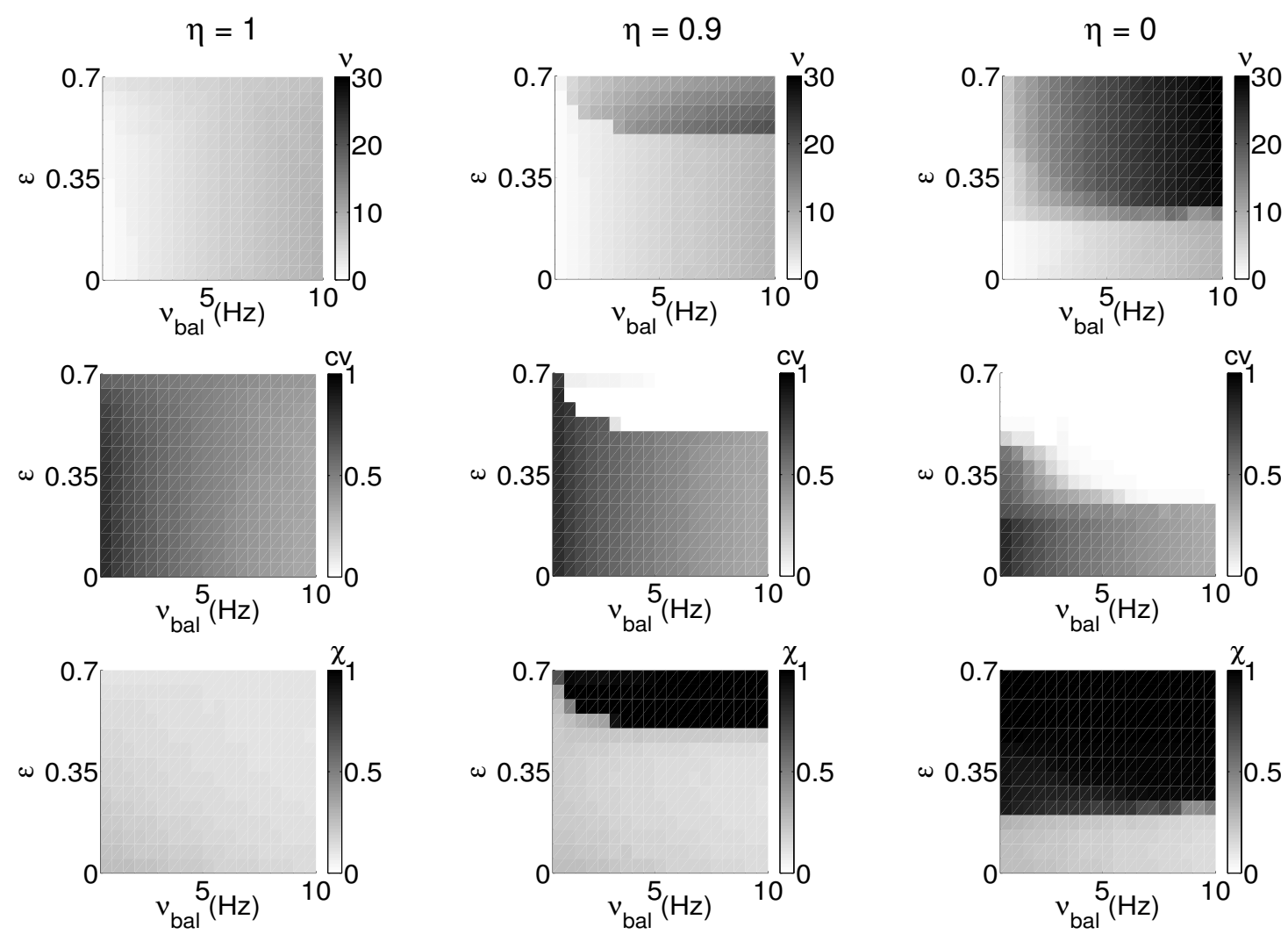

Figure 2.9 - Phase diagrams of excitatory-inhibitory networks for three different ratios $\eta=J_{E E} / J_{I E}$. (see coupling matrix (2.31) ) Columns from left to right: $\eta=1,0.9,0$, from top to bottom: average firing rate $\bar{v}$, coefficient of variation cv and synchrony measure $\chi$; on the $\mathrm{x}$-axis is plotted the target average firing rate according to the balance equation (2.25) and on the y-axis the excitatory-inhibitory feedback loop activation $\varepsilon$, (parameters: $N_{E}=8000, N_{I}=2000, K=100, J_{0}=1, \tau_{\mathrm{m}}=10 \mathrm{~ms}$; displayed are averages of 10 runs with different network realizations).

is conventionally chaotic, indicated by positive and finite largest Lyapunov exponents (Fig. 2.10C). Interestingly, the Lyapunov spectra were invariant to the network size. Plotting the Lyapunov exponents $\left\{\lambda_{i}\right\}$ versus their indices $i$ rescaled with the network size $N$, they converged to a unique shape for large $N$ (Fig. 2.10 C). Consequently, the number of positive Lyapunov exponents, the entropy production rate $H, \mathrm{Eq}$. (1.5), and the attractor dimension $D$, Eq. (1.6), increased linearly with $N$ (Fig. 2.10D,E). This indicates extensive chaos and it is well justified to define the relative attractor dimension $d=D / N$ and the average entropy production rate per neuron $h=H / N$ (Fig. 2.10F,G). Both quantities were surprisingly high, symbolizing a high dimensional chaotic attractor in these networks and a rapid entropy production. To show that extensive chaos is a robust phenomenon in the studied networks, additional network size invariant Lyapunov spectra for different average firing rates $\bar{v}$ are presented in Fig. $2.10 \mathrm{H}-\mathrm{K}$.

Increasing the network-averaged firing rate $\bar{v}$ intensified the chaos in the balanced networks (Fig. 2.11). Although the irregularity in the neurons' spiking activity decreased, the largest Lyapunov exponent, the number of positive Lyapunov exponents, attractor dimension and entropy production rate increased with increasing firing rate. We plotted the entropy production rate divided by the average firing rate in Fig. $2.11 \mathrm{G}$, providing an estimate of the average entropy production per spike per neuron. The surprisingly high rate of entropy production of up to 1 bit per spike per neuron should be contrasted with the actual information content in cortical neurons supplied by 

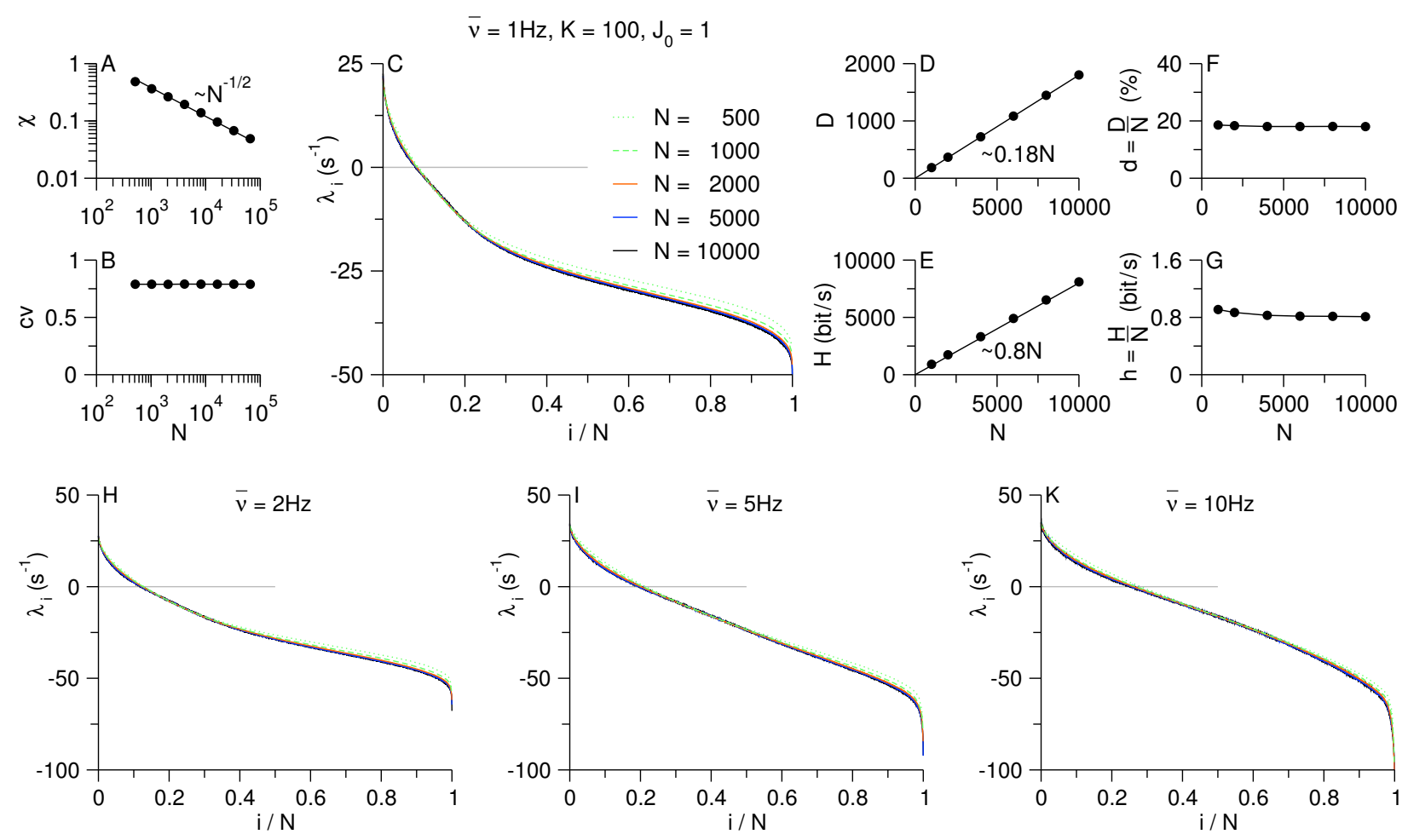

Figure 2.10 - Extensive chaos in balanced inhibitory networks of $N$ theta neurons. (A) Synchrony measure $\chi$ (straight line: $\chi \sim 1 / \sqrt{N}$ ), (B) average coefficient of variation $\mathrm{cv}$, (C) full Lyapunov spectra $\left\{\lambda_{i}\right\}$, (D) attractor dimension $D$, Eq. (1.6), (straight line: $D=0.18 N$ ), (E) entropy production rate $H$, Eq. (1.5), (straight line: $H=0.8 N$ ), (F) attractor dimension in percent of phase space dimension $d=D / N$ and $(\mathrm{G})$ entropy production rate per neuron $h=H / N$, (H)-(K) full Lyapunov spectra for different firing rates $\bar{v},\left(\tau_{\mathrm{m}}=10 \mathrm{~ms}\right.$; displayed are averages of 10 runs with different network realizations $)$.
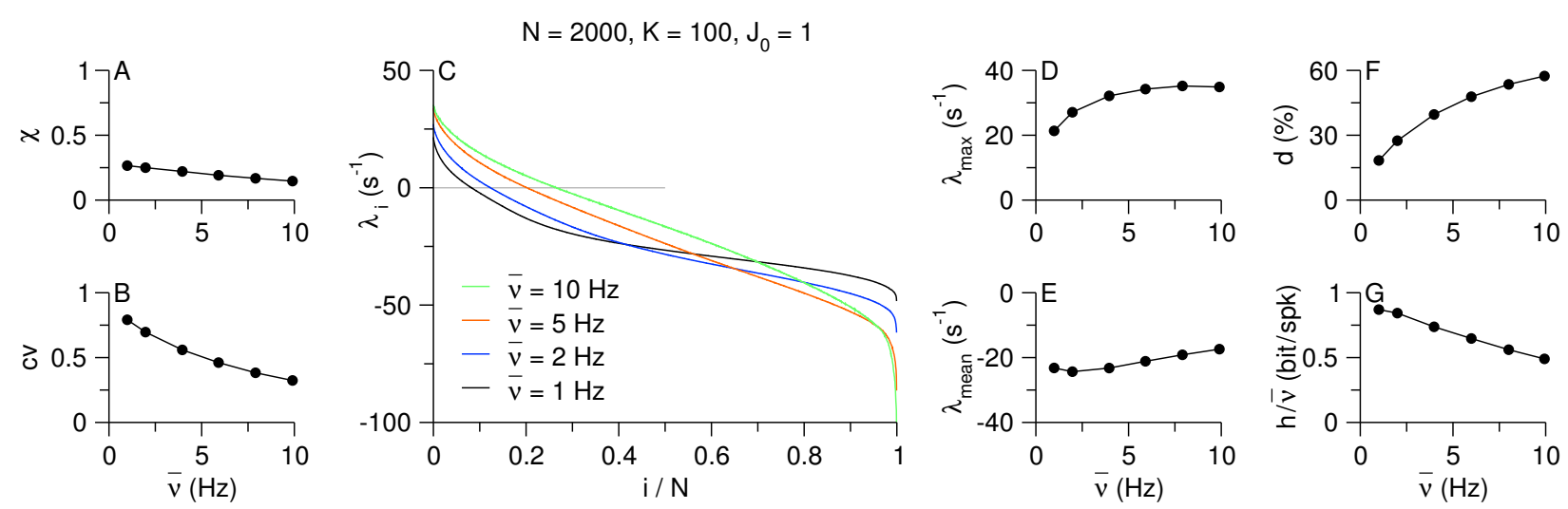

Figure 2.11 - Deterministic chaos in balanced inhibitory networks for varied average firing rates $\bar{v}$. (A) Synchrony measure $\chi$, (B) average coefficient of variation cv, (C) full Lyapunov spectra $\left\{\lambda_{i}\right\}$, (D) largest Lyapunov exponent $\lambda_{\max }=\lambda_{1}$, (E) mean Lyapunov exponent $\lambda_{\text {mean }}=\frac{1}{N} \sum_{i} \lambda_{i},(\mathrm{~F})$ attractor dimension in percent of phase space dimension $d=D / N$ and $(\mathrm{G})$ entropy production rate per neuron $h=H / N,\left(\tau_{\mathrm{m}}=\right.$ $10 \mathrm{~ms}$; displayed are averages of 10 runs with different network realizations). 

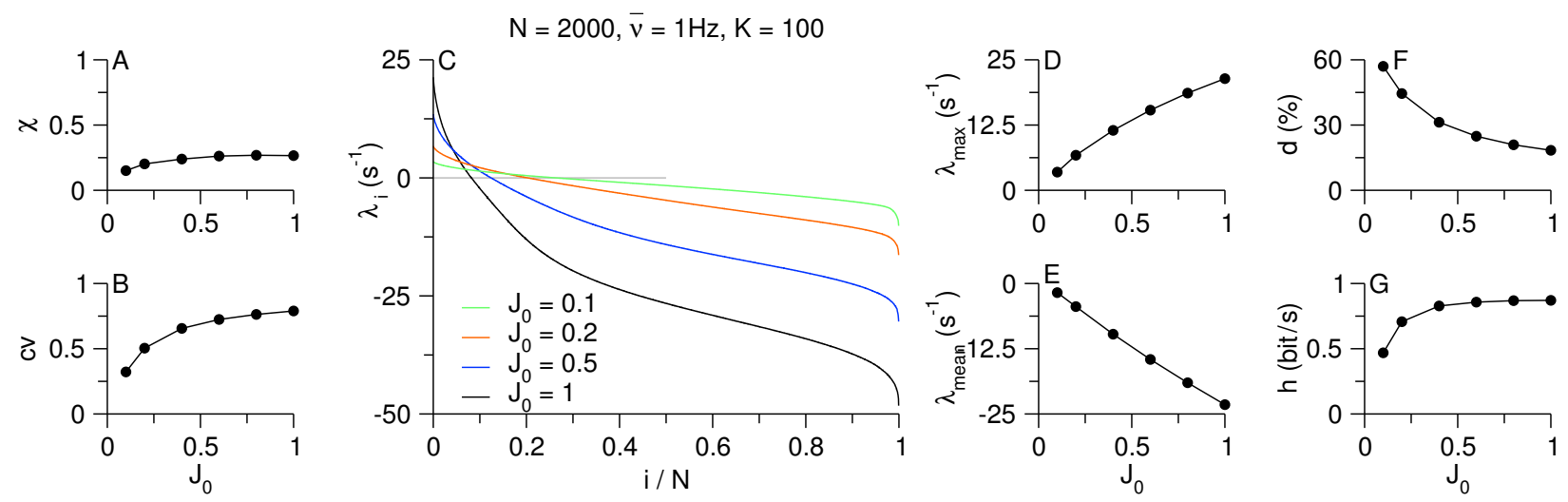

Figure 2.12 - Deterministic chaos in balanced inhibitory networks for varied coupling strengths $J_{0}$. (see Fig. 2.11 for description)
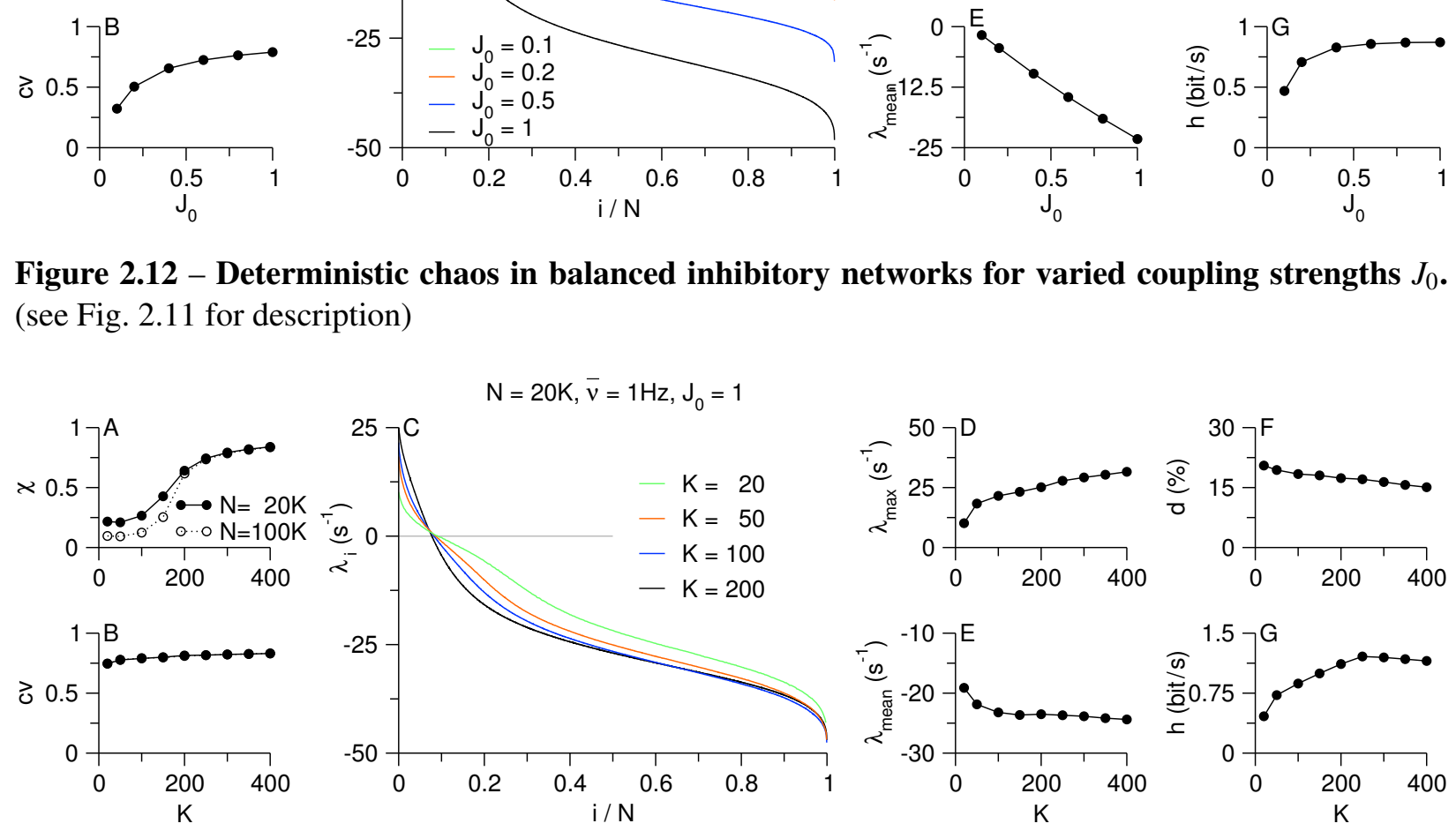

Figure 2.13 - Deterministic chaos in balanced inhibitory networks for varied number of presynaptic neurons $K$. A transition from the asynchronous irregular balance state to a synchronous irregular state occurs at a network size independent $K_{c} \approx 200$ (see Fig. 2.11 for description).

sensory input streams. This was experimentally estimated to be of order 1 bit per spike per neuron as well [93, 94]. Our results thus imply a rapid loss of input information in such chaotic networks.

Does the result of a rapid information loss strongly depend on other parameters in our model? The membrane time constant $\tau_{\mathrm{m}}$ is set to $10 \mathrm{~ms}$ throughout this paper, which is the physiological relevant time scale for membrane time constants. Since it is 'just' the unit of any time-related quantity in our model, changing $\tau_{\mathrm{m}}$ would just change the time-related quantities accordingly. For example, doubling $\tau_{\mathrm{m}}$ to $20 \mathrm{~ms}$, would result in half the average firing rates and Lyapunov exponents in Fig. 2.11. The attractor dimension and the average entropy production rate per spike, however, would not be affected by a change of the membrane time constant.

The influence of the coupling strength $J_{0}$ is displayed in Fig. 2.12. A vanishing $J_{0}$ would correspond to the uncoupled case, in which these fully deterministic systems would exhibit stable regular dynamics. Increasing $J_{0}$ led to the asynchronous, irregular, chaotic balanced state. While the quantitative values of the Lyapunov exponents depended on the coupling strength, the synchrony measure, coefficient of variation, attractor dimension and entropy production rate appeared to saturate at $J_{0}$ of the order of one.

Surprisingly, a large number of synapses per neuron $K$ led to a transition from the asynchronous chaotic state to a synchronous chaotic state in inhibitory networks (Fig. 2.13). This behavior was rather unexpected, as the statistics of the balanced state should be independent of $K$ in the large 

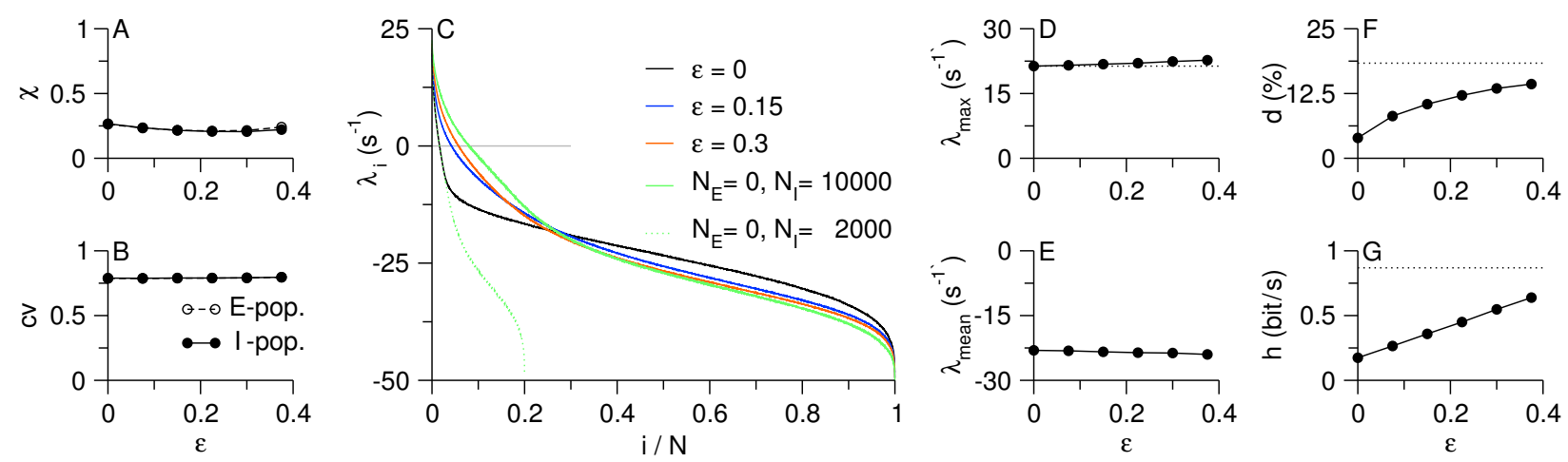

Figure 2.14 - Chaotic dynamics in balanced excitatory-inhibitory networks while activating the excitatory-inhibitory feedback loops with $\varepsilon$. (values of inhibitory networks as dotted lines for comparison) (A) Synchrony measure $\chi$, (B) average coefficient of variation cv, (C) full Lyapunov spectra $\left\{\lambda_{i}\right\}$, (D) largest Lyapunov exponent $\lambda_{\max }=\lambda_{1}$, (E) mean Lyapunov exponent $\lambda_{\text {mean }}=\frac{1}{N} \sum_{i} \lambda_{i},(\mathrm{~F})$ attractor dimension in percent of phase space dimension $d=D / N$ and (G) entropy production rate per neuron $h=H / N$, (parameters: $N_{I}=2000, N_{E}=8000, K=100, \bar{v}_{E}=\bar{v}_{I}=1 \mathrm{~Hz}, J_{0}=1, \tau_{\mathrm{m}}=10 \mathrm{~ms}, \eta=0.9, \varepsilon=0.3$; displayed are averages of 10 runs, with different network realizations, in (C) averages of 3 runs).

$K$-limit, due to the specific square root scaling of the external currents and coupling strengths. However, increasing $K$ while assuring the same connection probability between neurons $p=K / N$, destabilized the asynchronous state. The occurrence of this transition at a critical connectivity $K_{c} \approx$ 200 that was independent of $N$ as indicated by the results shown in Fig. 2.13A for $p=0.05$ (filled circles) and $p=0.01$ (open circles). The asynchrony for $K<200$ was confirmed by a decreasing synchrony measure $\chi$ for increasing $N$. Such a transition, with a network size independent critical connectivity was previously reported in networks of inhibitory hippocampal interneurons [38]. One should note that the irregularity of the individual spike trains was insensitive to the transition. The asynchronous and the synchronous state displayed identical, high coefficients of variation. The collective dynamics of the inhibitory networks were only slightly influenced by the transition from asynchrony to synchrony as well. In the asynchronous state, the largest Lyapunov exponent and the entropy production rate slowly increased, the attractor dimension slightly decreased with increasing $K$. In the synchronous state, the largest Lyapunov exponent continued to increase and the information dimension continued to decrease, whereas the entropy production rate changed to slowly decrease with increasing $K$.

\subsection{Dynamics of Excitatory-Inhibitory Networks}

Realistic cortical networks typically consist of $20 \%$ inhibitory and $80 \%$ excitatory neurons [95]. We therefore investigated the influence of excitatory coupling on the dynamics of the balanced state (Fig. 2.14). For a quantitative comparison with inhibitory networks, the coupling strengths were chosen as explained above, Eq. 2.31,

$$
\mathbf{J}=\frac{J_{0}}{\sqrt{K}}\left(\begin{array}{cc}
0.9 \varepsilon & -\sqrt{1-(0.9 \varepsilon)^{2}} \\
\varepsilon & -\sqrt{1-\varepsilon^{2}}
\end{array}\right) .
$$

With this parametrization, the magnitude of input current fluctuations remained unchanged while increasing the strength of the excitatory-inhibitory feedback loops with $\varepsilon$. Thus the firing statistics 

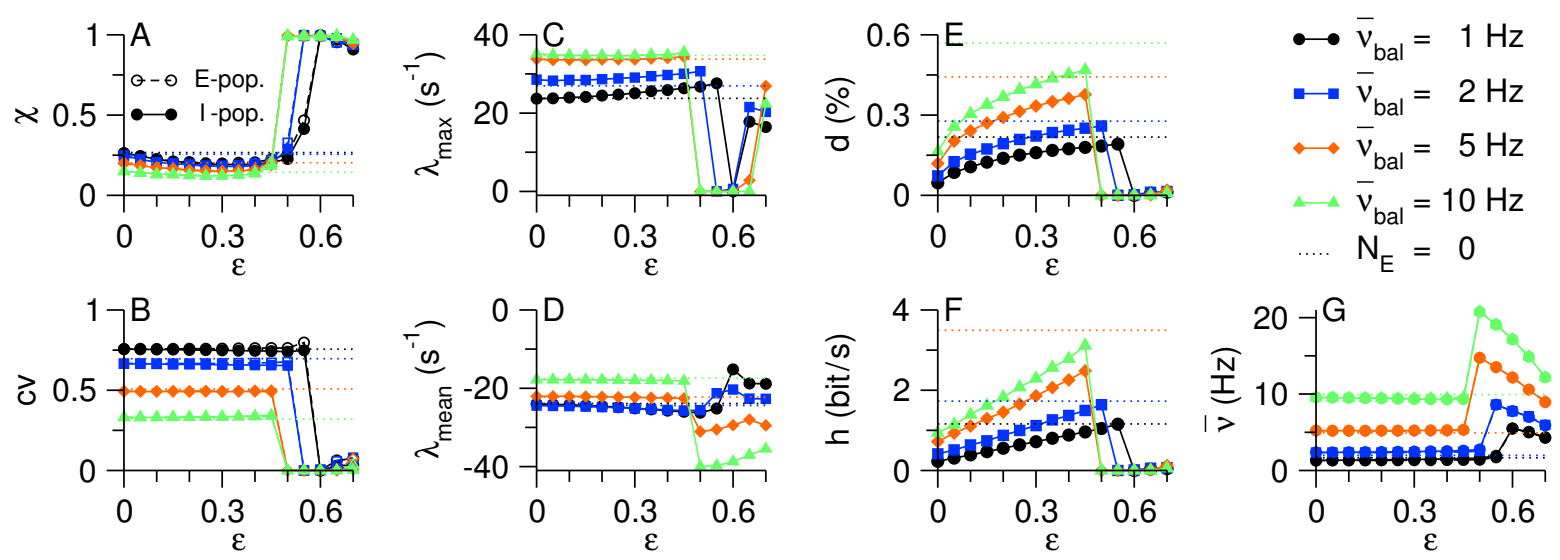

Figure 2.15 - Stable dynamics during transition from asynchronous irregular to synchronous regular state for strong excitatory couplings $\varepsilon$. (values of inhibitory networks as dotted lines for comparison) (A) Synchrony measure $\chi$, (B) average coefficient of variation $\mathrm{cv}$, (C) largest Lyapunov exponent, (D) mean Lyapunov exponent, (E) attractor dimension in percent of phase space dimension, $(\mathrm{F})$ entropy production rate per neuron, $(\mathrm{G})$ actual population-averaged firing rates, (parameters: $N_{I}=2000, N_{E}=8000, K=100$, $J_{0}=1, \tau_{\mathrm{m}}=10 \mathrm{~ms}, \eta=0.9$, the input currents were here chosen to fulfill the balance equation (2.25) for a certain target average firing rate $\bar{v}_{\text {bal }}$; displayed are averages of 3 runs with different network realizations).

remained unchanged while increasing $\varepsilon$ (Fig. 2.14A,B). For $\varepsilon=0$, all excitatory neurons were passive, in the sense that they did not provide any feedback to the network and their dynamics was dominated by the inputs from the inhibitory neurons. The Lyapunov spectrum was therefore very similar to an equivalent spectrum of a network of exclusively inhibitory neurons of one fifth size (dotted line in Fig. 2.14C). In fact, the positive part was identical, which suggests that the unstable modes were due to the active inhibitory neurons. Consequently, the largest Lyapunov exponent was identical to the value of the inhibitory networks (Fig. 2.14D), while the attractor dimension and entropy production rate at $\varepsilon=0$ were one fifth of the inhibitory values (Fig. 2.14F,G). Upon activation of the excitatory-inhibitory feedback loops with $\varepsilon>0$, the chaos in the networks increased. Although the largest Lyapunov exponent hardly increased in magnitude, the attractor dimension and entropy production showed a strong dependence on the excitatory coupling. The increasing attractor dimension seemed to saturate below the inhibitory value, while the entropy production increased linearly, but did not exceed the values of inhibitory networks (dotted lines), because the aforementioned transition to the synchronous state occurred beforehand. It is thus interesting to see, how this is affected by the transition to the synchronous state.

During the transition from the asynchronous irregular state to a synchronous regular state in excitatory-inhibitory networks the firing statistics and the collective dynamics changed considerably (Fig. 2.15). The sudden change to $\chi=1$ and $\mathrm{cv}=0$ indicate the synchronous and regular firing activity above $\varepsilon_{c}$, whose precise value depended on the average firing rate $\bar{v}$. During the transition to the synchronous state, the actual population-averaged firing rates increased abruptly, and strongly deviated from the expected firing rates $\bar{v}_{\text {bal }}$ from the balance equation (2.25). The largest Lyapunov exponent and entropy production rate vanished, and the attractor dimension became one. This indicates stable periodic dynamics during this transition. Note, that the entropy production rate and attractor dimension never increased the values of inhibitory networks (dotted lines). We will now continue with the description of the dynamics of the asynchronous irregular balanced state.

Increasing the number of excitatory neurons in networks with a fixed number of inhibitory neu- 

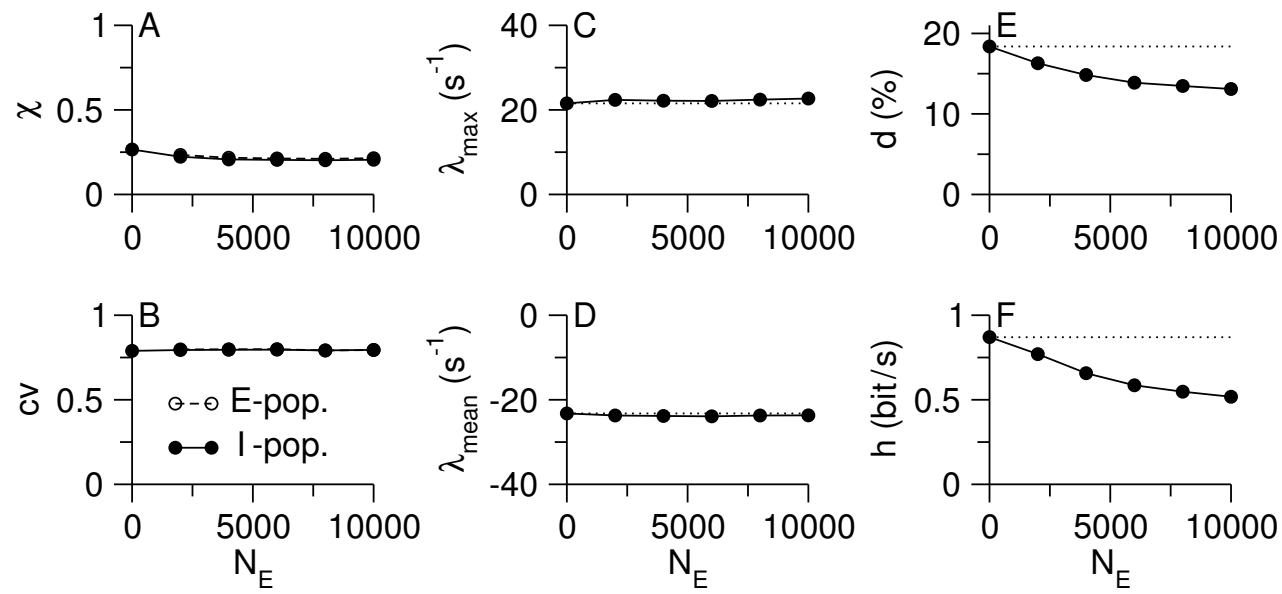

Figure 2.16 - Chaotic dynamics in balanced excitatory-inhibitory networks for varied number of excitatory neurons $N_{E}$. (values of inhibitory networks as dotted lines for comparison) (A) Synchrony measure $\chi$, (B) average coefficient of variation cv, (C) largest Lyapunov exponent, (D) mean Lyapunov exponent, (E) attractor dimension in percent of phase space dimension and (F) entropy production rate per neuron, (parameters: $N_{I}=2000, N_{E}=8000, K=100, \bar{v}_{E}=\bar{v}_{I}=1 \mathrm{~Hz}, J_{0}=1, \tau_{\mathrm{m}}=10 \mathrm{~ms}, \eta=0.9, \varepsilon=0.3$; displayed are averages of 10 runs with different network realizations).

rons $N_{I}=2000$ reduced the intensity of the chaos (Fig. 2.16). We increased the number of excitatory neurons from $N_{E}=0$ to $N_{E}=10000$. The excitatory-inhibitory feedback loop strength was set to $\varepsilon=0.3$. Again, the spike statistics did not change, while the dynamics changed in a way that the attractor dimension and entropy production decreased with increasing number of excitatory neurons. The largest Lyapunov exponent was rather unaffected. The high ratio of excitatory neurons in realistic cortical networks might thus be beneficial in terms of an reduced entropy production and hence a slower loss of input information.

The variation of the average firing rate revealed a qualitatively similar dependence of the dynamics in excitatory-inhibitory networks as observed in inhibitory networks (Fig. 2.17). Varying the population-averaged firing rates $\bar{v}_{E}=\bar{v}_{I}=\bar{v}$ did not change the firing statistics in excitatoryinhibitory networks compared to the inhibitory networks. This was expected from the construction of the coupling matrix that led to the same input current statistics in the balanced state in both types of networks. The largest and the mean Lyapunov exponent were also independent of the type of network. Nevertheless, the Lyapunov spectra changed slightly in excitatory-inhibitory networks, such that the attractor dimension and entropy production rate were slightly reduced as discussed above, but showed qualitatively the same dependence on the average firing rate.

Increasing the number of incoming connections per neuron $K$ led to similar dynamics in both types of networks as well, although the transition to the synchronous irregular state occurred at much higher $K_{c}$ in excitatory-inhibitory networks (Fig. 2.18). We checked two ratios $N_{I}=20 \mathrm{~K}$ (filled circles) and $N_{E}=20 \mathrm{~K}$ (open squares), while $80 \%$ of the neurons were excitatory and $20 \%$ were inhibitory $\left(N_{E}=4 N_{I}\right)$ in both cases. The synchronous irregular state emerged for $K>K_{c} \approx 1000$, which is a five times higher critical connectivity compared to exclusively inhibitory networks. In the asynchronous irregular state, the firing statistics, Lyapunov exponents, attractor dimension and entropy production rate were merely independent of the number of synapses $K$. This was expected since the statistics of the balanced state is independent of $K$.

Investigating the dependence of the network dynamics on the number of neurons $N$, we observed extensive chaos in excitatory-inhibitory networks (Fig. 2.19). The firing statistics, captured in the 

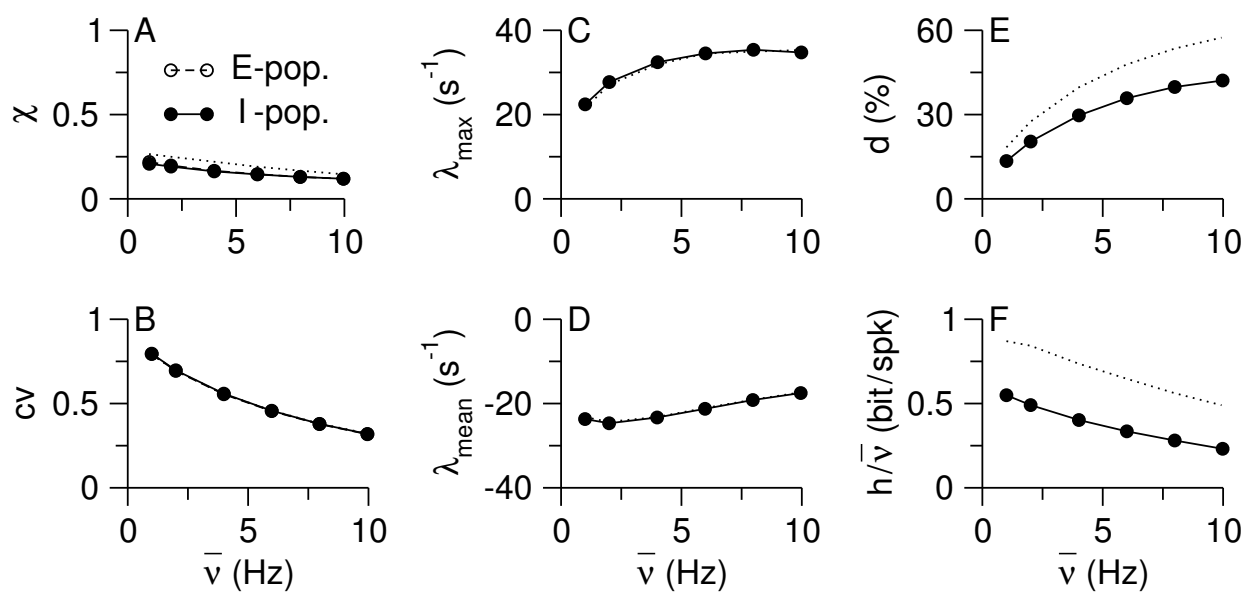

Figure 2.17 - Chaotic dynamics in balanced excitatory-inhibitory networks for varied populationaveraged firing rates $\bar{v}_{E}=\bar{v}_{I}=\bar{v}$. (see Fig. 2.16 for description).

decreasing synchrony measure $\chi \sim 1 / \sqrt{N}$ and constant high coefficients of variation $\mathrm{cv}$, indicate the typical asynchronous irregular balanced state. The Lyapunov spectra converged to a system size independent shape. Hence the number of positive Lyapunov exponents, attractor dimension and entropy production rate scaled linearly with the number of neurons. This suggests a well-defined thermodynamic limit of the dynamics of excitatory-inhibitory networks in the balanced state and confirms the results from inhibitory networks. Altogether, one can conclude that inhibitory networks of theta neurons already captured the dynamics of excitatory-inhibitory networks very well. Both types of networks exhibit extensive deterministic chaos in the balanced state.

\subsection{Temporal Network Chaos}

Are all neurons involved equally and at all times in the chaotic dynamics despite their substantial firing heterogeneities? To answer this question, we studied the first Lyapunov vector $\vec{v}(t)$ (with $\left.\sum_{i=1}^{N} v_{i}(t)^{2}=1\right)$. At each point in time, $\vec{v}$ aligns with the direction in which initial perturbations exponentially grow with maximal asymptotic rate $\lambda_{\max }$. The Lyapunov vector's elements can be assigned to the neurons in the networks. Therefore, they can be used to investigate which neurons were the most sensitive to perturbations. To understand when the neurons were the most sensitive to state perturbations, we examined the voltage distribution of neurons when exhibiting the largest Lyapunov vector element. This distribution compared to the stationary distribution was shifted towards the spike generating instability, marking it as an important source of instability of the collective dynamics (Fig. 2.20 C and Fig. 2.21F,G).

The Lyapunov vector in both types of networks was dominated by relatively small subsets of neurons that changed strongly over time (Fig. 2.20, 2.21A). We analyzed the composition of these groups with two quantities: the participation ratio quantifying the size of a group at a certain time, and the chaos index quantifying the time-averaged participation of individual neurons.

The participation of individual neurons in the chaotic dynamics is captured by the chaos index

$$
c_{i}=\sqrt{N\left\langle v_{i}(t)^{2}\right\rangle_{t}}
$$

the root mean square of the Lyapunov vector elements. If a neuron never participates in the chaotic 

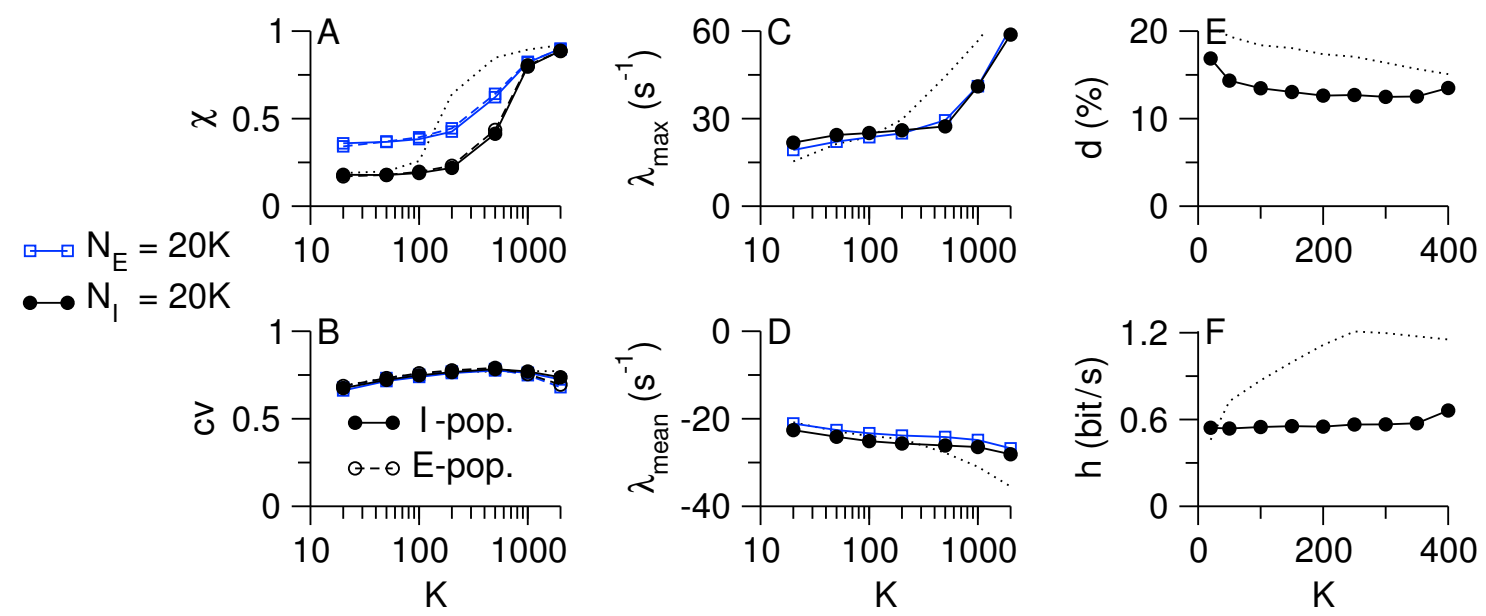

Figure 2.18 - Chaotic dynamics in balanced excitatory-inhibitory networks for varied number of incoming connections $K$. (note the logarithmic axes in (A)-(D), circles: $N_{I}=20 \mathrm{~K}$ and squares: $N_{E}=20 \mathrm{~K}$, $N_{E}=4 N_{I}$; dotted lines: inhibitory networks with $N=20 K$; see Fig. 2.16 for description, in (E), F) averages of 3 runs for $K>200$ ).
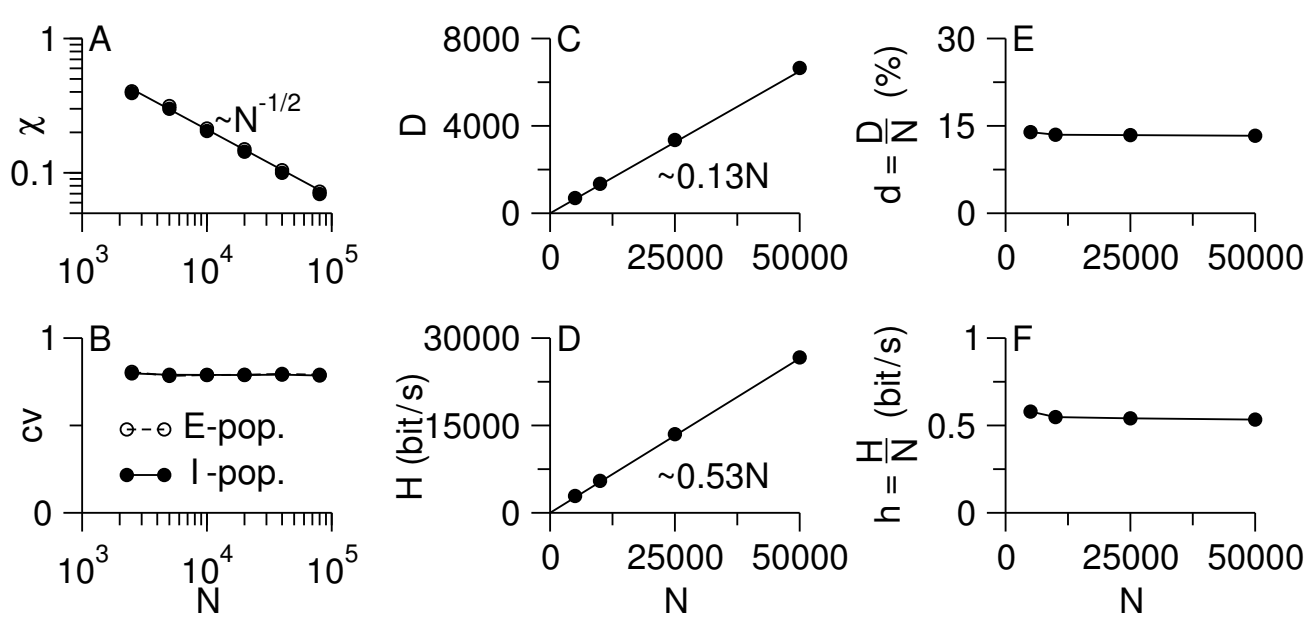

Figure 2.19 - Extensive chaos in balanced excitatory-inhibitory networks for varied network sizes $N$. (A) Synchrony measure $\chi$ (straight line: $\chi \sim 1 / \sqrt{N}$ ), (B) average coefficient of variation cv, (C) Attractor dimension $D$ (straight line: $D=0.13 N$ ), (D) entropy production rate $H$ (straight line: $H=0.53 N)$, (E) information dimension in percent of phase space dimension $d=D / N$ and (F) entropy production rate per neuron $h=H / N,\left(N_{E}=4 N_{I}\right.$ and $N=N_{E}+N_{I}$, other parameters as in Fig. 2.16, displayed are averages of 10 runs with different network realizations). 

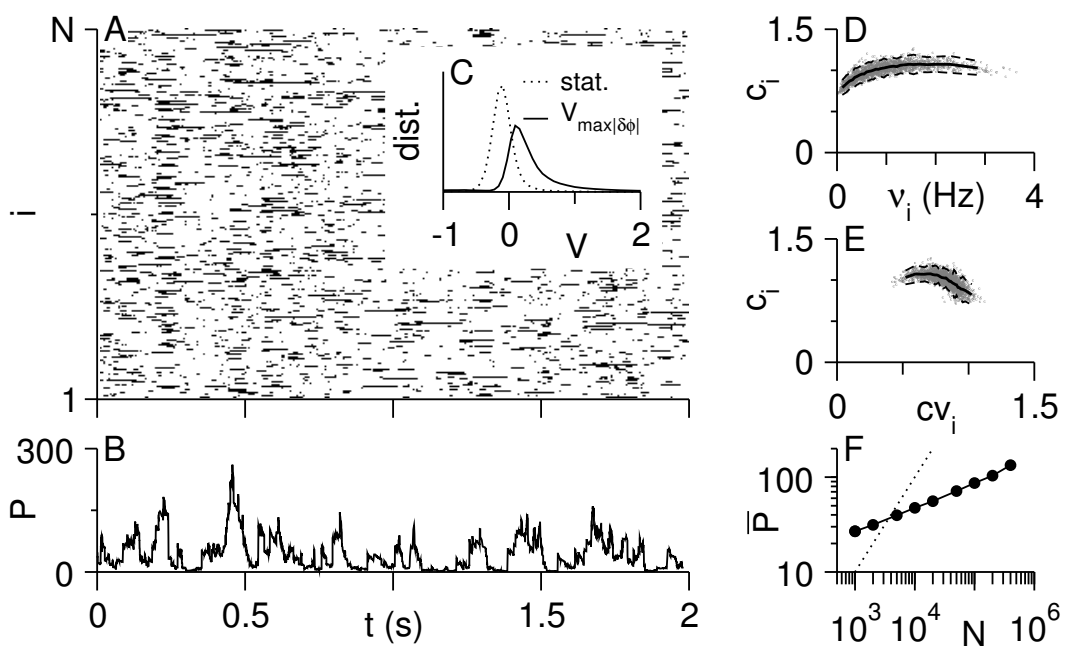

Figure 2.20 - Temporal network chaos in inhibitory balanced networks. (A) First Lyapunov vector $\vec{v}(t)$ (marked black if $\left|v_{i}(t)\right|>1 / \sqrt{N}$ ), (B) Participation ratio $P(\mathrm{t})$ of first Lyapunov vector, (C) Voltage distribution of neurons with largest Lyapunov vector elements (dotted line: stationary distribution) (D,E) Neurons' chaos indices $c_{i}$ versus firing rates $v_{i}$ and coefficients of variation $c v_{i}$ (straight line: mean, dashed lines: \pm 2 std), (F) Average participation ratio $\bar{P}$ vs. network size $N$ (dotted line: guide for the eye for $\bar{P} \sim N$ ), (parameters: $N=2000, K=100, \bar{v}=1 \mathrm{~Hz}, J_{0}=1, \tau_{\mathrm{m}}=10 \mathrm{~ms}$ ).
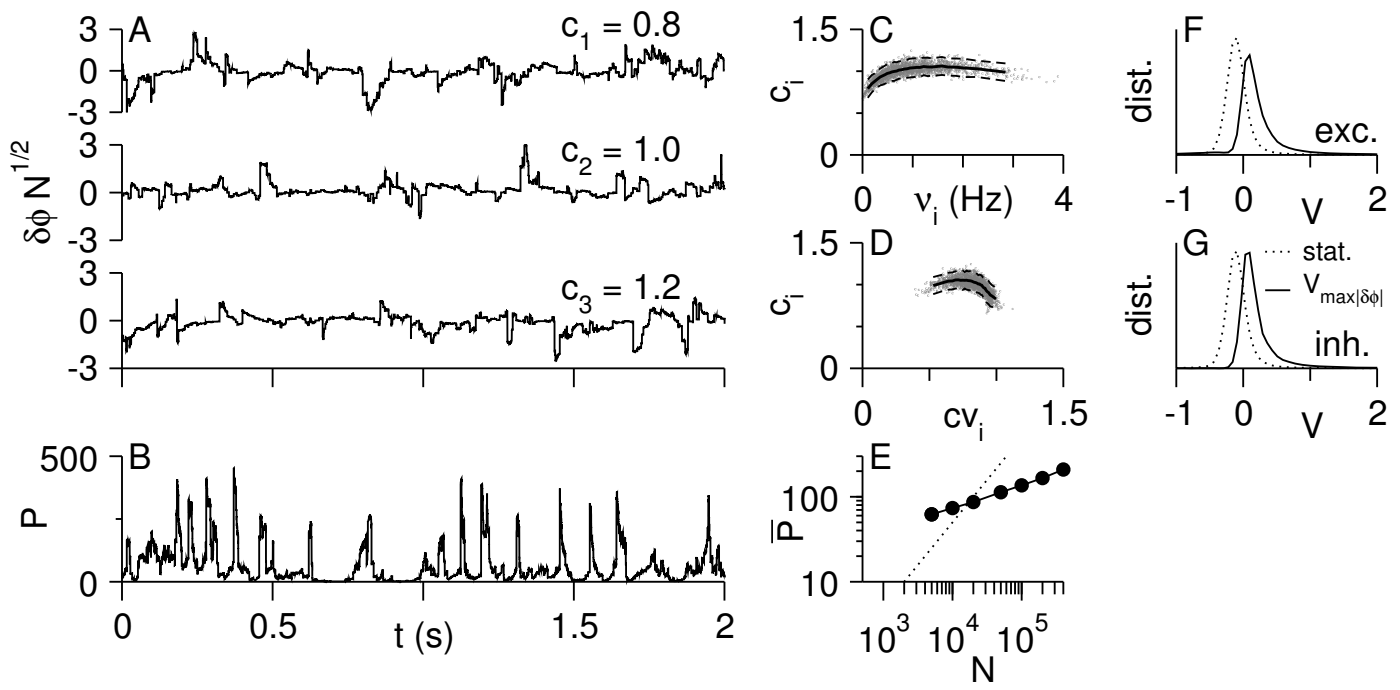

Figure 2.21 - Temporal network chaos in excitatory-inhibitory balanced networks. (A) Lyapunov vector elements $v_{i} \sqrt{N}$ versus time for three neurons with different chaos index $c_{i}$, (B) Participation ratio $P$ versus time, (C) Neurons' chaos indices $c_{i}$ versus their firing rates $v_{i}$ and (D) versus coefficients of variation $c v_{i}$ (subsampled: every 5th dot shown, straight lines: averages, dashed lines: $\pm 2 \mathrm{STD}$ ), (E) Average participation ratio $\bar{P}$ versus network size $N$ (dotted line: guide for the eye for $\bar{P} \sim N$ ), (F) Voltage distribution of excitatory neurons when exhibiting the largest Lyapunov vector element (dotted line: stationary distribution), (G) as (F) for inhibitory neurons, (parameters: $N_{\mathrm{I}}=2000, N_{\mathrm{E}}=8000, K=100, \bar{v}=1 \mathrm{~Hz}, J_{0}=1$, $\tau_{\mathrm{m}}=10 \mathrm{~ms}, \eta=0.9, \varepsilon=0.3$ ). 

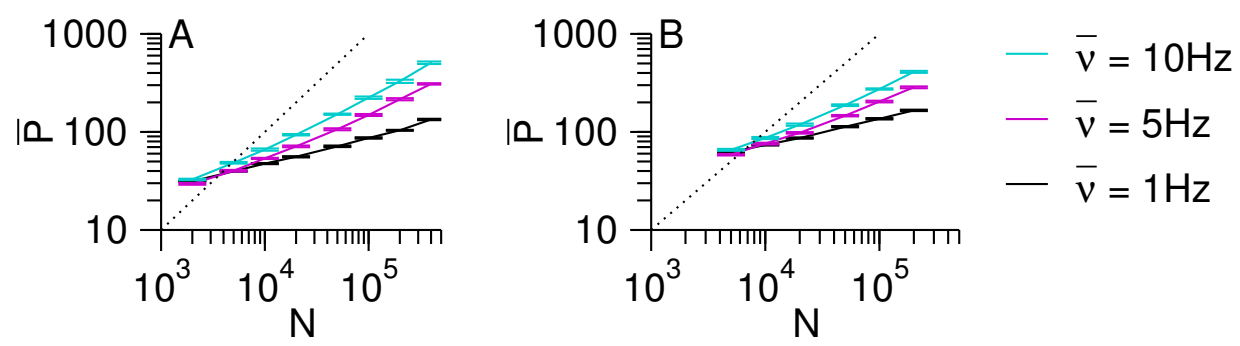

Figure 2.22 - Average participation ratio $\bar{P}$ versus network size $N$ in balanced theta neuron networks with different average firing rates $\bar{v}$. (A) Inhibitory networks (B) Excitatory-inhibitory networks, (dotted line: guide to the eye for $\bar{P} \sim N$ ), (parameters: $N_{\mathrm{I}}=0.2 N, N_{\mathrm{E}}=0.8 N, K=100, J_{0}=1, \tau_{\mathrm{m}}=10 \mathrm{~ms}$, $\varepsilon=0.3)$.

dynamics $\left(v_{i}(t) \equiv 0\right)$, its chaos index is $c_{i}=0$. If only one neuron participates $\left(v_{i}(t) \equiv 1\right)$, only its chaos index does not vanish and is $c_{i}=\sqrt{N}$. If all neurons contribute equally to the chaotic dynamics $\left(v_{i}(t) \approx 1 / \sqrt{N}\right)$, all chaos indices are $c_{i} \approx 1$. Although neurons with low firing rates and high variability had slightly reduced chaos indices, all neurons had a similar chaos index of approximately one (Fig. 2.20, 2.21D,E). This indicates that all neurons participated almost equally in the chaotic dynamics.

The number of neurons participating in the chaotic dynamics at any single point in time is captured by the participation ratio

$$
P(t)=1 / \sum_{i=1}^{N} v_{i}(t)^{4} .
$$

Two limiting cases are interesting for the understanding of the participation ratio: (i) Delocalized (extended) states, where the Lyapunov vector is spread out over the entire network. Each vector element is then $v_{i}(t) \approx 1 / \sqrt{N}$ and the participation ratio $P(t)=1 /\left(N / N^{2}\right)=N$. (ii) Localized states, where the Lyapunov vector is localized to a few neurons. Then, only a few vector elements do not vanish and the participation ratio is $P(t) \ll N$. The participation ratio exhibited substantial fluctuations indicating strongly varying group sizes (Fig. 2.20, 2.21B). For the considered rates $1 \mathrm{~Hz} \leq \bar{v} \leq 10 \mathrm{~Hz}$, the time-averaged participation ratio $\bar{P}=\langle P(t)\rangle_{t}$ obeyed a sublinear scaling $\bar{P} \sim N^{\alpha}$, with $0.25 \leq \alpha \leq 0.5$ (Fig. 2.22). This behavior neither clearly indicates localized states, for which $\bar{P}$ would be independent of $N$, nor delocalized states, where $\bar{P}$ would depend linearly on $N$. One should, however, note that the groups are generally small relative to the network sizes, the fraction of most unstable neurons decreased algebraically as $N^{\alpha-1}$.

These results of the chaos index and the participation ratio can be summarized in the following. Although at one point in time only a small group of neurons participated in the chaotic dynamics, the neurons composing these groups constantly changed over time such that in the long run all neurons participated almost equally in the chaotic dynamics.

\subsection{Covariant Lyapunov Vectors and Hyperbolicity}

While the largest Lyapunov vector yielded interesting results about the active participation of the neurons in the chaotic dynamics, the $N-1$ other Lyapunov vectors yield additional insight into the collective dynamics of neural networks. The standard algorithm for the computation of the Lyapunov spectra evolves an orthonormal system (ONS) with the single spike Jacobians. The norms of the evolved ONS asymptotically yield the Lyapunov spectrum. The ONS itself is spanned 
by vectors of the subspaces $E_{i}$ related to the Lyapunov exponents $\lambda_{<i}$ (see section 2.3.2).

The eigenvectors of the Oseledec matrix that span the subspaces $E_{i}$ related to the Lyapunov exponents are the covariant Lyapunov vectors (CLVs). Only the first vector of the ONS is equivalent with the first CLV. All other vectors in the ONS are not the CLVs-the corresponding eigenvectors to the exponents. Interestingly the CLVs are accessible from the information contained in the ONS. A procedure to obtained the CLVs from the ONS was introduced in Ref. [96]. This vastly extends the approach for the characterization of the dynamics of spiking neuron networks.

Here, we discuss the hyperbolicity of the studied systems to validate the use of the Lyapunov exponents for the derivation of the attractor dimension and entropy production rate. A dynamical system is called hyperbolic if the stable and unstable manifolds are everywhere transversal to each other [96]. This will be tested by determining the angles between all CLVs. In case of transversality of the manifolds, thus hyperbolicity of the system, it can be shown that the system is an Axiom A dynamical system and then an SRB measure exists [67]. The existence of an SRB measure is sufficient for the Pesin identity and the Kaplan-Yorke conjecture to hold [72, 73]. Therefore, proving hyperbolicity of the studied systems validates the use of the Pesin identity and the KaplanYorke conjecture to derive the entropy production rate $H$ and the information dimension (attractor dimension) $D$ form the spectrum of Lyapunov exponents. If hyperbolicity is violated then $H$ and $D$ and provide upper bounds to the entropy production rate and information dimension.

The angles of all CLVs in both inhibitory and excitatory-inhibitory networks are displayed in Fig. 2.23. The computation of the CLVs requires saving the projection matrices obtained in the Gram-Schmidt orthogonalization procedure at all time steps. This restricts the use of the algorithm to moderately large networks up to $N=1000$ so far 1 . As a health check for the computation of the Lyapunov spectra with the ONS and CLVs, respectively, the spectra obtained from both methods are compared in Fig. 2.23A. In inhibitory networks, where longer simulations were possible they show excellent agreement. In excitatory-inhibitory networks, with shorter simulation due to the memory restrictions, there were slight differences. We should therefore focus on the results of the inhibitory networks for which longer and converged calculations can be presented.

At each time step s, the CLVs point in different directions $\left\{\vec{v}_{i, s}\right\}$. The angles between different CLVs $i$ and $j$ are

$$
\alpha_{i j, s}=\arccos \left(\vec{v}_{i, s} \cdot \vec{v}_{j, s}\right) .
$$

We used circular statistics ${ }^{2}$ to calculate their averages $\bar{\alpha}_{i j}$ and variances $\sigma_{i j}^{2}$. The cosine of the average angle $\cos \left(\bar{\alpha}_{i j}\right)$ between all pairs of CLVs is plotted in the lower right triangle and the circular variance $\sigma_{i j}^{2}$ is plotted in the upper left triangle of Fig. $2.23 \mathrm{~B}$. One can see that CLVs with quite differing indices (far away from the diagonal) are orthogonal to each other, the cosine of the average and the variance are zero. CLVs with similar indices (close to the diagonal) have nonvanishing $\cos \left(\bar{\alpha}_{i j}\right)$ and nonvanishing $\sigma_{i j}^{2}$. A closer look at the first offdiagonal reveals that adjacent CLVs can in fact be almost tangential (Fig. 2.23 C). However, it seems that this is the case for larger indices, thus negative Lyapunov exponents. The CLVs corresponding to the positive Lyapunov exponents seem to be transversal. The angles between Lyapunov vectors further apart in their indices become more and more transversal as depicted in Fig. 2.23 C and indicated by the

\footnotetext{
${ }^{1}$ The projection matrix of $N^{2} / 2$ doubles is stored for each of the $\mathscr{O}(10 N)$ time steps. Thus, the necessary memory for these computations quickly reaches the order of gigabytes and hence the limit of available RAM (e.g. $N=1000$ needs $\left.8 \mathrm{~B} \cdot 5 N^{3} \approx 40 \mathrm{~GB} \mathrm{RAM}\right)$. This restricts the method to networks with $N \leq 1000$ so far.

${ }^{2}$ The averages over all considered time steps $s=1 \ldots N_{s}$ called $C_{i j}=\frac{1}{N_{s}} \sum_{s} \cos \alpha_{i j}$ and $S_{i j}=\frac{1}{N_{s}} \sum_{s} \sin \alpha_{i j}$ lead to the circular average $\bar{\alpha}_{i j}=\arctan \left(S_{i j} / C_{i j}\right)$ and circular variance $\sigma_{i j}^{2}=1-\sqrt{C_{i j}^{2}+S_{i j}^{2}}$.
} 
corresponding histograms in Fig. 2.23D.

Finally, we want to hint at the interesting fact that $\cos \left(\bar{\alpha}_{i j}\right)$ and $\sigma_{i j}^{2}$ both vanish for all CLVs with the CLV corresponding to the near zero Lyapunov exponent $i=43$ (see the white hair line in Fig. 2.23B). Any component of a perturbation in the direction of the trajectory would just correspond to a time-shift and thus have a vanishing Lyapunov exponent. Therefore, all CLVs with nonvanishing Lyapunov exponent must be orthogonal to the CLV with vanishing Lyapunov exponent pointing in the direction of the trajectory. This is reflected in the white hair line in Fig. 2.23B.

The index with the vanishing Lyapunov exponent is also important for the test of hyperbolicity as it separates the stable and unstable manifold. The CLVs with larger indices (negative Lyapunov exponents) span the stable manifold $E_{s}^{+}$, whereas the CLVs with smaller indices (positive Lyapunov exponents) span the unstable manifold $E_{s}^{-}$. The minimal angle between the stable and unstable manifold determines the hyperbolicity of the studied systems. At each time step, this angle $\Phi_{s}$ is obtained from the minimal angle between all pairs of CLVs from the stable and the unstable manifold, the least transversal pair of CLVs [96]:

$$
\Phi_{s}=\min \left\{\alpha_{i j, s} \mid \vec{v}_{i, s} \in E_{s}^{+}, \vec{v}_{j, s} \in E_{s}^{-}\right\}
$$

In Fig. 2.24A are shown the angles $\Phi_{s}$ between the stable and unstable manifold at each time step $s$ (spikes in the networks) for different network sizes $N$. The histogram of these angles shown in Fig. 2.24B indicates some hyperbolicity-violations, especially for small networks with $N=100$. From data in Fig. 2.24 it is difficult to conclude that the higher-dimensional the systems get the fewer hyperbolicity-violations occur. This is, however, generally expected [96] and our simulations do not disprove it. We can thus carefully consider the large networks studied to be hyperbolic systems. Then the Pesin identity for the entropy production rate $H_{K S}=\sum_{\lambda_{i}>0} \lambda_{i}$ and the KaplanYorke conjecture for the information dimension $D_{1}=d+\frac{\sum_{i=1}^{d} \lambda_{i}}{\left|\lambda_{d+1}\right|}$ with $d=\max \left\{n: \sum_{i=1}^{n} \lambda_{i} \geq 0\right\}$ hold, and validate the presented approach to quantify the dynamical entropy production rate in neural networks based on the Lyapunov spectra. 

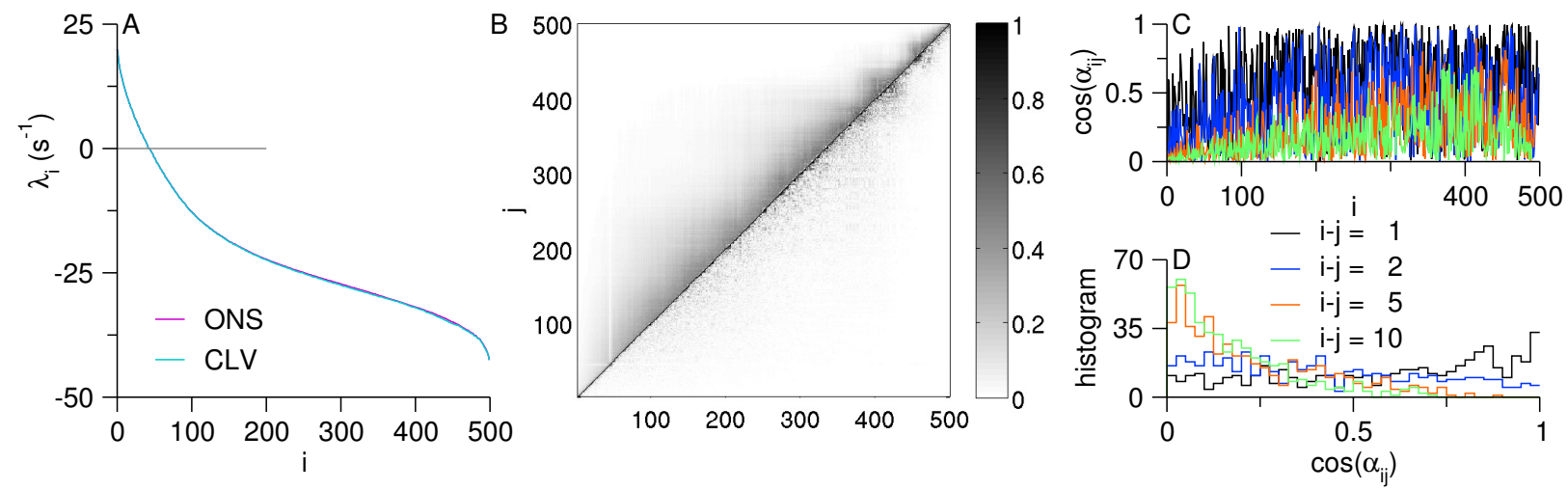

(a) Inhibitory networks $N=500$ (calculation with 25000 spikes).
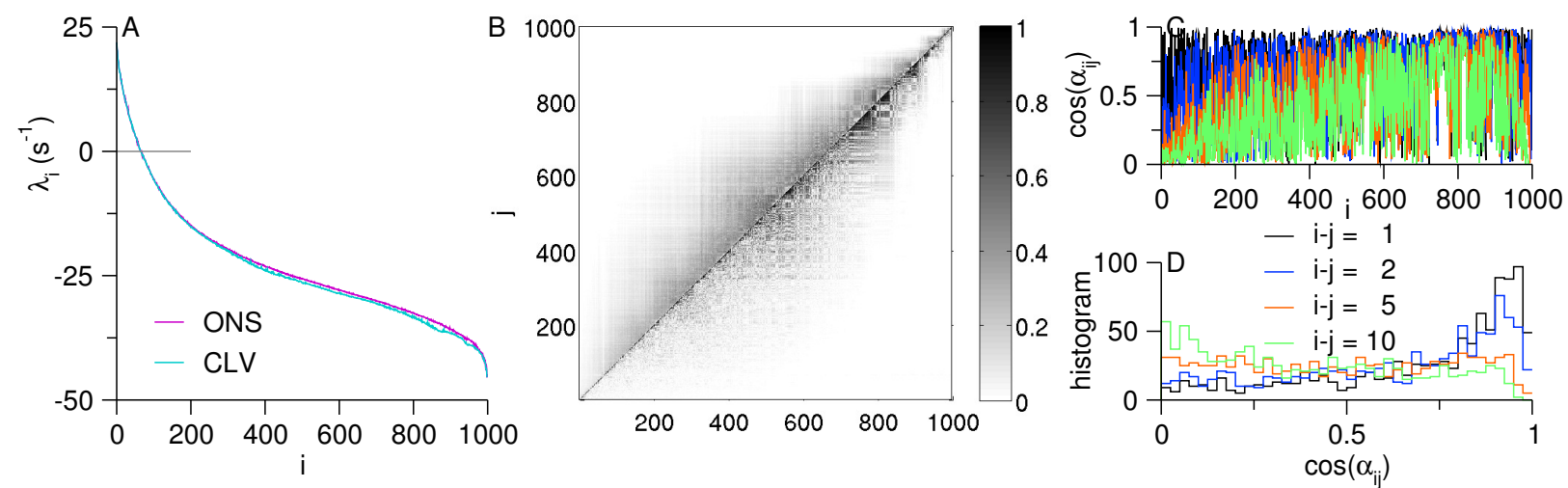

(b) Excitatory-inhibitory networks $N_{E}=500, N_{I}=500$ (calculation with 10000 spikes, $\varepsilon=0.2$ ).

Figure 2.23 - Covariant Lyapunov vectors in balanced theta neuron networks. (A) Lyapunov spectrum obtained in the standard procedure with an orthonormal system (ONS) in the forward Gram-Schmidtreorthogonalization procedure [69] and obtained with the covariant Lyapunov vectors (CLV) from the backward calculation [96], (B) Angles between covariant Lyapunov vectors $\cos \left(\alpha_{i j}\right)=\vec{v}_{i} \cdot \vec{v}_{j}$ (cosine of circular mean $\bar{\alpha}_{i j}$ in lower right triangle, circular variance $\sigma_{i j}^{2}$ in upper left triangle), (C) cosine of the offdiagonal elements $i-j=1,2,5,10$ of the circular mean $\bar{\alpha}_{i j}$, (D) histogram of the offdiagonal $\bar{\alpha}_{i j}$ from (C), (parameters: $\left.\bar{v}=1 \mathrm{~Hz}, K=100, J_{0}=1, \tau_{\mathrm{m}}=10 \mathrm{~ms}\right)$.
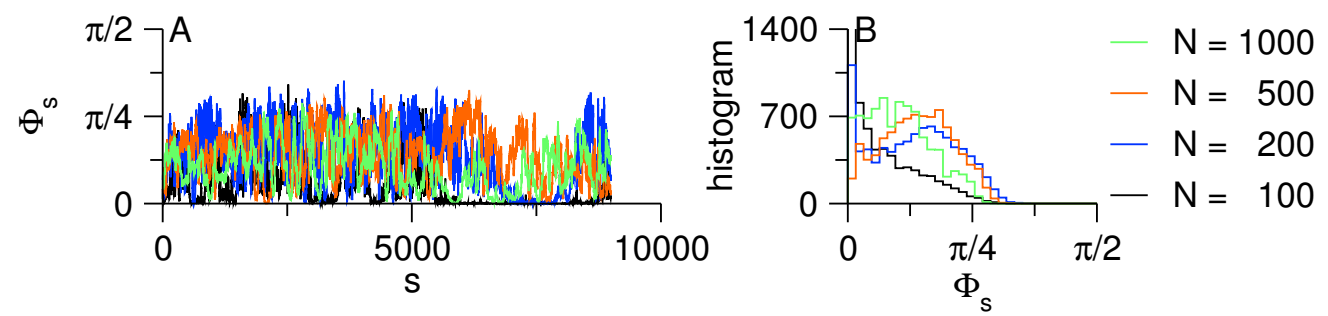

Figure 2.24 - Minimal angle between stable and unstable manifold. (A) Minimal angle $\Phi_{s}$ of the least transversal pair of CLVs versus time step $s$, Eq. (2.54), (B) histogram of points in (A) (parameters: $\bar{v}=1 \mathrm{~Hz}$, $\left.K=50, J_{0}=1, \tau_{\mathrm{m}}=10 \mathrm{~ms}\right)$. 


\subsection{Summary}

In this chapter we introduced a novel approach for a thorough characterization and quantification of the dynamics of spiking neuron networks. This approach allows for the numerically exact simulation of neural network dynamics and the calculation of the complete Lyapunov spectra of networks with arbitrary topologies and single neuron phase-response curves. As a fundamental example, we analyzed networks of theta neurons in the balanced state. The theta neuron model is the canonical form of type I excitable neurons and the balanced state, the prevailing explanation of the asynchronous irregular firing activity observed in the cortex. We therefore expect these results to be representative of a wide class of models of neural networks.

The balanced state emerged for a wide parameter range in both exclusively inhibitorily coupled networks and networks with excitatory and inhibitory populations. We also observed two phase transitions from the asynchronous balanced state to a synchronous state. Beyond a critical connectivity, that was independent of the networks' size, the networks settled into a synchronous irregular state and for very strong excitatory coupling, the networks settled into a synchronous regular state.

The presented results of the collective network dynamics show that theta neurons in the asynchronous irregular balanced state exhibit:

- Deterministic chaos, characterized by positive and finite Lyapunov exponents.

- Extensive chaos, characterized by network size-invariant Lyapunov spectra and a linear increase of the number of positive Lyapunov exponents, attractor dimension and entropy production rate with the number of neurons.

- Fat chaotic attractors of $20-60 \%$ of the phase space dimension, implying large capacity for information processing and a huge repertoire of possible network states.

- High entropy production rates of 0.5-1 bit per spike per neuron. Compared to real sensory information provided to cortical neurons of about 1 bit per spike per neuron [93, 94], this implies that sensory information is overwritten at a similar rate as it is encoded.

- Activation of excitatory-inhibitory feedback loops intensifies the chaos, yet the dynamics in inhibitory and excitatory-inhibitory networks are qualitatively very similar.

- Temporal network chaos similar to spatiotemporal chaos. While a small fraction of neurons participated in the chaotic dynamics at one point in time, almost all neurons participated equally in the chaotic dynamics over time. 


\section{Networks of Rapid Theta Neurons}

\section{Variable Action Potential Onset Rapidness}

Cortical neurons were found to exhibit a much higher action potential (AP) onset rapidness than expected from standard biophysical neuron models [1]. This has raised fundamental physiological questions about the origin of this phenomenon (see Section 1.2). Another important issue regarding the understanding of information processing in the cortex is the impact of rapid AP initiation on the collective dynamics of cortical networks. This will be addressed in this chapter.

In the first section, we will introduce the rapid theta neuron model. Derived from the theta neuron model, the rapid theta neuron model combines the advantage of being exactly solvable with a variable AP onset rapidness. Thus, the approach developed in Chapter 2 can be directly applied to investigate the influence of AP onset rapidness on the collective network dynamics. The AP onset rapidness $r$ can be arbitrarily chosen in the rapid theta neuron model. As discussed in Section 1.2, reasonable values for standard neuron models like the Wang-Buzsáki model or the ConnorStevens model correspond to $r \approx 1 \ldots 3$. The theta neuron model is by construction equivalent to $r=1$. From experiments on cortical neurons, we expect a range between $r \approx 10 \ldots 100$ for cortical neurons. A comprehensive analysis of the dynamics of both inhibitory and excitatory networks of rapid theta neurons follows in Sections 3.3 and 3.4. We will demonstrate that the network dynamics strongly and qualitatively depends on the AP onset rapidness of the single neurons. For low $r$, the network dynamics are qualitatively similar to that of theta neurons studied in the previous chapter. An increase of $r$, however, can drive the network dynamics to the edge of chaos and in the large $r$ limit the networks exhibit stable chaos similar to that in leaky integrate and fire networks. Surprisingly, the firing statistics do not reflect this dramatic change in the network dynamics, including the pairwise spike correlations. In fact, the pairwise spike correlations are very weak independent of the AP onset rapidness of the neurons. Nevertheless, we will demonstrate that the statistics becomes entangled at the edge of chaos. This will be discussed in Sections 3.5 and 3.6 .

\subsection{Rapid Theta Neuron Model}

In order to examine the impact of the AP onset rapidness on the collective dynamics of cortical networks, a new neuron model with variable AP onset rapidness was developed, called the rapid theta neuron model (Fig. 3.1). The rapid theta neuron model combines the advantage of the theta neuron model of an analytic derivation of the phase-response curve with a freely variable AP onset rapidness $r$. For $r=1$, the rapid theta neuron model is equivalent to the theta neuron model and all results discussed in Chapter 2 hold. Increasing $r$ increases the time constant at the unstable fixed point $V_{U}$ leading to a larger instability and sharper AP initiation. The membrane time constant $\tau_{\mathrm{m}}$, the time constant at the stable fixed point $V_{S}$ remains unchanged. This is achieved by 'glueing' two 

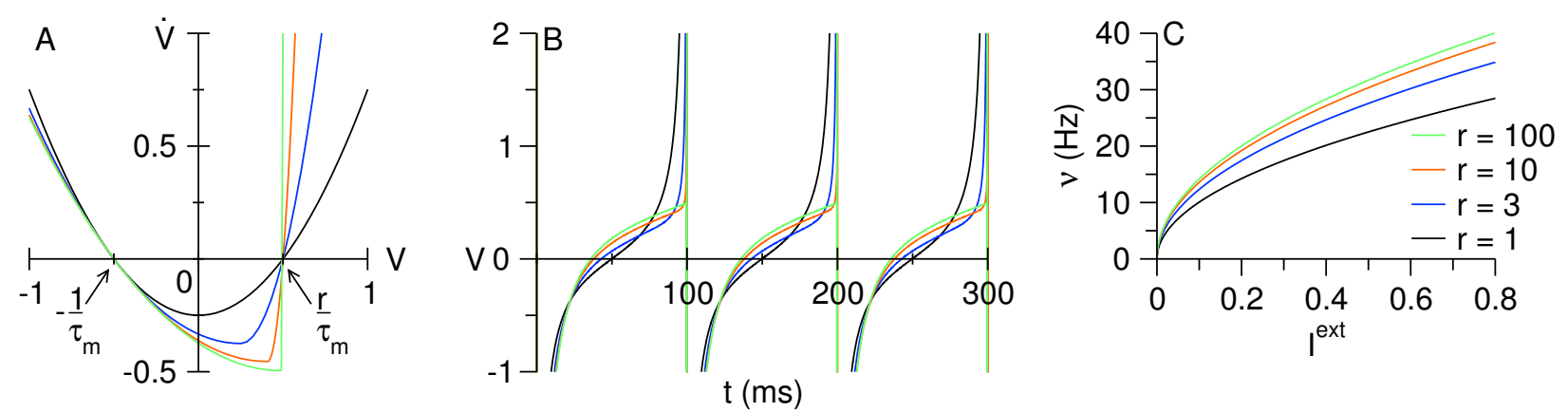

Figure 3.1 - Rapid theta neuron model. (A) Phase plot of the rapid theta neuron model, Eq. 3.1 and (B) voltage traces for three different AP onset rapidness $r$, (C) gain function $\left(F I\right.$-curve) $v=\frac{\sqrt{I^{\text {ext }}}}{\pi \tau_{\mathrm{m}}} \sqrt{\frac{2 r}{r+1}}$ for the suprathreshold input current $I^{\text {ext }}$.

parabolas smoothly together at $V_{G}$.In the dimensionless voltage representation, the resulting rapid theta neuron model is described by the differential equation

$$
\tau_{\mathrm{m}} \frac{\mathrm{d} V}{\mathrm{~d} t}= \begin{cases}a_{S}\left(V-V_{G}\right)^{2}-I_{T}+I(t) & V \leq V_{G} \\ a_{U}\left(V-V_{G}\right)^{2}-I_{T}+I(t) & V>V_{G}\end{cases}
$$

In this equation, $I_{T}$ denotes the rheobase current and $I(t)$ is the synaptic input current. The curvatures $a_{S, U}$ depend on the AP onset rapidness $r$ and together with $V_{G}$ and $I_{T}$ define the positions of the two branches of the parabolas. The glue point, denoted $V_{G}$, divides the phase space into a subthreshold $\left(V \leq V_{G}\right)$ and a suprathreshold $\left(V>V_{G}\right)$ part. At the stable fixed point $V_{S}$, the slope of the subthreshold parabola is set to $-1 / \tau_{\mathrm{m}}$ and at the unstable fixed point $V_{U}$ the slope of the suprathreshold is $r / \tau_{\mathrm{m}}$. This leads to the expressions

$$
\begin{aligned}
\frac{\partial \dot{V}\left(V_{S}\right)}{\partial V}=-1 & =2 a_{S}\left(V_{S}-V_{G}\right) \\
a_{S} & =\frac{1}{2} \frac{1}{\left(V_{G}-V_{S}\right)} \\
\frac{\partial \dot{V}\left(V_{U}\right)}{\partial V}=r & =2 a_{U}\left(V_{U}-V_{G}\right) \\
a_{U} & =\frac{r}{2} \frac{1}{\left(V_{U}-V_{G}\right)}
\end{aligned}
$$

The derivative of the voltage vanishes at the two fixed points for zero synaptic inputs $(I(t) \equiv 0)$. This defines the glueing point $V_{G}$ and the rheobase current $I_{T}$ :

$$
\begin{aligned}
\dot{V}\left(V_{S}\right)=0 & =a_{S}\left(V_{S}-V_{G}\right)^{2}-I_{T} \\
I_{T} & =\frac{V_{G}-V_{S}}{2} \\
\dot{V}\left(V_{U}\right)=0 & =a_{U}\left(V_{U}-V_{G}\right)^{2}-I_{T} \\
& =\frac{r V_{U}-V_{G}(r+1)+V_{S}}{2} \\
V_{G} & =\frac{r V_{U}+V_{S}}{r+1} .
\end{aligned}
$$


Without loss of generality, the stable and unstable fixed points are set to $V_{S}=-0.5$ and $V_{U}=$ +0.5 , yielding:

$$
\begin{aligned}
V_{G} & =\frac{1}{2} \frac{r-1}{r+1} \\
I_{T} & =\frac{1}{2} \frac{r}{r+1} \\
a_{S} & =\frac{r+1}{2 r} \\
a_{U} & =\frac{r(r+1)}{2}=r^{2} a_{S} .
\end{aligned}
$$

With Eq. (3.2)-3.5 the governing equation of the rapid theta neuron model (3.1) becomes

$$
\tau_{\mathrm{m}} \frac{\mathrm{d} V}{\mathrm{~d} t}= \begin{cases}\frac{r+1}{2 r}\left(V-\frac{1}{2} \frac{r-1}{r+1}\right)^{2}-I_{T}+I(t) & V \leq \frac{1}{2} \frac{r-1}{r+1} \\ \frac{r(r+1)}{2}\left(V-\frac{1}{2} \frac{r-1}{r+1}\right)^{2}-I_{T}+I(t) & V>\frac{1}{2} \frac{r-1}{r+1}\end{cases}
$$

A phase representation of the rapid theta neuron model similar to the classical theta neuron model is obtained with the transformation $\tan \frac{\theta}{2}=V-V_{G}$ and $\theta \in[-\pi, \pi]$, yielding

$$
\tau_{\mathrm{m}} \frac{\mathrm{d} \theta}{\mathrm{d} t}= \begin{cases}\frac{r+1}{2 r}(1-\cos \theta)+\left(I(t)-I_{T}\right)(1+\cos \theta) & \theta \leq 0 \\ \frac{r(r+1)}{2}(1-\cos \theta)+\left(I(t)-I_{T}\right)(1+\cos \theta) & \theta>0 .\end{cases}
$$

It can be seen that for $r=1$, the theta neuron model, Eq. (2.33), is recovered.

The exact solutions of the rapid theta neuron model for constant external currents and $\delta$ pulse coupling allow us to write down a phase representation with constant phase velocity similar to the one obtained for the theta neuron model. The solution of the governing differential equation in the dimensionless voltage representation (3.1) for constant synaptic currents $I(t) \equiv I_{T}+I$ is

$$
\begin{aligned}
\frac{1}{I} \frac{\mathrm{d} x}{1+\left(\frac{V-V_{G}}{\sqrt{I / a_{S, U}}}\right)^{2}} & =\frac{1}{\tau_{\mathrm{m}}} \mathrm{d} t \\
\frac{1}{I} \sqrt{I / a_{S, U}}\left[\arctan \left(\frac{V-V_{G}}{\sqrt{I / a_{S, U}}}\right)\right]_{V_{1}}^{V_{2}} & =\frac{t_{2}-t_{1}}{\tau_{\mathrm{m}}} \\
\arctan \left(\frac{V_{2}-V_{G}}{\sqrt{I / a_{S, U}}}\right) & =\arctan \left(\frac{V_{1}-V_{G}}{\sqrt{I / a_{S, U}}}\right)+\sqrt{I a_{S, U}} \frac{t_{2}-t_{1}}{\tau_{\mathrm{m}}} .
\end{aligned}
$$

This equation represents the solution for both branches of Eq. (3.1) separated by $V_{G}$ as before. For the subthreshold part $\left(V \leq V_{G}\right)$, the curvature is $a_{S}=\frac{r+1}{2 r}$ and for the suprathreshold part $\left(V>V_{G}\right)$, the curvature is $a_{U}=\frac{r(r+1)}{2}$. In the phase representation with phase $\phi \in[-\pi, \pi]$ and constant phase velocity, the phase evolution is given by

$$
\phi_{2}=\phi_{1}+\omega \frac{t_{2}-t_{1}}{\tau_{\mathrm{m}}}
$$


Identifying Eq. (3.8) and (3.9), enables us to derive the constant phase velocity $\omega$ and the 'glueing' point $\phi_{G}$ to define the transformation between the two representations

$$
\frac{\phi-\phi_{G}}{\omega}=\arctan \left(\frac{V-V_{G}}{\sqrt{I / a_{S, U}}}\right) \frac{1}{\sqrt{I a_{S, U}}} .
$$

During one complete cycle, the time $T_{S}$ spent in the subthreshold part $\left(V_{2}=V_{G}\right.$ and $\left.V_{1} \rightarrow-\infty\right)$ and the time $T_{U}$ spent in the suprathreshold part $\left(V_{2} \rightarrow \infty\right.$ and $\left.V_{1} \rightarrow V_{G}\right)$ can be taken from Eq. (3.8):

$$
T_{S}=\frac{\pi \tau_{\mathrm{m}}}{\sqrt{2 I(r+1) / r}} \quad \text { and } \quad T_{U}=\frac{\pi \tau_{\mathrm{m}}}{\sqrt{2 I(r+1) r}}
$$

The time spent in the subthreshold part is thus $T_{S} / T_{U}=r$ times as long as the one in the suprathreshold part. The total cycle length, or interspike interval, is thus

$$
\begin{aligned}
T^{\text {free }} & =(r+1) T_{U} \\
& =\frac{\pi \tau_{\mathrm{m}}}{\sqrt{I}} \sqrt{\frac{r+1}{2 r}} .
\end{aligned}
$$

The constant phase velocity is then

$$
\begin{aligned}
\omega & =\frac{2 \pi}{T^{\text {free }}} \\
& =\frac{2 \sqrt{I}}{\tau_{\mathrm{m}}} \sqrt{\frac{2 r}{r+1}}=\frac{2}{\tau_{\mathrm{m}}} \sqrt{I / a_{S}}
\end{aligned}
$$

The phase corresponding to the glueing point is

$$
\begin{aligned}
\phi_{G} & =-\pi+\omega T_{S} \\
& =\pi \frac{r-1}{r+1} .
\end{aligned}
$$

The constant phase velocity (3.12) and the glueing point (3.13) define the transformation 3.10) between the voltage representation and the phase representation:

$$
\begin{gathered}
\phi=\phi_{G}+ \begin{cases}\frac{2}{a_{S}} \arctan \left(\frac{V-V_{G}}{\sqrt{I / a_{S}}}\right) & V \leq V_{G} \\
\frac{2}{r a_{S}} \arctan \left(r \frac{V-V_{G}}{\sqrt{I / a_{S}}}\right) & V>V_{G}\end{cases} \\
V=V_{G}+ \begin{cases}\sqrt{I / a_{S}} \tan \left(a_{S} \frac{\phi-\phi_{G}}{2}\right) & \phi \leq \phi_{G} \\
\sqrt{I / r^{2} a_{S}} \tan \left(r a_{S} \frac{\phi-\phi_{G}}{2}\right) & \phi>\phi_{G} .\end{cases}
\end{gathered}
$$

This transformation between the two equivalent representations is now used to calculate the phase-transition curve $g(\phi)$, respectively the phase-response curve $Z(\phi)$. Receiving a $\delta$ pulse of strength $J$ leads to a step like change of the neuron's voltage $V^{+}=V^{-}+J$. If this change does not lead to a change from the subthreshold to the suprathreshold part or vice versa, the calculation of the phase-transition curve is straightforward. Some care needs to be taken, if the $\delta$ pulse does lead 
to such a change.

An inhibitory pulse $J<0$ can lead to a change from the suprathreshold to the subthreshold part. This happens if the neuron's phase is between $\phi_{G}$ and $\phi_{-}$. The phase-transition curve for inhibitory $\delta$ pulses of strength $J$ and constant external currents $I$ with the effective coupling $C=J / \sqrt{I}$ and $\phi_{-}=\phi_{G}+\frac{2}{r a_{S}} \arctan \left(r\left(V_{G}-J-V_{G}\right) / \sqrt{I / a_{S}}\right)=\phi_{G}-\frac{2}{r a_{S}} \arctan \left(r \sqrt{a_{S}} C\right)$ is

$$
\mathrm{g}_{-}(\phi)=\phi_{G}+ \begin{cases}\frac{2}{a_{S}} \arctan \left(\tan \left(a_{S} \frac{\phi-\phi_{G}}{2}\right)+\sqrt{a_{S}} C\right) & -\pi<\phi \leq \phi_{G} \\ \frac{2}{a_{S}} \arctan \left(\frac{1}{r} \tan \left(r a_{S} \frac{\phi-\phi_{G}}{2}\right)+\sqrt{a_{S}} C\right) & \phi_{G}<\phi<\phi_{-} \\ \frac{2}{r a_{S}} \arctan \left(\tan \left(r a_{S} \frac{\phi-\phi_{G}}{2}\right)+r \sqrt{a_{S}} C\right) & \phi_{-} \leq \phi<\pi .\end{cases}
$$

For excitatory $\delta$ pulses of strength $J>0$, the phase can change from the subthreshold to the suprathershold part if the phase is between $\phi_{+}$and $\phi_{G}$. The phase-transition curve for excitatory $\delta$ pulses of strength $J$ and constant external currents $I$ with the effective coupling $C=J / \sqrt{I}$ and $\phi_{+}=\phi_{G}-\frac{2}{a_{S}} \arctan \left(\sqrt{a_{S}} C\right)$ is

$$
g_{+}(\phi)=\phi_{G}+ \begin{cases}\frac{2}{a_{S}} \arctan \left(\tan \left(a_{S} \frac{\phi-\phi_{G}}{2}\right)+\sqrt{a_{S}} C\right) & -\pi<\phi \leq \phi_{+} \\ \frac{2}{r a_{S}} \arctan \left(r \tan \left(a_{S} \frac{\phi-\phi_{G}}{2}\right)+r \sqrt{a_{S}} C\right) & \phi_{+}<\phi<\phi_{G} \\ \frac{2}{r a_{S}} \arctan \left(\tan \left(r a_{S} \frac{\phi-\phi_{G}}{2}\right)+r \sqrt{a_{S}} C\right) & \phi_{G} \leq \phi<\pi\end{cases}
$$

The phase-response curve is $Z_{ \pm}(\phi)=g_{ \pm}(\phi)-\phi$. Thus, the infinitesimal phase-response curve is the same for both excitatory and inhibitory pulses, since $\phi_{ \pm} \rightarrow \phi_{G}$ for $C \rightarrow 0$ :

$$
Z(\phi) \stackrel{C \rightarrow 0}{\simeq} C \begin{cases}\frac{2 \sqrt{a_{S}}}{a_{S}} \frac{1}{1+\tan \left(a_{S} \frac{\phi-\phi_{G}}{2}\right)^{2}}=\frac{1+\cos \left(a_{S}\left(\phi-\phi_{G}\right)\right)}{\sqrt{a_{S}}} & -\pi<\phi \leq \phi_{G} \\ \frac{2 r \sqrt{a_{S}}}{r a_{S}} \frac{1}{1+\tan \left(r a_{S} \frac{\phi-\phi_{G}}{2}\right)^{2}}=\frac{1+\cos \left(r a_{S}\left(\phi-\phi_{G}\right)\right)}{\sqrt{a_{S}}} & \phi_{G} \leq \phi<\pi .\end{cases}
$$

To investigate the collective dynamics of networks of rapid theta neurons, the derivative $d(\phi)$ of the phase-transition curve is needed for the single spike Jacobians, Eq. (2.15). The derivative in case of inhibitory pulses is

$$
d_{-}(\phi)= \begin{cases}\frac{\tan \left(a_{S} \frac{\phi-\phi_{G}}{2}\right)^{2}+1}{\left(\tan \left(a_{S} \frac{\phi-\phi_{G}}{2}\right)+\sqrt{a_{S}} C\right)^{2}+1} & -\pi<\phi \leq \phi_{G} \\ \frac{\tan \left(r a_{S} \frac{\phi-\phi_{G}}{2}\right)^{2}+1}{\left(\frac{1}{r} \tan \left(r a_{S} \frac{\phi-\phi_{G}}{2}\right)+\sqrt{a_{S}} C\right)^{2}+1} & \phi_{G}<\phi<\phi_{-} \\ \frac{\tan \left(r a_{S} \frac{\phi-\phi_{G}}{2}\right)^{2}+1}{\left(\tan \left(r a_{S} \frac{\phi-\phi_{G}}{2}\right)+r \sqrt{a_{S}} C\right)^{2}+1} & \phi_{-} \leq \phi<\pi .\end{cases}
$$



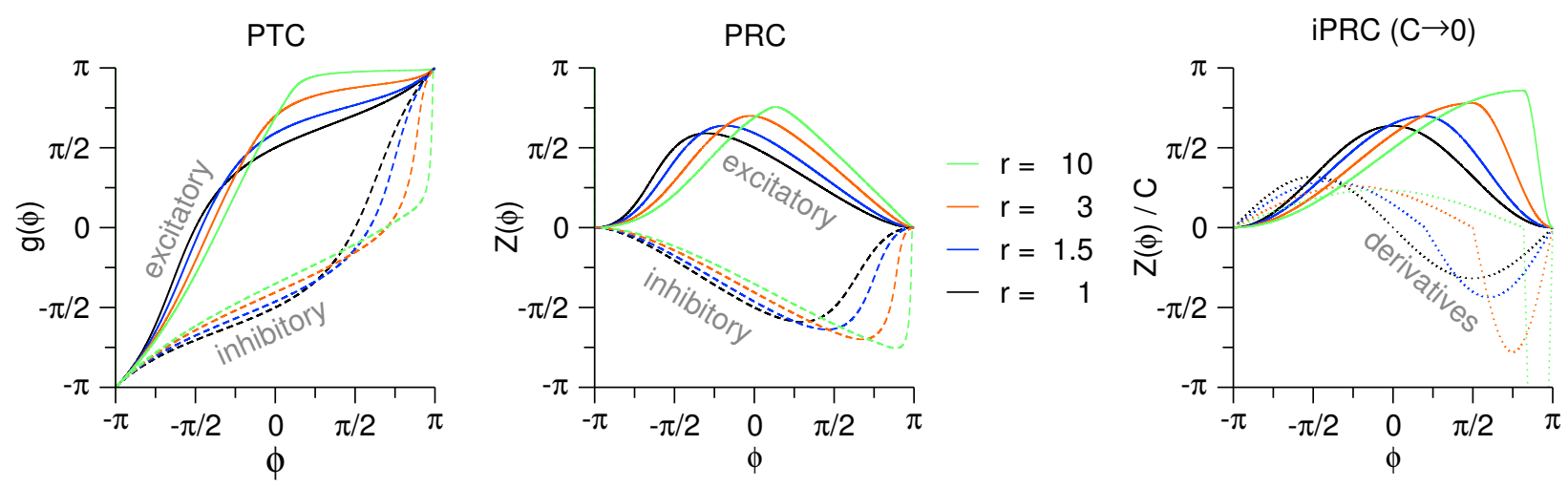

Figure 3.2 - Phase-transition curve (PTC), phase-response (PRC) and infinitesimal phase-response (iPRC) of the rapid-theta neuron model with AP onset rapidness $r$. The PTC (left panel) and PRC=PTC$\phi$ (middle panel) are shown for inhibitory coupling $C=-1$ (dashed lines, Eq. (3.16) and excitatory coupling $C=+1$ (solid lines, Eq. 3.17)). In the right panel are displayed the iPRC (Eq. (3.18) ) and its derivative (Eq. (3.21).

The derivative of the phase-transition curve in the case of excitatory pulses is

$$
d_{+}(\phi)= \begin{cases}\frac{\tan \left(a_{S} \frac{\phi-\phi_{G}}{2}\right)^{2}+1}{\left(\tan \left(a_{S} \frac{\phi-\phi_{G}}{2}\right)+\sqrt{a_{S}} C\right)^{2}+1}-1 & -\pi<\phi \leq \phi_{+} \\ \frac{\tan \left(a_{S} \frac{\phi-\phi_{G}}{2}\right)^{2}+1}{\left(r \tan \left(a_{S} \frac{\phi-\phi_{G}}{2}\right)+r \sqrt{a_{S}} C\right)^{2}+1}-1 & \phi_{+}<\phi<\phi_{G} \\ \frac{\tan \left(r a_{S} \frac{\phi-\phi_{G}}{2}\right)^{2}+1}{\left(\tan \left(r a_{S} \frac{\phi-\phi_{G}}{2}\right)+r \sqrt{a_{S}} C\right)^{2}+1}-1 & \phi_{G} \leq \phi<\pi .\end{cases}
$$

The derivative of the phase-response curve is $Z_{ \pm}^{\prime}(\phi)=d_{ \pm}(\phi)-1$ and the derivative of the infinitesimal phase-response curve is

$$
Z^{\prime}(\phi) \stackrel{C \rightarrow 0}{\simeq}-C \begin{cases}\sqrt{a_{S}} \sin \left(a_{S}\left(\phi-\phi_{G}\right)\right) & -\pi<\phi \leq \phi_{G} \\ r \sqrt{a_{S}}-\sin \left(r a_{S}\left(\phi-\phi_{G}\right)\right) & \phi_{G} \leq \phi<\pi .\end{cases}
$$

The phase-transition curves (PTC, $g(\phi)$, Eq. (3.16) and (3.17)), the phase response curves (PRC, $Z(\phi)=g(\phi)-\phi)$ and the infinitesimal phase-response curves (iPRC, Eq. (3.18) ) of the rapid theta neuron model are displayed in Fig. 3.2. The iPRC of the theta neuron $(r=1)$ is fully symmetric, whereas for increasing AP onset rapidness $r$ the iPRC becomes more and more asymmetric. In the limit $r \rightarrow \infty$ it becomes monotonously increasing/decreasing and one might expect that this can qualitatively change the collective network dynamics.

\subsection{Balanced State in Rapid Theta Neuron Networks}

The derived exact expressions of the phase-transition curves, Eq. (3.16), (3.17), and their derivatives, Eq. (3.19), 3.20) allow for the direct application of the approach introduced in Chapter 2 for precise simulations and the quantification of the collective dynamics of rapid theta neurons. 

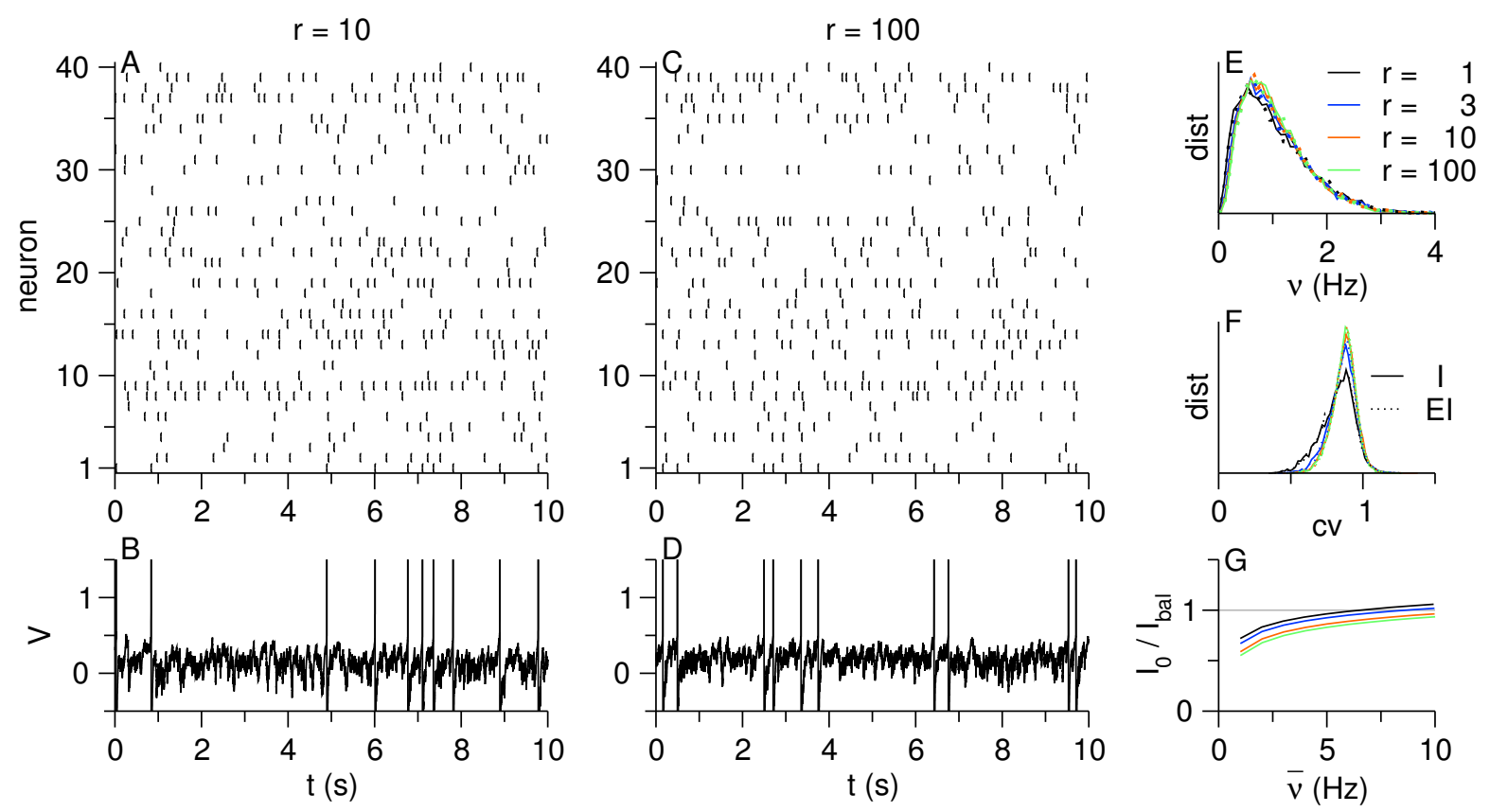

Figure 3.3 - Characteristics of the balanced state in rapid theta neuron networks. (A,C) spike trains of 40 randomly chosen neurons, (B,D) voltage trace of first randomly chosen neuron, (E) firing rate distributions for different AP onset rapidness $r$ and their excitatory-inhibitory counterparts (dotted lines), (F) coefficients of variation distributions as in E, $(\mathrm{G})$ suprathreshold external current $I_{0}$ in relation to estimated balanced current $I_{\mathrm{bal}}=\bar{v} J_{0} \tau_{\mathrm{m}}$ from balance equation 2.19) versus target average firing rate $\bar{v}\left(I_{0} / I_{\mathrm{bal}}=1\right.$ would be perfect fit), (parameters: $N=10000, K=100, \bar{v}=10 \mathrm{~Hz}, \tau_{\mathrm{m}}=10 \mathrm{~ms}$; all simulation with identical network realizations).

We will study the exact same networks as in Chapter 2 but now composed of rapid theta neurons with variable AP onset rapidness. An AP onset rapidness $r=1$ is equivalent to the theta neuron model, thus the results of Chapter 2 are recovered. In these theta neuron networks, we have observed two phase transitions from the asynchronous balanced state to a synchronous state. One transition to a synchronous irregular state occurred at a critical connectivity $K_{c}$ and one to synchronous regular state at a critical excitatory-inhibitory feedback loop strength $\varepsilon_{c}$. In rapid theta neuron networks with larger AP onset rapidness, both phase transitions were shifted to much higher critical parameters and disappeared entirely for large $r \gtrsim 10$ (Fig. 3.4). This indicates that a higher AP onset rapidness, increases the parameter range in which inhibitory and excitatory-inhibitory networks exhibit the balanced state.

The firing statistics of the studied networks exhibited the characteristic features of the balanced state and were basically insensitive to the AP onset rapidness $r$ of the single neurons (Fig. $\mathbf{3 . 3}$ ). The seemingly random firing patterns of 40 neurons in Fig. 3.3A,C display the typical asynchronous irregular state independent of $r$. Two individual voltage traces represent the characteristic strong voltage fluctuations of the neurons (Fig. 3.3B,D). Furthermore, the firing rate distributions and coefficient of variation distributions were broad indicating strong heterogeneity in the networks independent of $r$ (Fig. 3.3E,F). Due to the specific parametrization of the coupling strengths in excitatory-inhibitory networks, Eq. (2.31), they were also identical in both types of networks. This allows for a quantitative comparison of the two network types. 

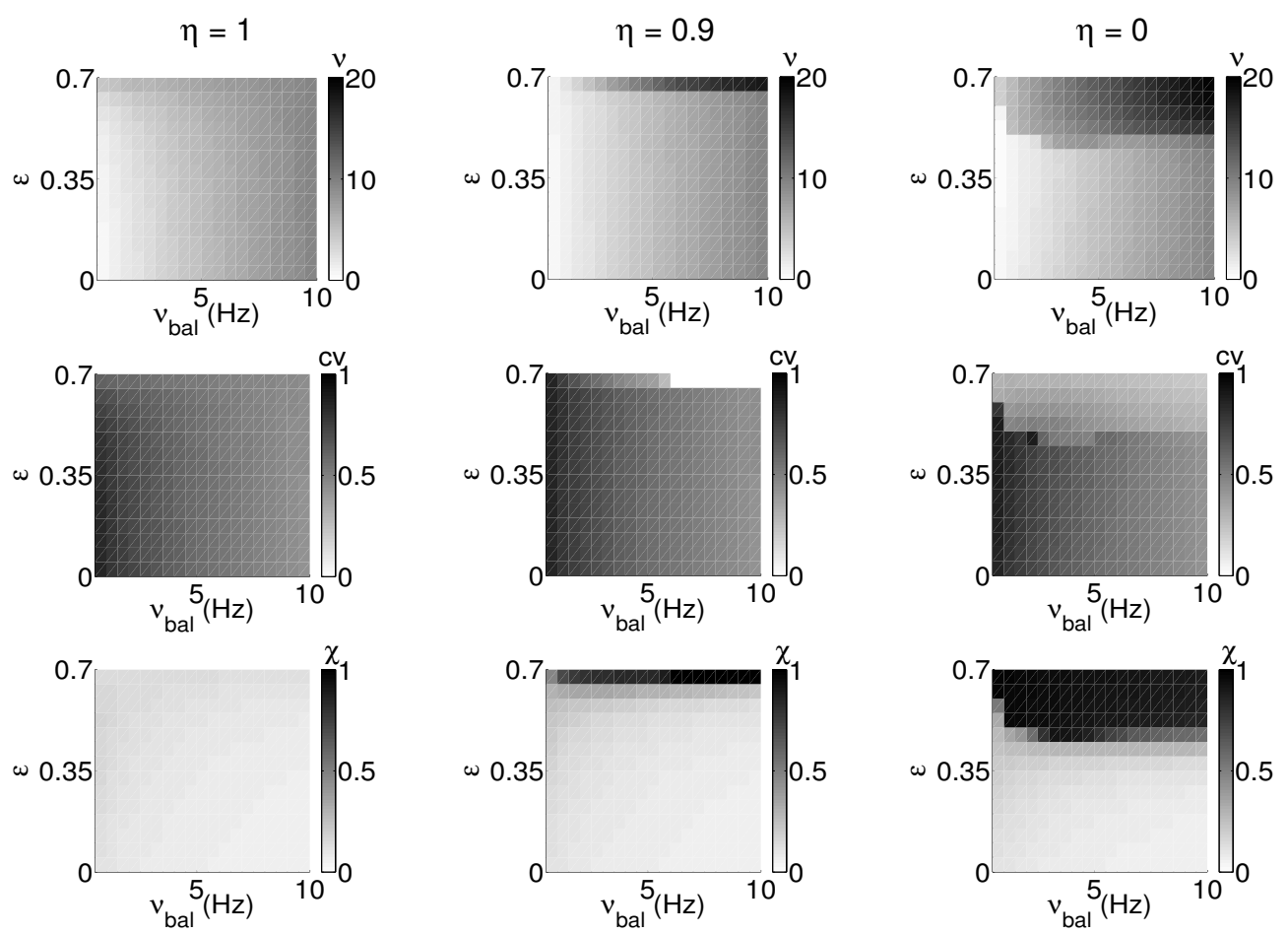

(a) $r=3$
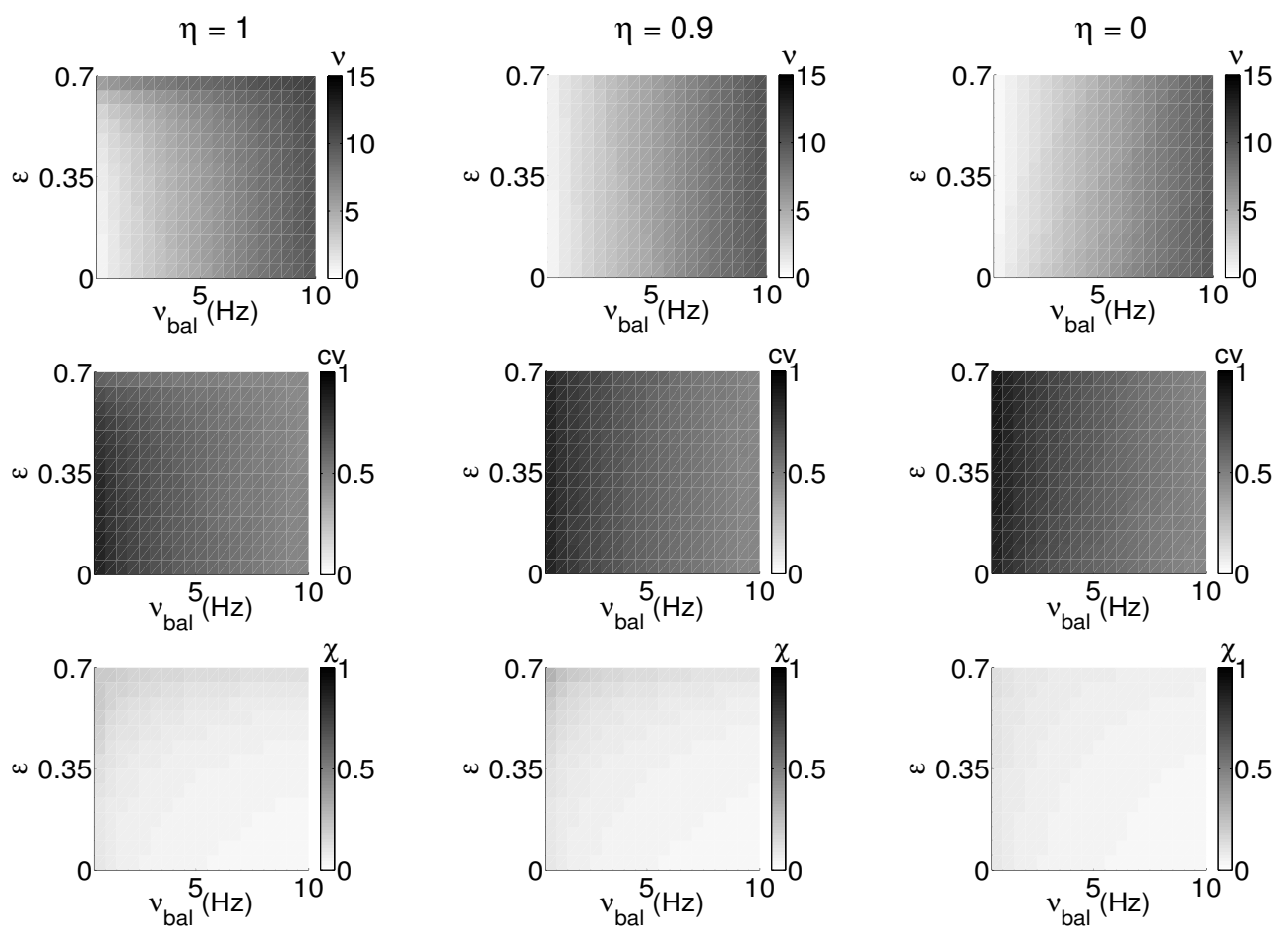

(b) $r=10$

Figure 3.4 - Phase diagrams of excitatory-inhibitory networks for different ratios $\eta=J_{E E} / J_{I E}$. (see coupling matrix (2.31) and Fig. 2.9 for $r=1$ ) Columns from left to right: $\eta=1,0.9,0$, from top to bottom: average firing rate $\bar{v}$, coefficient of variation $\mathrm{cv}$ and synchrony measure $\chi$; on the $\mathrm{x}$-axis is plotted the target average firing rate according to the balance equation 2.25) and on the y-axis the excitatory-inhibitory feedback loop activation $\varepsilon$, (parameters: $N_{E}=8000, N_{I}=2000, K=100, J_{0}=1, \tau_{\mathrm{m}}=10 \mathrm{~ms}$; displayed are averages of 10 runs with different network realizations). 

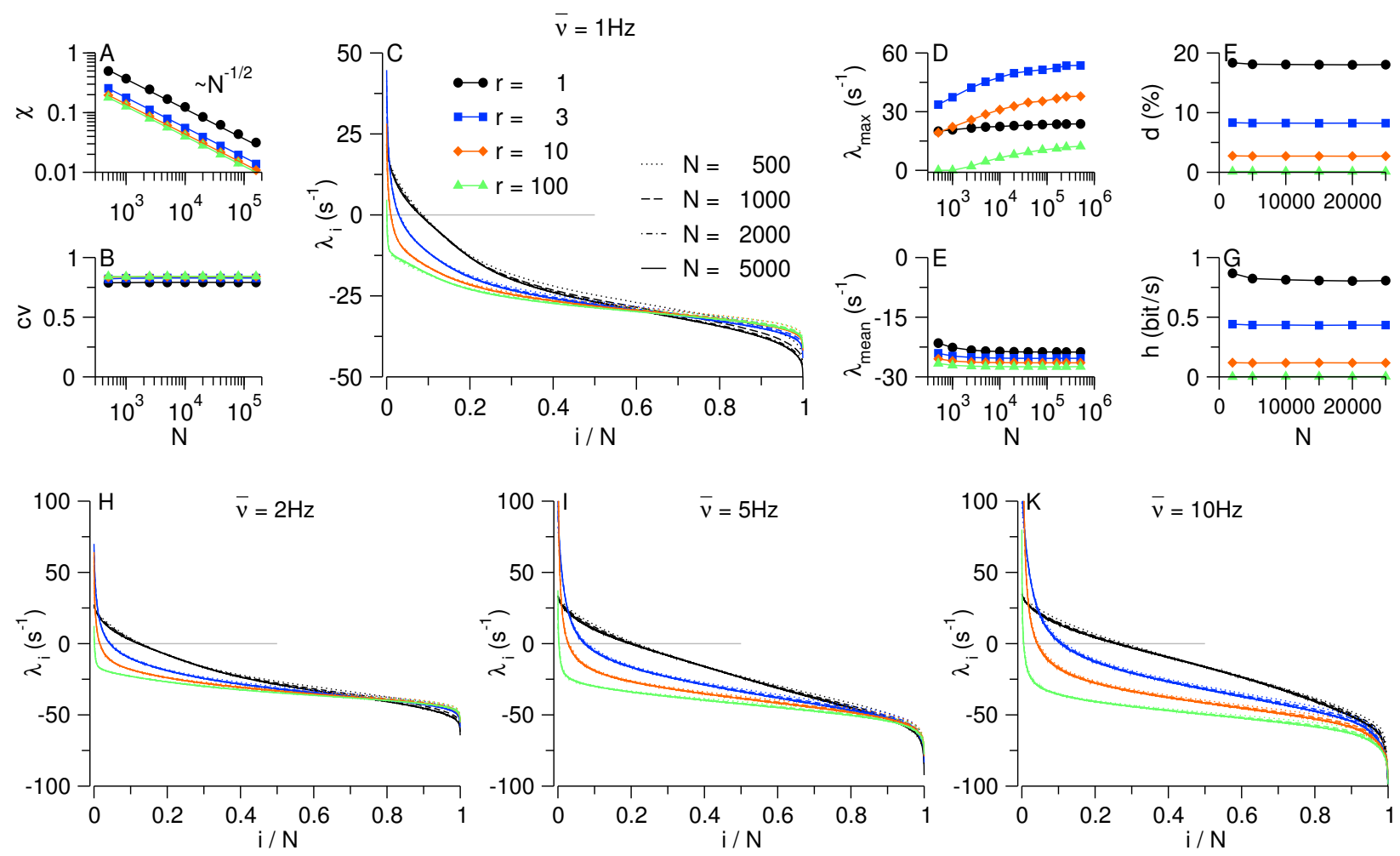

Figure 3.5 - Extensive dynamics in balanced inhibitory networks of $N$ rapid theta neurons with variable AP onset rapidness $r$. (A) Synchrony measure $\chi$ (straight line: $\chi \sim 1 / \sqrt{N}$ ), (B) average coefficient of variation cv, (C) full Lyapunov spectra $\left\{\lambda_{i}\right\}$ for different network sizes $N$, (D) largest Lyapunov exponent $\lambda_{\max }=\lambda_{1}$, (E) mean Lyapunov exponent $\lambda_{\text {mean }}=\frac{1}{N} \sum_{i} \lambda_{i}$, (F) attractor dimension in percent of phase space dimension $d=D / N,(\mathrm{G})$ average entropy production rate per neuron $h=H / N,(\mathrm{H})$-(K) full Lyapunov spectra for different firing rates $\bar{v},\left(K=100, J_{0}=1, \tau_{\mathrm{m}}=10 \mathrm{~ms}\right.$; averages of 10 runs with different network realizations).

\subsection{Dynamics of Inhibitory Networks}

An interesting feature of randomly connected theta neuron networks in the balanced state is the extensivity of the dynamics (Fig. (2.10)). Figure 3.5 shows that this extensivity is insensitive to the AP onset rapidness of the single neurons. The decreasing synchrony measure $\chi \sim N^{-1 / 2}$ with network size $N$ and the constantly high coefficient of variation $c v \approx 0.8$ indicate the typical asynchronous irregular balanced state in the networks which was independent of the AP onset rapidness $r$ of the single neurons (Fig. 3.5A,B). The dynamics of the networks, however, showed a strong dependence on $r$ indicated by completely different Lyapunov spectra (Fig. 3.5C). Nevertheless, for all $r$ the Lyapunov spectra were invariant to the number of neuron in the networks $N$. They all converged to a unique shape when plotting the Lyapunov exponents $\left\{\lambda_{i}\right\}$ versus the rescaled index $i / N$ (Fig. 3.5 C,H-K). Therefore, the number of positive Lyapunov exponents, the entropy production rate $H$, Eq. (1.5), and the attractor dimension $D$, Eq. (1.6), increased linearly with number of neurons. They are thus extensive quantities and it is well justified to define the relative attractor dimension $d=D / N$ and the average entropy production rate per neuron $h=H / N$ (Fig. 3.5F,G).

A difference in the collective dynamics of neurons with higher AP onset rapidness compared to theta neurons $(r=1)$ emerged for the largest Lyapunov exponent (Fig. 3.5D). The largest Lyapunov exponent $\lambda_{\max }$ increased with an increasing number of neurons $N$ and converged only slowly for 

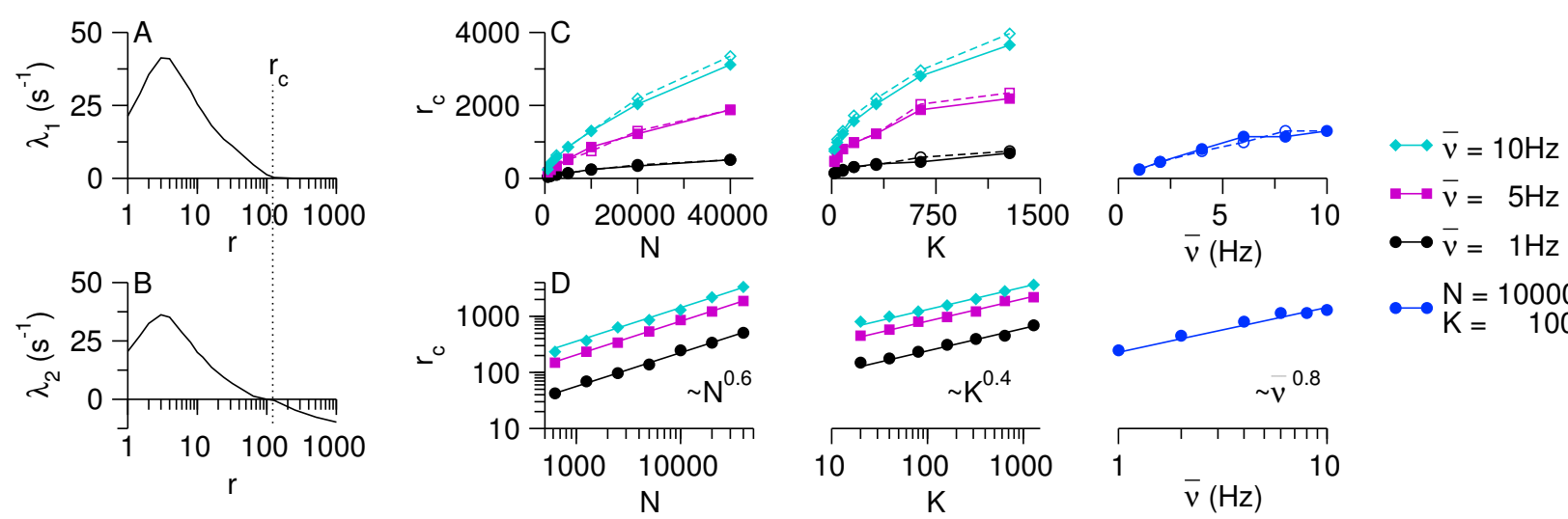

Figure 3.6 - Critical AP onset rapidness $r_{\mathrm{c}}$ between stable and chaotic dynamics in balanced inhibitory networks. (A) Largest Lyapunov exponent $\lambda_{\max }=\lambda_{1}$ vanishes at $r_{\mathrm{c}}(\operatorname{lin}-\log$ plot, $N=2000, K=100)$, (B) second Lyapunov exponents $\lambda_{2}$ crosses zero at $r_{\mathrm{c}}$ (lin-log plot, $N=2000, K=100$ ), (C) the critical AP rapidness $r_{\mathrm{c}}$ separating chaotic dynamics (below) from stable dynamics (above) versus number of neurons $N$ for $K=100$, versus number of synapses per neuron $K$ for $N=10000$ and versus average firing rate $\bar{v}$ for $N=10000$ and $K=100$, dashed lines: numerical calculations with double precision, solid lines: numerical calculations with quadruple precision, (D) filled circles: quadruple precision data from (C) in log-log plots, solid lines: fitted curves with given power law, (other parameters: $J_{0}=1, \tau_{\mathrm{m}}=10 \mathrm{~ms}$; averages of 10 runs with different network realizations).

extremely large networks.

Conversely, the increase of $\lambda_{\max }$ with $N$ raises the question whether it can also vanish for small $N$. This would correspond to a phase transition from stable to chaotic dynamics. This occurred indeed for very large AP onset rapidness $r$. In Fig. 3.5D, for example, it can be seen that for $r=100$, the largest Lyapunov exponent vanishes for networks with less than about 2000 neurons. In Figure 3.6A,B are displayed the first and the second Lyapunov exponent in such a network for increasing $\mathrm{AP}$ onset rapidness. At the critical AP onset rapidness of $r_{c} \approx 100$ in this example, the first Lyapunov exponent vanishes and the second Lyapunov exponent changes from positive to negative. This indicates a phase transition from chaotic to stable dynamics. Such networks are at the edge of chaos.

A large critical AP onset rapidness $r_{c}$, setting the networks at the edge of chaos, was found for all parameters in balanced inhibitory networks (Fig 3.6C). It appears to exhibit the scaling behavior $r_{c} \sim N^{0.6} K^{0.4} \bar{v}^{0.8}$ (Fig 3.6D). Because the critical AP onset rapidness can become very large, the results of the numerical calculations with double precision (dashed lines) were verified with calculations using quadruple precision (solid lines). If there does exist stable dynamics for (possibly very large) AP onset rapidness, this implies that the information in the spike pattern could actually be maintained because there is no loss of information. Theta neuron networks $(r=1)$, on the other hand, exhibit a strikingly high loss of information, as was demonstrated in Chapter2. So, how does the rate of information loss in the chaotic dynamics depend on the AP onset rapidness of the neurons in detail?

Increasing the AP onset rapidness of the neurons reduced the intensity of the chaos in balanced networks (Fig. 3.7). The synchrony measure $\chi$ slightly decreased and the coefficient of variation cv slightly increased for moderate increases of the AP onset rapidness (Fig. 3.7A,B). In the same range up to $r \approx 10$ the largest Lyapunov exponent $\lambda_{\max }$ increased (Fig. 3.7 C). Further increasing $r$, however, led to a reduction of $\lambda_{\max }$ while $\chi$ and $\mathrm{cv}$ were constant. The observed peak in the 

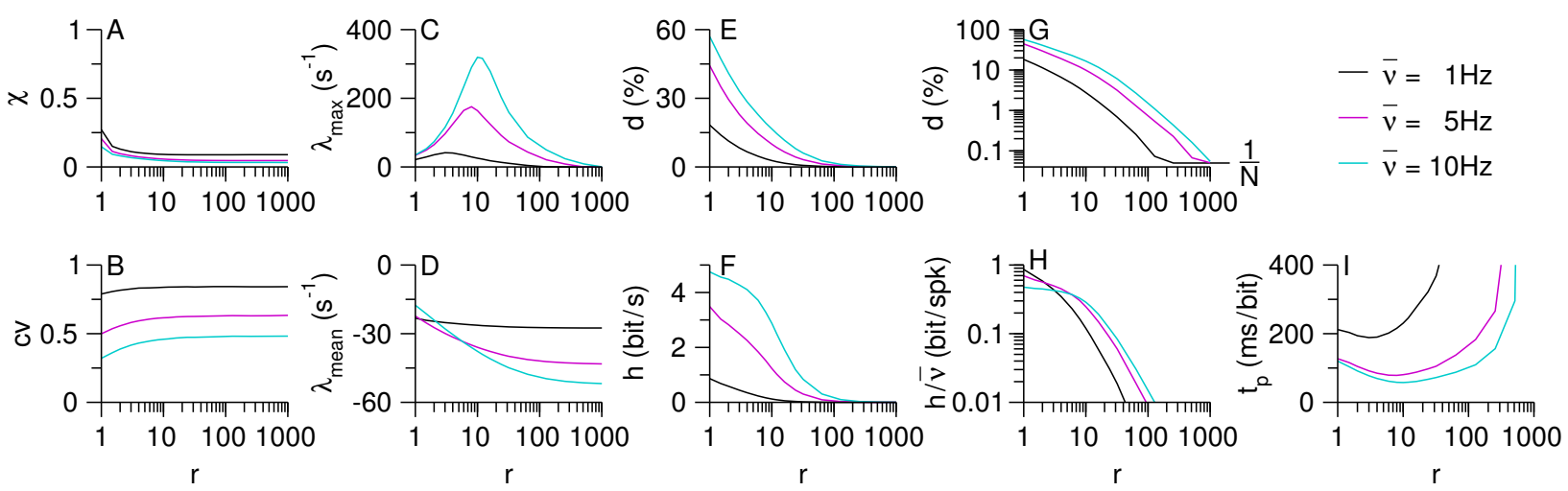

Figure 3.7 - AP onset rapidness $r$ strongly influences the dynamics of balanced inhibitory networks (AP rapidness $r$ on logarithmic scale): (A) Synchrony measure $\chi,(B)$ average coefficient of variation cv, (C) largest Lyapunov exponent $\lambda_{\max }=\lambda_{1}$, (D) mean Lyapunov exponent $\lambda_{\text {mean }}=\frac{1}{N} \sum_{i} \lambda_{i}$, (E) attractor dimension in percent of phase space dimension $d=D / N,(\mathrm{~F})$ average entropy production rate per neuron $h=H / N$, $(\mathrm{G})$ attractor dimension in log-log plot, $(\mathrm{H})$ average entropy production rate per neuron per spike in log-log plot, (I) maximal predictive time $t_{p} \propto D / H$, (parameters: $N=2000, K=100, \bar{v}=1 \mathrm{~Hz}, J_{0}=1, \tau_{\mathrm{m}}=10 \mathrm{~ms}$; averages of 10 runs with different network realizations).

largest Lyapunov exponent depending on $r$ will find an explanation in the investigation of the dynamics on the connectivity $K$. As opposed to the largest Lyapunov exponent exhibiting a peak, the attractor dimension and the entropy production rate decreased monotonously with increasing $r$ (Fig. 3.7E-H). A decreasing attractor dimension indicates a reduced information capacity of the networks. Assuming that the input information provided to the network does not exceed the maximal capacity, the reduced entropy production rate then means that the information is depleted more slowly in networks with larger AP onset rapidness.

The transition from the asynchronous irregular to a synchronous irregular state while increasing the connectivity $K$ disappeared for AP onset rapidness $r>1$. We increased the average number of synapses per neuron $K$ while keeping the probability $p=K / N$ of a connection between two neurons fixed. Theta neuron networks $(r=1)$ exhibit a transition from the asynchronous to a synchronous state as discussed in Chapter 2. For $r>1$, this transition did not occur or was at least shifted to much higher values of $K$. In the asynchronous state, the firing statistics were largely insensitive to an increase of $K($ Fig. $3.8 \mathbf{A}, \mathbf{B})$ as expected from the mean-field theory of the balanced state. The attractor dimension and entropy production rate seemed to converge for large $K$ (Fig. 3.8F,G). An interesting behavior showed the largest Lyapunov exponent. It first increased linearly with $K$ and then at some $K$, depending on $r$, it converged to a constant. This change in the dynamics was not reflected in the network statistics, as the synchrony measure and coefficient of variation do not vary at these values of $K$. Also, for higher $r$ the range expanded in which the largest Lyapunov exponent increased with $K$. This change of the network dynamics will be studied further.

The transition to the synchronous state was shifted to much higher $K$ for larger $r$ (Fig. 3.9 (a)). Extending the observed range of $K$ up to 10000 synapses per neuron reveals the onset of this transition for $r=3$ at around $K_{c} \approx 2000$, which is a ten times higher critical connectivity. For $r \geq 10$, the transition to synchrony could not be observed in the considered parameter range. These observations imply that the transition to synchrony at a critical connectivity $K_{c}$ was shifted to extremely high values for large $r$ and might even disappear.

The analysis of networks with fixed number of neurons and increasing number of synapses 

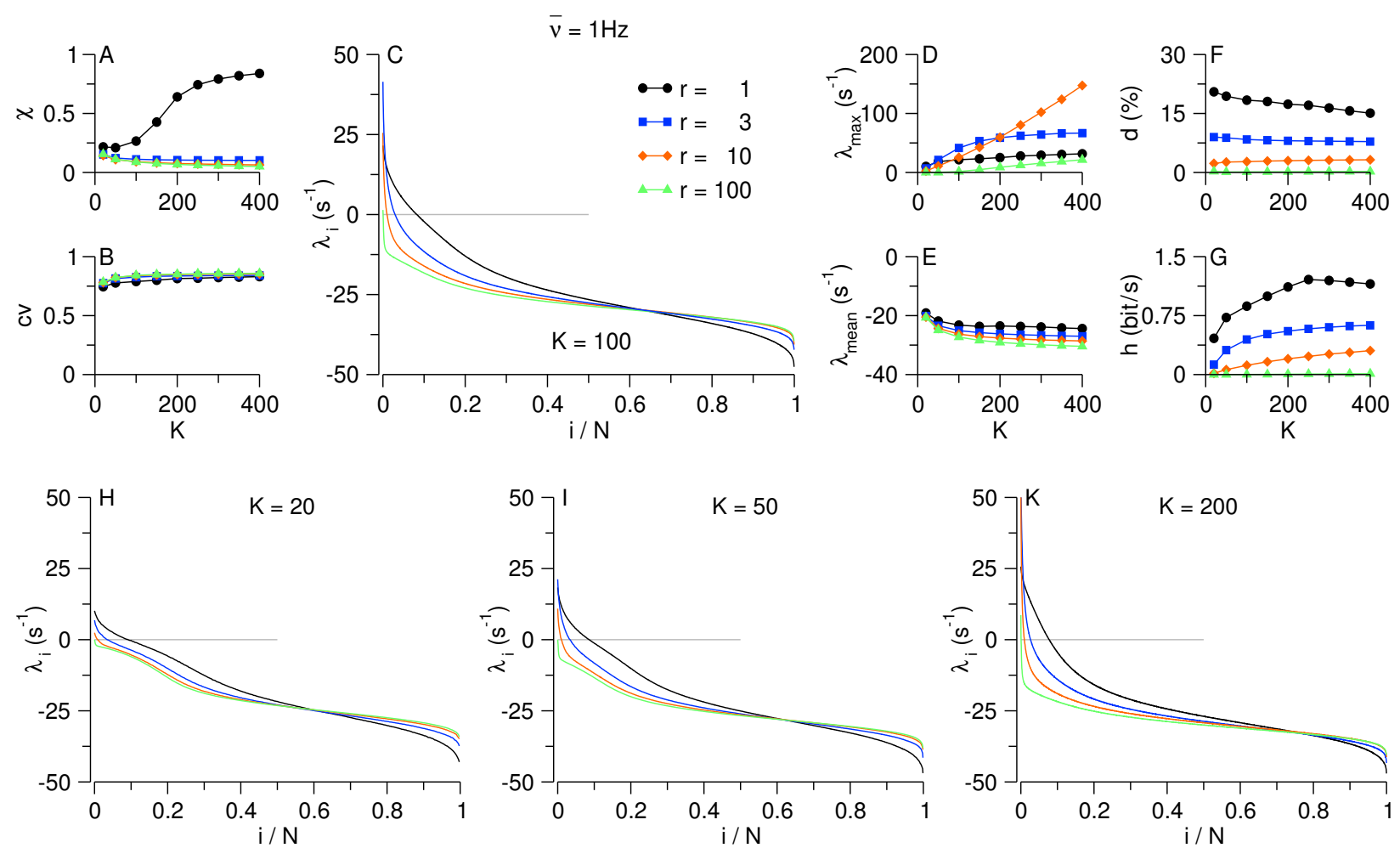

Figure 3.8 - Dynamics of dense networks for different AP rapidness $r$ and varied number of synapses $K$. (A) Synchrony measure $\chi$, (B) average coefficient of variation cv, (C) full Lyapunov spectra $\left\{\lambda_{i}\right\}$ for different network sizes $N$, (D) largest Lyapunov exponent $\lambda_{\max }=\lambda_{1}$, (E) mean Lyapunov exponent $\lambda_{\text {mean }}=\frac{1}{N} \sum_{i} \lambda_{i},(\mathrm{~F})$ attractor dimension in percent of phase space dimension $d=D / N,(\mathrm{G})$ average entropy production rate per neuron $h=H / N,(\mathrm{H})-(\mathrm{K})$ full Lyapunov spectra for different number of synapses $K$, (parameters: $N=20 K, \bar{v}=1 \mathrm{~Hz}, J_{0}=1, \tau_{\mathrm{m}}=10 \mathrm{~ms}$; averages of 10 runs with different network realizations).

further proves that the transition to synchrony disappears for large $r$ (Fig. 3.9(b)). The number of synapses per neuron $K$ was varied up to the all-to-all coupled case for $K=N=10000$. Networks of theta neurons $(r=1)$ showed a very similar dependence on $K$ compared to Fig. 3.9 (a). It indicates that at around $K_{c} \approx 200$, theta neuron networks begin to act similar to the all-to-allcoupled networks. They are in a synchronous irregular state. For rapid theta neuron networks there are two differences: (i) the critical connectivity increases strongly with increasing AP onset rapidness, and (ii) all-to-all coupled network are in a partially synchronous or asynchronous regular state.

One can suspect from Fig. 3.9 (b) that for $r=100$, the fully coupled networks are in a stable splay state. This demonstrates that the AP onset rapidness qualitatively changes the dynamics of inhibitory balanced networks.

This qualitatively different dynamics depending on the AP onset rapidness explains the observed peak in the largest Lyapunov exponent versus AP onset rapidness. We have found two different scaling regimes of the largest Lyapunov exponent $\lambda_{\max }$ with respect to the number of synapses $K$ per neuron in Fig. 3.9. For clarity, this is shown again in Fig. 3.10A. Increasing $K$ first leads to a linear increase of $\lambda_{\max }(K)$ up to a typical $K_{r}$ that depends on $r$. A further increase of $K$ leads to a plateau in $\lambda_{\max }(K)$. Interestingly, the largest Lyapunov exponent scales like $\lambda_{\max }\left(K<K_{r}\right) \sim 1 / r$ in the first (increasing) regime and scales like $\lambda_{\max }\left(K>K_{r}\right) \sim r$ in the second (plateau) regime. 

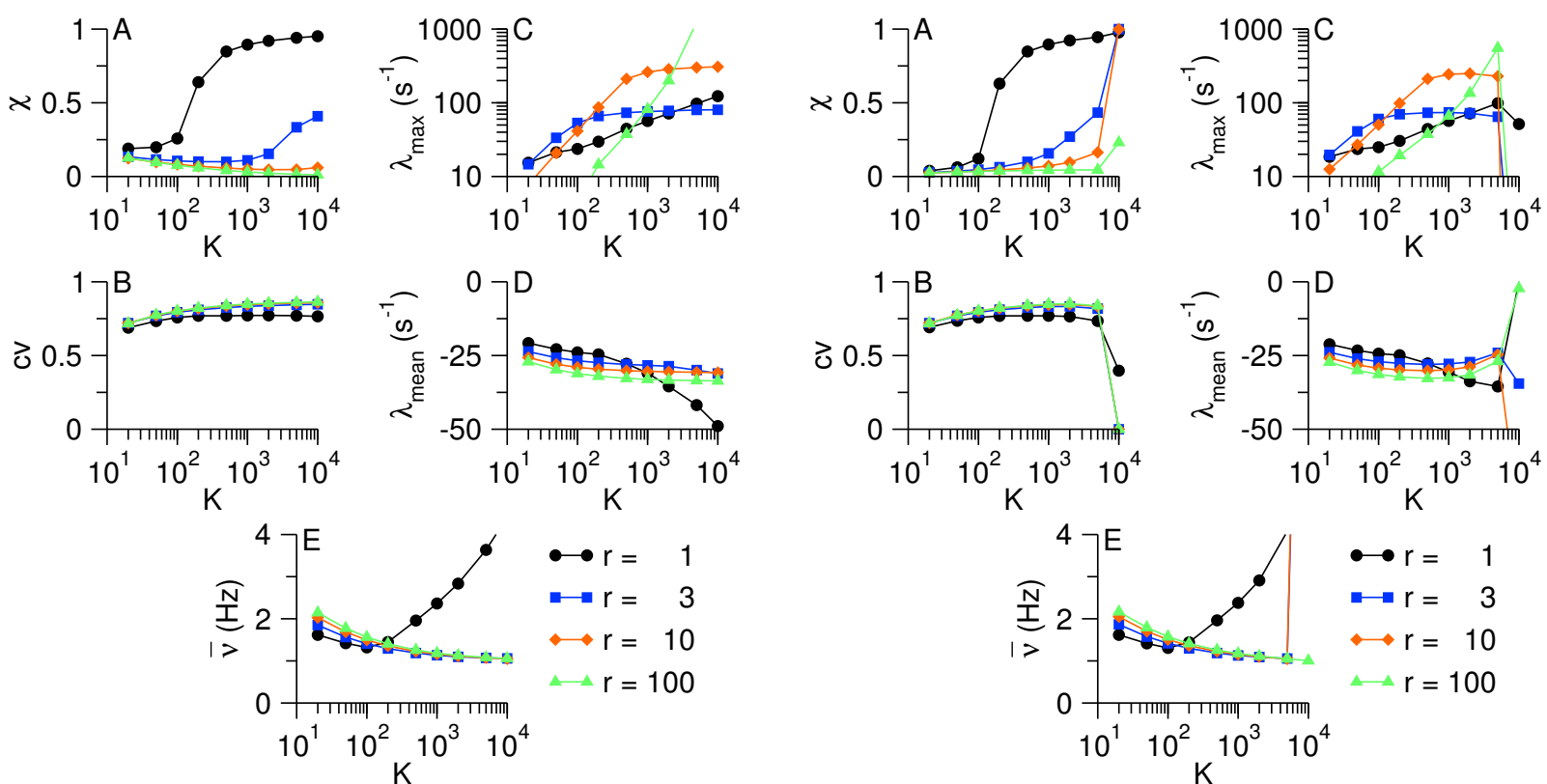

(a) Dense networks with $p=K / N=0.05$ fixed.

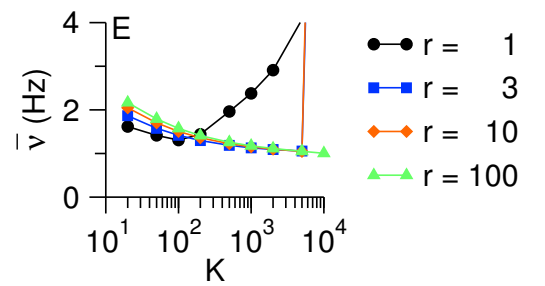

(b) Dense networks with $N=10^{4}$ fixed.

Figure 3.9 - Transition from asynchronous irregular to synchronous irregular state shifted with increasing AP onset rapidness $r$ : (A) Synchrony measure $\chi$, (B) average coefficient of variation $\mathrm{cv},(\mathrm{C})$ largest Lyapunov exponent $\lambda_{\max }=\lambda_{1}$, (D) mean Lyapunov exponent $\lambda_{\text {mean }}=\frac{1}{N} \sum_{i} \lambda_{i}$, (E) actual networkaveraged firing rate $\bar{v}$ (parameters: $K=100, J_{0}=1, \tau_{\mathrm{m}}=10 \mathrm{~ms}$; the input currents were here chosen to fulfill the balance equation (2.19) for the target average firing rate $\bar{v}_{\mathrm{bal}}=1 \mathrm{~Hz}$; averages of 10 runs with different network realizations).

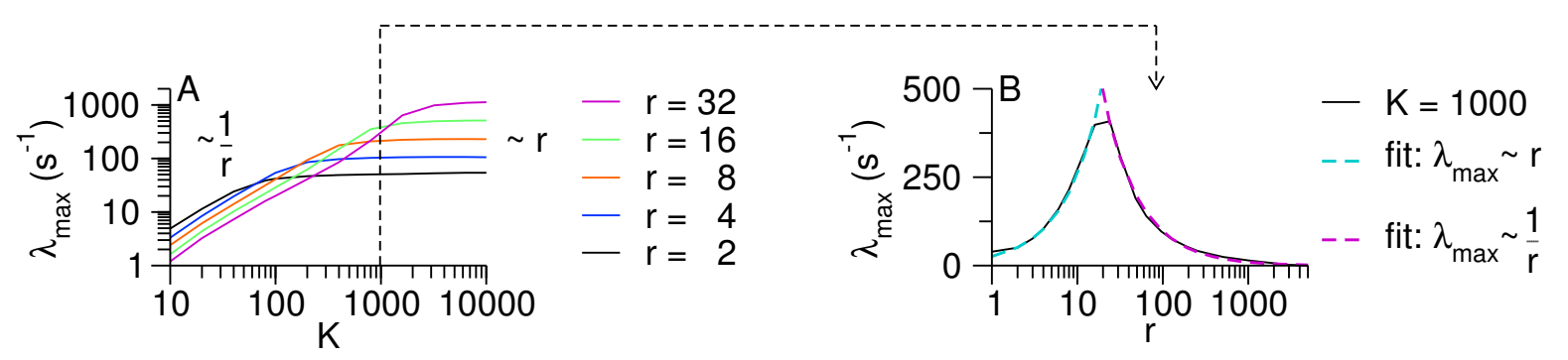

Figure 3.10 - Different scaling regimes of largest Lyapunov exponent with number of synapses explain the peak in largest Lyapunov exponent versus AP onset rapidness. (A) largest Lyapunov exponent $\lambda_{\max }$ versus number of synapses $K$ for fixed AP onset rapidness $r$, (B) $\lambda_{\max }$ versus $r$ for fixed $K=1000$ and fits to the two different scaling regimes, (parameters: $N=131072, \bar{v}=1 \mathrm{~Hz}, J_{0}=1, \tau_{\mathrm{m}}=10 \mathrm{~ms}$; averages of 10 runs with different network realizations). 
Now $K_{r}$ also increases with $r$. Therefore, keeping $K$ fixed as in Fig. 3.7, leads to a transition from the second (plateau) to the first (increase) regime and $\lambda_{\max }$ first increases with $r$, then saturates and finally decreases as $1 / r$. The numerical fits displayed in Fig. 3.10 agree very well with the two different regimes. Thus, the peak in $\lambda_{\max }(r)$ results from a qualitative change of the dynamics for increasing AP onset rapidness.

Even though the largest Lyapunov exponent changes its scaling behavior which indicates a qualitative change in the network dynamics, one should note that the firing statistics as well as the entropy production rate and attractor dimension did not change their qualitative characteristics (Fig. 3.7). The entropy production rate and attractor dimension decreased monotonously with increasing AP onset rapidness and were hardly affected by an increase of the number of synapses or number of neurons in the networks.

\subsubsection{The Limit of Instantaneous AP Initiation}

In the limit of infinitely fast AP onset rapidness $r \rightarrow \infty$, the AP initiation becomes instantaneous and the rapid theta neuron model in this limit is similar to the leaky integrate and fire (LIF) model. The difference is that, as opposed to the LIF model, there is no finite reset and threshold value in the rapid theta neuron. More importantly, the LIF model neglects the dynamic AP generation since a spike is emitted (virtually) when the voltage crosses the threshold potential. This is similar to the case of $r \rightarrow \infty$ in the rapid theta neuron model.

The limit of instantaneous AP initiation $(r \rightarrow \infty)$ can be approached in different ways. On the one hand, we showed for finite inhibitory networks of size $N$ and connectivity $K$ that the dynamics becomes stable upon increasing the AP onset rapidness beyond a finite but large value $r_{c}$ (Fig. 3.6). Thus, in the limit of $r \rightarrow \infty$, the dynamics would be stable even when taking the limit of large $N$ and $K$ afterwards. On the other hand, we showed that taking the limit of large $N$ and $K$ first, yields a scaling of the largest Lyapunov exponent $\lambda_{\max } \sim r$ (Fig. 3.9). Thus, taking then the limit $r \rightarrow \infty$ appears to yield a largest Lyapunov exponent that approaches infinity.

We conclude that the dynamics in the large network and connectivity limit strongly depends on the order in which this limit and the limit of instantaneous AP initiation are taken. Depending on the order of these limits, the collective network dynamics would either be characterized as stable $\left(\lambda_{\max }=0\right)$ or as extremely chaotic $\left(\lambda_{\max }=\infty\right)$.

\subsection{Dynamics of Excitatory-Inhibitory Networks}

Increasing the excitatory coupling in balanced networks of rapid theta neurons intensified the chaos (Fig. 3.11). The balanced state was again characterized by low synchrony measure $\chi$ and a high coefficient of variation cv. Upon activating the excitatory-inhibitory feedback loops with $\varepsilon$, the largest Lyapunov exponent $\lambda_{\max }$ and the attractor dimension $d$ increased and exceeded the values from inhibitory networks for large $\varepsilon$ (dotted lines). The entropy production rate $h$ increased linearly with $\varepsilon$ and exceeded that of inhibitory networks for large $\varepsilon$ as well. The difference in the dynamics of excitatory-inhibitory rapid theta neurons $(r>1)$ compared to theta neurons $(r=1)$ is that the largest Lyapunov exponent increased strongly with $\varepsilon$, and the attractor dimension and entropy production could exceed the values of inhibitory networks. In theta neurons, the largest Lyapunov exponent was hardly affected by increasing $\varepsilon$ and the attractor dimension and entropy production remained below the values of inhibitory networks. 

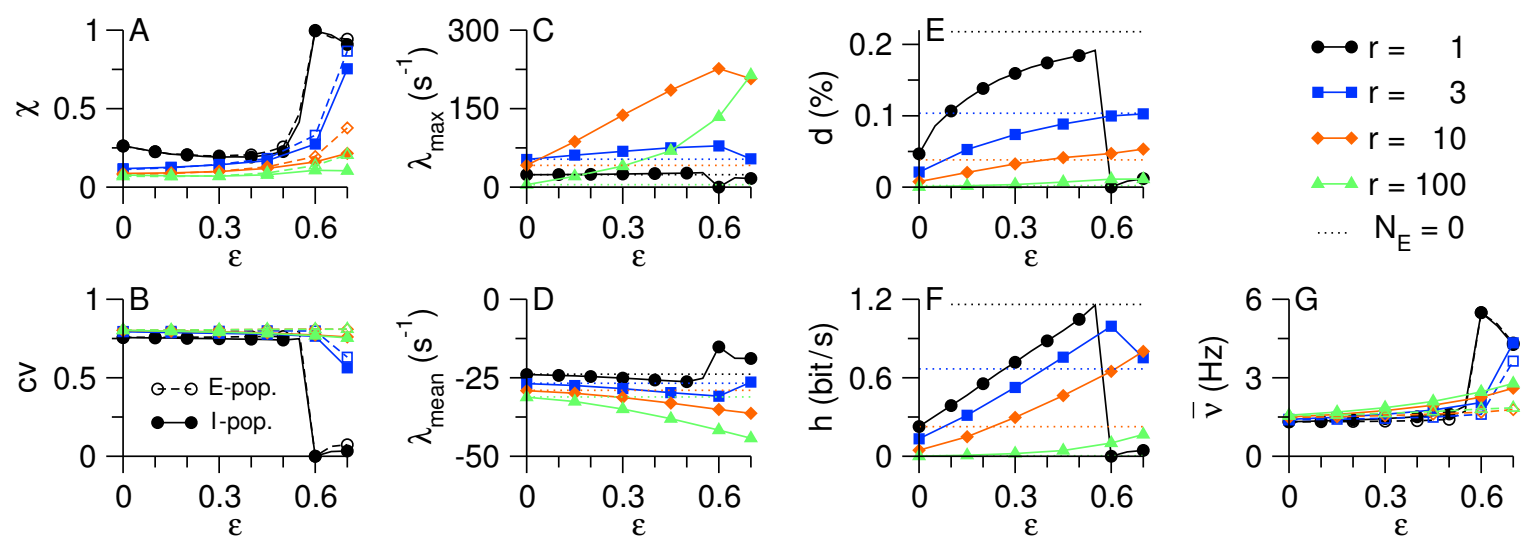

Figure 3.11 - Dynamics of excitatory-inhibitory networks with different AP onset rapidness $r$, while activating the excitatory-inhibitory feedback loops with $\varepsilon$. (values of inhibitory networks as dotted lines for comparison): (A) Synchrony measure $\chi$, (B) average coefficient of variation cv, (C) largest Lyapunov exponent $\lambda_{\max }=\lambda_{1}$, (D) mean Lyapunov exponent $\lambda_{\text {mean }}=\frac{1}{N} \sum_{i} \lambda_{i}$, (F) attractor dimension in percent of phase space dimension $d=D / N,(\mathrm{G})$ average entropy production rate per neuron $h=H / N,(\mathrm{C}-\mathrm{F})$ dashed lines: values from isolated inhibitory networks $\left(N_{E}=0\right),(\mathrm{G})$ actual network-averaged firing rate $\bar{v}$, (parameters: $N_{E}=8000, N_{I}=2000, K=100, J_{0}=1, \tau_{\mathrm{m}}=10 \mathrm{~ms}, \eta=0.9$; the input currents were here chosen to fulfill the balance equation 2.25) for the target average firing rate $\bar{v}_{\mathrm{bal}}=1 \mathrm{~Hz}$; averages of 10 runs with different network realizations).

The transition from the asynchronous irregular state to a synchronized regular state disappeared for high AP onset rapidness $r$. In theta neuron networks $(r=1)$, we observed this transition in which the dynamics changed from chaotic to stable at a critical excitatory-inhibitory feedback loop strengths $\varepsilon_{c}$. The transition is characterized by an abruptly increasing synchrony measure $\chi$, an increasing mean firing rate $\bar{v}$, and a decreasing coefficient of variation $\mathrm{cv}$. This appeared in theta neuron networks $(r=1)$ at a critical excitatory-inhibitory feedback loop strength $\varepsilon_{c} \approx 0.5$. When increasing the AP onset rapidness, this transition is shifted to larger $\varepsilon_{c}$. For $r=3$ it is approximately $\varepsilon_{c} \approx 0.6$ and for $r>10$ the transition completely disappears and the balanced state is stable in the entire parameter range.

In inhibitory networks, we observed a transition from chaotic to stable dynamics with increasing AP onset rapidness. Such networks near the edge of chaos are always driven into the chaotic regime when increasing the excitation. This can be seen for $r=100$ in Fig. 3.11. Equivalent inhibitory networks and excitatory-inhibitory networks with passive excitatory neurons $(\varepsilon=0)$ exhibit a zero largest Lyapunov exponent and are at the edge of chaos for $r=100$. Upon activating the excitatory-inhibitory feedback loops with $\varepsilon>0$, a chaotic dynamics sets in, characterized by positive Lyapunov exponents, attractor dimension and entropy production rate. Thus, the excitatory-inhibitory feedback loops can set a dynamic edge of chaos in rapid theta neuron networks. This might serve as a dynamic mechanism for switching between the two computationally highly interesting regimes-stable and chaotic dynamics.

The dynamics of chaotic excitatory-inhibitory networks was qualitatively very similar to the dynamics of inhibitory networks (Fig. 3.12). For various excitatory-inhibitory feedback loop strengths $\varepsilon$ and average firing rates $\bar{v}$, the largest Lyapunov exponent exhibited a pronounced peak and the attractor dimension and entropy production rate decreased monotonously for increasing $r$. This was also observed in inhibitory networks as discussed in the previous section. The influence of the AP onset rapidness on the dynamics of both inhibitory and excitatory-inhibitory 

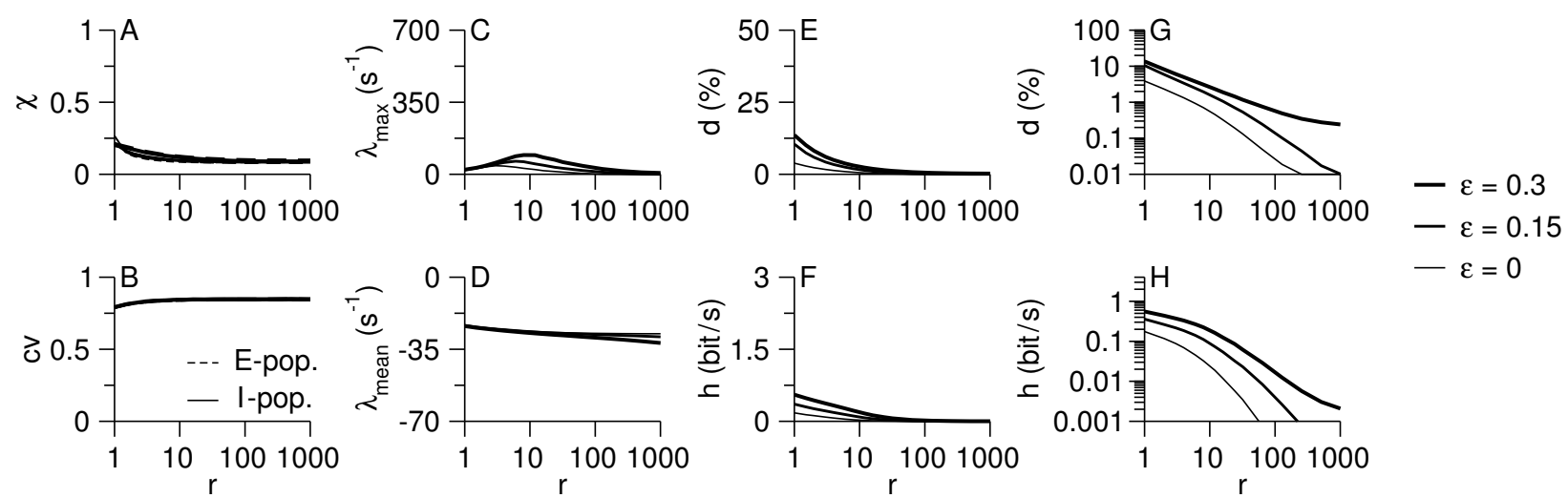

$-\varepsilon=0$

(a) $\bar{v}_{E}=\bar{v}_{I}=1 \mathrm{~Hz}$
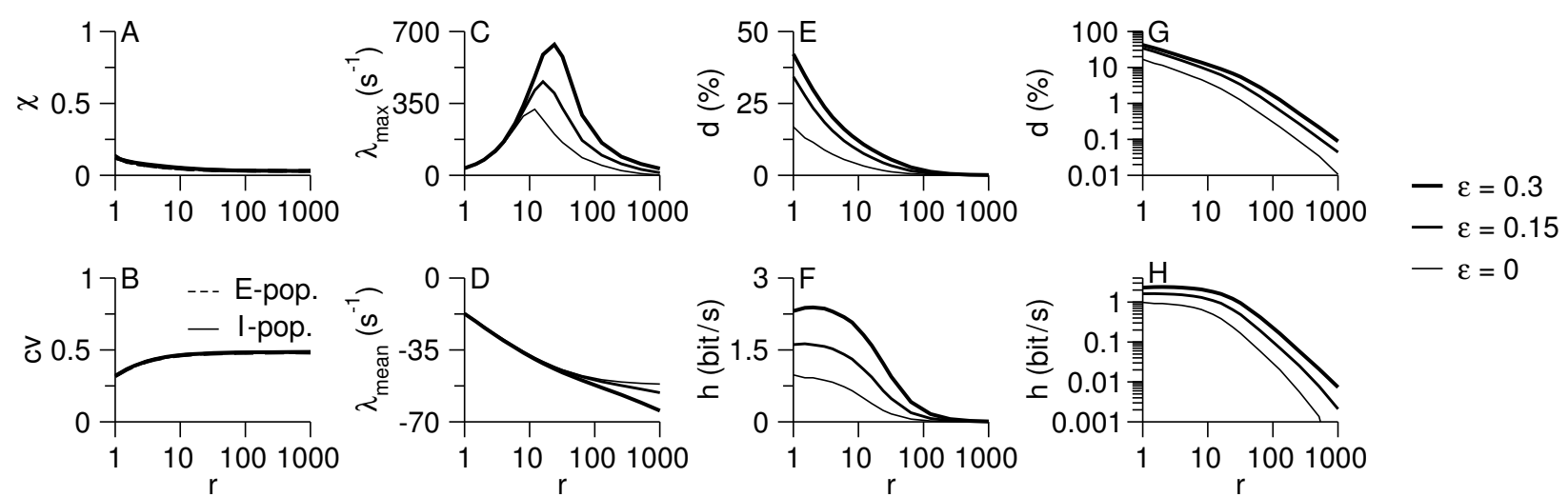

(b) $\bar{v}_{E}=\bar{v}_{I}=10 \mathrm{~Hz}$

Figure 3.12 - AP onset rapidness $r$ strongly influences the dynamics of excitatory-inhibitory balanced networks with excitatory-inhibitory feedback strength $\varepsilon$. (AP rapidness on logarithmic scale): (A) Synchrony measure $\chi,(\mathrm{B})$ average coefficient of variation $\mathrm{cv}$, (C) largest Lyapunov exponent $\lambda_{\max }=\lambda_{1}$, (D) mean Lyapunov exponent $\lambda_{\text {mean }}=\frac{1}{N} \sum_{i} \lambda_{i}$, (E) attractor dimension in percent of phase space dimension $d=D / N,(\mathrm{~F})$ average entropy production rate per neuron $h=H / N,(\mathrm{G})$ attractor dimension in $\log -\log$ plot, $(\mathrm{H})$ average entropy production rate per neuron per spike in log-log plot, (parameters: $N_{E}=8000$, $N_{I}=2000, K=100, J_{0}=1, \eta=0.9, \tau_{\mathrm{m}}=10 \mathrm{~ms}$; averages of 10 runs with different network realizations).

networks is summarized in Fig. 3.13. Networks of exclusively inhibitory neurons and networks of $20 \%$ inhibitory and $80 \%$ excitatory neurons share the same largest Lyapunov exponent when the excitatory neurons are passive $(\varepsilon=0)$. The average entropy production rate per neuron per spike is reduced accordingly to one fifth in excitatory-inhibitory networks. Upon activating the excitatoryinhibitory feedback loops $(\varepsilon>0)$, the chaos becomes more intense resulting in a higher Lyapunov exponent and entropy production rate. Qualitatively, however, the dynamics of excitatoryinhibitory networks is very similar to inhibitory networks. The only difference in the excitatoryinhibitory networks is that for increased excitation the networks seem to stay in the chaotic regime even for very larger AP onset rapidness (Fig. 3.12 and 3.13). We were therefore wondering how the critical AP onset rapidness $r_{c}$ changes with increasing excitation in the networks.

The strength of the excitatory-inhibitory feedback loops exponentially increased the critical AP onset rapidness defining the edge of chaos (Fig. 3.14). For networks with an average firing rate of $\bar{v}=1 \mathrm{~Hz}$, one can suspect from Fig. 3.12 (a) that the transition to stable dynamics might disappear for strong excitatory-inhibitory feedback loops. This is indeed the case, indicated by a diverging 

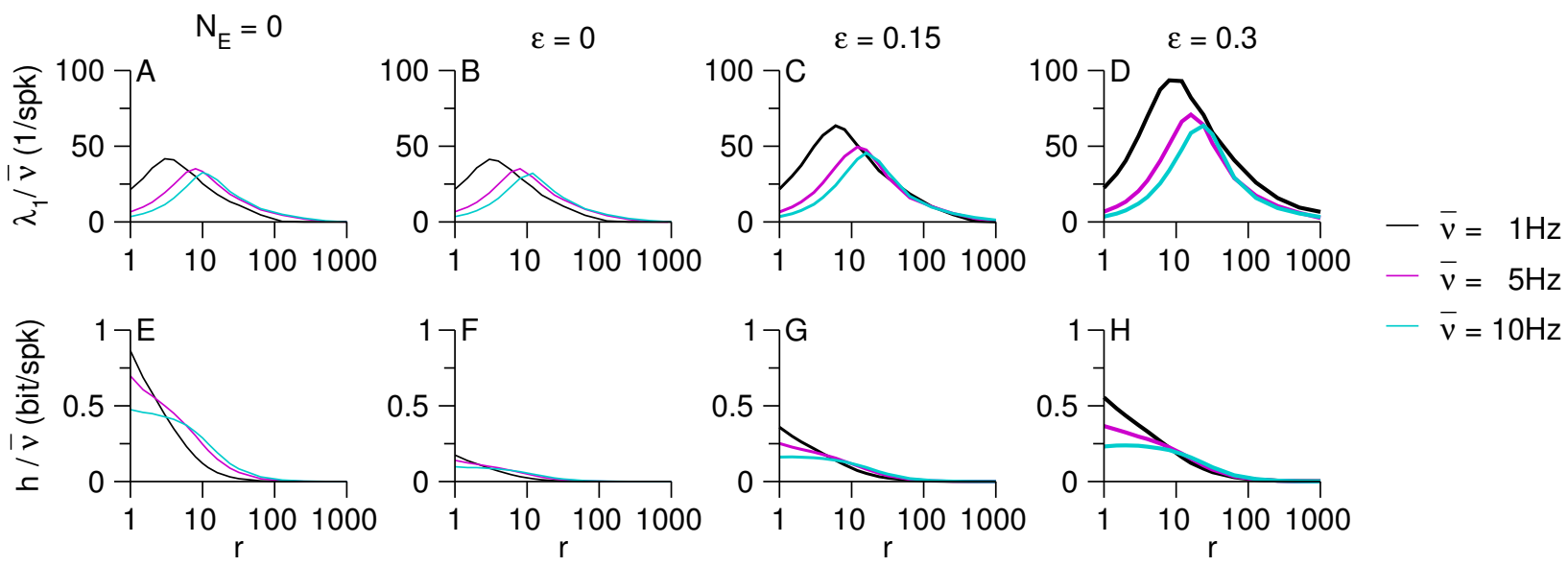

Figure 3.13 - Comparison of AP onset rapidness-influence on the dynamics of balanced neuron networks. (A),(E) Inhibitory networks, (B),(F), excitatory-inhibitory networks with passive excitatory neurons $\varepsilon=0,(\mathrm{C}),(\mathrm{G})$ excitatory-inhibitory feedback strength $\varepsilon=0.15$, (D), (H) $\varepsilon=0.3$, (A)-(D) largest Lyapunov exponent $\lambda_{\max }=\lambda_{1}$ divided by average firing rate $\bar{v},(\mathrm{E})-(\mathrm{H})$ average entropy production rate per neuron $h=H / N$, (parameters: $N_{E}=8000, N_{I}=2000, K=100, \bar{v}_{E}=\bar{v}_{I}, J_{0}=1, \eta=0.9, \tau_{\mathrm{m}}=10 \mathrm{~ms}$; averages of 10 runs with different network realizations).
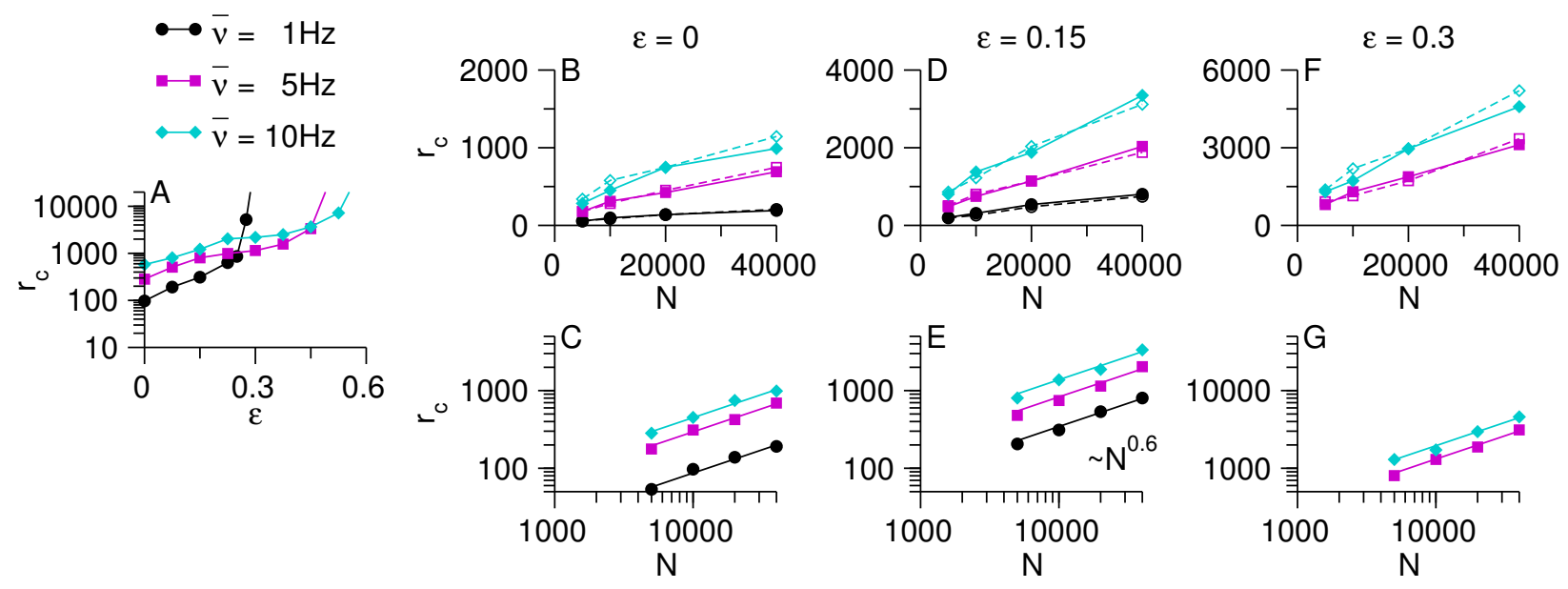

Figure 3.14 - Critical AP onset rapidness $r_{\mathrm{c}}$ at which the networks are at the edge of chaos. (A) Critical AP rapidness $r_{\mathrm{c}}$ versus excitatory-inhibitory feedback loop strength $\varepsilon$ (the simulations were stopped if the lagest Lyapunov exponent was still positive at $r=10^{5}$ ), (B,C) Critical AP rapidness $r_{\mathrm{c}}$ versus number of neurons $N$ when excitatory neurons are passive $(\varepsilon=0),(\mathrm{D}, \mathrm{E})$ for $\varepsilon=0.15$ and $(\mathrm{F}, \mathrm{G})$ for $\varepsilon=0.3$, dashed lines: numerical calculation with double precision, solid lines: numerical calculation with quadruple precision, (C,E,G) filled circles: quadruple precision data from (B,D,E) in log-log plots and solid lines: fitting curves $r_{c} \sim N^{0.6}$, (parameters: $N_{E}=4 N_{I}$ and $N=N_{E}+N_{I}, K=100, \bar{v}_{E}=\bar{v}_{I}=1 \mathrm{~Hz}, J_{0}=1, \eta=0.9$, $\varepsilon=0.3, \tau_{\mathrm{m}}=10 \mathrm{~ms}$; averages of 10 runs with different network realizations). 

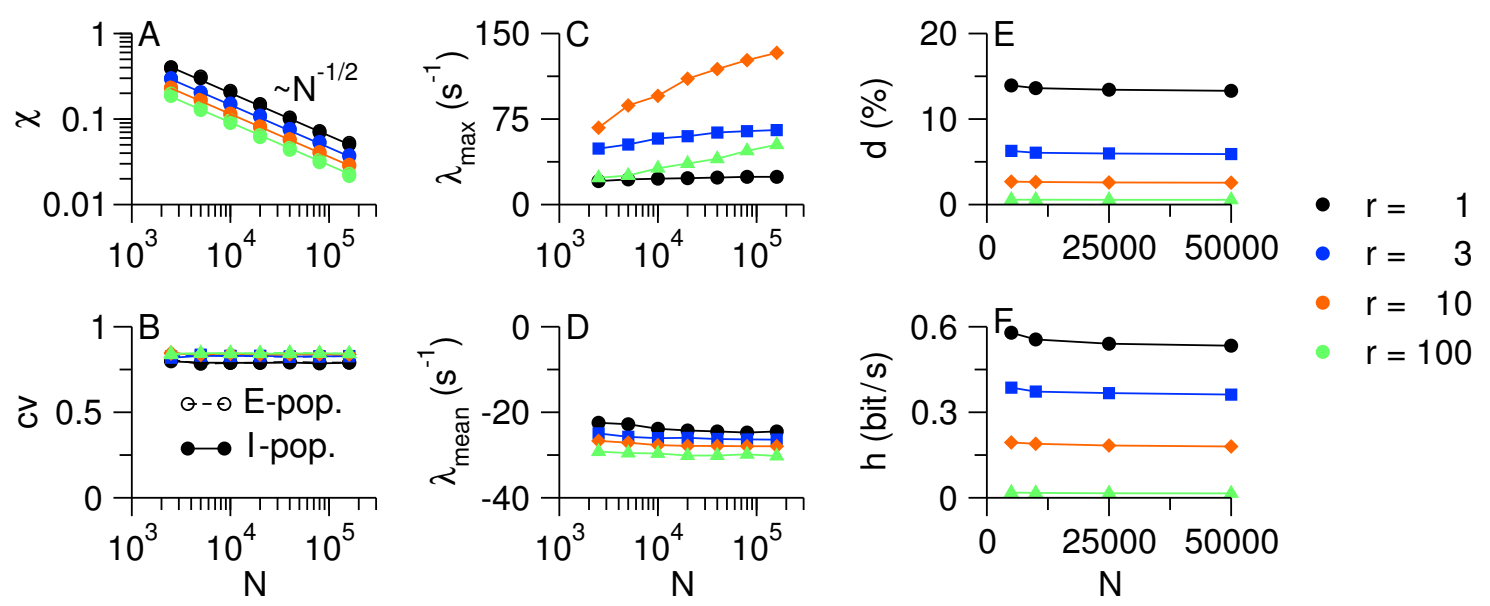

Figure 3.15 - Extensive chaos in excitatory-inhibitory balanced networks for varied network sizes $N$. (values of inhibitory networks as dotted lines for comparison) (A) Synchrony measure $\chi$ (straight line: $\chi \sim 1 / \sqrt{N}$ ), (B) average coefficient of variation cv, (C) largest Lyapunov exponent $\lambda_{\max }=\lambda_{1}$, (D) mean Lyapunov exponent $\lambda_{\text {mean }}=\frac{1}{N} \sum_{i} \lambda_{i}$, (E) attractor dimension in percent of phase space dimension $d=D / N$ and (F) entropy production rate per neuron $h=H / N,\left(N_{E}=4 N_{I}\right.$ and $N=N_{E}+N_{I}, K=100, \bar{v}_{E}=\bar{v}_{I}=1 \mathrm{~Hz}$, $J_{0}=1, \eta=0.9, \varepsilon=0.3, \tau_{\mathrm{m}}=10 \mathrm{~ms}$; averages of 10 runs with different network realizations).

critical AP onset rapidness $r_{c}$ for networks with an average firing rate $\bar{v}=1 \mathrm{~Hz}$ at excitatoryinhibitory feedback loop strength $\varepsilon \approx 0.3$ (Fig. 3.14A). For larger firing rates, $r_{c}$ also increased seemingly exponentially but stayed finite for larger excitatory-inhibitory feedback loop strength. Nevertheless, one could conclude that when the excitatory-inhibitory feedback loops are strong enough, these networks seem to generally exhibit chaotic dynamics. The scaling of the critical AP onset rapidness $r_{c}$ with the number of neurons was identical with that in exclusively inhibitory networks $r_{c} \sim N^{0.6}$. This is demonstrated for different average firing rates $\bar{v}$ and excitatory-inhibitory feedback loop strength $\varepsilon$ in Fig. 3.14B-G.

To complete the picture of the dynamics of excitatory-inhibitory networks, we examined the influence of the network size $N$ and number of incoming connections $K$ (Fig. 3.15, 3.16). This revealed a behavior qualitatively similar to inhibitory networks (dotted lines) as we have also observed in theta neuron networks. The synchrony measure $\chi$ decreased with $N^{-1 / 2}$ indicating asynchronous states. The coefficient of variation was constant and high indicating irregular firing activity. Although the largest Lyapunov exponent $\lambda_{\max }$ increased with $N$ and $K$, the relative attractor dimension $h=H / N$ and the average entropy production rate per neuron $h=H / N$ were constant. This reveals extensive chaos in excitatory-inhibitory networks and a well-defined thermodynamic limit.

We also observed the same behavior as in theta neuron networks, when increasing the number of synapses $K$ further. In dense networks of increasing size, the transition from the asynchronous irregular state to a synchronous irregular state was shifted to larger $K$ for $r=1$ (Fig. 3.17(a)). Because the transition is also shifted to larger $K$ for increasing AP onset rapidness, it was actually not visible for $r>1$ in the observed parameter range up to $N=200000$. In networks of fixed size, an increase of $K$ led to a gradual transition towards the state of a fully coupled network which was here reached at $K=2000$ (Fig. $\mathbf{3 . 1 7}(\mathbf{b})$ ).

So far, for low AP onset rapidness we have found similar extensive chaotic dynamics as in theta neuron networks in both inhibitory and excitatory-inhibitory networks. Increasing the AP onset rapidness, however, decreases the chaos in such balanced networks and can even lead to 

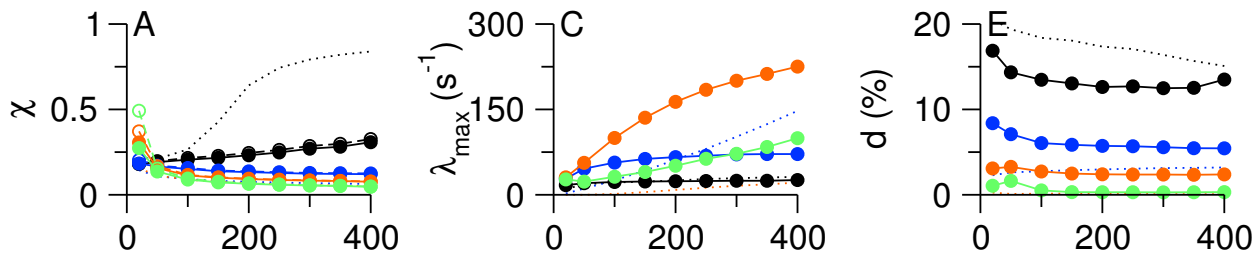

$\cdots \mathrm{N}_{\mathrm{E}}=0$
$\bullet \mathrm{r}=1$
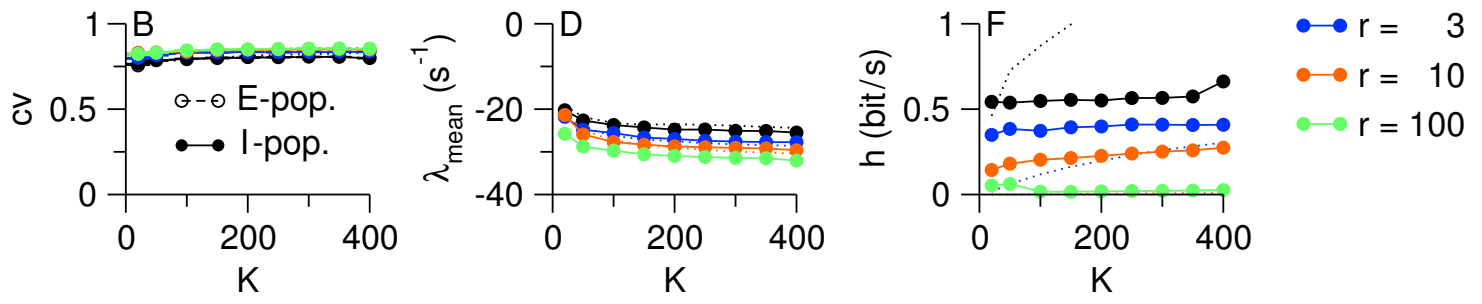

Figure 3.16 - Dynamics of excitatory-inhibitory balanced networks for different AP onset rapidness $r$ and varied number of synapses $K$. (values of inhibitory networks as dotted lines for comparison) (A) Synchrony measure $\chi,(B)$ average coefficient of variation cv, (C) largest Lyapunov exponent $\lambda_{\max }=\lambda_{1}$, (D) mean Lyapunov exponent $\lambda_{\text {mean }}=\frac{1}{N} \sum_{i} \lambda_{i}$, (E) attractor dimension in percent of phase space dimension $d=D / N$, (F) average entropy production rate per neuron $h=H / N$, (parameters: $N_{E}=4 N_{I}, N_{I}=20 K$, $\bar{v}_{E}=\bar{v}_{I}=1 \mathrm{~Hz}, J_{0}=1, \eta=0.9, \varepsilon=0.3, \tau_{\mathrm{m}}=10 \mathrm{~ms}$; averages of 10 runs with different network realizations for $K \leq 200$, for larger networks averages of 3 runs with different network realizations).
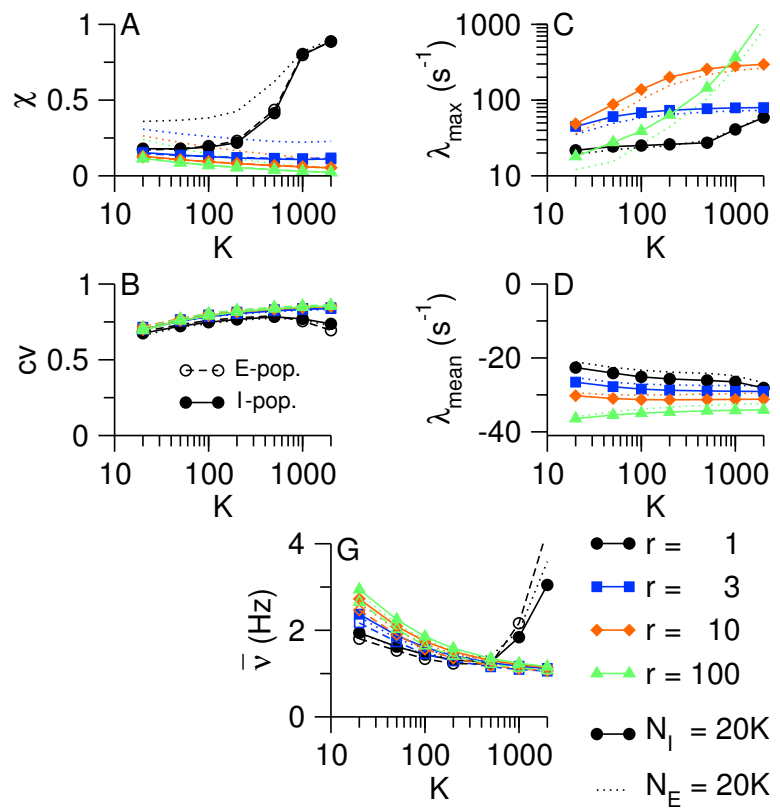

$\bullet r=1$

$\longrightarrow r=3$

$\leftrightarrow r=10$

$\leadsto r=100$

$\begin{aligned}-\mathrm{N}_{\mathrm{I}} & =20 \mathrm{~K} \\ \mathrm{~N}_{\mathrm{E}} & =20 \mathrm{~K}\end{aligned}$

(a) Dense networks with $N \propto K$.
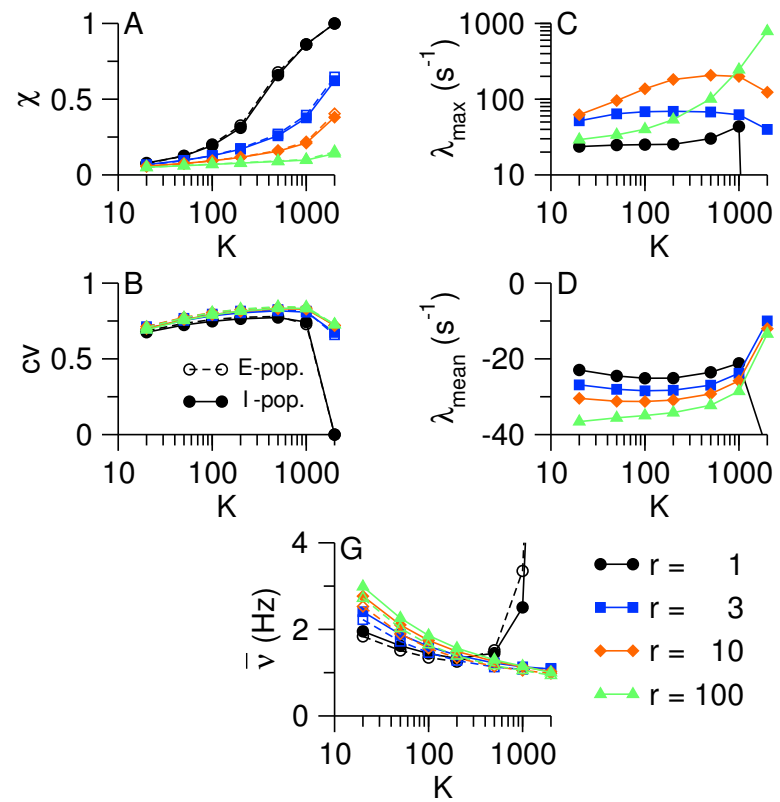

(b) Dense networks with $N_{E}=8000$ and $N_{I}=2000$ fixed.

Figure 3.17 - Occurrence of transition from asynchronous irregular to synchronous irregular state depends on AP rapidness $r$. (A) Synchrony measure , (B) average coefficient of variation, (C) largest Lyapunov exponent, (E) mean Lyapunov exponent, (F) actual network-averaged firing rate (parameters: $K=100, \bar{v}_{E}=\bar{v}_{I}=1 \mathrm{~Hz}, J_{0}=1, \eta=0.9, \varepsilon=0.3, \tau_{\mathrm{m}}=10 \mathrm{~ms}$; input currents chosen to fulfill balance equation (2.25) for target firing rate $\bar{v}_{\text {bal }}=1 \mathrm{~Hz}$ ). 


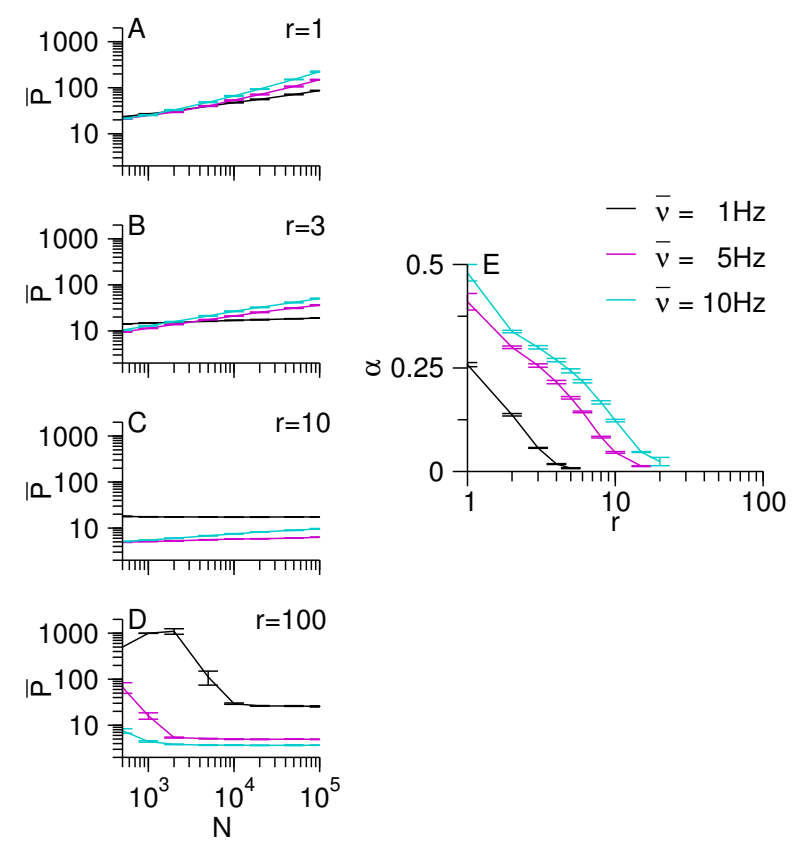

(a) Inhibitory networks.
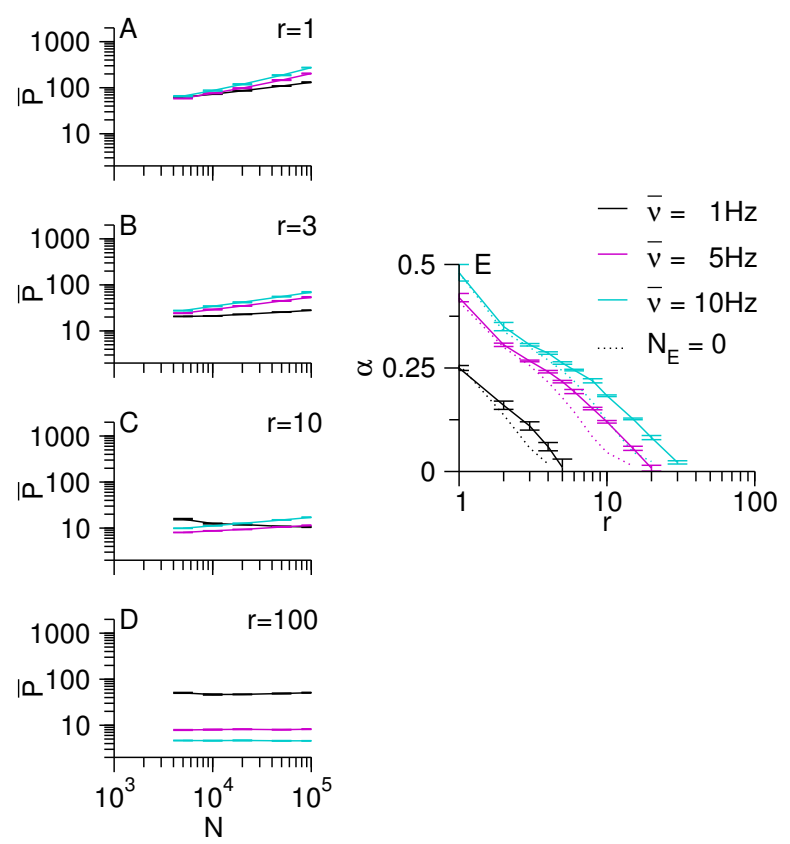

(b) Excitatory-inhibitory networks, dotted lines in (E) from inhibitory networks for comparison, $\left(N_{E}=4 N_{I}\right.$, $\left.\bar{v}_{E}=\bar{v}_{I}=\bar{v}, \eta=0.9, \varepsilon=0.3\right)$.

Figure 3.18 - Scaling of the average participation ratio in rapid theta neuron networks. (A-D) Average participation ratio $\bar{P}$ versus network size $N$ for different AP onset rapidness $r$, (E) power law scaling exponents $\alpha$ from fits $\bar{P} \sim N^{\alpha}$ to data in (A-D), (parameters: $K=100, J_{0}=1, \tau_{\mathrm{m}}=10 \mathrm{~ms}$ ).

a transition to stable dynamics. We called this the edge of chaos. What can we say about the spatiotemporal aspect in the chaotic regime.

In theta neuron networks, we have found a form of chaos called temporal network chaos with an interesting scaling regime of the fraction of neurons participating in the chaotic dynamics (Section 2.9. The number of neurons quantified by the average participation ratio $\bar{P}$ scaled sublinearly with the network size $N$. Although this meant that the fraction of neurons decreased algebraically with $N$, it differs from classical spatiotemporal chaos with strongly localized chaotic degrees of freedom for which $\bar{P}$ is expected to be independent of $N$.

The participation ratio was strongly affected by the AP onset rapidness (Fig. 3.18). With increasing AP onset rapidness starting from $r=1$, the power law exponent $\alpha$ of the scaling of the participation ratio $\bar{P} \sim N^{\alpha}$ decreased logarithmically and vanished at AP onset rapidness $r=\mathscr{O}(10)$. This coincides with the transition between the different regimes of the chaotic dynamics depending on the connectivity $K$ which is responsible for the peak in the largest Lyapunov exponent $\lambda_{\max }(r)$ as explained above (see Fig. 3.10). In the plateau regime $\left(\lambda_{\max }=\right.$ const) the participation ratio obeyed a power law scaling but the exponent decreased with increasing $r$ and was independent of the number of neurons in the increasing regime $\left(\lambda_{\max } \sim K\right)$. We would expect such a network size-independent participation ratio for localized chaotic degrees of freedom. This is the case in chaotic rapid theta neuron networks in both inhibitory and excitatory-inhibitory networks.

The different scaling of the average participation ratio in the two regimes below and above approximately $r=\mathscr{O}(10)$ confirms the observation of two qualitatively different chaotic dynamics depending on the AP onset rapidness of the single neurons. 


\subsection{Weak Pairwise Spike Correlations}

We have discussed in the introduction that pairwise spike correlations are expected to be weak and broadly distributed in the balanced state (Section 1.1), but this may still imply strongly correlated network states (Section 1.5). Therefore, we were wondering if pairwise correlations are indeed weak in balanced rapid theta neuron networks and what can be inferred about higher order correlations.

Renart et al. [32] and Schneidman et al. [78] use two different correlation measures to measure the pairwise spike correlations between two neurons $i$ and $j$. Renart et al. use the Pearson coefficient

$$
\rho_{i j}(\tau)=\frac{\operatorname{COV}\left(n_{i}(t), n_{j}(t+\tau)\right)}{\sqrt{\sigma_{i}^{2} \sigma_{j}^{2}}},
$$

where COV denotes the covariance and $\sigma^{2}$ the variance of the spike counts. Schneidman et al. use the correlation coefficient

$$
c_{i j}(\tau)=\frac{\operatorname{COV}\left(n_{i}(t), n_{j}(t+\tau)\right)}{\bar{v}_{i} \bar{v}_{j}} .
$$

An overview of different correlation measures used in the literature is provided in Appendix C. The difference between the Pearson and the correlation coefficient is the normalization of the spike correlations with the standard deviation of the neurons' firing rates or the average neurons' firing rates, respectively. Thus, the Pearson coefficient is bounded between $\rho_{i j} \in[0,1]$ and the correlation coefficient $c_{i j} \in[0, \infty]$. Usually, the spike counts are calculated by binning the time series, equivalent of using a box-kernel. We will calculate the spike counts using a Gauss-kernel. The width of the kernels must be chosen carefully and should be in the order of the correlation time in the networks (Appendix C). This is found to be in the order of $10 \mathrm{~ms}$ in the studied networks.

The correlation coefficients $c_{i j}(0)$ and the Pearson coefficients $\rho_{i j}(0)$ of 150 theta neurons and their histograms of inhibitory and excitatory-inhibitory networks are compared in Fig. 3.19. The 150 neurons are ordered in decreasing firing rate and grouped in three blocks. The first block consists of 50 neurons with average firing rates of $\pm 10 \%$ around the network-averaged rate plus one standard deviation of the rate distribution, the second block consists of 50 neurons with average firing rates of $\pm 10 \%$ around the network-average, and the third block consists of 47 neurons with average rates of $\pm 10 \%$ around the network-average rate minus one standard deviation. After each block is one additional random postsynaptic neuron. In the excitatory-inhibitory networks, excitatory neurons are grouped in the first part (80\%) and inhibitory neurons in the second part $(20 \%)$. It can be seen that the pairwise spike correlations are basically identical in both types of networks. In excitatory-inhibitory networks the correlations are furthermore identical between excitatory neurons, between inhibitory neurons and between excitatory and inhibitory neurons.

The temporal characteristics of the correlation coefficient $c(\tau)$ and the Pearson coefficients $\rho(\tau)$ of balanced inhibitory networks reveal a typical correlation time scale of $\tau_{c} \approx 10 \mathrm{~ms}$. Figure $\mathbf{3 . 2 0}$ presents the temporal spike correlations of the three blocks of neurons described above in inhibitory and excitatory-inhibitory networks. Theta neuron networks $(r=1)$ exhibit weak oscillatory spike correlations that indicate the proximity of these networks with $K=100$ to the transition to synchrony at $K_{c} \approx 200$. Networks of neurons with larger AP onset rapidness and leaky integrate and fire (LIF) neurons are not close to this transition for this parameter set as discussed above and therefore do not show this oscillation. In excitatory-inhibitory networks, the qualitative behavior of the pairwise spike correlations is similar to the one in inhibitory networks. The pairwise spike 

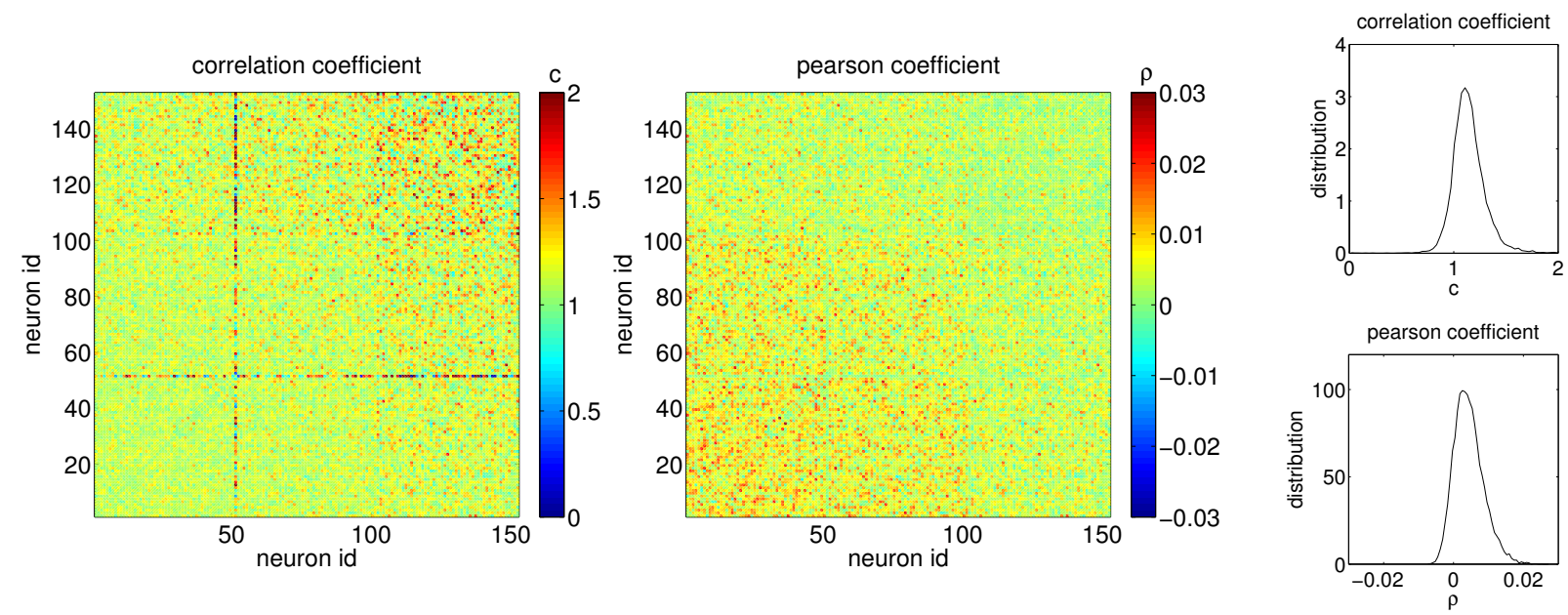

(a) Inhibitory networks.
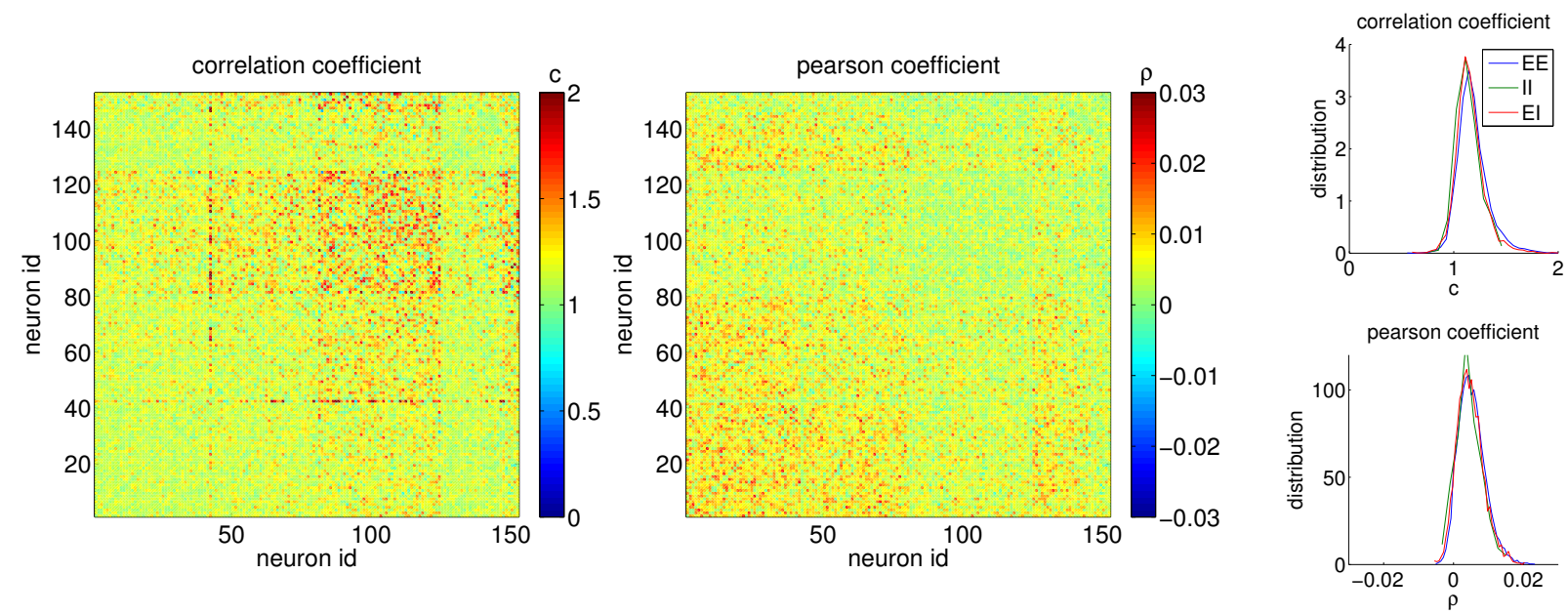

(b) Excitatory-inhibitory networks with $80 \%$ excitatory and $20 \%$ inhibitory neurons.

Figure 3.19 - Comparison of correlation coefficient, Eq. (3.23), and Pearson coefficient, Eq. (3.22), in theta neuron networks. The neurons are ordered with decreasing firing rates. The first block is composed of neurons with firing rates around the mean plus one standard deviation of the rate distribution in the network (from $1.8 \ldots 1.5 \mathrm{~Hz}$ ), the second of neurons around the mean firing rate (from $1.1 \ldots 0.92 \mathrm{~Hz}$ ) and the third of neurons around the mean minus one standard deviation (from $0.39 \ldots 0.32 \mathrm{~Hz}$ ). After each block comes one random postsynaptic neuron. In (b) the first $80 \%$ of the plot are excitatory neurons and the second $20 \%$ inhibitory neurons. (Length of time series is 10000 spikes per neuron (about $2.8 \mathrm{~h}$ ), the standard deviation of the Gauss-Kernels is $T=10 \mathrm{~ms}$, other parameters: $N_{I}=1000, N_{E}=4000, K=100, \bar{v}=1 \mathrm{~Hz}, J_{0}=1$, $\left.\tau_{\mathrm{m}}=10 \mathrm{~ms}, \eta=0.9, \varepsilon=0.3\right)$. 

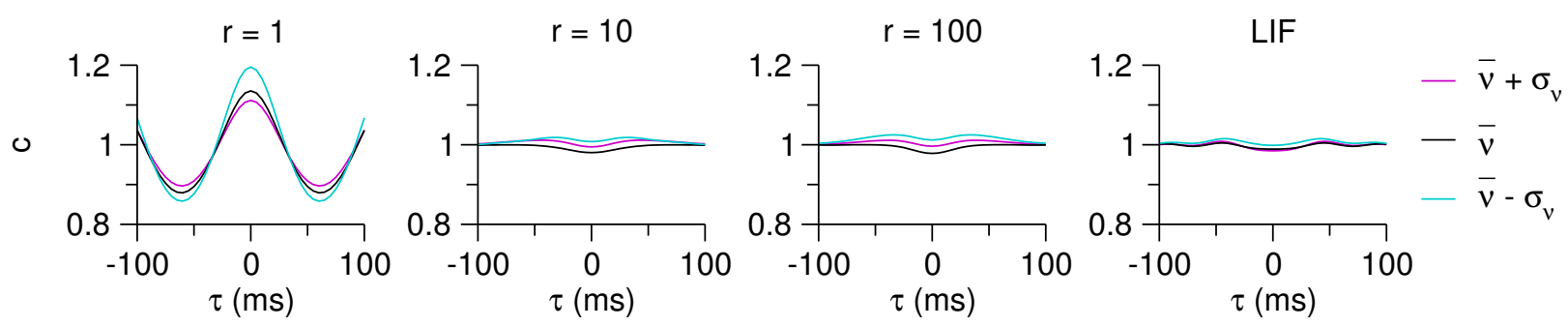

(a) Correlation coefficient in inhibitory networks.

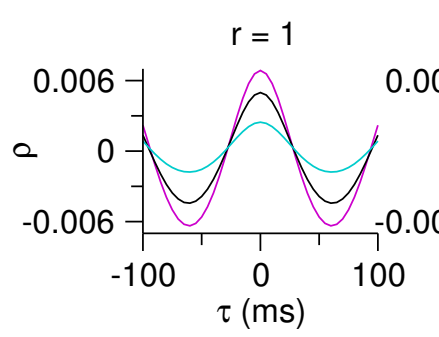

$$
r=10 \quad r=100
$$

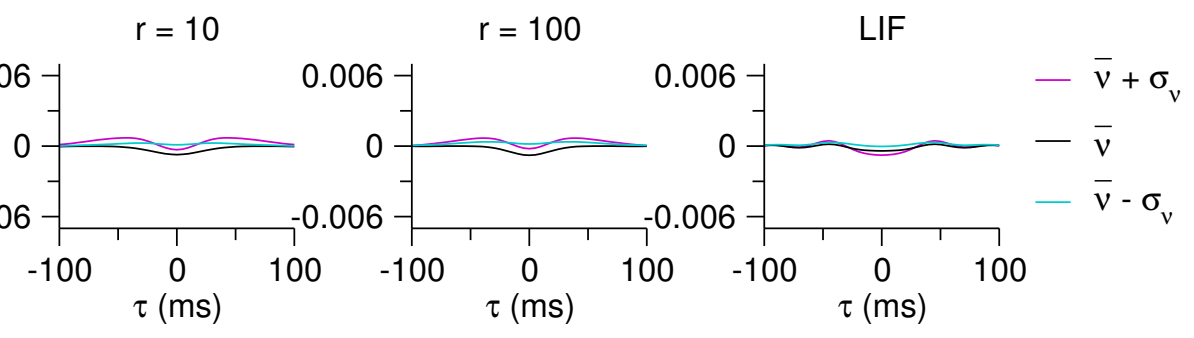

LIF

(b) Pearson coefficient in inhibitory networks.
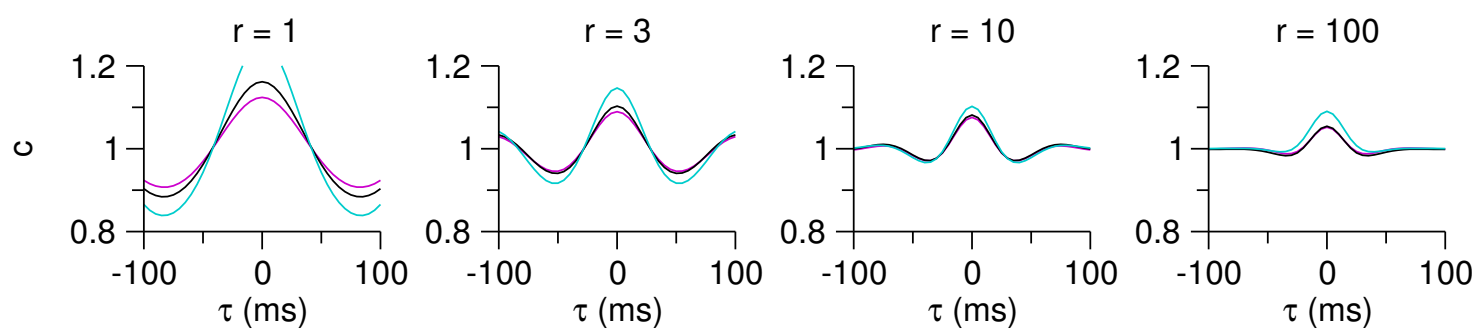

$-\bar{v}+\sigma_{v}$

$-\bar{v}$

$-\bar{v}-\sigma_{v}$

(c) Correlation coefficient in excitatory-inhibitory networks $\left(N_{E}=4000, \varepsilon=0.3, \eta=0.9\right)$.

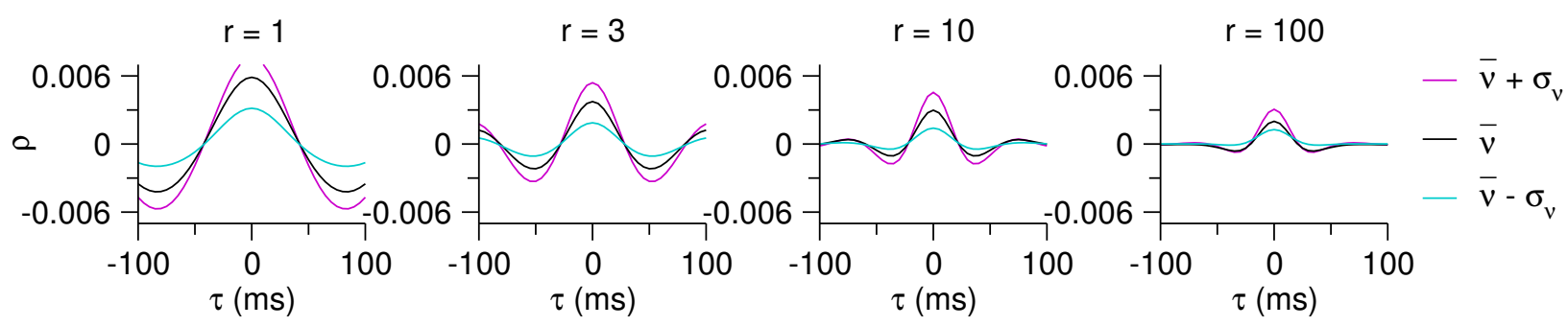

(d) Pearson coefficient in excitatory-inhibitory networks $\left(N_{E}=4000, \varepsilon=0.3, \eta=0.9\right)$.

Figure 3.20 - Averaged pairwise spike correlations of 50 neurons in three different firing rate intervals. (Length of time series is 10000 spikes per neuron (about 2.8h), the standard deviation of the Gauss-Kernels is $T=10 \mathrm{~ms}$, other parameters: $N=1000, K=100, \bar{v}=1 \mathrm{~Hz}, J_{0}=1, \tau_{\mathrm{m}}=10 \mathrm{~ms}$ ). 


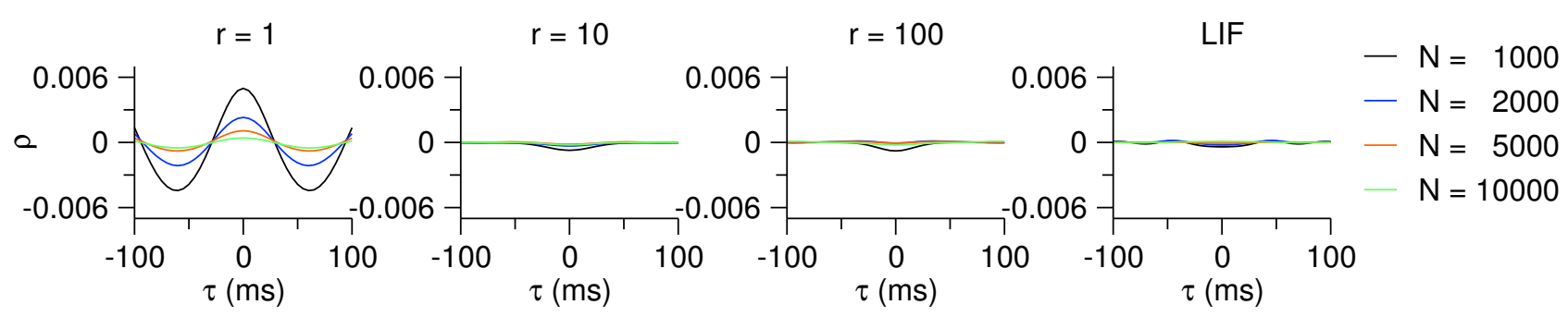

(a) Number of neurons $N$ in inhibitory networks varied.

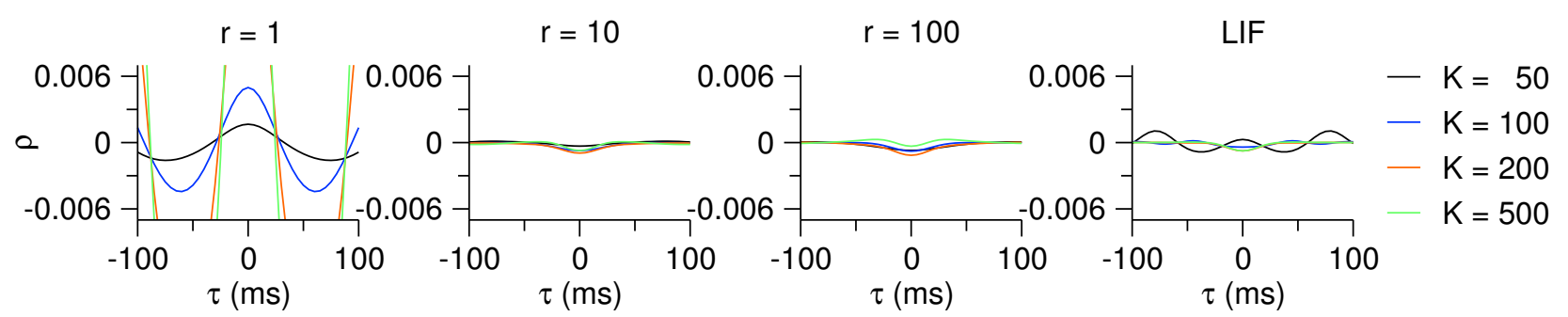

(b) Number of synapses $K$ in inhibitory networks varied.

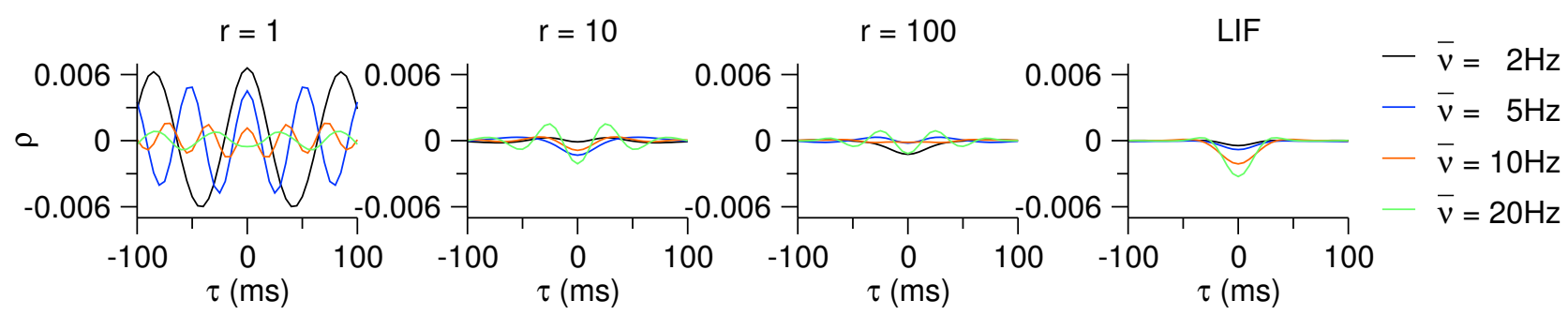

(c) Network-firing rate $\bar{v}$ in inhibitory networks varied.

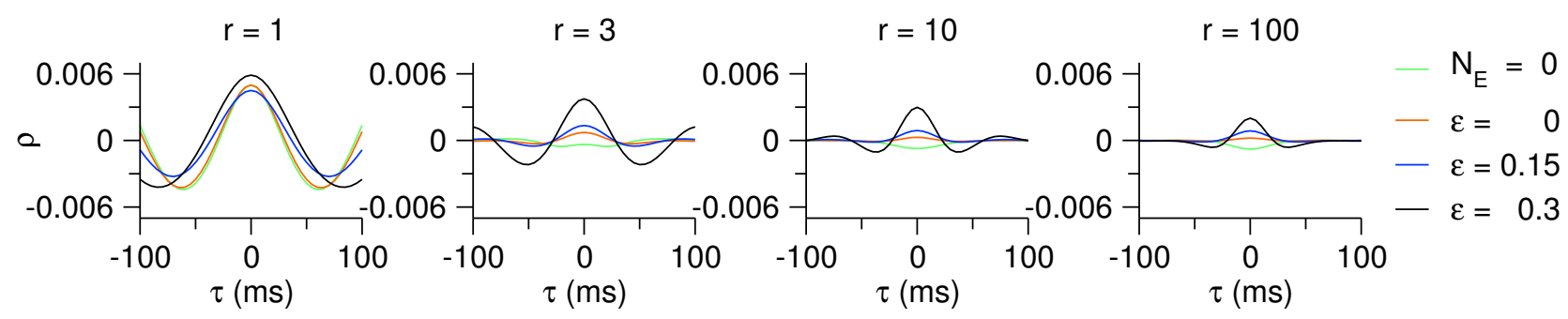

(d) Activation of excitatory-inhibitory feedback loops in excitatory-inhibitory networks with $\varepsilon$.

Figure 3.21 - Pairwise spike correlations of neurons with approximately the average firing rate in balanced inhibitory networks. (Length of time series is 10000 spikes per neuron (about $2.8 \mathrm{~h}$ ), the standard deviation of the Gauss-Kernels is $T=10 \mathrm{~ms}$, other parameters: $N=1000, K=100, \bar{v}=1 \mathrm{~Hz}, J_{0}=1$, $\tau_{\mathrm{m}}=10 \mathrm{~ms}$; in excitatory-inhibitory networks $\left.N_{E}=4000, N_{I}=1000, \eta=0.9\right)$. 


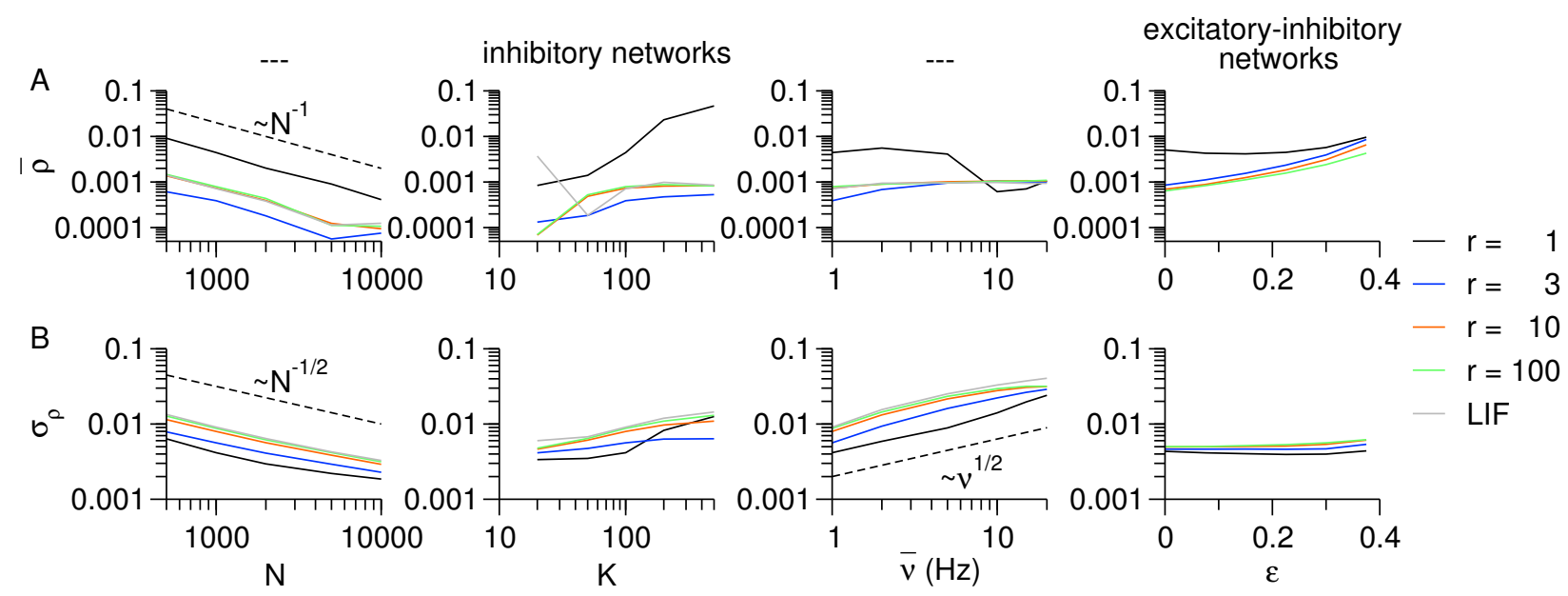

Figure 3.22 - Average and standard deviation of the distributions of Pearson coefficient of 150 randomly chosen neurons in rapid theta neuron networks. (A) absolute values of the averages $\bar{\rho}$ of the distributions of Pearson coefficients versus network size $N$ (dashed line: guide for the eye for $\bar{\rho} \sim 1 / N$ ), number of synapses per neuron $K$, network-averaged firing rate $\bar{v}$ and excitatory-inhibitory feedback loop strength $\varepsilon$, (B) standard deviation $\sigma_{\rho}$ of the distributions of Pearson coefficients (dashed lines: guide for the eye for $\sigma_{\rho} \sim 1 / \sqrt{N}$ and $\sigma_{\rho} \sim \sqrt{\bar{v}}$ ), (length of time series is 10000 spikes per neuron, the standard deviation of the Gauss-Kernels is $T=10 \mathrm{~ms}$, other parameters: $N_{I}=1000, K=100, \bar{v}=1 \mathrm{~Hz}, J_{0}=1, \tau_{\mathrm{m}}=10 \mathrm{~ms}$, in excitatory-inhibitory networks $N_{E}=4000, \eta=0.9$ ).

correlations are generally very weak in the studied networks and quite similar for neurons with different average firing rates. We can therefore continue to study the correlations of neurons around the network-averaged firing rate which is representative for all other neurons.

The pairwise spike correlations for the second block of neurons with firing rates around the network-averaged firing rate are displayed for various network parameters in Fig. 3.21. It can be seen that the magnitude of the pairwise correlations decreased with increasing network size $N$ (Fig. 3.21(a)). This is characteristic for the asynchronous state. The typical correlation time was independent of the network size about $\tau_{c} \approx 10 \mathrm{~ms}$. Increasing the connectivity $K$ increased the pairwise correlations in theta neuron networks as expected for the transition to synchrony but hardly affected the pairwise correlations in networks with larger AP onset rapidness (Fig. 3.21(b)). The typical correlation time was insensitive to the connectivity. An increase of the average firing rate $\bar{v}$ in the networks reduced the strength of pairwise correlations in theta neuron networks but increased the correlations in networks with larger AP onset rapidness (Fig. 3.21(c)). In excitatory-inhibitory networks, the pairwise correlations were hardly affected by the activation of excitatory-inhibitory feedback loops in theta neuron networks but generally increased with increased excitatory coupling in networks with larger AP onset rapidness (Fig. 3.21(d)). We also observe that the magnitude of the averaged pairwise correlations decreased with increased AP onset rapidness. The opposite was the case for the width of the distribution of pairwise correlations, as will become clear in the following. As a final remark on the temporal pairwise spike correlations: all studied networks exhibit the typical correlation time $\tau_{c} \approx 10 \mathrm{~ms}$ of the order of the membrane time constant, which justifies the used width of the Gauss-kernels of $T=10 \mathrm{~ms}$ in the calculation of the correlation coefficients.

The distributions of pairwise spike correlation of 150 randomly chosen neurons in inhibitory and excitatory-inhibitory networks are presented in Fig. 3.23 . The mean and width of the distribution are summarized in Fig. 3.22. As predicted for the asynchronous balanced state [32], the average pairwise correlation goes to zero for large networks as $\bar{\rho} \sim 1 / N$, but the width of the distribution, 

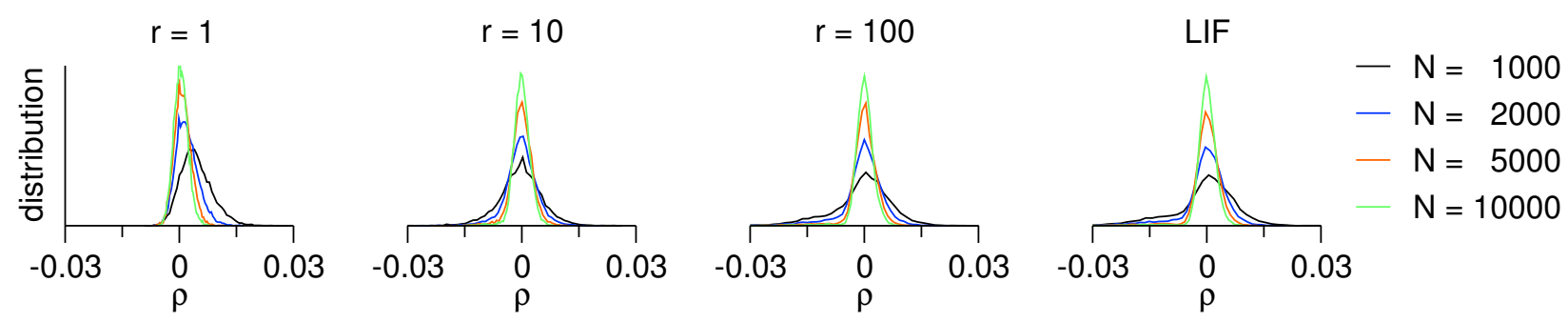

(a) Number of neurons $N$ in inhibitory networks varied.
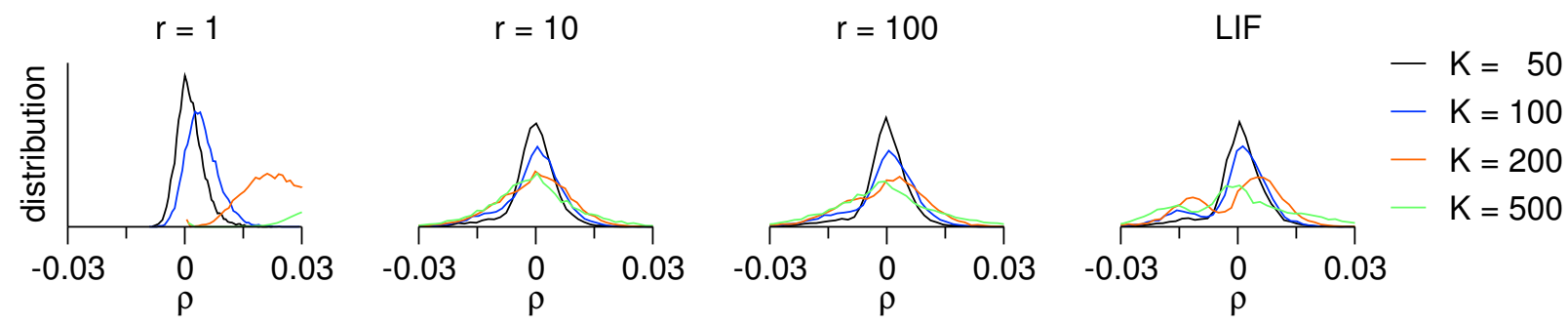

(b) Number of synapses $K$ in inhibitory networks varied.
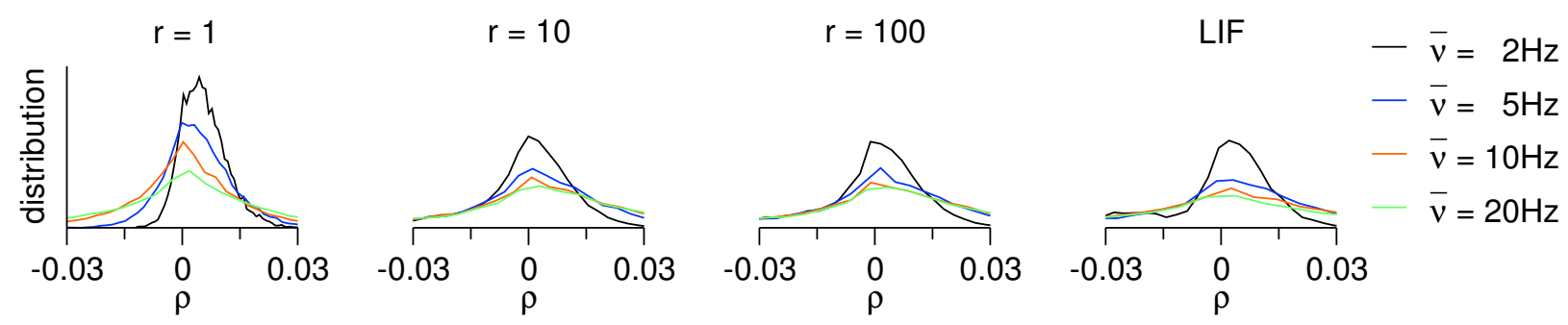

(c) Network-average firing rate $\bar{v}$ in inhibitory networks varied.
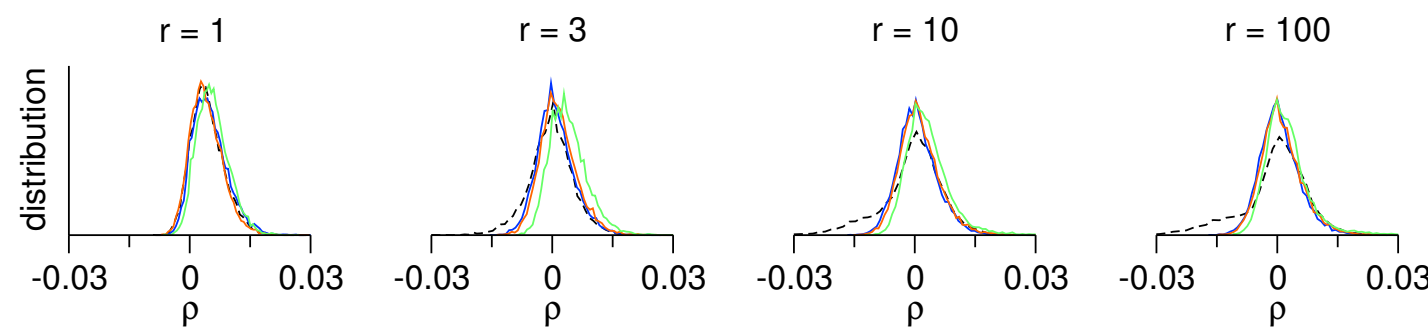

$$
\begin{aligned}
N_{E} & =0 \\
-\varepsilon & =0 \\
-\varepsilon & =0.15 \\
-\varepsilon & =0.3
\end{aligned}
$$

(d) Activation of excitatory-inhibitory feedback loops in excitatory-inhibitory networks with $\varepsilon$.

Figure 3.23 - Distribution of pairwise spike correlations of 150 randomly chosen neurons in balanced inhibitory networks. (Length of time series is 10000 spikes per neuron, the standard deviation of the Gauss-Kernels is $T=10 \mathrm{~ms}$, other parameters: $N=1000, K=100, \bar{v}=1 \mathrm{~Hz}, J_{0}=1, \tau_{\mathrm{m}}=10 \mathrm{~ms}$; in excitatory-inhibitory networks $N_{E}=4000, N_{I}=1000, \eta=0.9$ ). 
the standard deviation goes to zero as $\sigma_{\rho} \sim 1 / \sqrt{N}$, indicating broadly distributed pairwise correlations. One might speculate whether this fact of a broad distribution might imply higher order correlations. Another interesting observation is the increase of the width of the pairwise correlations with the square root of the network-averaged firing rate $\sigma_{\rho} \sim \sqrt{\bar{v}}$. In excitatory-inhibitory networks, the activation of the excitatory-inhibitory feedback loops with $\varepsilon$ slightly increased the average correlations but did not change the width of the distributions. Altogether, this analysis of the pairwise spike correlations confirmed the prediction of weak and broadly distributed pairwise spike correlations in the balanced state.

\subsection{Entangled Statistics}

Weak pairwise correlations do not necessarily imply vanishing higher order correlations (see Section 1.5). Can we deduce more about the strength of higher order correlation from our analysis? We have shown that rapid theta neuron networks undergo a transition from chaotic to stable dynamics at a parameter dependent critical AP onset rapidness $r_{c}$. Networks with chaotic dynamics for $r<r_{c}$ exhibit strange attractors with fractal dimensions $D>1$. Networks with stable dynamics for $r>r_{c}$ exhibit one-dimensional attractors $D=1$ which implies periodic orbits. One would expect that lower dimensional attractors imply strongly correlated network states, but the pairwise spike correlations were generally weak independent of this dramatic change of the collective networks dynamics.

In fact, the attractor dimension can be related to the highest order $n$ of nonvanishing correlations between $n$ neurons $C^{(n)}=\left\langle s_{i}(t) s_{j}\left(t^{\prime}\right) \ldots\right\rangle$. The first order correlations describe the average firing rates of the neurons, the second order the pairwise spike correlations and so on. In a network of $N$ independent or non-interacting neurons, the attractor dimension would be $D=N$. The independence means that there are no higher order correlations, thus $n=1$. In a stable periodic network state, the attractor dimension is $D=1$ and all neurons would be statistically dependent, thus $n=N$. If the network state was quasiperiodic with $x$ incommensurable frequencies, the attractor dimension would be $D=x$ and correlations of order $n \geq N-x+1$ would vanish. We can thus conjecture that generally correlations of higher order than $n=N-D+1$ vanish.

The decreasing attractor dimension for increasing AP onset rapidness (Fig. 3.13) thus implies that the neurons become more entangled for larger AP onset rapidness despite weak pairwise correlations. We will demonstrate this in an example of a small network for different values of AP onset rapidness $r$ (Fig. 3.24). Displayed are the spike trains of 10 out of 20 neurons and the Poincaré-sections of the phases of neuron 1 and 2 at times when neuron 3 spikes. The Poincarésections represent a cut through the attractor. All spike trains show the asynchronous and irregular firing activity in these networks. Below the critical AP onset rapidness $r_{c}=12$, the Poincarésections are a chaotic cloud. The dimensionality of the attractor is $D \approx 12.5$ for $r=1$ and stays $D \gtrsim 3$ up to the transition to stable dynamics at $r_{c}=12$. With $r=12$, one can see that the firing pattern of the entire network becomes periodic as expected in the regime of stable dynamics. The Poincaré- sections for $r=12$ and $r=100$ display a cut through the one-dimensional attractors, thus dots. The number of dots represents the period length which is larger than one. We can thus conclude that despite weak pairwise spike correlations and asynchronous firing of the neurons, the statistics or neural networks becomes more entangled with higher AP onset rapidness.

The period length indicated by the number of dots in the Poincaré-sections for $r>r_{c}$ increased exponentially with the number of neurons, together with the transient lengths to the periodic states. This characteristic of balanced networks with very fast AP onset rapidness is similar to leaky 

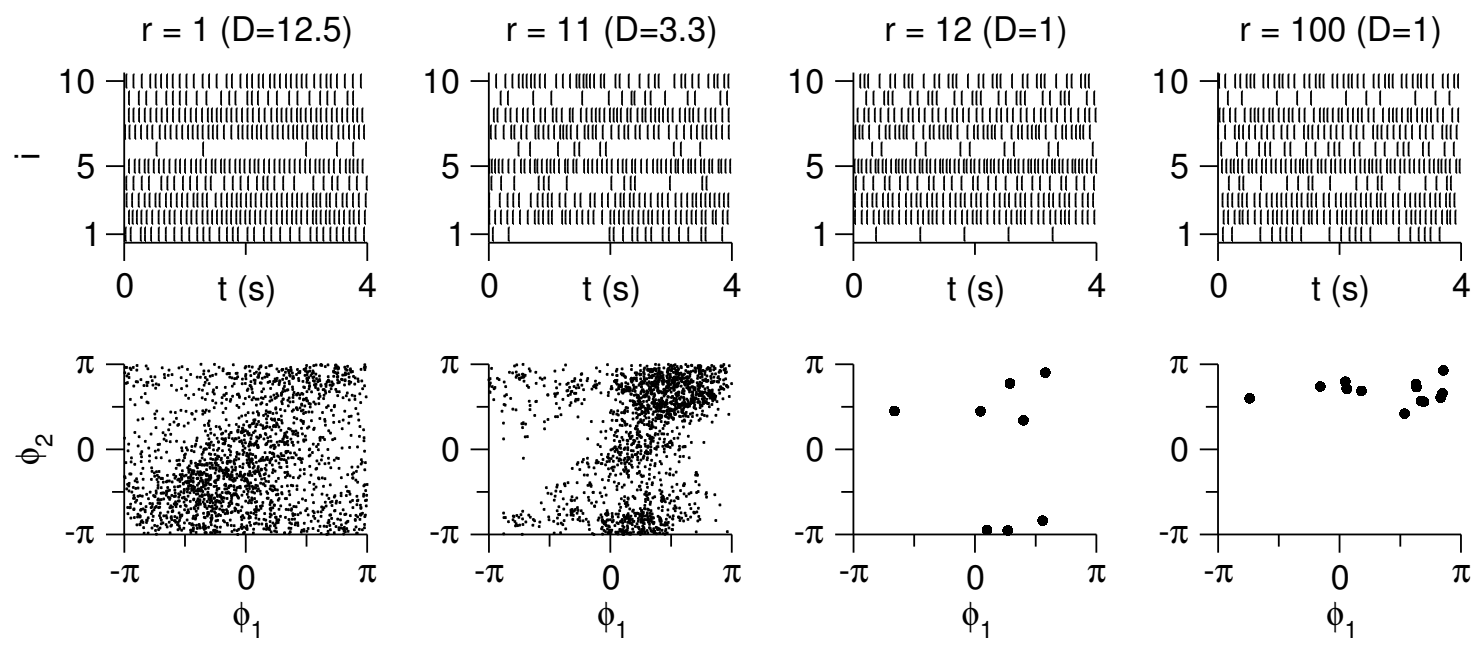

Figure 3.24 - Entangled statistics in rapid theta neuron networks. Upper panels spike trains of 10 randomly chosen neurons, lower panels: Poincaré-sections of the phases of neurons 1 and 2 when neuron 3 emits a spike, the AP onset rapidness $r$ was increased from left to right in otherwise identical networks, (parameters: $N=20, K=10, \bar{v}=10 \mathrm{~Hz}, J_{0}=1, \tau_{\mathrm{m}}=10 \mathrm{~ms}$; data from 100 spikes per neuron on average).

integrate and fire networks and should be investigated further for a thorough understanding of the balanced state in the stable regime. 


\subsection{Summary}

In this chapter we investigated the influence of the action potential (AP) onset rapidness of the individual neurons on the collective network dynamics. A new exactly solvable neuron model with variable AP onset rapidness, called the rapid theta neuron model, allowed for the direct application of the approach to study neural network dynamics introduced in Chapter 2 . The presented results show that the AP onset rapidness strongly affects the collective network dynamics.

Networks of rapid theta neurons in the balanced state undergo a phase transition from chaotic to stable dynamics at a parameter-dependent critical AP onset rapidness $r_{c}$. We call this the edge of chaos. The general properties of the balanced state such as the macroscopic firing statistics and the weak pairwise spike correlations are basically independent of the AP onset rapidness and fail to capture the dramatic change of the underlying network dynamics.

The AP onset rapidness also influences the dynamics within the chaotic regime qualitatively. We have observed two different dynamic regimes indicated by a peak in the largest Lyapunov exponent with respect to the AP onset rapidness $r$. For low $r<10$, the network dynamics is very similar to the dynamics of theta neurons $(r=1)$ studied in Chapter 2 . For large $r>10$, the network dynamics shows qualitative differences to the dynamics of theta neurons. Both phase transitions to a synchronous state disappear and the intensity of the chaos decreases leading to the edge of chaos and stable dynamics for very large $r$. Because we expect cortical neurons to exhibit such a large AP onset rapidness, we can conclude that the collective dynamics of cortical neurons qualitatively differs from the collective dynamics of neurons with low AP onset rapidness.

The network dynamics of rapid theta neurons is characterized by:

- Deterministic chaos, characterized by positive and finite Lyapunov exponents. A peak in the largest Lyapunov exponent for increasing AP onset rapidness indicates a qualitative change of the network dynamics.

- Extensive chaos, characterized by network size-invariant Lyapunov spectra and a linear increase of the number of positive Lyapunov exponents, attractor dimension and entropy production rate with the number of neurons.

- Monotonously decreasing attractor dimension with increasing AP onset rapidness, implying entangled statistics towards the edge of chaos despite generally weak pairwise spike correlations in the balanced state.

- Monotonously decreasing entropy production rate with increasing AP onset rapidness, implying reduced loss of information in cortical networks through larger AP onset rapidness of the single neurons.

For very large AP onset rapidness, the AP initiation becomes basically instantaneous similar to the leaky integrate and fire model. Our results demonstrate that the characterization of the dynamics of inhibitory networks in the limit of instantaneous AP initiation $(r \rightarrow \infty)$ depends on the order in which this limit and the large system limit are taken. It can either be characterized as extremely chaotic $\left(\lambda_{\max }=\infty\right)$ or stable $\left(\lambda_{\max }=0\right)$. Such inhibitory networks of leaky integrate and fire neurons were previously shown to exhibit stable chaos [4-7]. How stable this form of irregular dynamics is with respect to finite perturbations and temporally extended synaptic transmission will be the topic of the next two chapters. 


\section{Networks of Correlated Leaky Integrate and Fire Neurons Temporal Synaptic Transmission}

The approach presented in Chapter 2 for the characterization and quantification of neural network dynamics can be applied to a wide class of neuron models and network topologies. In this chapter, we introduce an extension to incorporate temporal postsynaptic currents. Whereas the synaptic coupling was composed of $\delta$ pulses so far, we will here use exponentially decaying postsynaptic currents. The incorporation of such biologically more realistic synaptic waveforms yields further insight into the dynamics of real neural networks. We will develop this extension for networks of leaky integrate and fire (LIF) neurons.

Previously, randomly coupled LIF networks with exclusively inhibitory neurons and $\delta$ pulse coupling were shown to exhibit stable dynamics with respect to infinitesimal perturbations [4-7]. An analysis of up to the first three Lyapunov exponents of LIF networks with exponentially decaying synaptic pulses recently revealed that such networks can exhibit chaotic dynamics [5]. The authors of this study also showed that whether the dynamics of these networks is stable or chaotic depends on the the synaptic time constant, the temporal delay and the connectivity. To develop chaotic dynamics these networks require a high connectivity, and considerably large synaptic time constants with respect to the delay time [5].

The extension of the approach developed in Chapter 2 to LIF networks with exponentially decaying synaptic pulses allows for the calculation of the complete Lyapunov spectra, which yields additional information beyond the first Lyapunov exponents. The derivation of the attractor dimension and the dynamical entropy production rate in such networks provide a detailed quantification of the chaotic dynamics. The comparison of these results with the ones from the previous chapters allow us to relate the effect on the information loss due to the incorporation of temporal synaptic transmissions to the effect of the incorporation of a dynamic AP generation in the single neurons.

\subsection{Correlated Leaky Integrate and Fire Model}

We here consider the same random topology as in Chapter 2 and 3 with the difference of leaky integrate and fire neurons which are coupled with exponentially decaying synaptic currents (Fig. 4.1). Because the temporal synaptic currents induce temporal current correlations, the model is called correlated leaky integrate and fire (cLIF) model. 

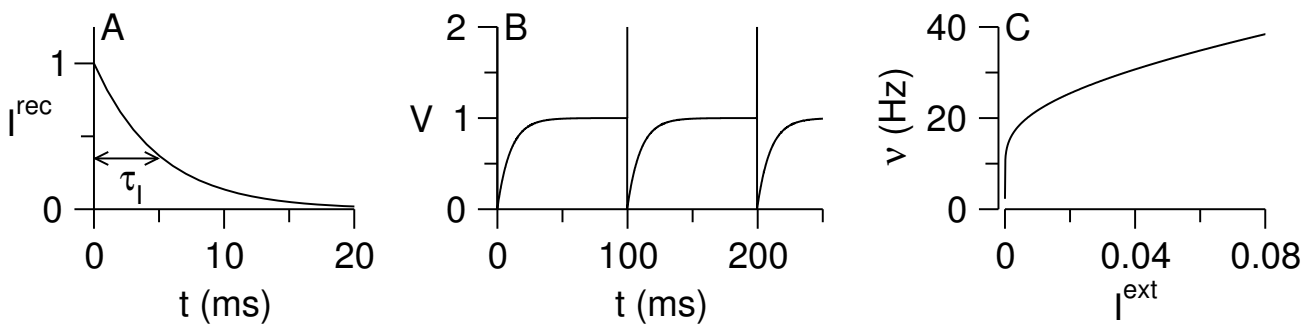

Figure 4.1 - The correlated leaky integrate and fire model. (A) Exponentially decaying postsynaptic currents with time constant $\tau_{\mathrm{I}}$, (B) voltage trace of a leaky integrate and fire model, (C) gain function (FIcurve) $v=1 /\left(\tau_{\mathrm{m}} \log \left(1+1 / I^{\text {ext }}\right)\right)$ of a leaky integrate and fire neuron for suprathreshold input current $I^{\text {ext }}$.

The $N$ leaky integrate and fire (LIF) neurons in the networks are described by their voltages $V_{i}$ with $i=1 \ldots N$, obeying the differential equation (2.1) with the linear term $F\left(V_{i}\right)=-V_{i}$, thus

$$
\tau_{\mathrm{m}} \frac{\mathrm{d} V_{i}(t)}{\mathrm{d} t}=-V_{i}(t)+I_{i}(t)
$$

This is complemented with a reset to $V_{R} \equiv 0$ whenever the voltage crosses the threshold $V_{T} \equiv 1$. At the time $t_{i}^{(s)}$ of the threshold crossing, a spike is said to be emitted and sent to the postsynaptic neurons. Instead of $\delta$ pulses, the postsynaptic neurons receive exponentially decaying currents. The equation of the synaptic input currents (2.2) thus reads

$$
I_{i}(t)=I_{T}+I_{i}^{\mathrm{ext}}+\sum_{j \in \operatorname{pre}(i)} \sum_{s} J_{i j} \frac{\tau_{\mathrm{m}}}{\tau_{\mathrm{I}}} \exp \left(-\frac{t-t_{j}^{(s)}}{\tau_{\mathrm{I}}}\right) \Theta\left(t-t_{j}^{(s)}\right) .
$$

The synaptic time constant is denoted $\tau_{\mathrm{I}}$, whereas the membrane time constant is $\tau_{\mathrm{m}}$ as before. $\Theta(t)$ denotes the Heaviside step function.

The exponentially decaying currents are normalized with the factor $\gamma=\tau_{\mathrm{m}} / \tau_{\mathrm{I}}$ such that the integrated postsynaptic current for one input is equivalent to the case of $\delta$ pulse coupling:

$$
J_{i j} \int \frac{\tau_{\mathrm{m}}}{\tau_{\mathrm{I}}} \exp \left(-\frac{t}{\tau_{\mathrm{I}}}\right)=J_{i j} \tau_{\mathrm{m}}
$$

To investigate the dynamics of the balanced state, the external currents and the synaptic coupling strengths exhibit the same scaling relation with the average number of synapses $K$ as before: $I_{i}^{\text {ext }} \mapsto$ $\sqrt{K} I_{i}^{\text {ext }}$ and $J_{i j} \mapsto-J_{i j} / \sqrt{K}$. Then the average input to the neurons in the asynchronous state leads to the balance equations derived for inhibitory and excitatory-inhibitory networks, Eq. 2.19) and (2.25), respectively.

The variance of the synaptic inputs depends on the synaptic time constant $\tau_{\mathrm{I}}$. Analogously to Eq. (2.20), the input current auto-correlation in balanced inhibitory networks is

$$
\begin{aligned}
C\left(t^{\prime}\right) & =\left\langle\delta I(t) \delta I\left(t+t^{\prime}\right)\right\rangle_{t} \\
& \approx\left(\frac{J_{0} \tau_{\mathrm{m}}}{\sqrt{K} \tau_{\mathrm{I}}}\right)^{2} K \bar{v} \int_{0}^{\infty} \exp \left(-\frac{t}{\tau_{\mathrm{I}}}\right) \exp \left(-\frac{t+t^{\prime}}{\tau_{\mathrm{I}}}\right) \mathrm{d} t \\
& =J_{0}^{2} \bar{v} \frac{\tau_{\mathrm{m}}^{2}}{2 \tau_{\mathrm{I}}} \exp \left(-\frac{t^{\prime}}{\tau_{\mathrm{I}}}\right) .
\end{aligned}
$$


This shows that the currents are temporally correlated with a magnitude $\sigma^{2}=J_{0}^{2} \bar{v} \tau_{\mathrm{m}}^{2} /\left(2 \tau_{\mathrm{I}}\right)$. In the case that will be studied mostly in the next sections $\left(\tau_{\mathrm{I}}=\tau_{\mathrm{m}} / 2\right)$ the magnitude of input fluctuations is identical to those in $\delta$ pulse coupled networks discussed in the previous chapters.

To yield the same magnitude of input fluctuations as in $\delta$ pulse coupled networks for any synaptic time constant $\tau_{\mathrm{I}}$, the postsynaptic currents (4.4) should be normalized with $\sqrt{2 \tau_{\mathrm{m}} / \tau_{\mathrm{I}}}$ instead of $\tau_{\mathrm{m}} / \tau_{\mathrm{I}}$. The problem then would be that the integrated current per input would not be normalized and the balance equation (2.19), thus the average network firing rate would depend on the synaptic time constants $\bar{v}=I_{0} /\left(J_{0} \sqrt{2 \tau_{\mathrm{m}} \tau_{\mathrm{I}}}\right)$. Again, in the case $\tau_{\mathrm{I}}=\tau_{\mathrm{m}} / 2$, this would yield the same equation as for $\delta$ pulse coupled networks. However, we decided to use the normalization factor $\tau_{\mathrm{m}} / \tau_{\mathrm{I}}$

\subsection{Iterative Map}

In the cLIF model, the iterative map introduced in Section 2.2 becomes two dimensional. The states of the neurons are described by $\vec{x}_{i}(t)=\left(V_{i}(t), I_{i}(t)\right)$. Both the voltage and the synaptic input currents are time-dependent quantities in the entire iteration of the map $f\left(\vec{x}_{i}(t)\right)$.

For clarity in the derivation of the iterative map, we will separate the synaptic input current (4.2) into the constant external currents $I_{i}^{\mathrm{c}}=I_{T}+\sqrt{K} I_{i}^{\text {ext }}$ (with $I_{T} \equiv V_{T} \equiv 1$ ) and the recurrent currents $I_{i}^{\text {rec }}(t)$, such that

$$
I_{i}(t)=I_{i}^{\mathrm{c}}+I_{i}^{\mathrm{rec}}(t)
$$

The evolution of the recurrent current can be written down in a differential equation:

$$
\tau_{\mathrm{I}} \frac{\mathrm{d} I_{i}^{\mathrm{rec}}(t)}{\mathrm{d} t}=-I_{i}^{\mathrm{rec}}(t)+\sum_{j \in \operatorname{pre}(i)} \sum_{s} \frac{J_{i j}}{\sqrt{K}} \tau_{\mathrm{m}} \delta\left(t-t_{j}^{(s)}\right) .
$$

The solution yields the evolution of the recurrent current between two successive spikes $t_{s}$ and $t_{s+1}$ in the network:

$$
f\left(I_{i}^{\mathrm{rec}}\left(t_{s}\right), t_{s+1}-t_{s}\right)=I_{i}^{\mathrm{rec}}\left(t_{s}\right) \exp \left(-\frac{t_{s+1}-t_{s}}{\tau_{\mathrm{I}}}\right) .
$$

If neuron $i^{*} \in \operatorname{post}\left(j^{*}\right)$ receives a pulse from the spiking neuron $j^{*}$ in this interval at $t_{s+1}$, the recurrent current is updated with

$$
g\left(I_{i^{*}}^{\mathrm{rec}}\left(t_{s+1}^{-}\right)\right)=I_{i^{*}}^{\mathrm{rec}}\left(t_{s+1}^{-}\right)+\frac{J_{i^{*} j^{*}}}{\sqrt{K}} \frac{\tau_{\mathrm{m}}}{\tau_{\mathrm{I}}} .
$$

The voltage $V_{i^{*}}(\mathrm{t})$ does not change immediately when a spike is received

$$
g\left(V_{i^{*}}\left(t_{s+1}^{-}\right)\right)=V_{i^{*}}\left(t_{s+1}^{-}\right) .
$$

The evolution of the voltage between two spikes is derived from the solution of Eq. (4.1) and the synaptic input (4.2) rewritten as $I_{i}(t)=I_{i}^{\mathrm{c}}+I_{i}^{\mathrm{rec}}(t)$. The inhomogeneous differential equation (4.1) is solved using the propagator method (see Appendix $(\mathrm{E})$ ). 


$$
\begin{aligned}
f\left(V_{i}\left(t_{s}\right), t_{s+1}-t_{s}\right) & =\exp \left(-\frac{t_{s+1}-t_{s}}{\tau_{\mathrm{m}}}\right) V_{i}\left(t_{s}\right)+\frac{1}{\tau_{\mathrm{m}}} \int_{t_{s}}^{t_{s+1}} \exp \left(-\frac{t_{s+1}-t^{\prime}}{\tau_{\mathrm{m}}}\right)\left(I_{i}^{\mathrm{c}}+I_{i}^{\mathrm{rec}}\left(t^{\prime}\right)\right) \mathrm{d} t^{\prime} \\
& =\exp \left(-\frac{t_{s+1}-t_{s}}{\tau_{\mathrm{m}}}\right) V_{i}\left(t_{s}\right)+\left[\exp \left(-\frac{t_{s+1}-t}{\tau_{\mathrm{m}}}\right) I_{i}^{\mathrm{c}}\right]_{t_{s}}^{t_{s+1}}+ \\
& =I_{i}^{\mathrm{c}}-\left(I_{i}^{\mathrm{c}}-V_{i}\left(t_{s}\right)\right) \mathrm{e}^{-\triangle t_{s}}+\exp \left(-\frac{t_{s+1}-t^{\prime}}{\tau_{\mathrm{m}}}\right) I_{i}^{\mathrm{rec}}\left(t_{s}\right) \exp \left(-\frac{t^{\prime}-t_{s}}{\tau_{\mathrm{I}}}\right) \mathrm{d} t^{\prime} \\
& \left.=I_{i}^{\mathrm{c}}-\left(I_{i}^{\mathrm{c}}-V_{i}\left(t_{s}\right)\right) \mathrm{e}^{-\triangle t_{s}}+\frac{t_{s+1}-\gamma t_{s}}{\tau_{\mathrm{m}}}\right) \frac{1}{\tau_{\mathrm{m}}} \int_{t_{s}}^{t_{s+1}} \exp \left(\frac{t^{\prime}}{\tau_{\mathrm{m}}}(1-\gamma)\right) \mathrm{d} t^{\prime} \\
& I_{i}^{\mathrm{rec}}\left(t_{s}\right) \\
1-\gamma & \exp \left(-\frac{t_{s+1}-\gamma t_{s}}{\tau_{\mathrm{m}}}\right)\left[\exp \left(\frac{t}{\tau_{\mathrm{m}}}(1-\gamma)\right)\right]_{t_{s}}^{t_{s+1}} \\
& =I_{i}^{\mathrm{c}}-\left(I_{i}^{\mathrm{c}}-V_{i}\left(t_{s}\right)\right) \mathrm{e}^{-\triangle t_{s}}+\frac{I_{i}^{\mathrm{rec}}\left(t_{s}\right)}{1-\gamma}\left(\mathrm{e}^{-\gamma \triangle t_{s}}-\mathrm{e}^{-\triangle t_{s}}\right) \\
& =I_{i}^{\mathrm{c}}+\frac{I_{i}^{\mathrm{rec}}\left(t_{s}\right)}{1-\gamma} \mathrm{e}^{-\gamma \triangle t_{s}}-\left(I_{i}^{\mathrm{c}}+\frac{I_{i}^{\mathrm{rec}}\left(t_{s}\right)}{1-\gamma}-V_{i}\left(t_{s}\right)\right) \mathrm{e}^{-\triangle t_{s}}
\end{aligned}
$$

with the abbreviations $\triangle t_{s}=\frac{t_{s+1}-t_{s}}{\tau_{\mathrm{m}}}$ and $\gamma=\frac{\tau_{\mathrm{m}}}{\tau_{\mathrm{I}}}$. Equations (4.5)-4.8) define the map (2.3) that iterates the neurons states $\vec{x}_{i}(t)=\left(V_{i}(t), I_{i}(t)\right)$ between successive spikes $\left\{t_{s}\right\}$ in the networks:

$$
\vec{x}_{i}\left(t_{s+1}\right)= \begin{cases}f\left(\vec{x}_{i}\left(t_{s}\right), t_{s+1}-t_{s}\right) & \text { if } i \notin \operatorname{post}\left(j^{*}\right) \\ g\left(f\left(\vec{x}_{i}\left(t_{s}\right), t_{s+1}-t_{s}\right)\right) & \text { if } i \in \operatorname{post}\left(j^{*}\right) .\end{cases}
$$

For true event-based simulations it is necessary to be able to calculate the next spike times analytically. Eq. (4.8) can be solved for the next spike time for 4 different ratios of the time constants $\gamma=\frac{1}{3}, \frac{1}{2}, 2,3$. In theses cases, the known expressions of the roots of quadratic and cubic equations yield closed form expressions for the next spike times, which completes the iterative map, Eq. 2.3. This is shown exemplary for the ratio $\gamma=2$ next.

\subsubsection{Example of Fast Synapses}

Fast synapses with a synaptic time constant of half the membrane time constant $\left(\tau_{\mathrm{I}}=\tau_{\mathrm{m}} / 2\right)$ are one of the special cases in which the voltage evolution function (4.8) can be inverted to yield an exact expression of the next spike time of the neuron. Neuron $i$ is said to emit a spike when its voltage crosses the threshold $V_{T}$. The time to the next spike $\triangle t_{i}$ from time $t$, given the neuron's state $\vec{x}_{i}(t)$ and $\gamma=2$ is thus implicitly defined by requiring:

$$
\begin{aligned}
f\left(V_{i}(t), \triangle t_{i}\right)=V_{T} & =I_{i}^{\mathrm{c}}+\frac{I_{i}^{\mathrm{rec}}(t)}{1-\gamma} \mathrm{e}^{-\gamma \triangle t_{i}}-\left(I_{i}^{\mathrm{c}}+\frac{I_{i}^{\mathrm{rec}}(t)}{1-\gamma}-V_{i}(t)\right) \mathrm{e}^{-\triangle t_{i}} \\
0 & =-I_{i}^{\mathrm{rec}}(t) \mathrm{e}^{-2 \triangle t_{i}}-\left(I_{i}^{\mathrm{c}}-I_{i}^{\mathrm{rec}}(t)-V_{i}(t)\right) \mathrm{e}^{-\triangle t_{i}}+I_{i}^{\mathrm{c}}-V_{T} .
\end{aligned}
$$


Abbreviating $T_{i}=\mathrm{e}^{-\triangle t_{i}}$, the roots of Eq. (4.9) can be obtained from the standard solution of a quadratic equation $0=a T_{i}^{2}+b T_{i}+c$. Identifying this standard quadratic equation with Eq. (4.9) returns $a=-I_{i}^{\mathrm{rec}}(t), b=-\left(I_{i}^{\mathrm{c}}-I_{i}^{\mathrm{rec}}(t)-V_{i}(t)\right)$ and $c=I_{i}^{\mathrm{c}}-V_{T}$ with the standard solution obtained from completing the square:

$$
T_{ \pm}=\frac{-b \pm \sqrt{b^{2}-4 a c}}{2 a}
$$

The possible subtraction of equally large numbers in the numerator of Eq. 4.10) incorporates a loss of significance. This should be avoided in the computer simulations using the alternative robust solutions $T_{1}=\frac{d}{a}$ and $T_{2}=\frac{c}{d}$ with $d=-\frac{1}{2}\left(b+\operatorname{sgn}(b) \sqrt{b^{2}-4 a c}\right)$ [97].

The time to the next spike is then $\triangle t_{i}=-\ln T_{i}$ and the smallest next spike time of all neurons defines the next spike time in the network

$$
t_{s+1}=t_{s}+\min _{i}\left\{\triangle t_{i}\right\}
$$

This equation for the next spike time in the network, and the iterative map (2.3) defined with Eq. (4.5)-(4.8) can be used in true event-based simulations. The derivative of the iterative map furthermore yields the single spike Jacobian necessary to apply the approach for the calculation of the Lyapunov spectra.

\subsection{Single Spike Jacobian}

The derivative of the iterative map, the single spike Jacobian is derived similar to Section 2.3. The difference is that each neuron is described by a two dimensional state $\vec{x}_{i}(t)=\left(V_{i}(t), I_{i}(t)\right)$ in the cLIF model. Therefore, the single spike Jacobian will be a $2 N \times 2 N$ matrix. While the explicit expression for the next spike time in the network was necessary for the event-based simulations, this restriction does not occur in the derivation of the single spike Jacobian. The implicit definition of the next spike time is sufficient and the single spike Jacobian is derived for arbitrary ratios $\gamma=\tau_{\mathrm{m}} / \tau_{\mathrm{I}}$

The single spike Jacobian has the following form in cLIF networks:

$$
\mathbf{D}\left(t_{s}\right)=\left(\begin{array}{c|c}
\frac{\mathrm{d} \vec{V}\left(t_{s+1}\right)}{\mathrm{d} \vec{V}\left(t_{s}\right)} & \frac{\mathrm{d} \vec{V}\left(t_{s+1}\right)}{\mathrm{d} \vec{I}\left(t_{s}\right)} \\
\hline \frac{\mathrm{d} \vec{I}\left(t_{s+1}\right)}{\mathrm{d} \vec{V}\left(t_{s}\right)} & \frac{\mathrm{d} \vec{I}\left(t_{s+1}\right)}{\mathrm{d} \vec{I}\left(t_{s}\right)}
\end{array}\right) .
$$

In the derivation of the single spike Jacobian, the iterative map will be shifted by a small amount $\delta$ as discussed in Section 2.3. Then the possibly perturbed spike time of neuron $j^{*}$, denoted $\tau_{s+1}$, lies in the considered interval $\left(t_{s} \rightarrow t_{s}+\delta, t_{s+1} \rightarrow t_{s+1}+\delta\right]$. Whenever appropriate in the derivation of the single spike Jacobian, the final limit $\delta \rightarrow 0$ will be taken and $\tau_{s+1}$ identified with $t_{s+1}$. 
The shifted map reads

$$
x_{i}\left(t_{s+1}\right)= \begin{cases}f\left(x_{i}\left(t_{s}\right), t_{s+1}-t_{s}\right) & \text { if } i \notin \operatorname{post}\left(j^{*}\right) \\ f\left(g\left(f\left(x_{i}\left(t_{s}\right), \tau_{s+1}-t_{s}\right)\right), t_{s+1}-\tau_{s+1}\right) & \text { if } i \in \operatorname{post}\left(j^{*}\right) .\end{cases}
$$

For clarity, the important Eq. (4.5)-(4.8) defining the shifted map (4.13) are rewritten using the abbreviations $T_{\delta}=\exp \left(-\left(t_{s+1}-\tau_{s+1}\right) / \tau_{\mathrm{m}}\right)$ with $\partial T_{\delta} / \partial \tau_{s+1}=1 / \tau_{\mathrm{m}}$ and $T_{s}=\exp \left(-\left(\tau_{s+1}-t_{s}\right) / \tau_{\mathrm{m}}\right)$ with $\partial T_{s} / \partial \tau_{s+1}=-1 / \tau_{\mathrm{m}}$.

The evolution of the neurons' voltages follows:

$$
\begin{aligned}
f\left(V_{i}\left(\tau_{s+1}^{+}\right), T_{\delta}\right) & \equiv V_{i}\left(t_{s+1}\right)=I_{i}^{\mathrm{c}}+\frac{I_{i}^{\mathrm{rec}}\left(\tau_{s+1}^{+}\right)}{1-\gamma} T_{\delta}^{\gamma}-\left(I_{i}^{\mathrm{c}}+\frac{I_{i}^{\mathrm{rec}}\left(\tau_{s+1}^{+}\right)}{1-\gamma}-V_{i}\left(\tau_{s+1}^{+}\right)\right) T_{\delta} \\
g\left(V_{i}\left(\tau_{s+1}^{-}\right)\right) & \equiv V_{i}\left(\tau_{s+1}^{+}\right)=V_{i}\left(\tau_{s+1}^{-}\right) \\
f\left(V_{i}\left(t_{s}\right), T_{s}\right) & \equiv V_{i}\left(\tau_{s+1}^{-}\right)=I_{i}^{\mathrm{c}}+\frac{I_{i}^{\mathrm{rec}}\left(t_{s}\right)}{1-\gamma} T_{s}^{\gamma}-\left(I_{i}^{\mathrm{c}}+\frac{I_{i}^{\mathrm{rec}}\left(t_{s}\right)}{1-\gamma}-V_{i}\left(t_{s}\right)\right) T_{s},
\end{aligned}
$$

The evolution of the recurrent currents follows:

$$
\begin{aligned}
f\left(I_{i}^{\mathrm{rec}}\left(\tau_{s+1}^{+}\right), T_{\delta}\right) & \equiv I_{i}^{\mathrm{rec}}\left(t_{s+1}\right)=I_{i}^{\mathrm{rec}}\left(\tau_{s+1}^{+}\right) T_{\delta}^{\gamma} \\
g\left(I_{i}^{\mathrm{rec}}\left(\tau_{s+1}^{-}\right)\right) & \equiv I_{i}^{\mathrm{rec}}\left(\tau_{s+1}^{+}\right)=I_{i}^{\mathrm{rec}}\left(\tau_{s+1}^{-}\right)+\frac{\gamma J_{i^{*} j^{*}}}{\sqrt{K}} \delta i i^{*} \\
f\left(I_{i}^{\mathrm{rec}}\left(t_{s}\right), \tau_{s+1}-t_{s}\right) & \equiv I_{i}^{\mathrm{rec}}\left(\tau_{s+1}^{-}\right)=I_{i}^{\mathrm{rec}}\left(t_{s}\right) T_{s}^{\gamma} .
\end{aligned}
$$

The velocities of the neurons in terms of the membrane time constant just before and after the spike events, respectively, will be important below. They are

$$
\begin{aligned}
v_{i, s+1}^{-} & =I_{i}^{\mathrm{c}}+I_{i}^{\mathrm{rec}}\left(t_{s+1}^{-}\right)-V_{i}\left(t_{s+1}^{-}\right) \\
v_{i, s+1}^{+} & =I_{i}^{\mathrm{c}}+I_{i}^{\mathrm{rec}}\left(t_{s+1}^{+}\right)-V_{i}\left(t_{s+1}^{+}\right) \\
v_{i, s+1}^{+}-v_{i, s+1}^{-} & =\delta_{i j^{*}}+\frac{\gamma J_{i^{*} j^{*}}}{\sqrt{K}} \delta_{i i^{*}} .
\end{aligned}
$$

The first term in the last equation results from the reset of the spiking neuron $j^{*}$ from $V_{j^{*}}\left(t_{s+1}^{-}\right)=$ $V_{T} \equiv 1$ to $V_{j^{*}}\left(t_{s+1}^{+}\right)=V_{R} \equiv 0$, thus the $\delta_{i j^{*}}$. The second term in Eq. 4.16) results from the update of the synaptic currents of the postsynaptic neurons $i^{*} \in \operatorname{post}\left(j^{*}\right)$ at the spike reception $I_{i^{*}}^{\text {rec }}\left(t_{s+1}^{+}\right)-$ $I_{i^{*}}^{\text {rec }}\left(t_{s+1}^{-}\right)=\gamma J_{i^{*} j^{*}} / \sqrt{K}$. 


\subsubsection{The Voltage-Voltage Block}

We start with the derivation of the upper left block in the single spike Jacobian (4.12) similar to the derivation in Section 2.3. The following partial derivatives will be used in the derivation:

$$
\begin{aligned}
\frac{\partial f\left(V_{i}\left(t_{s+1}\right), T_{\delta}\right)}{\partial V_{k}\left(t_{s+1}\right)} & =T_{\delta} \delta_{i k} \rightarrow \delta_{i k} \\
\frac{\partial f\left(V_{i}\left(\tau_{s+1}^{+}\right), T_{\delta}\right)}{\partial I_{k}^{\mathrm{rec}}\left(\tau_{s+1}^{+}\right)} & =\frac{T_{\delta}^{\gamma}-T_{\delta}}{1-\gamma} \delta_{i k} \rightarrow 0 \\
\frac{\partial f\left(V_{i}\left(\tau_{s+1}^{+}\right), T_{\delta}\right)}{\partial \tau_{s+1}} & =\frac{I_{i}^{\mathrm{rec}}\left(\tau_{s+1}^{+}\right)}{1-\gamma} \frac{\gamma T_{\delta}^{\gamma}}{\tau_{\mathrm{m}}}-\left(I_{i}^{\mathrm{c}}+\frac{I_{i}^{\mathrm{rec}}\left(\tau_{s+1}^{+}\right)}{1-\gamma}-V_{i}\left(\tau_{s+1}^{+}\right)\right) \frac{T_{\delta}}{\tau_{\mathrm{m}}} \\
& \rightarrow-\left(V_{i}\left(t_{s+1}^{+}\right)-I_{i}^{\mathrm{c}}-\frac{I_{i}^{\mathrm{rec}}\left(t_{s+1}^{+}\right)}{1-\gamma}(1-\gamma)\right) \frac{1}{\tau_{\mathrm{m}}} \\
\frac{\partial g\left(V_{i}\left(\tau_{s+1}^{-}\right)\right)}{\partial V_{k}\left(\tau_{s+1}^{-}\right)} & =-\frac{v_{i, s+1}^{+}}{\tau_{\mathrm{m}}} \\
\frac{\partial f\left(V_{i}\left(t_{s}\right), T_{s}\right)}{\partial V_{j}\left(t_{s}\right)} & =T_{s} \delta_{i j} \\
\frac{\partial f\left(V_{i}\left(t_{s}\right), T_{s}\right)}{\partial \tau_{s+1}} & =-\frac{I_{i}^{\mathrm{rec}}\left(t_{s}\right)}{1-\gamma} \frac{\gamma T_{s}^{\gamma}}{\tau_{\mathrm{m}}}+\left(I_{i}^{\mathrm{c}}+\frac{I_{i}^{\mathrm{rec}}\left(t_{s}\right)}{1-\gamma}-V_{i}\left(t_{s}\right)\right) \frac{T_{s}}{\tau_{\mathrm{m}}} \\
& \rightarrow\left(V_{i}\left(t_{s+1}^{-}\right)-I_{i}^{\mathrm{c}}-\frac{I_{i}^{\mathrm{rec}}\left(t_{s+1}^{-}\right)}{1-\gamma}(1-\gamma)\right) \frac{1}{\tau_{\mathrm{m}}} \\
\tau_{\mathrm{m}}^{-} &
\end{aligned}
$$

Furthermore, we will need derivative $\frac{\mathrm{d} \tau_{s+1}}{\mathrm{~d} V_{j}\left(t_{s}\right)}$, which is obtained from the implicit definition of the next spike time (4.9), solved for the voltage of the spiking neuron:

$$
V_{j^{*}}\left(t_{s}\right)=\frac{1-I_{j^{*}}^{\mathrm{c}}}{T_{S}}-\frac{I_{j^{*}}^{\mathrm{rec}}\left(t_{s}\right)}{1-\gamma} T_{s}^{\gamma-1}+I_{j^{*}}^{\mathrm{c}}+I_{j^{*}}^{\mathrm{rec}}(t) .
$$

The necessary derivative $\frac{\mathrm{d} \tau_{s+1}}{\mathrm{~d} V_{j}\left(t_{s}\right)}$ is calculated as follows:

$$
\begin{aligned}
\frac{\mathrm{d} V_{j^{*}}\left(t_{s}\right)}{\mathrm{d} \tau_{s+1}} & =-\frac{1-I_{j^{*}}^{\mathrm{c}}-T_{s}}{T_{s}^{2}} \frac{I_{j^{*}}^{\mathrm{rec}}\left(t_{s}\right)}{1-\gamma} \frac{\gamma-1}{-\tau_{\mathrm{m}}} T_{s}^{\gamma-1} \\
& =-\frac{I_{j^{*}}^{\mathrm{c}}+I_{j^{*}}^{\mathrm{rec}}\left(t_{s}\right) T_{s}^{\gamma}-1}{T_{s} \tau_{\mathrm{m}}} \\
& =-\frac{v_{j^{*}, s+1}}{T_{s} \tau_{\mathrm{m}}}
\end{aligned}
$$




$$
\frac{\mathrm{d} \tau_{s+1}}{\mathrm{~d} V_{j^{*}}\left(t_{s}\right)}=-\frac{T_{s} \tau_{\mathrm{m}}}{v_{j^{*}, s+1}^{-}}
$$

With these prerequisites, the explicit derivation of the upper right block in (4.12) goes:

$$
\begin{aligned}
& \frac{\mathrm{d} V_{i}\left(t_{s+1}\right)}{\mathrm{d} V_{j}\left(t_{s}\right)}=\underbrace{\frac{\partial V_{i}\left(t_{s+1}\right)}{\partial V_{k}\left(\tau_{s+1}^{+}\right)}}_{\stackrel{4.17}{\longrightarrow} \delta_{i k}} \frac{\mathrm{d} V_{k}\left(\tau_{s+1}^{+}\right)}{\mathrm{d} V_{j}\left(t_{s}\right)}+\underbrace{\stackrel{\partial V_{i}\left(t_{s+1}\right)}{\frac{\mathrm{rec}\left(\tau_{s+1}^{+}\right)}{\longrightarrow} 0}}_{[4.18} \frac{\mathrm{d} I_{k}^{\mathrm{rec}}\left(\tau_{s+1}^{+}\right)}{\mathrm{d} V_{j}\left(t_{s}\right)}+\underbrace{\frac{\partial V_{i}\left(t_{s+1}\right)}{\partial \tau_{s+1}}}_{\stackrel{4.19}{\longrightarrow}-\frac{v_{i, s+1}^{+}}{\tau_{\mathrm{m}}}} \frac{\mathrm{d} \tau_{s+1}}{\mathrm{~d} V_{j}\left(t_{s}\right)}
\end{aligned}
$$

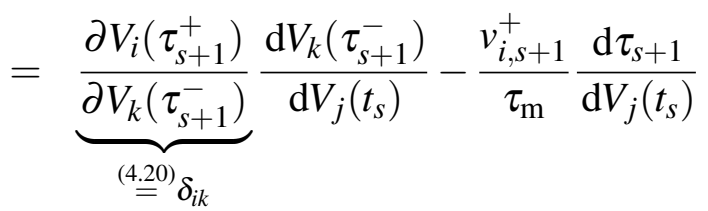

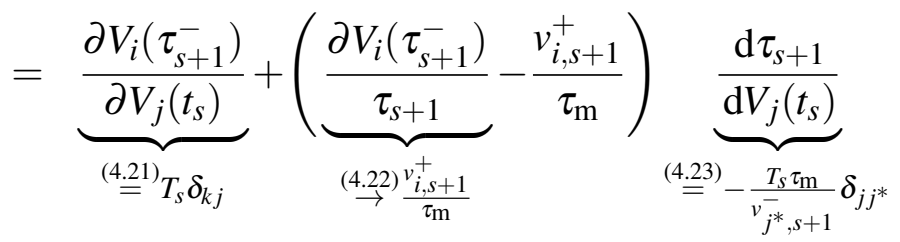

$$
\begin{aligned}
& \frac{\mathrm{d} V_{i}\left(t_{s+1}\right)}{\mathrm{d} V_{j}\left(t_{s}\right)}=T_{s}\left(1+\frac{1}{v_{j^{*}, s+1}^{-}} \delta_{i j^{*}} \delta_{j j^{*}}+\frac{\gamma J_{i^{*} j^{*}}}{v_{j^{*}, s+1}^{-} \sqrt{K}} \delta_{i i^{*}} \delta_{j j^{*}}\right),
\end{aligned}
$$

where Eq. 4.16) was used in the last step. The first two terms in the bracket constitute the diagonal elements and the third term the offdiagonal elements of the spike receiving neurons. Note that the diagonal element of the spiking neuron can also be written as $T_{s} v_{j^{*}, s+1}^{+} / v_{j^{*}, s+1}^{-}$(Eq. (4.16). The other parts of the single spike Jacobian (4.12) are derived accordingly.

\subsubsection{The Current-Voltage Block}

For the lower left block in the single spike Jacobian 4.12), the following additional partial derivatives are used:

$$
\begin{aligned}
\frac{\partial f\left(I_{i}^{\mathrm{rec}}\left(t_{s+1}\right), T_{\delta}\right)}{\partial I_{k}^{\mathrm{rec}}\left(\tau_{s+1}^{+}\right)} & =T_{\delta}^{\gamma} \delta_{i k} \rightarrow \delta_{i k} \\
\frac{\partial f\left(I_{i}^{\mathrm{rec}}\left(\tau_{s+1}^{+}\right), T_{\delta}\right)}{\partial \tau_{s+1}} & =\frac{\gamma}{\tau_{\mathrm{m}}} I_{i}^{\mathrm{rec}}\left(\tau_{s+1}^{+}\right) T_{\delta}^{\gamma} \rightarrow \frac{\gamma}{\tau_{\mathrm{m}}} I_{i}^{\mathrm{rec}}\left(\tau_{s+1}^{+}\right) \\
\frac{\partial f\left(I_{i}^{\mathrm{rec}}\left(t_{s}\right), T_{s}\right)}{\partial \tau_{s+1}} & =-\frac{\gamma}{\tau_{\mathrm{m}}} I_{i}^{\mathrm{rec}}\left(t_{s}\right) T_{s}^{\gamma}=-\frac{\gamma}{\tau_{\mathrm{m}}} I_{i}^{\mathrm{rec}}\left(\tau_{s+1}^{-}\right) \\
I_{i}^{\mathrm{rec}}\left(t_{s+1}^{+}\right)-I_{i}^{\mathrm{rec}}\left(t_{s+1}^{-}\right) & =\frac{\gamma J_{i^{*} j^{*}}}{\sqrt{K}} \delta_{i i^{*}} .
\end{aligned}
$$

The last equation results from update of the synaptic currents of the postsynaptic neurons $i^{*} \in$ $\operatorname{post}\left(j^{*}\right)$ at the spike reception. With these additional partial derivatives, the lower left part of the single spike Jacobian (4.12) can be derived as follows: 


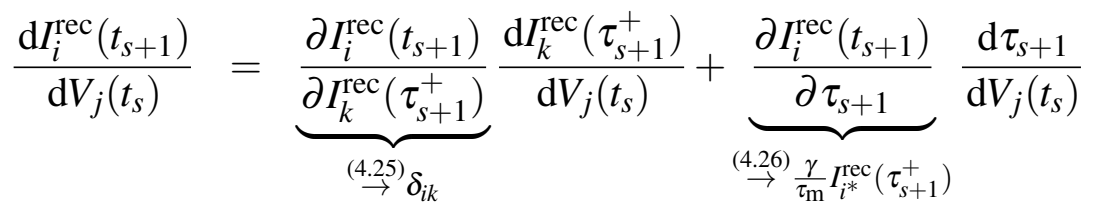

$$
\begin{aligned}
& =\underbrace{\frac{\partial I_{i}^{\mathrm{rec}}\left(\tau_{s+1}^{+}\right)}{\partial I_{k}^{\mathrm{rec}}\left(\tau_{s+1}^{-}\right)}}_{=\delta_{i k}} \frac{\mathrm{d} I_{k}^{\mathrm{rec}}\left(\tau_{s+1}^{-}\right)}{\mathrm{d} V_{j}\left(t_{s}\right)}+\frac{\gamma}{\tau_{\mathrm{m}}} I_{i}^{\mathrm{rec}}\left(\tau_{s+1}^{+}\right) \frac{\mathrm{d} \tau_{s+1}}{\mathrm{~d} V_{j}\left(t_{s}\right)}
\end{aligned}
$$

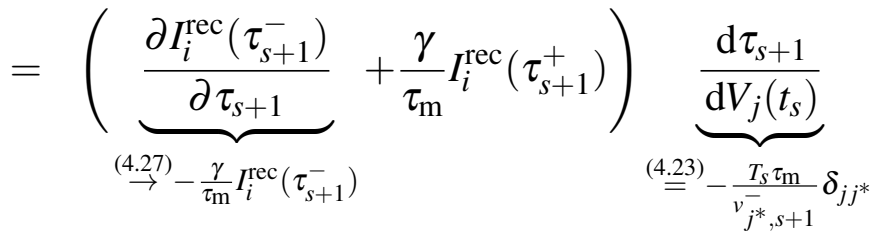

$$
\begin{aligned}
& \frac{\mathrm{d} I_{i}^{\mathrm{rec}}\left(t_{s+1}\right)}{\mathrm{d} V_{j}\left(t_{s}\right)}=-\gamma T_{s} \frac{\gamma J_{i^{*} j^{*}}}{v_{j^{*}, s+1}^{-} \sqrt{K}} \delta_{i i^{*}} \delta_{j j^{*}},
\end{aligned}
$$

where Eq. 4.28) was used in the last step. The lower left part of the Jacobian 4.12) thus consists of just the nondiagonal elements of the spike receiving neurons. We will continue counterclockwise with the derivation of the lower right part of the Jacobian.

\subsubsection{The Current-Current Block}

To derive the lower right block of the single spike Jacobian (4.12), it is necessary to calculate the derivative $\frac{\mathrm{d} \tau_{s+1}}{\mathrm{~d} I_{j^{*}}{ }^{\text {ec }}\left(t_{s}\right)}$, which is obtained from the implicitly defined next spike time (4.9) solved for the recurrent current of the spiking neuron:

$$
I_{j^{*}}^{\mathrm{rec}}\left(t_{s}\right)=\left(I_{j^{*}}^{\mathrm{c}}-V_{j^{*}}\left(t_{s}\right)\right) T_{s}-\left(I_{j^{*}}^{\mathrm{c}}-1\right) \frac{1-\gamma}{T_{S}^{\gamma}-T_{S}} .
$$

The necessary derivative $\frac{\partial \tau_{s+1}}{\partial l_{j^{*}}^{\mathrm{rec}}\left(t_{s}\right)}$ is calculated as follows:

$$
\begin{aligned}
& \frac{\mathrm{d} I_{j^{*}}^{\mathrm{rec}}\left(t_{s}\right)}{\mathrm{d} \tau_{s+1}}=\frac{\left(I_{j^{*}}^{\mathrm{c}}-V_{j^{*}}\left(t_{s}\right)\right)\left(T_{s}^{\gamma}-T_{s}\right)(1-\gamma)-I_{j^{*}}^{\mathrm{rec}}\left(t_{s}\right)\left(T_{s}^{\gamma}-T_{s}\right)\left(\gamma T_{s}^{\gamma-1}-1\right)}{\left(T_{s}^{\gamma}-T_{s}\right)^{2}} \frac{T_{s}}{-\tau_{\mathrm{m}}} \\
&=\left(-\left(I_{j^{*}}^{\mathrm{c}}+\frac{I_{j^{*}}^{\mathrm{rec}}\left(t_{s}\right)}{1-\gamma}-V_{j^{*}}\left(t_{s}\right)\right) \frac{T_{s}}{\tau_{\mathrm{m}}}+\frac{I_{j^{*}}^{\mathrm{rec}}\left(t_{s}\right)}{1-\gamma} \frac{\gamma T_{s}^{\gamma}}{\tau_{\mathrm{m}}}\right) \frac{1-\gamma}{T_{s}^{\gamma}-T_{s}} \\
& \stackrel{4.22 \gamma}{=}-\frac{v_{j^{*}, s+1}^{-}}{\tau_{\mathrm{m}}} \frac{1-\gamma}{T_{s}^{\gamma}-T_{s}} \\
& \frac{\mathrm{d} \tau_{s+1}}{\mathrm{~d} I_{j^{*}}^{\mathrm{rec}}\left(t_{s}\right)}=-\frac{\tau_{\mathrm{m}}}{v_{j^{*}, s+1}^{\gamma}} \frac{T_{s}^{\gamma}-T_{s}}{1-\gamma},
\end{aligned}
$$


We also need one more partial derivative:

$$
\frac{\partial f\left(I_{i}^{\mathrm{rec}}\left(t_{s}\right), T_{s}\right)}{\partial I_{j}^{\mathrm{rec}}\left(t_{s}\right)}=T_{s}^{\gamma} \delta_{l j}
$$

With these prerequisites, the lower right part of the single spike Jacobian (4.12) is derived as follows:

$$
\begin{aligned}
& \frac{\mathrm{d} I_{i}^{\mathrm{rec}}\left(t_{s+1}\right)}{\mathrm{d} I_{j}^{\mathrm{rec}}\left(t_{s}\right)}=\underbrace{\frac{\partial I_{i}^{\mathrm{rec}}\left(t_{s+1}\right)}{\partial I_{k}^{\mathrm{rec}}\left(\tau_{s+1}^{+}\right)}}_{\stackrel{4.25}{\longrightarrow} \delta_{i k}} \frac{\mathrm{d} I_{k}^{\mathrm{rec}}\left(\tau_{s+1}^{+}\right)}{\mathrm{d} I_{j}^{\mathrm{rec}}\left(t_{s}\right)}+\underbrace{\frac{\partial I_{i}^{\mathrm{rec}}\left(t_{s+1}\right)}{\partial \tau_{s+1}}}_{\stackrel{4.26}{\longrightarrow} \frac{\gamma}{\tau_{\mathrm{m}}} I_{i^{*}}^{\mathrm{rec}}\left(\tau_{s+1}^{+}\right)} \frac{\mathrm{d} \tau_{s+1}}{\mathrm{~d} I_{j}^{\mathrm{rec}}\left(t_{s}\right)} \\
& =\underbrace{\frac{\partial I_{i}^{\mathrm{rec}}\left(\tau_{s+1}^{+}\right)}{\partial I_{k}^{\mathrm{rec}}\left(\tau_{s+1}^{-}\right)}}_{=\delta_{i k}} \frac{\mathrm{d} I_{k}^{\mathrm{rec}}\left(\tau_{s+1}^{-}\right)}{\mathrm{d} I_{j}^{\mathrm{rec}}\left(t_{s}\right)}+\frac{\gamma}{\tau_{\mathrm{m}}} I_{i}^{\mathrm{rec}}\left(\tau_{s+1}^{+}\right) \frac{\mathrm{d} \tau_{s+1}}{\mathrm{~d} I_{j}^{\mathrm{rec}}\left(t_{s}\right)} \\
& =\underbrace{\frac{\partial I_{i}^{\mathrm{rec}}\left(\tau_{s+1}^{-}\right)}{\partial I_{j}^{\mathrm{rec}}\left(t_{s}\right)}}_{\stackrel{4.31}{=} T_{s}^{\gamma} \delta_{l j}}+(\underbrace{\frac{\partial I_{i}^{\mathrm{rec}}\left(\tau_{s+1}^{-}\right)}{\partial \tau_{s+1}}}_{\stackrel{4.27}{\longrightarrow}-\frac{\gamma}{\tau_{\mathrm{m}}} I_{i}^{\mathrm{rec}}\left(\tau_{s+1}^{-}\right)}+\frac{\gamma}{\tau_{\mathrm{m}}} I_{i}^{\mathrm{rec}}\left(\tau_{s+1}^{+}\right)) \underbrace{\frac{\mathrm{d} \tau_{s+1}}{\mathrm{~d} r_{j}^{\mathrm{rec}}\left(t_{s}\right)}}_{\frac{4.28}{-}-\frac{\tau_{\mathrm{m}}}{v_{j^{*}, s+1}^{-}} \frac{T_{s}^{\gamma}-T_{s}}{1-\gamma} \delta_{j j^{*}}} \\
& \frac{\mathrm{d} I_{i}^{\mathrm{rec}}\left(t_{s+1}\right)}{\mathrm{d} I_{j}^{\mathrm{rec}}\left(t_{s}\right)}=T_{s}^{\gamma} \delta_{i j}-\frac{\gamma\left(T_{s}^{\gamma}-T_{s}\right)}{1-\gamma} \frac{\gamma J_{i^{*} j^{*}}}{v_{j^{*}, s+1}^{-} \sqrt{K}} \delta_{i i^{*}} \delta_{j j^{*}},
\end{aligned}
$$

where Eq. 4.28) was used in the last step.

\subsubsection{The Voltage-Current Block}

The remaining upper right block of the single spike Jacobian (4.12) is derived analogously to the previous blocks, with one additional partial derivative:

$$
\frac{\partial f\left(V_{i}\left(t_{s}\right), T_{s}\right)}{\partial I_{j}^{\mathrm{rec}}\left(t_{s}\right)}=\frac{T_{s}^{\gamma}-T_{s}}{1-\gamma} \delta_{i j}
$$

The upper right block of the single spike Jacobian is:

$$
\begin{aligned}
& \frac{\mathrm{d} V_{i}\left(t_{s+1}\right)}{\mathrm{d} I_{j}^{\mathrm{rec}}\left(t_{s}\right)}=\underbrace{\frac{\partial V_{i}\left(t_{s+1}\right)}{\partial V_{k}\left(\tau_{s+1}^{+}\right)}}_{\stackrel{4.17}{\longrightarrow} \delta_{i k}} \frac{\mathrm{d} V_{k}\left(\tau_{s+1}^{+}\right)}{\mathrm{d} I_{j}^{\mathrm{rec}}\left(t_{s}\right)}+\underbrace{\frac{\partial V_{i}\left(t_{s+1}\right)}{\partial I_{i}^{\mathrm{rec}}\left(\tau_{s+1}^{+}\right)}}_{\stackrel{4.18}{\longrightarrow} 0} \frac{\mathrm{d} I_{i}^{\mathrm{rec}}\left(\tau_{s+1}^{+}\right)}{\mathrm{d} I_{j}^{\mathrm{rec}}\left(t_{s}\right)}+\underbrace{\frac{\partial V_{i}\left(t_{s+1}\right)}{\partial \tau_{s+1}}}_{\stackrel{4.19}{\longrightarrow}-v_{i, s+1}^{+} / \tau_{\mathrm{m}}} \frac{\mathrm{d} \tau_{s+1}}{\mathrm{~d} I_{j}^{\mathrm{rec}}\left(t_{s}\right)} \\
& =\underbrace{\frac{\partial V_{i}\left(\tau_{s+1}^{+}\right)}{\partial V_{k}\left(\tau_{s+1}^{-}\right)}}_{\frac{4.20}{-} \delta_{i k}} \frac{\mathrm{d} V_{k}\left(\tau_{s+1}^{-}\right)}{\mathrm{d} I_{j}^{\mathrm{rec}}\left(t_{s}\right)}-\frac{v_{i, s+1}^{+}}{\tau_{\mathrm{m}}} \frac{\mathrm{d} \tau_{s+1}}{\mathrm{~d} I_{j}^{\mathrm{rec}}\left(t_{s}\right)}
\end{aligned}
$$




$$
\begin{aligned}
& =\underbrace{\frac{\partial V_{i}\left(\tau_{s+1}^{-}\right)}{\partial I_{j}^{\mathrm{rec}}\left(t_{s}\right)}}_{\stackrel{4.33)}{-\frac{T_{s}^{\gamma}-T_{s}}{1-\gamma} \delta_{i j}}}+(\underbrace{\frac{\partial V_{i}\left(\tau_{s+1}^{-}\right)}{\tau_{s+1}}}_{\stackrel{4.222}{\longrightarrow} v_{i, s+1}^{-} / \tau_{\mathrm{m}}}-\frac{v_{i, s+1}^{+}}{\tau_{\mathrm{m}}}) \underbrace{\frac{\mathrm{d} \tau_{s+1}}{\mathrm{~d} I_{j}^{\mathrm{rec}}\left(t_{s}\right)}}_{\frac{4.28}{-}-\frac{\left(T_{s}^{\gamma}-T_{s}\right) /(1-\gamma)}{v_{j^{*}, s+1}^{-} / \tau_{\mathrm{m}}}} \delta_{j j^{*}} \\
& \frac{\mathrm{d} V_{i}\left(t_{s+1}\right)}{\mathrm{d} I_{j}^{\mathrm{rec}}\left(t_{s}\right)}=\frac{\left(T_{s}^{\gamma}-T_{s}\right)}{1-\gamma}\left(\delta_{i j}+\frac{1}{v_{j^{*}, s+1}^{-}} \delta_{i j^{*}} \delta_{j j^{*}}+\frac{\gamma J_{i^{*} j^{*}}}{v_{j^{*}, s+1}^{-} \sqrt{K}}\right) \delta_{i i^{*}} \delta_{j j^{*}},
\end{aligned}
$$

where Eq. (4.28) and (4.28) were used in the last step.

Finally, Eq.4.24), 4.29), 4.34) and (4.34) can be combined to the full single spike Jacobian of the correlated leaky integrate and fire model:

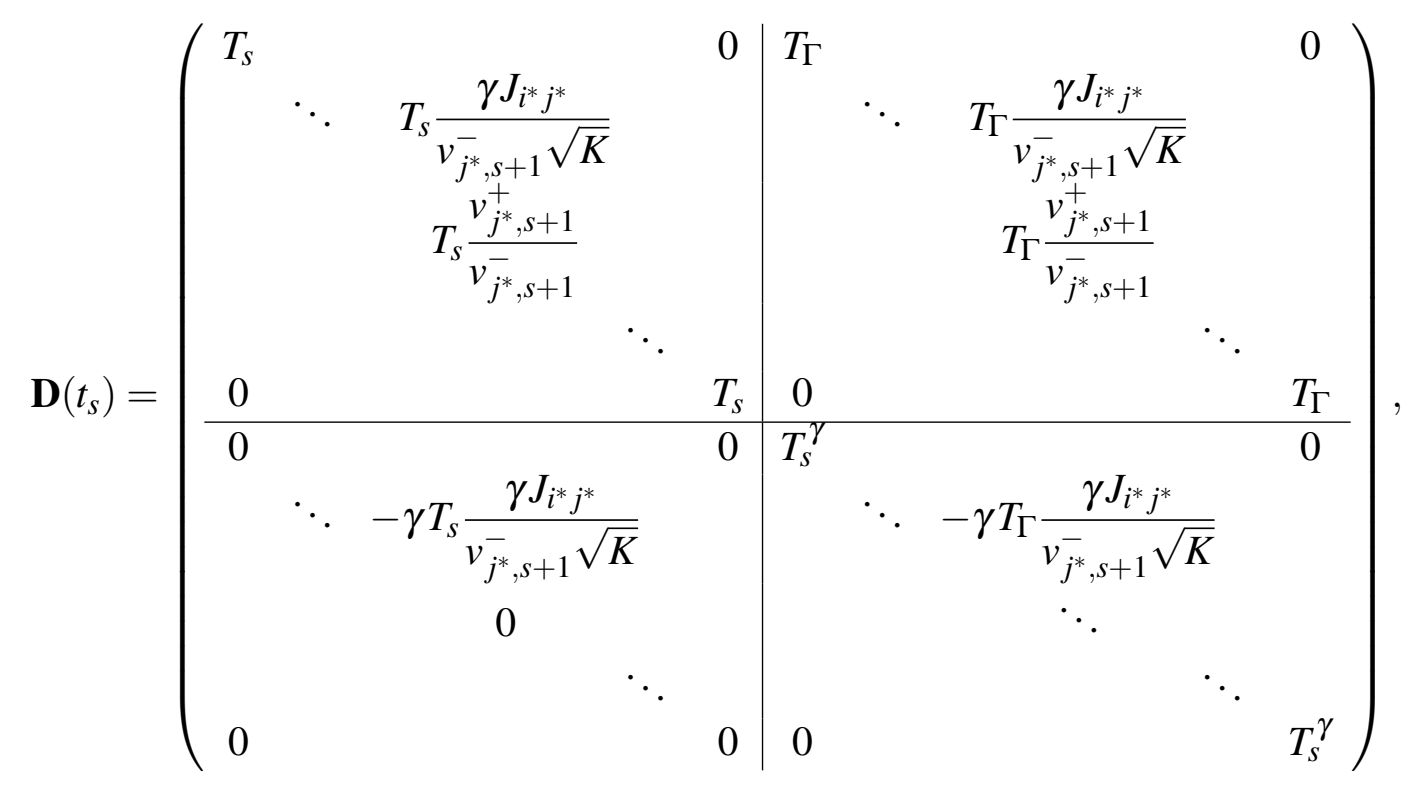

where the terms $T_{\Gamma}=\left(T_{s}^{\gamma}-T_{s}\right) /(1-\gamma)$ and $1+1 / v_{j^{*}, s+1}^{-}=v_{j^{*}, s+1}^{+} / v_{j^{*}, s+1}^{-}$are used for a compact presentation. The non-diagonal elements are in the $j^{*}$-th and $N+j^{*}$-th column and $i^{*} \in$ post $j^{*}$ rows.

The derivation of the single spike Jacobian (4.35) for cLIF networks paves the way for the calculation of the complete Lyapunov spectra in the same procedure discussed in Chapter2. The only difference is that there are two dimensions per neuron, and the phase space is therefore $2 \mathrm{~N}$ dimensional. The single spike Jacobian is ordered in a way that in a vector $\vec{g}$ evolved with the single spike Jacobian $\mathbf{D}\left(t_{s}\right)$, the first $N$ elements $g_{1} \ldots g_{N}$ represent a perturbation of the voltages of the neurons and the second $N$ elements $g_{N+1} \ldots g_{2 N}$ represent a perturbation of the currents of the neurons. 


\subsubsection{Mean Lyapunov Exponent}

The mean Lyapunov exponent can be derived in a random matrix approximation, using the single spike Jacobian (4.35) similar to the derivation in Section 2.5.1. With the long term Jacobian $\mathbf{T}=$ $\mathbf{D}\left(t_{s}\right) \cdots \mathbf{D}(0)$, the mean Lyapunov exponent $\bar{\lambda}=\frac{1}{2 N} \sum_{i=1}^{2 N} \lambda_{i}$ is defined as

$$
\begin{aligned}
\bar{\lambda} & =\frac{1}{2 N} \lim _{s \rightarrow \infty} \frac{1}{t_{s}} \ln (\operatorname{det} \mathbf{T}) \\
& =\frac{1}{2 N} \lim _{s \rightarrow \infty} \frac{1}{t_{s}} \sum_{p=1}^{s} \ln \left(\operatorname{det} \mathbf{D}\left(t_{p}\right)\right) .
\end{aligned}
$$

Assuming the single spike Jacobians to be random matrices of the form (4.35) with independent and identically distributed random elements $d\left(I^{*}\right)=v^{+} / v^{-}$, the determinant of the random matrices is $\operatorname{det} \mathbf{D}=T^{N}\left(d\left(I^{*}\right)\right) T^{\gamma N}$ with $T=\exp \left(-\triangle_{t} / \tau_{\mathrm{m}}\right)$. Here and in the following, we also assume an asynchronous state in which the average time between two spikes in the network is $\triangle_{t}=1 /(N \bar{v})$. The random elements $d\left(I^{*}\right)$ depend on the current distribution at the times when neurons emit a spike. From Eq. (4.15) and (4.14) follows for the spiking neuron $j^{*}$ :

$$
d\left(I^{*}\right)=\frac{v^{+}}{v^{-}}=1+\frac{1}{I_{j^{*}}^{\mathrm{c}}+I_{j^{*}}^{\mathrm{rec}}\left(t_{s+1}\right)-1}=1+\frac{1}{I^{*}-1},
$$

where $I^{*}=I_{j^{*}}^{\mathrm{c}}+I_{j^{*}}^{\mathrm{rec}}\left(t_{s+1}\right)$ denotes the current of the spiking neuron at the spike time, with probability distribution $P\left(I^{*}\right)$.

The number of spike events per unit time is $\lim _{s \rightarrow \infty} \frac{1}{t_{s}} \sum_{p=1}^{s} 1=N \bar{v}$. Thus, in the random matrix approximation, the mean Lyapunov exponent for inhibitory networks becomes

$$
\begin{aligned}
\bar{\lambda} & \approx \frac{1}{2 N} N \bar{v} \int \ln \left(T^{N(\gamma+1)} d\left(I^{*}\right)\right) P\left(I^{*}\right) \mathrm{d} I^{*} \\
& =\frac{\bar{v}}{2}\left(\ln \left(T^{N(\gamma+1)}\right)+\int \ln \left(d\left(I^{*}\right)\right) P\left(I^{*}\right) \mathrm{d} I^{*}\right) \\
& =\frac{(\gamma+1) N \bar{v}}{2 N \bar{v} \tau_{\mathrm{m}}}+\frac{\bar{v}}{2} \int \ln \left(1+\frac{1}{I^{*}-1}\right) P\left(I^{*}\right) \mathrm{d} I^{*} \\
& \approx \frac{\gamma+1}{2 \tau_{\mathrm{m}}} \\
& =\frac{1}{2}\left(\frac{1}{\tau_{\mathrm{I}}}+\frac{1}{\tau_{\mathrm{m}}}\right) .
\end{aligned}
$$

In the last approximation we assumed that the neurons' currents $I^{*}$ at times when they emit a spike are considerably above the rheobase current $I_{T}=1$, such that the integral vanishes because of $1 /\left(I^{*}-1\right) \approx 0$. Interestingly, the mean Lyapunov exponent 4.36 is then fully determined by the inverse of the harmonic mean of the membrane time constant $\tau_{\mathrm{m}}$ and the synaptic time constant $\tau_{\mathrm{I}}$. In fact, the random matrix approximation (4.36) agrees excellently with the mean Lyapunov exponent calculated from the numerical simulations (Fig. 4.5 E). 

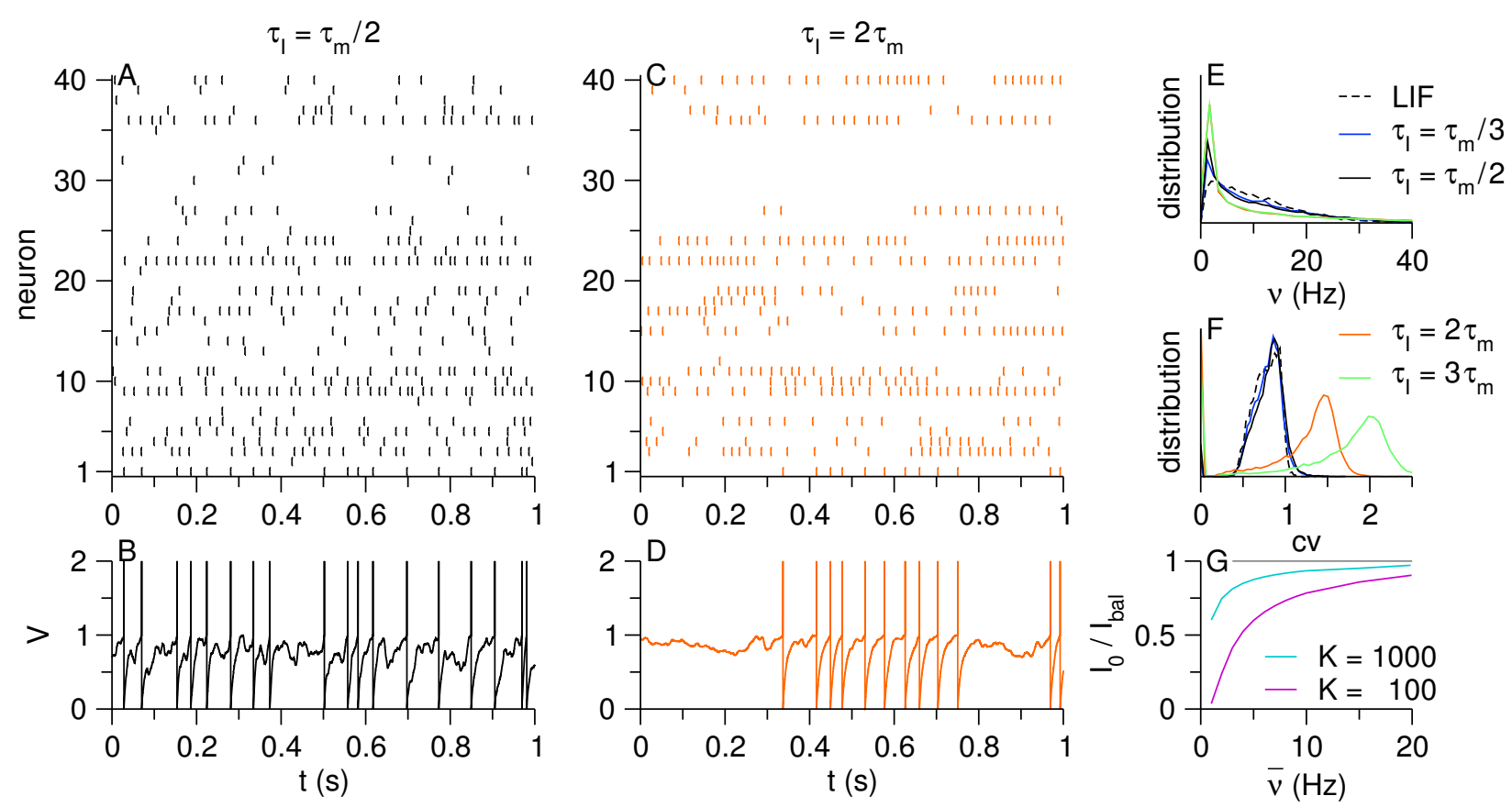

Figure 4.2 - Firing statistics in inhibitory correlated integrate and fire neuron networks with different synaptic time constants $\tau_{\mathrm{I}}$. $(\mathrm{A}, \mathrm{C})$ spike trains of 40 randomly chosen neurons for two synaptic time constants $\tau_{\mathrm{I}}=5 \mathrm{~ms}$ and $\tau_{\mathrm{I}}=20 \mathrm{~ms}$, (B,D) voltage trace of first randomly chosen neuron, (E) firing rate distributions of cLIF networks and LIF networks with $\delta$ pulse coupling (dashed line), (F) coefficients of variation distributions as in $\mathrm{E},(\mathrm{G})$ suprathreshold external current $I_{0}$ in relation to estimated balanced current $I_{\mathrm{bal}}=\bar{v} J_{0} \tau_{\mathrm{m}}$ from balance equation (2.19) versus target average firing rate $\bar{v}$ for synaptic time constants $\tau_{\mathrm{I}}=5 \mathrm{~ms}$ and two different connectivities $K$, (parameters: $N=10000, K=1000, \bar{v}=10 \mathrm{~Hz}, \tau_{\mathrm{m}}=10 \mathrm{~ms}$; all simulation with identical network realizations).

\subsection{Balanced State in Correlated Leaky Integrate and Fire Networks}

The exact expressions for the evolution of the voltage and currents of each neuron in the network, Eq. (4.5)-(4.8) and the next spike time in the network, Eq. (4.11) are used for numerically exact simulations and with the derived single spike Jacobians 4.35), we will calculate the complete Lyapunov spectra in the standard procedure [68,69]. We will study random networks in the balanced state as in Chapter 2 and 3 but now composed of leaky integrate and fire neurons with exponentially decaying postsynaptic currents. Four different synaptic time constants $\tau_{\text {I }}$ relative to the membrane time constant $\tau_{\mathrm{m}}$ will be considered: $\tau_{\mathrm{I}} / \tau_{\mathrm{m}}=\frac{1}{3}, \frac{1}{2}, 2,3$. Because $\tau_{\mathrm{m}}$ can be chosen differently, these ratios should represent different types of synapses with fast or slow GABA receptors (inhibitory), fast AMPA or slow NMDA receptors (both excitatory). Realistic values of synaptic time constants can range from few milliseconds to hundreds of milliseconds. We will mostly focus on $\tau_{\mathrm{m}}=10 \mathrm{~ms}$ and $\tau_{\mathrm{I}}=5 \mathrm{~ms}$, which are realistic values for GABA and AMPA receptors.

An overview of the firing activity in inhibitory cLIF networks is given in Fig. 4.2. It shows two representative spike patterns for cLIF networks with fast and slow synapses (Fig. 4.2 A,C). In both cases the networks exhibit the typical asynchronous irregular activity in the balanced state. A longer synaptic time constant, however, led to a more burst-like firing of the neurons. This is also visible in the represented voltage traces of the same neuron but with different synaptic 

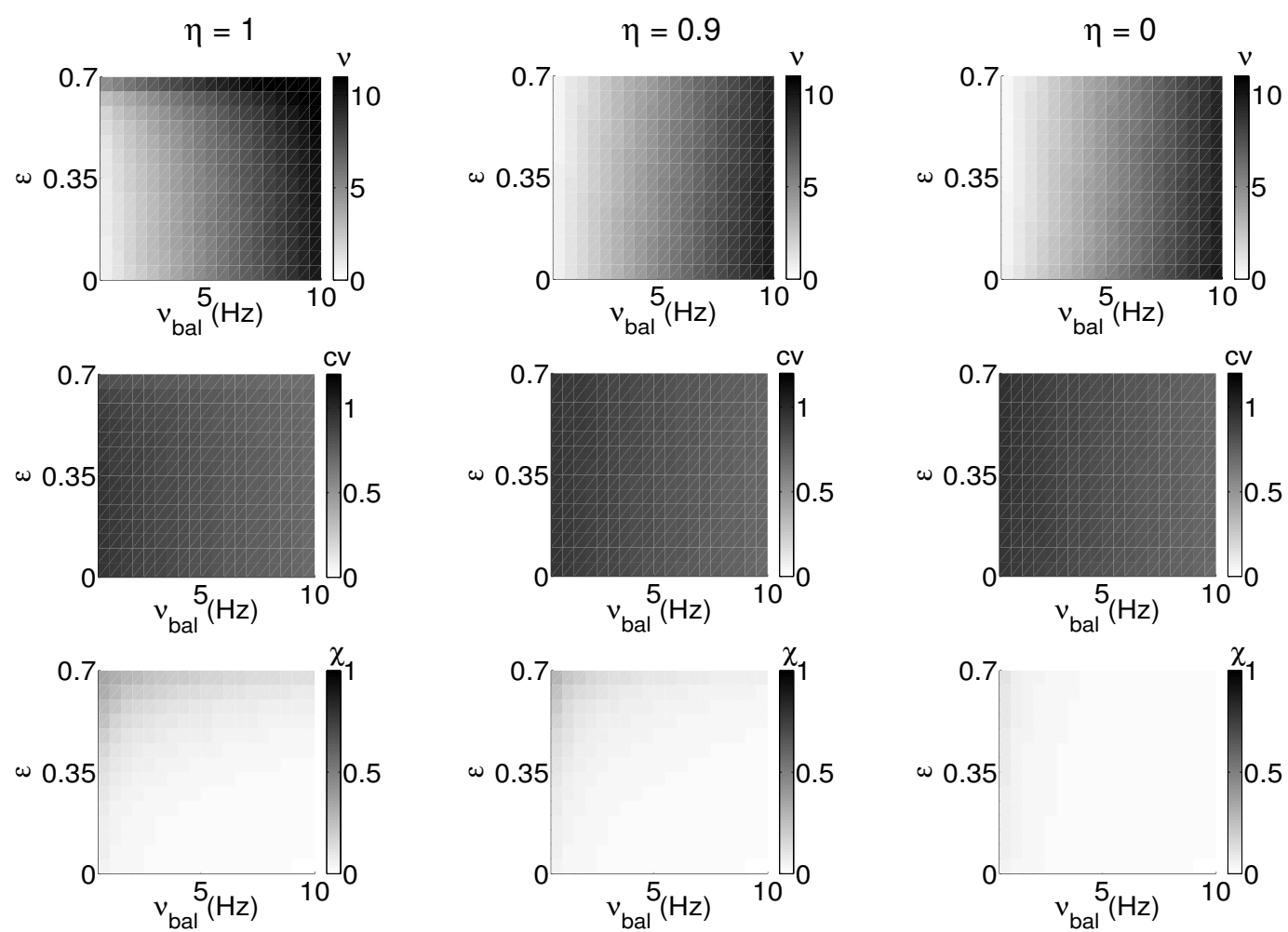

(a) $\tau_{\mathrm{I}}=5 \mathrm{~ms}$
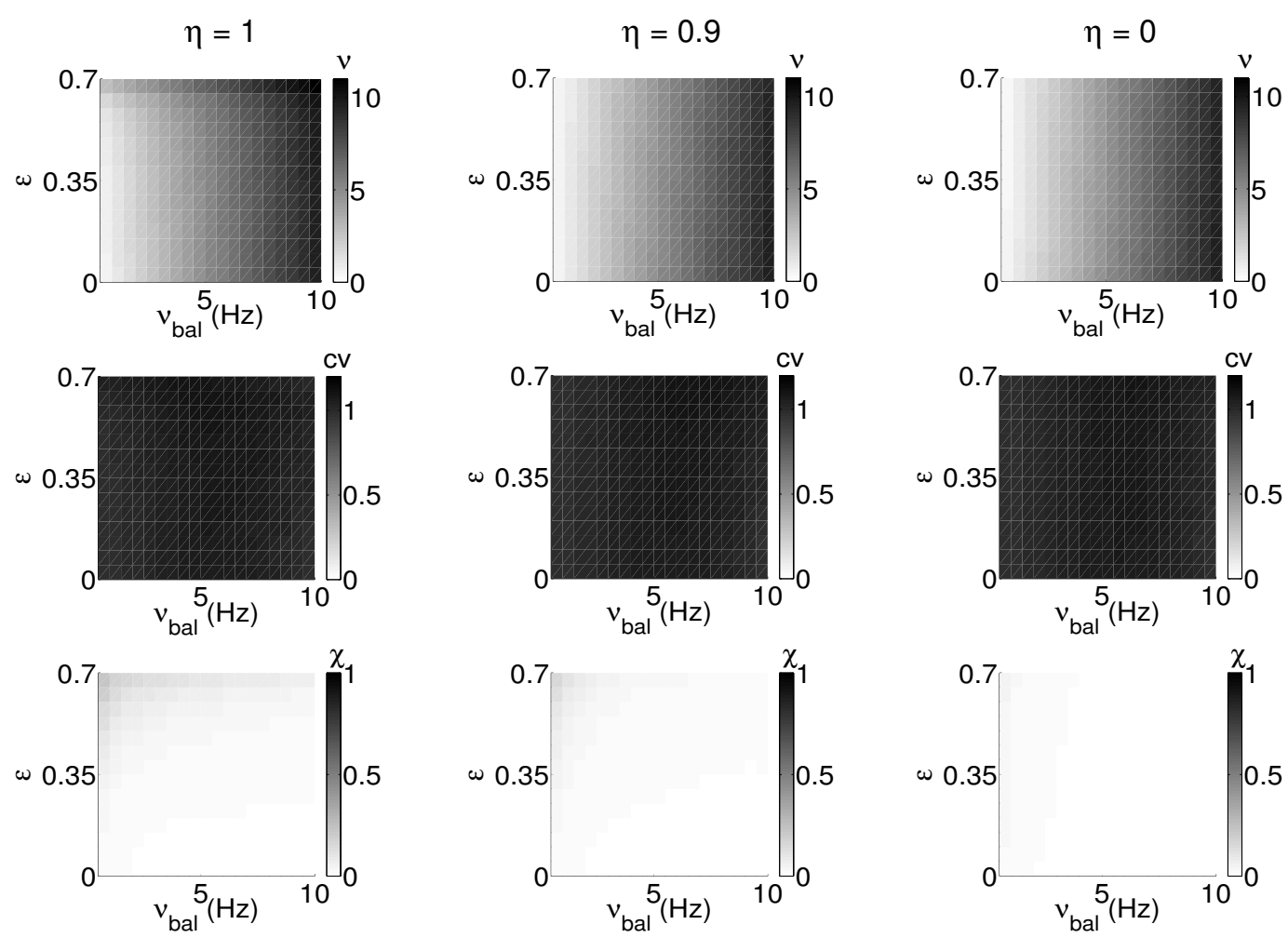

(b) $\tau_{\mathrm{I}}=20 \mathrm{~ms}$

Figure 4.3 - Phase diagrams of excitatory-inhibitory networks for different ratios $\eta=J_{E E} / J_{I E}$. (see coupling matrix (2.31) Columns from left to right: $\eta=1,0.9,0$, from top to bottom: average firing rate $\bar{v}$, coefficient of variation $\mathrm{cv}$ and synchrony measure $\chi$; on the $\mathrm{x}$-axis is plotted the target average firing rate according to the balance equation (2.25) and on the y-axis the excitatory-inhibitory feedback loop activation $\varepsilon$, (parameters: $N_{E}=8000, N_{I}=2000, K=1000, J_{0}=1, \tau_{\mathrm{m}}=10 \mathrm{~ms}$; displayed are averages of 10 runs with different network realizations). 
time constants (Fig. 4.2B,D) and reflected in the shifted distribution of coefficients of variation (Fig. 4.2F). For fast synapses, the distribution was identical with the one in LIF networks with $\delta$ coupling, but slow synapses resulted in average cv values larger than one, which is typical for burst-like firing activity. From the spike pattern, one can infer that there were more neurons that did not spike at all. This is also reflected in the distribution of firing rates in the networks which showed a considerable peak at very low firing rates (Fig. 4.2E). For large synaptic time constants this can even lead to a singularity at zero frequency. This is an interesting phenomenon of a state with mostly silent neurons and referred to as the dark matter in the brain [98, 99].

A difference in the emergence of the balanced state in cLIF networks compared to theta neuron networks is the need for strong recurrent inhibition. In Fig. $4.2 \mathrm{G}$ are plotted the suprathreshold external currents $I_{0}$ relative to the expectation from the balanced equation (2.19) $I_{\mathrm{bal}}=J_{0} \bar{v} \tau_{\mathrm{m}}$ for a given target network-averaged firing rate $\bar{v}$. A ratio of one corresponds to perfect agreement. In cLIF networks with low connectivity or low average firing rate the external currents strongly deviated from the expected value. We suspect that this reflects the logarithmic onset of firing in the FI-curve (Fig. 4.1C). If the recurrent inhibition is weak, the external currents must be extremely low to yield a low networks-average firing rate. Therefore, the recurrent inhibition should be sufficiently strong to result in a balanced state. Our observations indicate that this is the case for $K \bar{v}>1000 \mathrm{~Hz}$ (Fig. 4.2G).

In excitatory-inhibitory cLIF networks we chose the coupling strengths according to Eq. 2.31) to yield identical firing statistics as in inhibitory networks. This enables a quantitative comparison of the two types of networks. Consequently, the balanced state in excitatory-inhibitory cLIF networks emerged broadly in the entire parameter range and with the expected asynchronous irregular firing statistics (Fig. 4.3). The observed phase transition to a synchronous regular state with increasing excitatory-inhibitory feedback loop strength $\varepsilon$ in theta neuron networks did not occur in cLIF networks. This agrees with the observation in rapid theta neuron networks, where this phase transition already disappeared for moderately large AP onset rapidness, because LIF neurons correspond to an infinite AP onset rapidness.

\subsection{Dynamics of Inhibitory Networks}

Although we are studying a completely different neuron and synapse model in this chapter, a unique shape of the Lyapunov spectra that is independent of the network size $N$ also characterizes the dynamics of cLIF networks in the balanced state and implies extensive dynamics (Fig. 4.4). The balanced state in such networks is again characterized by a decreasing synchrony measure $\chi \sim 1 / \sqrt{N}$ and a high network size-independent coefficient of variation cv (Fig. 4.4A,B). Because each neuron contributes two dimensions (current and voltage) to the networks phase space, $2 \mathrm{~N}$ Lyapunov exponents now describe the network dynamics of cLIF neurons. The complete spectra of Lyapunov exponents for inhibitory networks are displayed in Fig. 4.4 C. Compared to the Lyapunov spectra in Chapters 2 and 3, the spectra of cLIF networks discussed here exhibit two main differences: (i) The smallest Lyapunov exponent $\lambda_{\min }$ was isolated from the rest of the Lyapunov exponents, (ii) the Lyapunov spectra exhibited two distinct branches. The largest Lyapunov exponent was positive and did not seem to saturate with increasing network size $N$, even though it increased very slowly as $\lambda_{\max } \sim \log N$ (Fig. 4.4D). Nevertheless, with increasing network size the complete spectrum of Lyapunov exponents converged to a unique shape. This is indicated by the inset in Fig. 4.4 C and the fact that the entropy production rate $H$, Eq. (1.5), and the attractor dimension $D$, Eq. (1.6), increased linearly with the number of neurons $N$ (Fig. 4.4F,G). Thus the 

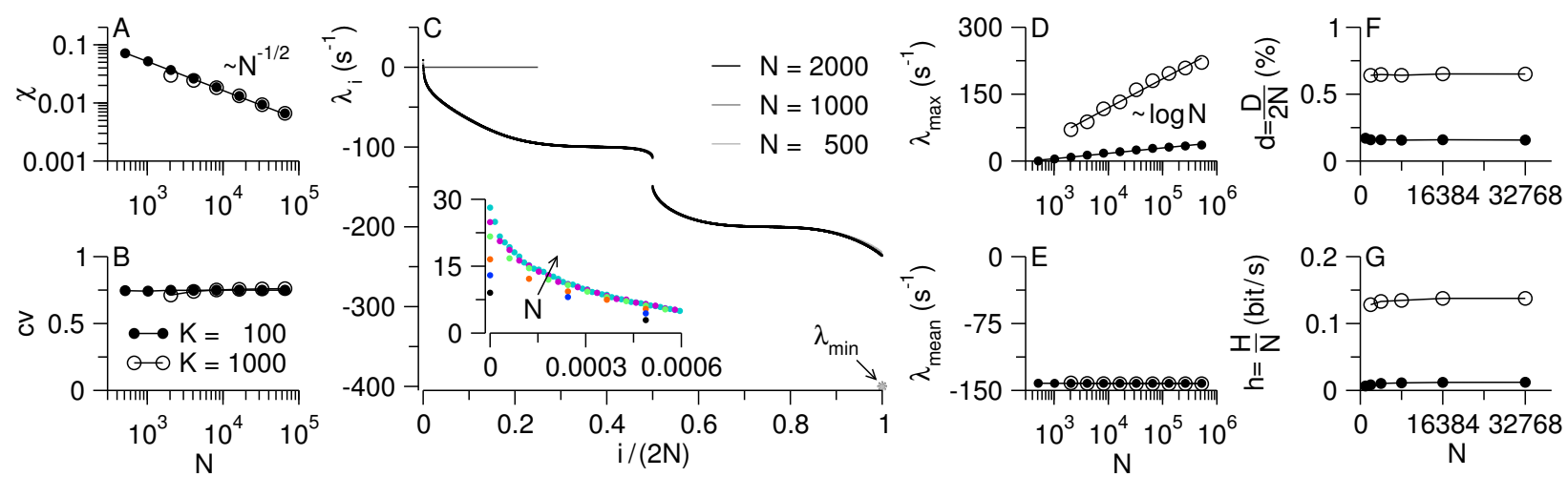

Figure 4.4 - Extensive dynamics in balanced inhibitory networks of $N$ correlated integrate and fire neurons. (A) Synchrony measure $\chi$ (straight line: $\chi \sim 1 / \sqrt{N}$ ), (B) average coefficient of variation $\mathrm{cv},(\mathrm{C})$ full spectra of Lyapunov exponents $\left\{\lambda_{i}\right\}$ for $K=100$ (inset: first few Lyapunov exponent for $N=2048 \ldots 65536$ ), (D) largest Lyapunov exponent $\lambda_{\max }=\lambda_{1}$ (straight lines: $\lambda_{\max } \sim \log N$ ), (E) mean Lyapunov exponent $\lambda_{\text {mean }}=\frac{1}{N} \sum_{i} \lambda_{i}$ (dotted line from random matrix approximation, Eq. (4.36) ), (F) information dimension in percent of phase space dimension $d=D /(2 N),(\mathrm{G})$ average entropy production rate per neuron per spike $h=H /(N \bar{v})$, (parameters: $N=10000, \bar{v}=10 \mathrm{~Hz}, \tau_{\mathrm{m}}=10 \mathrm{~ms}, \tau_{\mathrm{I}}=5 \mathrm{~ms}$; averages of 10 runs with different network realizations).

introduction of the relative attractor dimension $d=D /(2 N)$ and average entropy production rate per neuron $h=H / N$ as in the previous chapters is well-justified. One might speculate that such extensive dynamics is the general case in random neural network in the balanced state.

Positive and finite Lyapunov exponents indicating deterministic chaos in inhibitory cLIF networks are in fact a surprising result, because LIF networks with $\delta$ pulse coupling exhibit stable dynamics despite the irregular spiking activity [4-7]. This is called stable chaos. The Lyapunov spectra of LIF networks with $\delta$ pulse coupling are negative definite (see dashed line in Fig. 4.5C and Chapter 5). With finite synaptic time constants $\tau_{\mathrm{I}}$, the Lyapunov spectra were different, such that the largest Lyapunov exponent, attractor dimension and entropy production rate were positive, hence the dynamics chaotic. The Lyapunov spectra split into two branches. These two branches are related to the inverse of the two time constants - the membrane time constant $\tau_{\mathrm{m}}$ and the synaptic time constant $\tau_{\mathrm{I}}$ (indicated by the dotted and dashed-dotted lines in Fig. $4.5 \mathrm{C}$, respectively). This is also supported by the fact that in the random matrix approximation, Eq. (4.36), the mean Lyapunov exponent is approximated by the inverse of the harmonic mean of $\tau_{\mathrm{I}}$ and $\tau_{\mathrm{m}}$. Indeed, the random matrix approximation of the mean Lyapunov exponent agrees excellently with the numerical results (Fig. 4.5E).

Although chaotic, the entropy production rate in cLIF networks was much lower than for example in theta neuron networks (see Chapter 2). The dynamics of cLIF networks were hardly sensitive to a variation of the synaptic coupling strength $J_{0}$ despite an increased spike variability indicated by an increased coefficient of variation (Fig. 4.6). With an increasing number of synaptic inputs $K$ the largest Lyapunov exponent, attractor dimension and entropy production rate increased and converged in the large $K$-limit (Fig. 4.7). This also holds for an increase of the average firing rate $\bar{v}$. It should be noted here that the networks with $K=100$ and $\bar{v}<10 \mathrm{~Hz}$, which exhibit negative definite Lyapunov spectra, are not in a balanced state as discussed above. It can be seen in Fig. 4.8 C that the first part of the Lyapunov spectra of the cLIF networks ( $\tau_{\mathrm{I}}=5 \mathrm{~ms}$ ) for $K=100$ and $\bar{v}=1 \mathrm{~Hz}$ overlaps with the spectra of LIF networks ( $\delta$ pulses). Increasing the average firing rate towards the balanced state led to a considerable change of the Lyapunov spectra of cLIF 


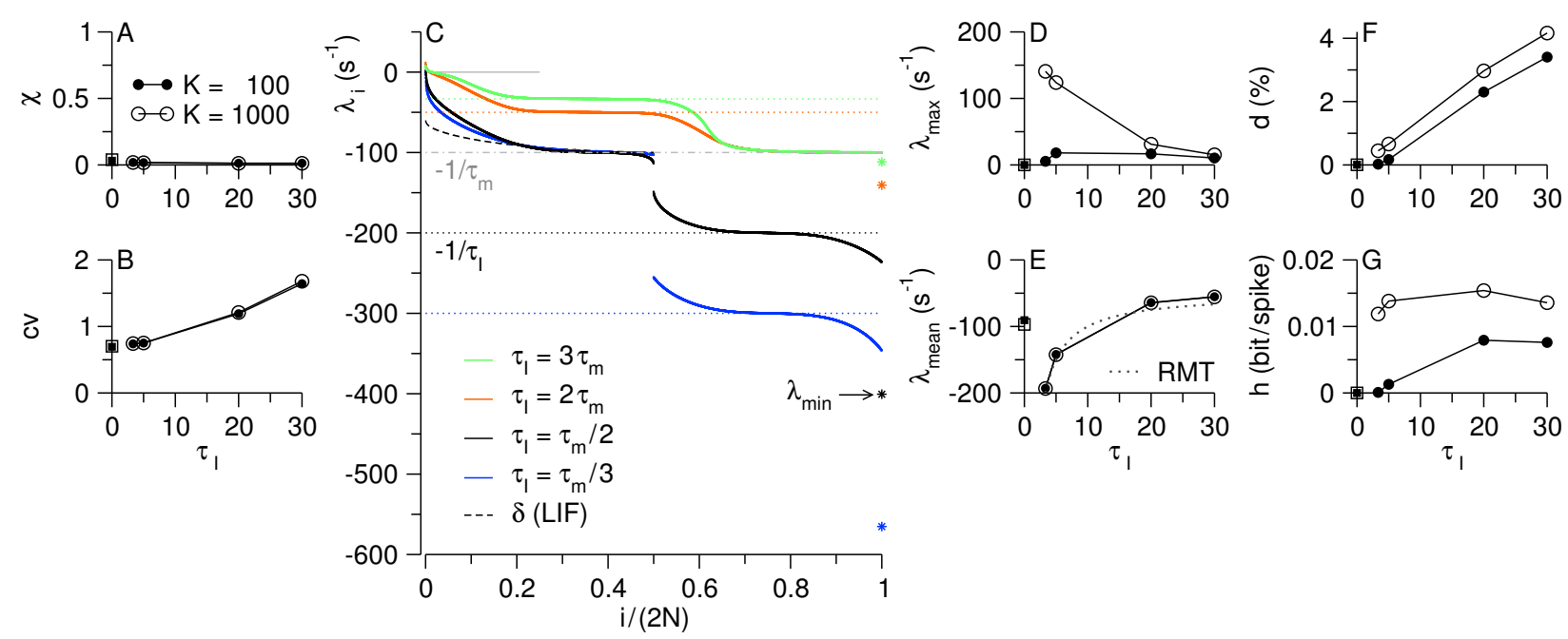

Figure 4.5 - Dynamics for different synaptic time constants $\tau_{\mathrm{I}}$ and $\delta$ pulse coupling (LIF). (A) Synchrony measure $\chi$, (B) average coefficient of variation $\mathrm{cv}$, (C) full spectra of Lyapunov exponents $\left\{\lambda_{i}\right\}$ (for $N=2000, K=100$ ), (D) largest Lyapunov exponent $\lambda_{\max }=\lambda_{1}$, (E) mean Lyapunov exponent $\lambda_{\text {mean }}=\frac{1}{N} \sum_{i} \lambda_{i}$ (dotted line from random matrix approximation, Eq. 4.36), (F) information dimension in percent of phase space dimension $d=D /(2 N),(\mathrm{G})$ average entropy production rate per neuron per spike $h=H /(N \bar{v})$, (parameters: $N=10000, \bar{v}=10 \mathrm{~Hz}, \tau_{\mathrm{m}}=10 \mathrm{~ms}$; averages of 10 runs with different network realizations; squares at $\tau_{\mathrm{I}}=0$ results are from leaky integrate and fire (LIF) networks with $\delta$ pulse coupling).
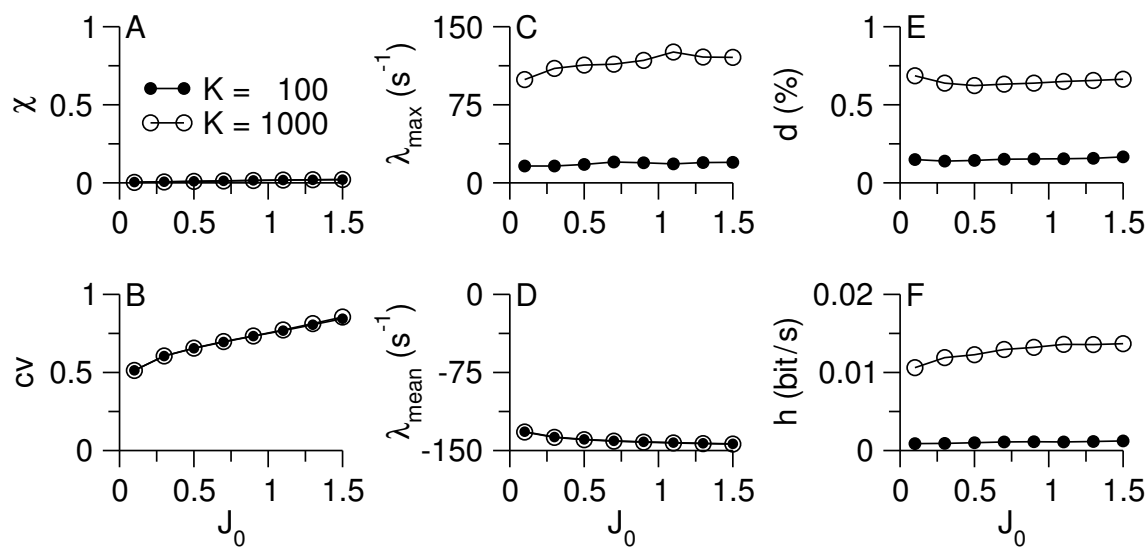

Figure 4.6 - Dynamics for varied coupling strength $J_{0}$. (A) Synchrony measure $\chi$, (B) average coefficient of variation cv, (C) largest Lyapunov exponent $\lambda_{\max }=\lambda_{1}$, (D) mean Lyapunov exponent $\lambda_{\text {mean }}=\frac{1}{N} \sum_{i} \lambda_{i},(\mathrm{E})$ information dimension in percent of phase space dimension $d=D /(2 N)$, (F) average entropy production rate per neuron per spike $h=H /(N \bar{v})$, (parameters: $N=10000, \bar{v}=10 \mathrm{~Hz}, \tau_{\mathrm{m}}=10 \mathrm{~ms}, \tau_{\mathrm{I}}=5 \mathrm{~ms}$; averages of 10 runs with different network realizations). 

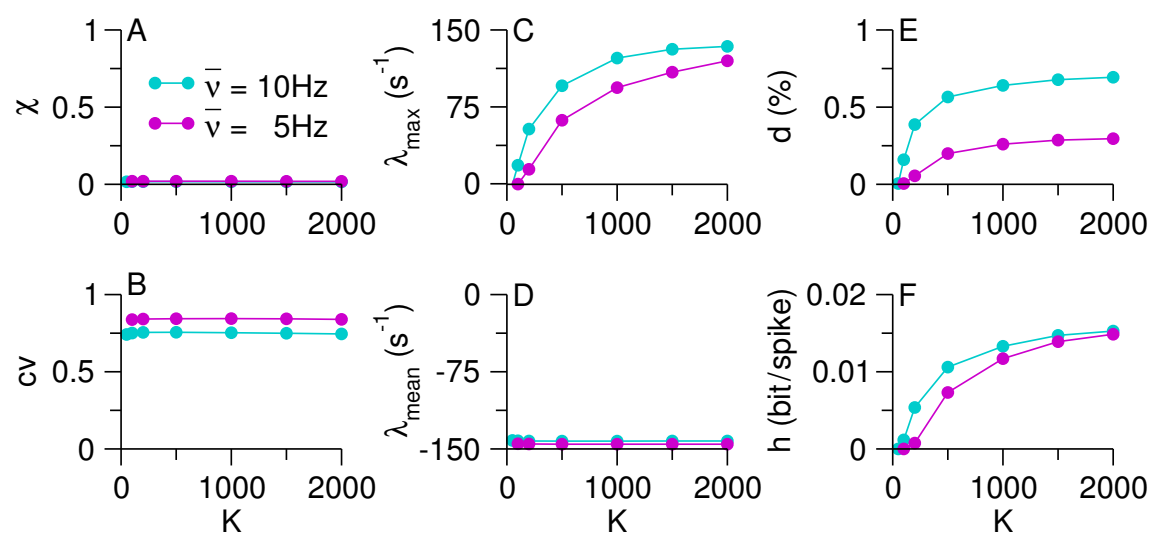

Figure 4.7 - Dynamics for varied number of synapses per neuron $K$. (see Fig. 4.6 for description, $\bar{v}=10 \mathrm{~Hz})$.

networks such that the largest Lyapunov exponent was positive for $\bar{v}>6 \mathrm{~Hz}$. However, for large $K=1000$ the largest Lyapunov exponent and the entropy production rate converged and the attractor dimension increased with the average firing rate. These results imply that the overall rate of entropy production due to the temporal synaptic transmission does not exceed a value of 0.02 bit per spike per neuron and was thus orders of magnitudes lower than in theta neuron networks. Compared to rapid theta neuron networks, the very low entropy production rate in cLIF networks would only be comparable in magnitude very close to the transition to stability-the edge of chaos.

\subsection{Dynamics of Excitatory-Inhibitory Networks}

Similar to the results in Chapters 2 and 3 , the chaotic dynamics of cLIF networks intensified with the activation of excitatory-inhibitory feedback loops (Fig. 4.9). Due to the specific parametrization of the coupling matrix (2.51), the asynchronous and irregular firing statistics were insensitive to the activation of the excitatory-inhibitory feedback loops $\varepsilon$ (Fig. 4.9A,B). The Lyapunov spectra of excitatory-inhibitory cLIF networks changed in a way that the largest Lyapunov exponent, attractor dimension and entropy production rate increased with $\varepsilon$ (Fig. 4.9 C-G). The entropy production rate, however did not increase by orders of magnitude such that the observation of a much lower entropy production rate in CLIF networks compared to theta neuron networks holds in excitatory-inhibitory networks as well.

The Lyapunov spectra showed the particular splitting into two branches as observed in inhibitory networks before. We varied the synaptic time constant $\tau_{\mathrm{I}}$ at a fixed excitatory-inhibitory feedback loop strength (Fig. 4.10). The effect of the synaptic time constant is identical with that in inhibitory networks, which strengthens the observation in Chapter 2 and 3 that inhibitory networks already capture the dynamics of excitatory-inhibitory networks quite well.

The precise quantification of the chaos in cLIF networks, however, depends on the specific networks' parameters, including the number of excitatory neurons (Fig. 4.11). It occurs that at fixed excitatory-inhibitory feedback loop strength, an increased number of excitatory neurons reduced the chaos in the networks. The reduction converges and implies an optimal value in terms of reduced entropy production at $N_{E} / N_{I}=4$, which surprisingly agrees with the realistic ratio of excitatory and inhibitory neurons in cortical networks [95]. 

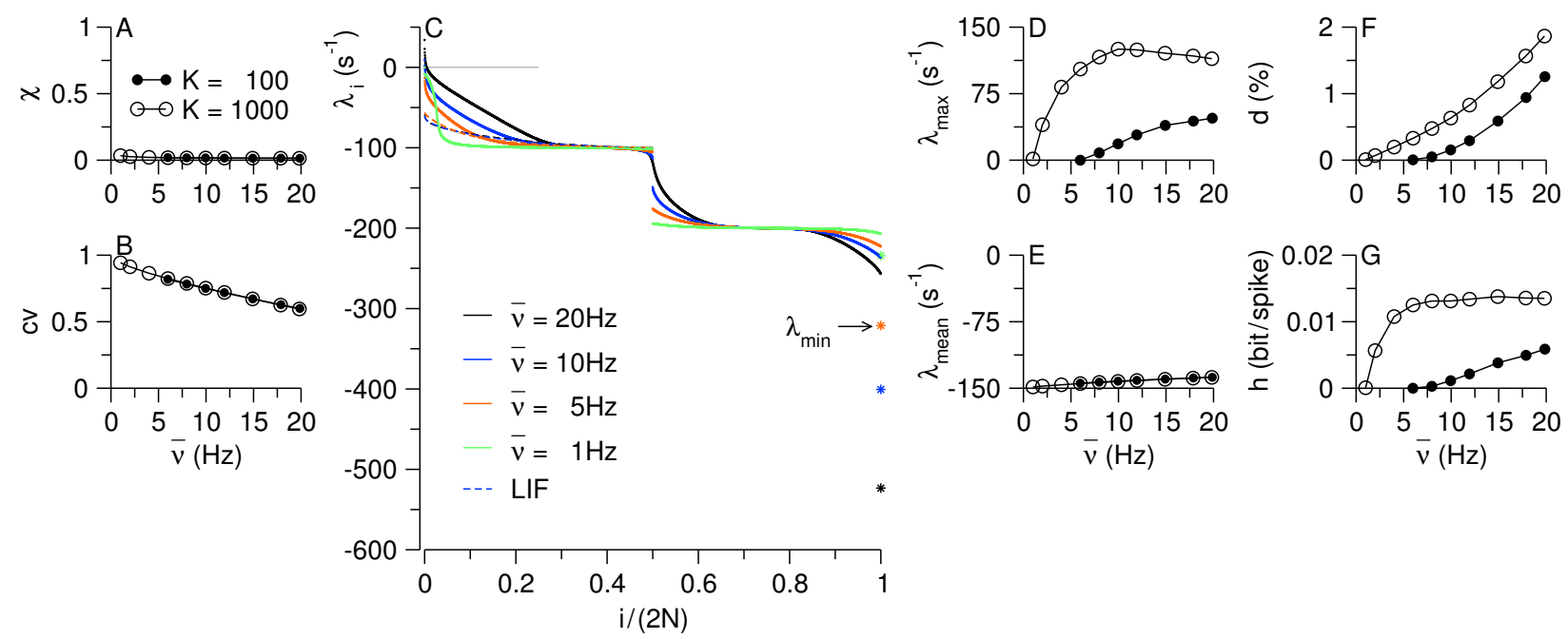

Figure 4.8 - Dynamics for varied firing rate $\bar{v}$ : (A) Synchrony measure $\chi$, (B) average coefficient of variation cv, (C) full spectra of Lyapunov exponents $\left\{\lambda_{i}\right\}$ (for $N=2000, K=100$ ), (D) largest Lyapunov exponent $\lambda_{\max }=\lambda_{1}$, (E) mean Lyapunov exponent $\lambda_{\text {mean }}=\frac{1}{N} \sum_{i} \lambda_{i}$, (F) information dimension in percent of phase space dimension $d=D /(2 N),(\mathrm{G})$ average entropy production rate per neuron per spike $h=H /(N \bar{v})$, (parameters: $N=10000, \tau_{\mathrm{m}}=10 \mathrm{~ms}$; averages of 10 runs with different network realizations).
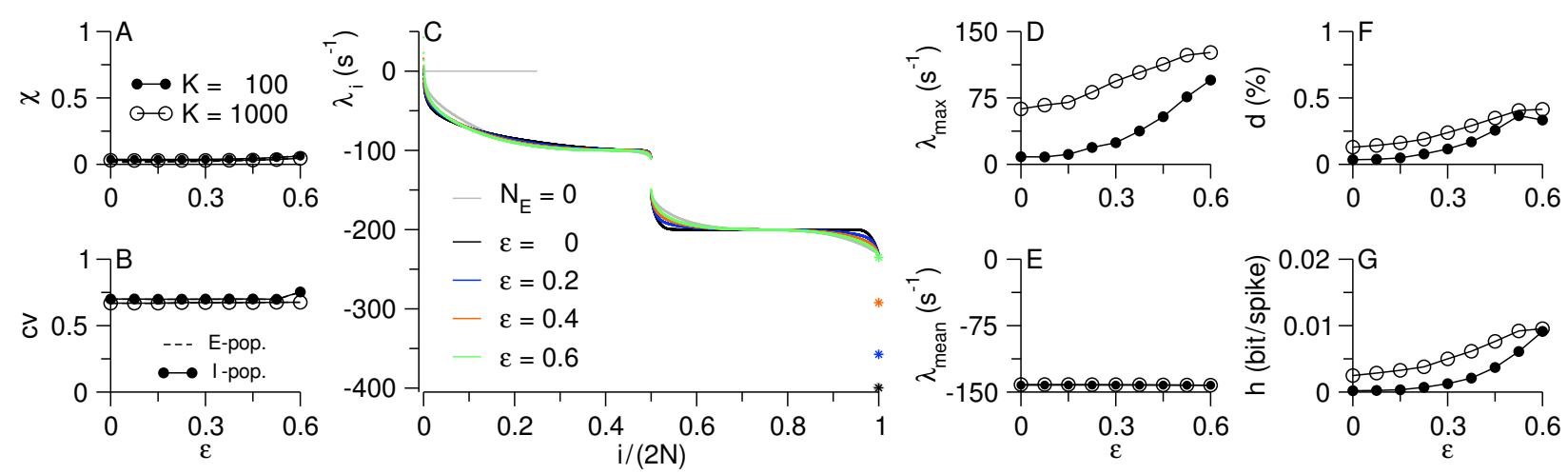

Figure 4.9 - Dynamics of balanced excitatory-inhibitory networks while activating the excitatoryinhibitory feedback loops with $\varepsilon$. (A) Synchrony measure $\chi$, (B) average coefficient of variation $\mathrm{cv},(\mathrm{C})$ full spectra of Lyapunov exponents $\left\{\lambda_{i}\right\}$ (for $N=2000, K=100$ ), (D) largest Lyapunov exponent $\lambda_{\max }=\lambda_{1}$, (E) mean Lyapunov exponent $\lambda_{\text {mean }}=\frac{1}{N} \sum_{i} \lambda_{i}$ (dotted line from random matrix approximation, Eq. 4.36), (F) information dimension in percent of phase space dimension $d=D /(2 N),(\mathrm{G})$ average entropy production rate per neuron per spike $h=H /(N \bar{v})$, (parameters: $N_{E}=4 N_{I}, N=10000, \bar{v}=10 \mathrm{~Hz}, \tau_{\mathrm{m}}=10 \mathrm{~ms}, \tau_{\mathrm{I}}=$ $5 \mathrm{~ms}, \eta=0.9$; averages of 10 runs with different network realizations). 


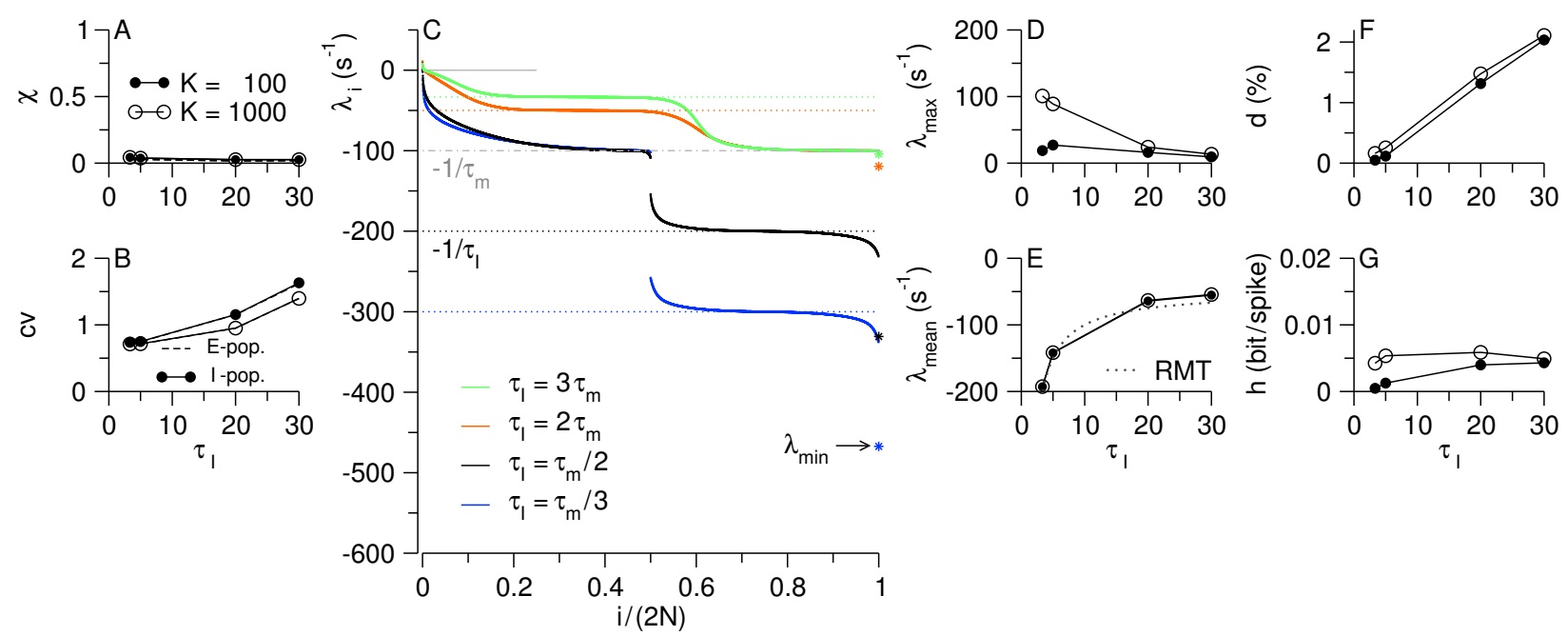

Figure 4.10 - Dynamics for different synaptic time constants $\tau_{\mathrm{I}}$. (see Fig. 4.9 for description, $\varepsilon=0.3$ ).
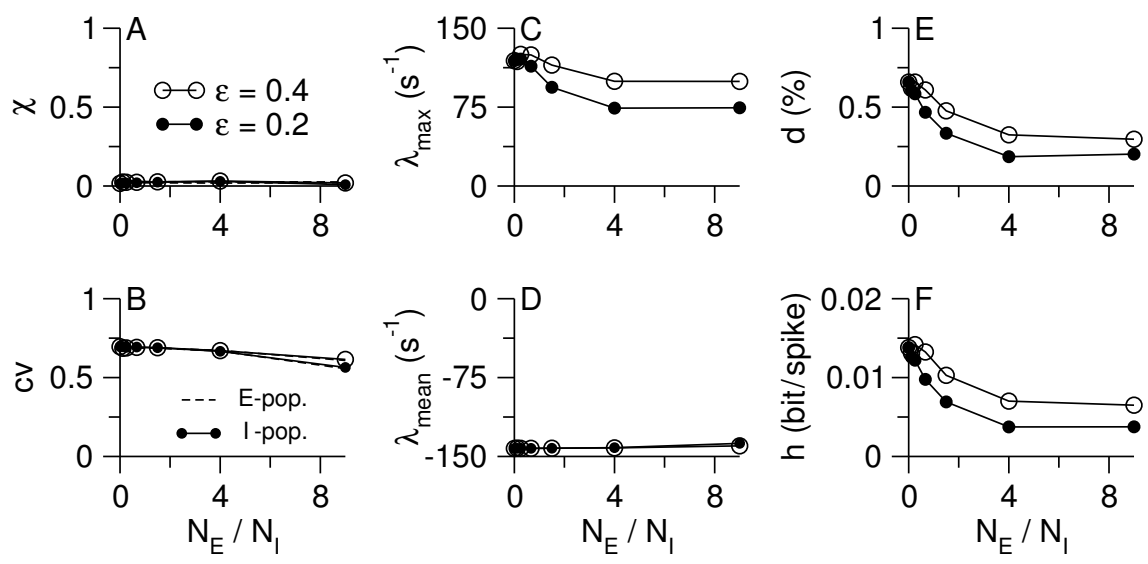

Figure 4.11 - Dynamics for varied ratio of the number of excitatory to inhibitory neurons $N_{E} / N_{I}$ : (A) Synchrony measure $\chi,(B)$ average coefficient of variation cv, (C) largest Lyapunov exponent $\lambda_{\max }=\lambda_{1}$, (D) mean Lyapunov exponent $\lambda_{\text {mean }}=\frac{1}{N} \sum_{i} \lambda_{i}$, (E) information dimension in percent of phase space dimension $d=D /(2 N),(\mathrm{F})$ average entropy production rate per neuron per spike $h=H /(N \bar{v})$, (parameters: $N=$ $10000, K=1000, \bar{v}=10 \mathrm{~Hz}, \tau_{\mathrm{m}}=10 \mathrm{~ms}, \tau_{\mathrm{I}}=5 \mathrm{~ms}, \eta=0.9$; averages of 10 runs with different network realizations). 


\subsection{Summary}

In this chapter we investigated the influence of temporal synaptic transmission on the collective network dynamics. Because the approach introduced in Chapter 2 is based on one-dimensional $\delta$ pulse coupled neurons, it was here extended to the necessary two-dimensional neuron model. This also illustrates possible extensions to other exactly solvable similar neuron models. Here, we started from the exactly solvable leaky integrate and fire (LIF) model. Networks of LIF neurons with inhibitory $\delta$ pulse coupling exhibit stable dynamics. It is therefore an ideal candidate to study the effect of temporally extended postsynaptic currents.

The results presented in this chapter reveal that LIF networks with exponentially decaying postsynaptic currents exhibit:

- Deterministic chaos in the balanced state (the observed transition from stable to chaotic dynamics coincides with the transition from unbalanced to balanced networks).

- Extensive dynamics, indicated by a unique shape of the Lyapunov spectrum in the large network limit and linearly increasing number of positive Lyapunov exponents, attractor dimension and entropy production rate with increasing number of neurons.

- Low dimensional attractors, at most $1 \%$ of the phase space dimension, and two orders of magnitude lower than in comparable theta neuron networks.

- Low entropy production rate, at most 0.02 bit per spike per neuron, and two orders of magnitude lower than in comparable theta neuron networks.

- Activation of excitatory-inhibitory feedback loops intensifies the chaos and renders the dynamics of LIF networks chaotic in general.

The observed chaotic dynamics in inhibitory networks of balanced LIF networks is entirely a consequence of the incorporation of temporal synaptic transmission. Equivalent LIF networks with $\delta$ pulse coupling have been shown to exhibit stable dynamics with respect to infinitesimal perturbations. The fragility of this stable dynamics to temporal synaptic transmission raises the question how fragile this stability is in general. 


\section{Stable Chaos in Networks of Leaky Integrate and Fire Neurons}

Instantaneous Action Potential Generation

In this chapter, we will continue to study $\delta$ pulse synaptic coupling, for which inhibitory leaky integrate and fire (LIF) networks exhibit stable chaos [4-7]. This form of dynamics, originally discovered in coupled map lattices [35], is characterized by stable dynamics despite an erratic network activity. The result of stable dynamics in inhibitory networks of LIF neurons in the balanced state is in contrast to the dynamics of binary neuron networks, found to exhibit an extremely strong form of chaos with an infinite Lyapunov exponent [2,3]. Both neuron models in these studies are highly simplified descriptions of the single neuron dynamics.

The binary neuron model is a discrete model in which neurons can either be active or inactive. It originates from the analogy of spins with the states up and down allowing for the application of meanfield theory developed in spin glass theory. The authors in Ref. [3] suspected that the infinite Lyapunov exponent in their analysis was a results of the discreteness of the binary neuron model as a consequence of an infinite gain of the smallest perturbations, but they expected the balanced state to be generally chaotic.

The LIF model is a continuous neuron model with spikes being virtually emitted when the membrane potential crosses a threshold and then reset to the resting potential. It thus neglects the active AP generation of real neurons. The special case of inhibitory LIF networks as models of cortical circuits is interesting because one can then study the effect of different mechanisms that destabilize the dynamics.

In Chapter 4 it was demonstrated that temporal synaptic coupling is one mechanism that destabilizes the collective dynamics of LIF neurons. We have shown that such cLIF networks exhibit chaotic dynamics in the balanced state, although the strength of this form of chaos was much lower than when incorporating the single neuron instability leading to an active AP generation.

In Chapters 2 and 3 we demonstrated the strong influence of active AP generation in the dynamical nature of neural networks. The simplest neuron model incorporating an active AP generation is the theta neuron model. Inhibitory networks of theta neurons in the balanced state generally exhibit chaotic dynamics (Chapter 2). A shift of the membrane potential distribution of the neurons when they were the most sensitive to perturbations towards the AP generating instability suggested that this feature of single neuron dynamics renders the collective dynamics of balanced networks robustly chaotic. Networks of rapid theta neurons revealed a transition of the dynamical nature from chaotic to stable upon increasing the AP onset rapidness $r$ (Chapter 3). In the limit $r \rightarrow \infty$, corresponding to an instantaneous AP initiation, finite inhibitory networks exhibit stable dynamics. The limit of $r \rightarrow \infty$ is related to the leaky integrate and fire (LIF) model, in which a dynamic AP generation is neglected but the spike is virtually instantaneously emitted when the membrane po- 
tential crosses a threshold. Because the LIF model is widely used in computational neuroscience and because it corresponds to the case of infinite AP onset rapidness, it is important to further understand the collective dynamics of this special neuron model.

In the first section of this chapter, we will derive the necessary exact closed form expressions of the LIF model to directly apply the approach developed in Chapter 2. This will allow for numerically exact simulations and the calculation of the full Lyapunov spectra of LIF networks completed with a random matrix approximation of the mean Lyapunov exponent. The analysis of the collective network dynamics is in this chapter extended to the firing rate response of the networks to single spike failures and distance measurement between such a perturbed trajectory and its reference trajectory. This reveals the occurrence of a positive pseudo Lyapunov exponent, in contrast to negative definite Lyapunov spectra. The exploration of the evolution of finite size perturbations then reveals an exotic phase space structure of exponentially separating flux tubes around unique stable trajectories consistent with the two seemingly contradictory observations. These results are currently under review at Physical Review Letters. In the last section of this chapter we will draw the connection to neurons with finite AP onset rapidness.

\subsection{Leaky Integrate and Fire Model}

We studied the dynamics of the balanced state in inhibitory networks of $N$ leaky integrate and fire (LIF) neurons following the approach introduced in Chapter 2. The neurons membrane potentials $V_{i} \in\left(-\infty, V_{\mathrm{T}}\right)$ with $i=1 \ldots N$ follow the differential equation (2.1) with a linear term $F\left(V_{i}\right)=-V_{i}$, thus

$$
\tau_{\mathrm{m}} \frac{\mathrm{d} V_{i}(t)}{\mathrm{d} t}=-V_{i}(t)+I_{i}(t)
$$

This is complemented with the condition that whenever the membrane potential $V_{i}$ reaches the threshold $V_{\mathrm{T}} \equiv 1$, the neuron is said to emit a spike and $V_{i}$ is reset to $V_{\mathrm{R}} \equiv 0$. The leaky integrate and fire model has an intrinsic rheobase current $I_{T} \equiv V_{T}$. This is the minimally required input current for tonic firing. The neurons integrate the synaptic input current $(2.2)$ :

$$
I_{i}(t)=I_{T}+\sqrt{K} I_{0}-\frac{J_{0} \tau_{\mathrm{m}}}{\sqrt{K}} \sum_{j \in \operatorname{pre}(i)} \sum_{s} \delta\left(t-t_{j}^{(s)}\right),
$$

composed of constant external excitatory currents $\sqrt{K} I_{0}$ and nondelayed inhibitory $\delta$ pulses of strength $-J_{0} / \sqrt{K}$ received at the spike times $\left\{t_{j}^{(s)}\right\}$ of the presynaptic neurons $j \in \operatorname{pre}(i)$. The neurons were arranged on directed sparse Erdös-Rényi random graphs of fixed mean indegree $K$. Thus, all neurons are connected to $K$ presynaptic neurons on average. At the spike times of the presynaptic neurons, the membrane potential of the spike receiving neuron immediately changed by an amount $-J_{0} / \sqrt{K}$. The resulting recurrent inhibition balanced the constant external excitation in the networks leading to a balanced state (see Section 2.4).

With the exact solutions of the leaky integrate and fire model we form the iterative map, Eq. 2.3. Between successive spikes events $t_{s}$ and $t_{s+1}$, the neurons integrate the constant current $I_{c}=$ $I_{T}+\sqrt{K} I_{0}$ and the membrane potentials follow the solution of Eq. 5.1) with $I_{i}(t) \equiv I_{c}$. This solution is given by

$$
f\left(V_{i}\left(t_{s}\right), t_{s+1}-t_{s}\right) \equiv V_{i}\left(t_{s+1}\right)=I_{c}-\left(I_{c}-V_{i}\left(t_{s}\right)\right) \mathrm{e}^{-\left(t_{s+1}-t_{s}\right) / \tau_{\mathrm{m}}} .
$$


Receiving a $\delta$-pulse at the spike time of a presynaptic neuron leads to a step-like change of the membrane potential

$$
g\left(V_{i^{*}}\left(t_{s+1}^{-}\right)\right) \equiv V_{i^{*}}\left(t_{s+1}^{+}\right)=V_{i^{*}}\left(t_{s+1}^{-}\right)-\frac{J_{0}}{\sqrt{K}},
$$

where $t_{s}^{\mp}$ denotes time just before/after the spike reception.

More convenient, however, is the phase representation with constant phase velocity and the phase-transition curve of the LIF neurons. In this representation, the phases $\phi_{i} \in(-\infty, 1)$ describe the states of the neurons relative to their unperturbed free-running interspike intervals, which is obtained from Eq. (5.3) as the time between reset and threshold without any inhibitory inputs. The free interspike intervals are

$$
\begin{aligned}
T_{i}^{\text {free }} & =-\tau_{\mathrm{m}} \ln \left(\frac{V_{T}-I_{c}}{V_{R}-I_{c}}\right) \\
& =\tau_{\mathrm{m}} \ln \left(\frac{I_{c}}{I_{c}-1}\right) \\
& =\tau_{\mathrm{m}} \ln \left(1+\frac{1}{\sqrt{K} I_{0}}\right)
\end{aligned}
$$

In the large $K$-limit the free interspike interval $(5.5)$ becomes $T_{i}^{\text {free }} \stackrel{K \rightarrow \infty}{\simeq} \frac{\tau_{\mathrm{m}}}{\sqrt{K} I_{0}}+\mathscr{O}\left(\frac{1}{K}\right)$. The phase of the neuron, describing its state relative to the unperturbed cycle then reads

$$
\begin{aligned}
\phi_{i} & =-\frac{\tau_{\mathrm{m}}}{T_{i}^{\text {free }}} \ln \left(\frac{V-I_{c}}{V_{R}-I_{c}}\right) \\
& =\frac{\tau_{\mathrm{m}}}{T_{i}^{\text {free }}} \ln \left(\frac{I_{c}}{I_{c}-V}\right)
\end{aligned}
$$

and the reverse transformation

$$
V_{i}=I_{c}\left(1-\exp \left(-\phi_{i} T_{i}^{\mathrm{free}} / \tau_{\mathrm{m}}\right)\right) .
$$

In the large $K$-limit, the transformations (5.8) and (5.9) become $\phi_{i} \stackrel{K \rightarrow \infty}{\simeq} V_{i}+\mathscr{O}\left(\frac{1}{\sqrt{K}}\right)$.

Thus, between successive spikes events $t_{s}$ and $t_{s+1}$, the neurons' phases evolve with constant phase velocity $1 / T_{i}^{\text {free }}$ and the phase evolution reads

$$
f\left(\phi_{i}\left(t_{s}\right), t_{s+1}-t_{s}\right) \equiv \phi_{i}\left(t_{s+1}^{-}\right)=\phi_{i}\left(t_{s}\right)+\left(t_{s+1}-t_{s}\right) / T_{i}^{\text {free }}
$$

When receiving a spike, the step-like change in the membrane potential transforms in the phase representation to the phase-transition curve

$$
g\left(\phi_{i^{*}}\left(t_{s+1}^{-}\right)\right) \equiv \phi_{i^{*}}\left(t_{s+1}\right)=-\frac{\tau_{\mathrm{m}}}{T_{i^{*}}^{\text {free }}} \ln \left(\exp \left(-\phi_{i^{*}}\left(t_{s+1}^{-}\right) T_{i^{*}}^{\text {free }} / \tau_{\mathrm{m}}\right)+\frac{J_{0}}{\sqrt{K} I_{c}}\right) .
$$


Hence, the phase-response curve of the LIF model is

$$
\begin{aligned}
Z\left(\phi_{i^{*}}\left(t_{s+1}^{-}\right)\right) & =g\left(\phi_{i^{*}}\left(t_{s+1}^{-}\right)\right)-\phi_{i^{*}}\left(t_{s+1}^{-}\right) \\
& =-\frac{\tau_{\mathrm{m}}}{T_{i^{*}}^{\text {free }}} \ln \left(\exp \left(-\phi_{i^{*}}\left(t_{s+1}^{-}\right) T_{i^{*}}^{\text {free }} / \tau_{\mathrm{m}}\right)+\frac{J_{0}}{\sqrt{K} I_{c}}\right)-\phi_{i^{*}}\left(t_{s+1}^{-}\right) .
\end{aligned}
$$

In the large $K$-limit, the phase-response curve becomes

$$
\begin{aligned}
Z(\phi) \stackrel{K \rightarrow \infty}{\simeq}-\frac{J_{0}}{\sqrt{K}}+\frac{J_{0}(0.5-\phi)}{I_{0} K}+\mathscr{O}\left(\frac{1}{K^{\frac{3}{2}}}\right) \\
\quad=-\frac{J_{0}}{\sqrt{K}}+\frac{0.5-\phi}{K \bar{v} \tau_{\mathrm{m}}}+\mathscr{O}\left(\frac{1}{K^{\frac{3}{2}}}\right),
\end{aligned}
$$

where the balance equation (2.19) was used in the last step. The derivative of the phase-response curve used for the single spike Jacobians reads

$$
Z^{\prime}\left(\phi_{i^{*}}\left(t_{s+1}^{-}\right)\right)=\frac{\exp \left(-\phi_{i^{*}}\left(t_{s+1}^{-}\right) T_{i}^{\text {free }} / \tau_{\mathrm{m}}\right)}{\exp \left(-\phi_{i^{*}}\left(t_{s+1}^{-}\right) T_{i}^{\text {free }} / \tau_{\mathrm{m}}\right)+J_{0} /\left(\sqrt{K} I_{c}\right)}-1 .
$$

In the large $K$-limit, the derivative of the phase-response curve becomes

$$
\begin{aligned}
Z^{\prime}(\phi) \stackrel{K \rightarrow \infty}{\simeq}-\frac{J_{0}}{I_{0} K}+\frac{J_{0}(1-\phi)}{I_{0}^{2} K^{\frac{3}{2}}}+\mathscr{O}\left(\frac{1}{K^{2}}\right) \\
\quad=-\frac{1}{K \bar{v} \tau_{\mathrm{m}}}+\frac{1-\phi}{\sqrt{K} I_{0} K \bar{v} \tau_{\mathrm{m}}}+\mathscr{O}\left(\frac{1}{K^{2}}\right)
\end{aligned}
$$

The last ingredient for true event-based simulations is an explicit equation for the time of the next spike occurring in the network, which is obtained from Eq. 5.10) with $\phi_{i}\left(t_{s+1}^{-}\right)=1$ :

$$
t_{s+1}=t_{s}+\min _{i}\left\{\left(1-\phi_{i}\left(t_{s}\right)\right) T_{i}^{\text {free }}\right\} .
$$

Altogether, with Eq. (5.10), (5.11) and (5.15), the iterative phase map, Eq. (2.14), is defined and the derivative of the phase-response curve, Eq. (5.14), yields the single spike Jacobians, Eq. 2.15). These are all prerequisites for true event-based simulations and the numerically exact calculation of the Lyapunov spectra. Before we present the results though, we adapt the derivation of the mean Lyapunov exponent in random matrix approximation from Section 2.5.1 for LIF networks.

\subsection{Mean Lyapunov Exponent}

We derived a random matrix approximation of the mean Lyapunov exponent $\bar{\lambda}=\frac{1}{N} \sum_{i=1}^{N} \lambda_{i}$ for theta neuron networks in Section 2.5.1. Analogously to this derivation, the mean Lyapunov exponent for homogeneous inhibitory LIF networks in the balanced state reads

$$
\bar{\lambda}=K \bar{v} \int \ln (d(V)) P(V) \mathrm{d} V
$$


with $d(V)$ denoting the derivative of the phase transition curve in the voltage representation. This derivative can be read off from Eq. (5.14) and the voltage-phase transformation, Eq. (5.8), written as $\exp \left(-\phi T^{\text {free }} / \tau_{\mathrm{m}}\right)=\left(I_{c}-V\right) / I_{c}$. The derivative then reads

$$
d(V)=\frac{I_{T}+\sqrt{K} I_{0}-V}{I_{T}+\sqrt{K} I_{0}-V+J_{0} / \sqrt{K}} .
$$

In the large $K$-limit the derivative becomes

$$
\begin{aligned}
& d(V) \stackrel{K \rightarrow \infty}{\simeq} 1-\frac{J_{0}}{I_{0} K}+\frac{J_{0}(1-V)}{I_{0}^{2} K^{\frac{3}{2}}}+\mathscr{O}\left(\frac{1}{K^{2}}\right) \\
& \approx 1-\frac{1}{K \bar{v} \tau_{\mathrm{m}}}+\frac{1-V}{\sqrt{K} I_{0} K \bar{v} \tau_{\mathrm{m}}}+\mathscr{O}\left(\frac{1}{K^{2}}\right),
\end{aligned}
$$

where the approximation of the average firing rate Eq. (2.19) was used in the last step. Eq. (5.17) inserted in the equation of the mean Lyapunov exponent in random matrix approximation (5.16) leads to

$$
\bar{\lambda} \stackrel{K \rightarrow \infty}{\simeq}-\frac{1}{\tau_{\mathrm{m}}}+\frac{1-\langle V\rangle}{\sqrt{K} I_{0} \tau_{\mathrm{m}}}+\mathscr{O}\left(\frac{1}{K}\right)
$$

Here, $\langle V\rangle=\int V P(V) \mathrm{d} V$ denotes the average of the membrane potential. Thus, in the large $K$-limit, the mean Lyapunov exponent becomes $\bar{\lambda} \approx-1 / \tau_{\mathrm{m}}$.

\subsubsection{Meanfield Approximation of the Membrane Potential Distribution}

The meanfield theory developed by Brunel and Hakim [100] for sparse networks in the asynchronous (balanced) state allows for the self-consistent calculation of the membrane potential distribution $P(V)$. We will use the membrane potential distribution to estimate the mean Lyapunov exponent, Eq. (5.16). The membrane potential distribution is given in Ref. [100] as

$$
\begin{aligned}
& P(V)=2 \frac{\bar{v}}{\sigma} \exp \left(-\frac{(V-\mu)^{2}}{\sigma^{2}}\right) \int_{\frac{V-\mu}{\sigma}}^{\frac{V_{T}-\mu}{\sigma}} \Theta\left(W-\frac{V_{R}-\mu}{\sigma}\right) \exp \left(W^{2}\right) \mathrm{d} W \\
& =\sqrt{\pi} \frac{\bar{v}}{\sigma} \exp \left(-\frac{(V-\mu)^{2}}{\sigma^{2}}\right) \begin{cases}\operatorname{erfi}\left(\frac{V_{T}-\mu}{\sigma}\right)-\operatorname{erfi}\left(\frac{V-\mu}{\sigma}\right) & V \geq 0 \\
\operatorname{erfi}\left(\frac{V_{T}-\mu}{\sigma}\right)-\operatorname{erfi}\left(\frac{V_{R}-\mu}{\sigma}\right) & V<0 .\end{cases}
\end{aligned}
$$

where we use for the magnitude of the input fluctuations $\sigma^{2} \approx J_{0}^{2} \bar{v} \tau_{\mathrm{m}}$ (Eq. (2.20) ) and for the mean input current $\mu \approx I_{T}+\sqrt{K}\left(I_{0}-J_{0} \bar{v} \tau_{\mathrm{m}}\right.$ ) (Eq. (2.19)). The integral was solved using the imaginary error function $\operatorname{erfi}(x)=\frac{\operatorname{erf}(t x)}{l}=\frac{2}{\sqrt{\pi}} \int_{0}^{x} \exp \left(t^{2}\right) \mathrm{d} t$. The parameters in Eq. (5.19) are $\bar{v}, K, I_{0}$ and $J_{0}$. We have set $J_{0}=1$, varied $\bar{v}$ and $K$ and chose $I_{0}$ selfconsistently such that the membrane potential distribution was normalized $\left(\int P(V) \mathrm{d} V=1\right)$. Self-consistency was checked with the equation of 

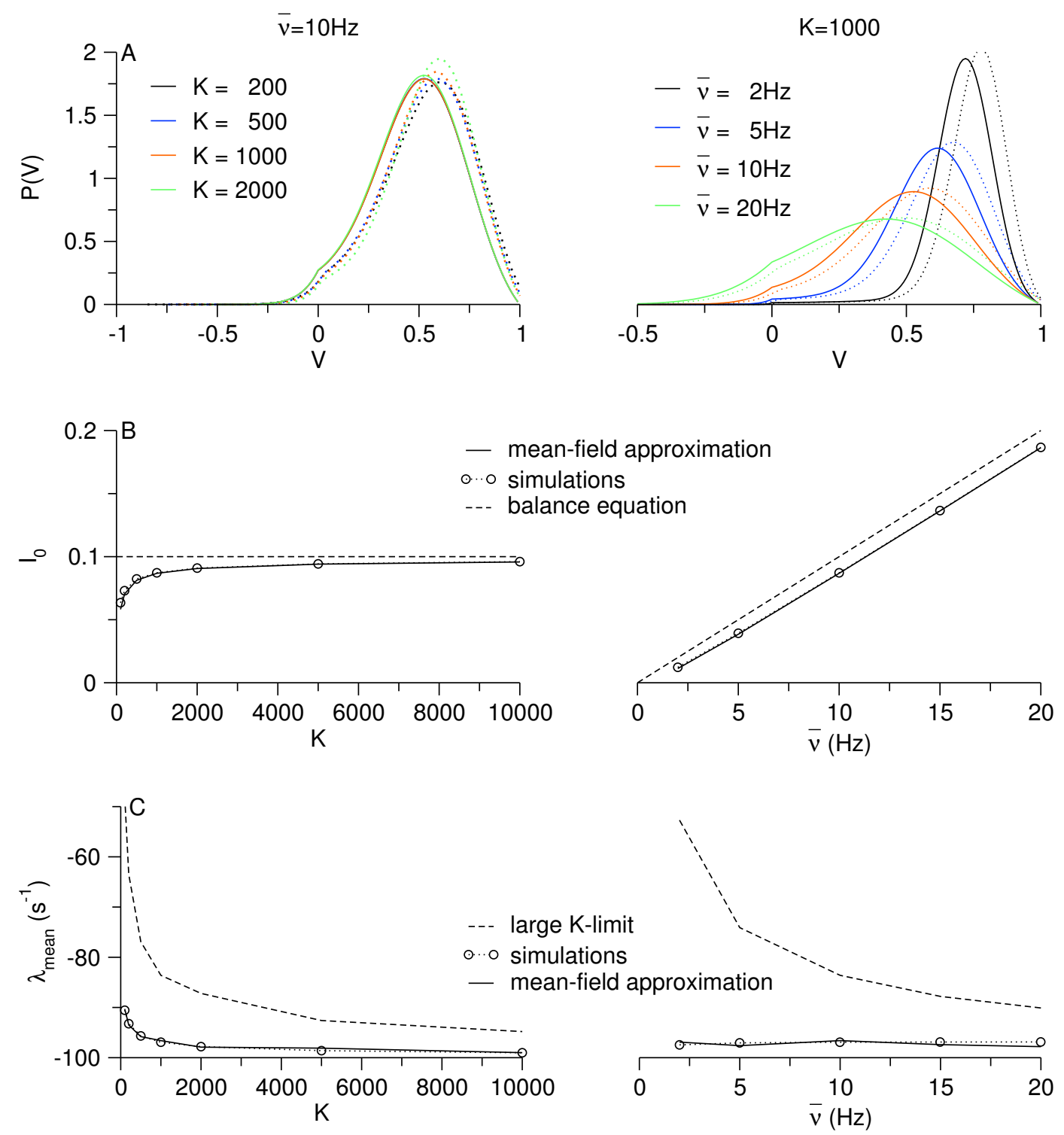

Figure 5.1 - Membrane potential distributions and mean Lyapunov exponents from meanfield approximation.

(A) Membrane potential distribution $P(V)$ and (B) external currents $I_{0}$ for different connectivities $K$ and average firing rates $\bar{v}$; straight lines: meanfield Eq. (5.19), dotted lines and open circles: simulations, dashed lines: balance equation Eq. (2.19),

(C) mean Lyapunov exponent $\bar{\lambda}$; straight lines: random matrix approximation Eq. 5.16 with membranepotential distributions from A, dotted lines and open circles: simulations, dash-dotted lines: large $K$ approximation Eq. (5.18), (parameters: $N=10000, K=1000, \bar{v}=10 \mathrm{~Hz}, J_{0}=1, \tau_{\mathrm{m}}=10 \mathrm{~ms}$ ). 

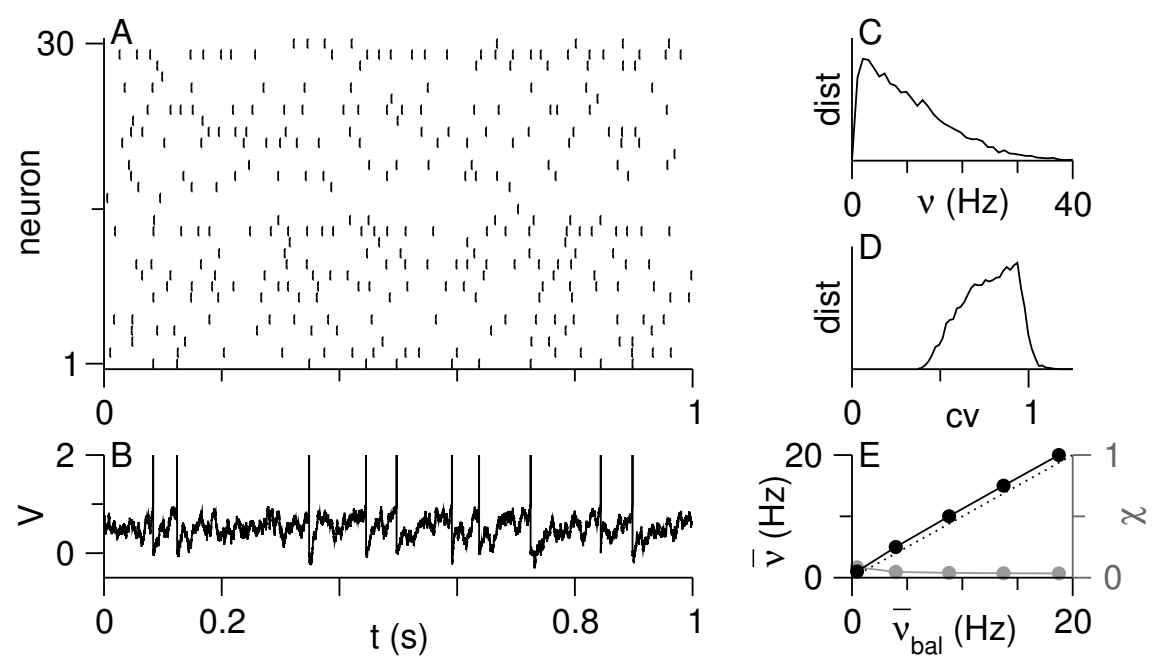

Figure 5.2 - The balanced state in inhibitory LIF networks. (A) Asynchronous irregular spike pattern of 30 randomly chosen neurons, (B) fluctuating voltage trace of one neuron (voltage increased to $V=2$ at spikes), (C,D) broad distributions of individual neurons' firing rates $v$ and coefficients of variation cv, (E) network-averaged firing rate $\bar{v}$ and synchrony measure $\chi$ versus predicted rate $\bar{v}_{\text {bal }}=I_{0} /\left(J_{0} \tau_{\mathrm{m}}\right)$ (dotted line: guide for the eye for $\bar{v}=\bar{v}_{\text {bal }}, \chi=\frac{\operatorname{STD}\left(\left[\phi_{i}\right]\right)}{\left[\operatorname{STD}\left(\phi_{i}\right)\right]}$ where $[\cdot]$ denotes population average), (parameters: $N=10000$, $\left.K=1000, \bar{v}=10 \mathrm{~Hz}, J_{0}=1, \tau_{\mathrm{m}}=10 \mathrm{~ms}\right)$.

the firing rate $\bar{v}$ provided in Ref. [100]

$$
\bar{v}=\left(\sqrt{\pi} \tau_{\mathrm{m}} \int_{\frac{V_{R}-\mu}{\sigma}}^{\frac{V_{T}-\mu}{\sigma}}(1+\operatorname{erf}(W)) \exp \left(W^{2}\right) \mathrm{d} W\right)^{-1}
$$

Representative membrane potential distributions and the external currents are displayed for various parameters in Fig. 5.1A. They agree very well with the simulations (dotted lines). The selfconsistent membrane potential distributions were then used to approximate the mean Lyapunov exponent, derived in the previous section. The results, displayed in Fig. 5.1TC, showed excellent agreement between the random matrix approximation (5.16) using the self-consistent membrane potential distribution (5.19) and our results from the simulations. The large $K$-limit appears quite valid for very large $K$, as expected.

The self-consistent membrane potential distributions were furthermore used to estimate the distance between uncorrelated states $D_{V}=\iint|V-W| P(V) P(W) \mathrm{d} V \mathrm{~d} W$, as given in Ref. [5]. In the phase representation this becomes

$$
D=\iint|\phi-\varphi| P(\phi) P(\varphi) \mathrm{d} \phi \mathrm{d} \varphi
$$

with $P(\phi)=P(V) I_{c} T^{\text {free }} \exp \left(-\phi T^{\text {free }}\right)$. The Eq. (5.19) of the distance between uncorrelated states will be used below in the Fig. 5.8 (a) and Fig. 5.9. (a). 


\subsection{Stable Chaos in the Balanced State}

Similar to cLIF networks studied in Chapter 4, the dynamics of LIF networks converged to the balanced state for large recurrent inhibition. Figure 5.2 shows a representative spike pattern and voltage trace illustrating the irregular and asynchronous firing activity and strong membrane potential fluctuations. A second characteristic feature of balanced networks is a substantial heterogeneity in the spike statistics across neurons, indicated by broad distributions of coefficients of variation $(\mathrm{cv})$ and firing rates $(v)$. An important source for this heterogeneity are quenched fluctuations from the binomial-distributed indegrees in the studied random networks. Independent of model details, the network-averaged firing rate $\bar{v}$ in the balanced state can be predicted as $\bar{v}_{\text {bal }} \approx I_{0} /\left(J_{0} \tau_{\mathrm{m}}\right)$, Eq. 2.19). The good agreement of this prediction with the numerically obtained firing rate confirms the dynamical balance of excitation and inhibition in the studied networks.

Although the voltage trajectory of each neuron and the network state were very irregular, the collective dynamics of the networks was apparently completely stable (Fig. 5.3). For all firing rates, coupling strengths and connection probabilities, the whole spectrum of Lyapunov exponents (disregarding the zero exponent for perturbations tangential to the trajectory) was negative, confirming the occurrence of stable dynamics in LIF networks with respect to infinitesimal perturbations [4-7]. Our analysis furthermore shows the invariance of the Lyapunov spectra to the network size $N$ (see inset in Fig. 5.3A and more examples in Fig. 5.4. This demonstrates, to our knowledge for the first time, that this type of dynamics is extensive. With increasing connectivity $K$ all Lyapunov exponents approached a constant $\lambda_{i} \approx-1 / \tau_{\mathrm{m}}$. This is deduced from the mean Lyapunov exponent in random matrix approximation and the numerical observation that the largest exponent approached $\lambda_{\text {mean }}$ in the large $K$-limit (see Section 5.2 and Fig. 5.3B). These results suggest that in the thermodynamic limit arbitrary weak perturbations decay exponentially on the single neuron membrane time constant. As will become clear in the following, however, this issue is quite delicate.

\subsection{Firing Rate Response After Single Spike Failures}

Experimentally realizable and well-controlled state perturbations to the dynamics of cortical networks are the addition or suppression of individual spikes [101-104]. Such minimalistic neurostimulation can elicit complex behavioral responses [102-104] and can trigger a measurable rate response in intact cortical networks [101]. We therefore examined how such single spike perturbations affected the collective dynamics of our networks. Here, the simplest single spike perturbation is the suppression of a single spike. Figure 5.5 illustrates the firing rate response if one spike is skipped at $t=0$. The missing inhibition immediately triggered additional spikes in the $K$ postsynaptic neurons such that the network-averaged firing rate increased abruptly by $\delta \bar{v} \sim K \bar{v} / N$. Since the induced extra spikes inhibited further neurons in the network, the overshoot in the firing rate quickly settled back to the stationary state within a time of order $\delta t \sim 1 /(K \bar{v})$. The overall number of additional spikes in the networks therefore was $N \delta \bar{v} \delta t \approx 1$ and the one skipped spike was immediately compensated by a single extra spike. This might even be the general case if the following neuron model-independent meanfield approximation holds.

\subsubsection{Meanfield Approximation of Firing Rate Response}

The weak rate response to single spike perturbations in balanced networks can also be derived in a meanfield approximation. Without loss of generality, time will be measured in terms of the 

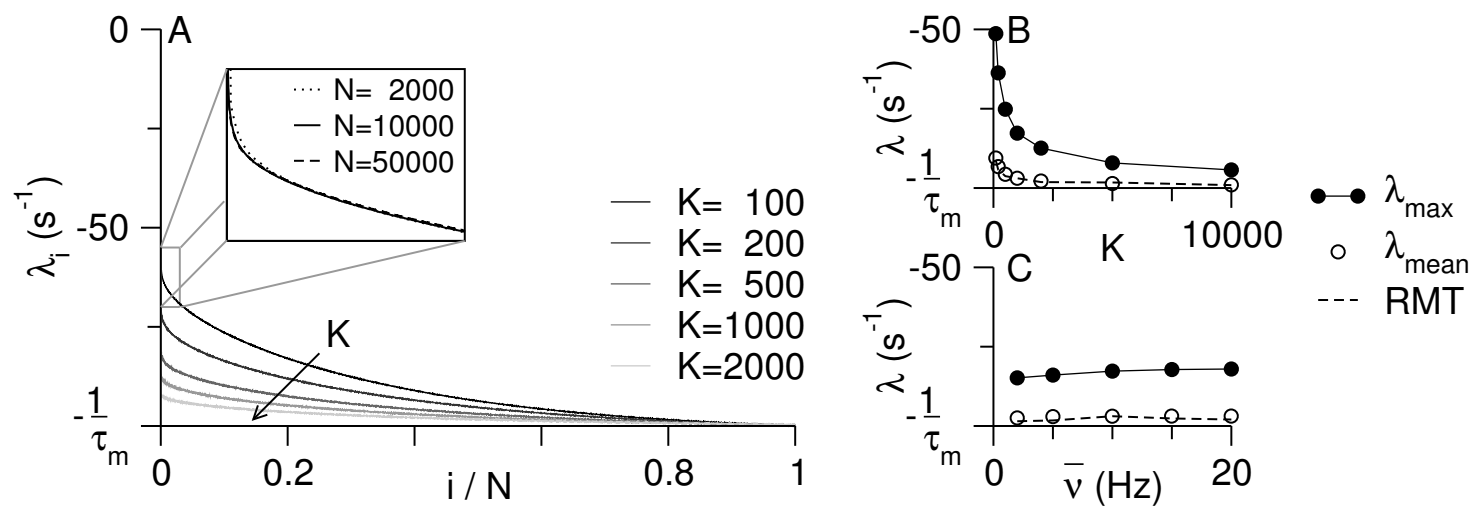

Figure 5.3 - Extensive stable dynamics with respect to infinitesimal perturbations. (A) Spectrum of Lyapunov exponents $\left\{\lambda_{i}\right\}$ of networks of $N=10000$ LIF neurons for different connectivities $K$, inset: closeup of spectra for $K=100$ and different network sizes $N$, (B,C) largest Lyapunov exponent $\lambda_{2}=\lambda_{\max }$ and mean Lyapunov exponent $\lambda_{\text {mean }}=\frac{1}{N} \sum_{i=1}^{N} \lambda_{i}$ versus connectivity $K$ and average firing rate $\bar{v}$ (dashed lines: random matrix theory for $\lambda_{\text {mean }}$, see Section 5.2), (parameters: $N=100000, K=1000, \bar{v}=10 \mathrm{~Hz}, J_{0}=1$, $\tau_{\mathrm{m}}=10 \mathrm{~ms}$; averages of 10 initial conditions).

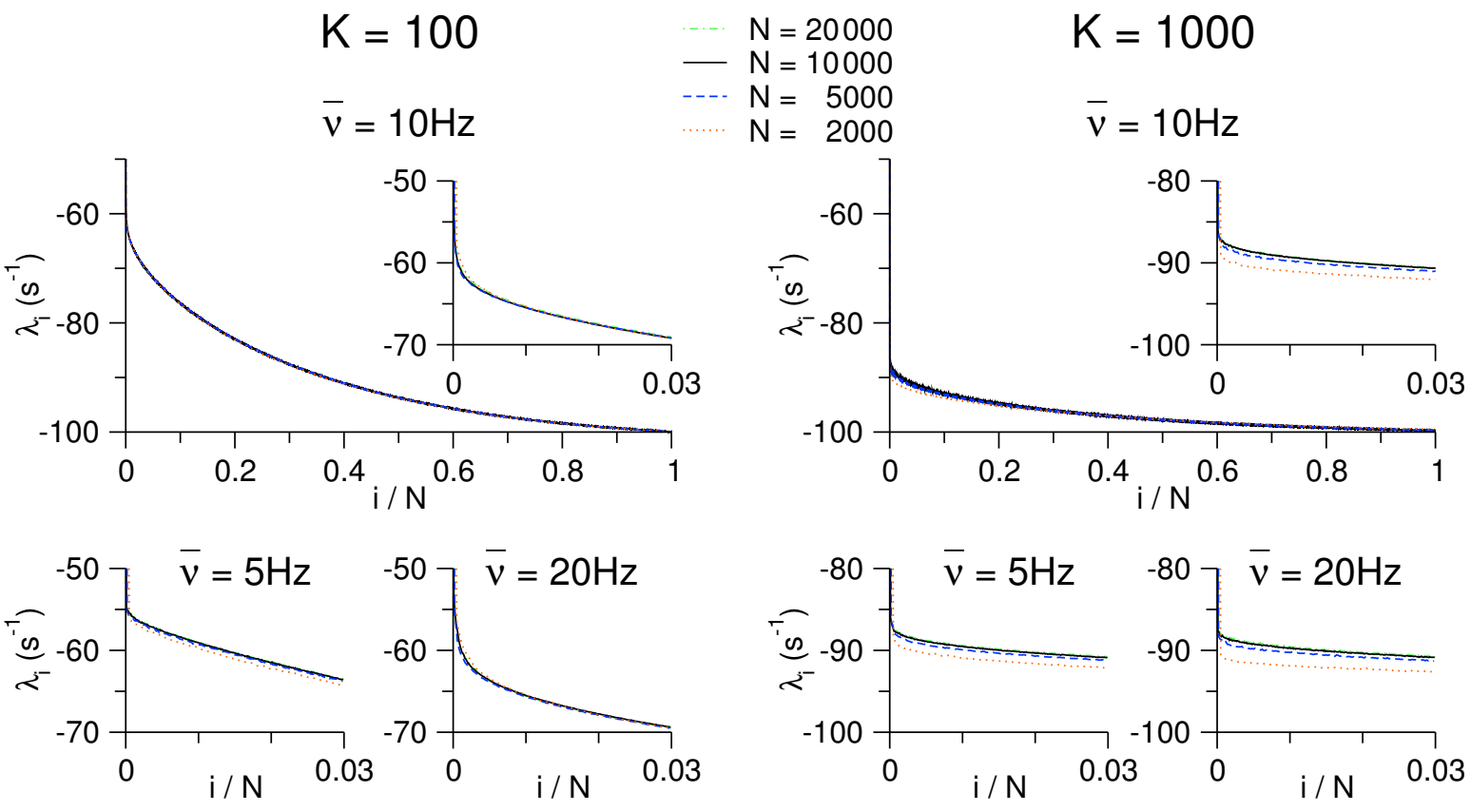

Figure 5.4 - Invariance of Lyapunov spectra for large network sizes $N$. Left panels: connectivity $K=100$ and different network-averaged firing rates $\bar{v}$, right panels: connectivity $K=1000$ and different network-averaged firing rates $\bar{v}$, (other parameters: $J_{0}=1, \tau_{\mathrm{m}}=10 \mathrm{~ms}$ ). 

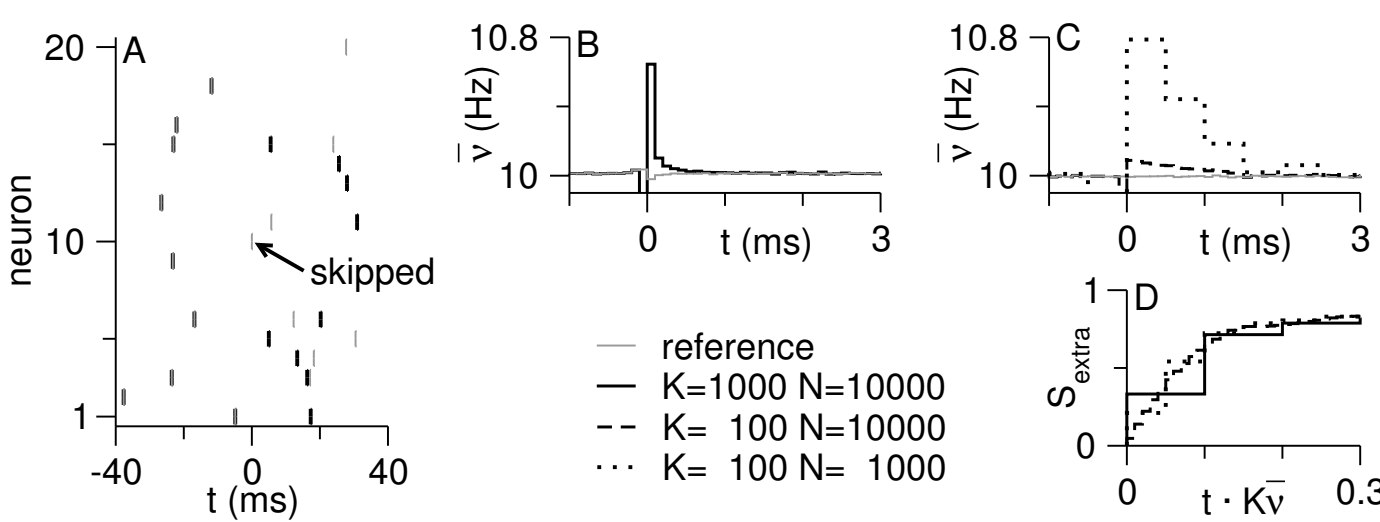

- reference

- $K=1000 \mathrm{~N}=10000$

$-\mathrm{K}=100 \mathrm{~N}=10000$

$\cdots K=100 \mathrm{~N}=1000$

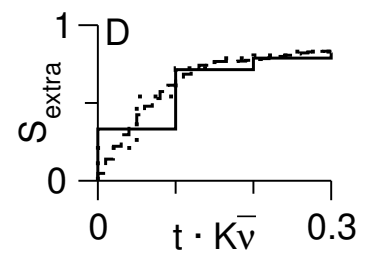

Figure 5.5 - Weak firing rate response after single spike failure. (A) Sample spike pattern of 20 randomly chosen neurons (gray: reference trajectory, black: single spike skipped at $t=0$ ), (B) network-averaged firing rate of reference trajectory $\bar{v}$ and in response to skipped spike $\tilde{v}$ versus time for different connectivities $K$ and network sizes $N,(\mathrm{C})$ number of extra spikes $S_{\text {extra }}=N \int(\tilde{v}-\bar{v}) \mathrm{d} t$ in the entire network versus time (rescaled with average input rate $K \bar{v}$ ), (parameters: $\bar{v}=10 \mathrm{~Hz}, J_{0}=1, \tau_{\mathrm{m}}=10 \mathrm{~ms}$; averages of 100 initial conditions with 10000 calculations each).

membrane time constant for simplicity in this derivation $t \mapsto t / \tau_{\mathrm{m}}$. Two coding channels contribute to the rate response $\delta v(t)$, one due to the change in the mean input current $\mu(t)$ and one due to the change in the variance of the input current $\sigma(t)$.

The mean input current $\mu=\sqrt{K}\left(I_{0}-J_{0} \bar{v}\right)$ changes in the wake of a single spike failure by

$$
\delta \mu=\frac{\sqrt{K}}{N} J_{0} \delta(t)-\sqrt{K} J_{0} \delta v(t) .
$$

The first term reflects the instantaneous effect of the one missed spike at $t=0$, equivalent to a positive current pulse of strength $J_{0} / \sqrt{K}$ in $K$ postsynaptic neurons averaged over the entire population. The second term reflects the change in the recurrent inhibition due to the change in the average firing rate. The average additional and missed spikes captured in the rate response $\delta v(t)$ each lead to $K$ current pulses of strength $-J_{0} / \sqrt{K}$.

Additionally, the change in the average firing rate $\delta v(t)$ leads to the following magnitude of input current fluctuations $\tilde{\sigma}(t)=J_{0} \sqrt{\bar{v}+\delta v(t)}$. The differences of the fluctuations to the stationary state $\sigma(t)=J_{0} \sqrt{\bar{v}}$ is thus

$$
\begin{array}{ccc}
\delta \sigma & = & J_{0} \sqrt{\bar{v}+\delta v(t)}-J_{0} \sqrt{\bar{v}} \\
& \stackrel{(t) \rightarrow 0}{\simeq} & J_{0} \frac{1}{2 \sqrt{\bar{v}}} \delta v(t)+\mathscr{O}\left(\delta v(t)^{2}\right) .
\end{array}
$$

Here we have assumed a weak rate response. This needs to be validated at the end.

The rate response is given by the convolutions of (5.21) and (5.22) with the response functions in linear response theory for the mean $v_{1}^{\mu}(t)$ and variance $v_{1}^{\sigma}(t)$ :

$$
\delta \bar{v}(t)=v_{1}^{\mu}(t) \star \delta \mu(t)+v_{1}^{\sigma}(t) \star \delta \sigma(t)
$$

These might be derived for the specific neuron model but in fact we will use self-consistency arguments without the explicit expressions for $v_{1}^{\mu}(t)$ and $v_{1}^{\sigma}(t)$. After a Fourier transformation of 
(5.23), the convolutions become products such that

$$
\begin{aligned}
\delta \tilde{v}(\omega) & =\tilde{v}_{1}^{\mu}(\omega) \delta \mu(\omega)+\tilde{v}_{1}^{\sigma}(\omega) \delta \sigma(\omega) \\
& =\tilde{v}_{1}^{\mu}(\omega)\left(\frac{\sqrt{K}}{N} J_{0}-\sqrt{K} J_{0} \delta \tilde{v}(\omega)\right)+\tilde{v}_{1}^{\sigma}(\omega) \frac{J_{0}}{2 \sqrt{\bar{v}}} \delta \tilde{v}(\omega)
\end{aligned}
$$

Solving this for $\delta \tilde{v}(\omega)$ yields

$$
\begin{aligned}
\delta \tilde{v}(\omega) & =\frac{1}{N} \frac{\sqrt{K} J_{0} \tilde{v}_{1}^{\mu}(\omega)}{1+\sqrt{K} J_{0} \tilde{v}_{1}^{\mu}(\omega)-\frac{J_{0}}{2 \sqrt{\bar{v}}} \tilde{v}_{1}^{\sigma}(\omega)} \\
& =\frac{1}{N} \frac{\tilde{v}_{1}^{\mu}(\omega)}{\tilde{v}_{1}^{\mu}(\omega)+\frac{1}{\sqrt{K} J_{0}}-\frac{1}{2 \sqrt{K \bar{v}}} \tilde{v}_{1}^{\sigma}(\omega)} .
\end{aligned}
$$

As expected, the single spike rate response vanishes in the large $N$ limit. For large $K$ (with $1 \ll K \ll N)$, the rate response becomes

$$
\begin{aligned}
\delta \tilde{v}(\omega) & \simeq \frac{1}{N}\left(1-\left(\frac{1}{J_{0} \tilde{v}_{1}^{\mu}(\omega)}-\frac{1}{2 \sqrt{\bar{v}}} \frac{\tilde{v}_{1}^{\sigma}(\omega)}{\tilde{v}_{1}^{\mu}(\omega)}\right) \frac{1}{\sqrt{K}}+\mathscr{O}\left(\frac{1}{K}\right)\right) \\
\delta v(t) & \simeq \frac{1}{N}\left(\delta(t)+\mathscr{O}\left(\frac{1}{\sqrt{K}}\right)\right) .
\end{aligned}
$$

The number of extra spikes in networks with high connectivity is therefore

$$
S_{\text {extra }}=N \int \delta v(t) \mathrm{d} t=1
$$

Hence, the one missed spike is just compensated by one extra spike in the balanced network confirming the numerically obtained result displayed in Fig. 5.5 .

\subsection{Exponential State Separation After Single Spike Failures}

Even though the failure of one individual spike resulted in very weak and brief firing rate responses, it nevertheless induced rapid state decoherence. We analyzed the distance $D_{\phi}(t)=$ $\frac{1}{N} \sum_{i}\left|\tilde{\phi}_{i}(t)-\phi_{i}(t)\right|$ between the perturbed trajectory with an individual spike failure at $t=0$ and the reference trajectory. After a single spike failure, all trajectories separated exponentially fast from the reference trajectory at a surprisingly high rate. The averages of several calculations with different initial conditions are summarized in Fig. 5.6. Because this exponential separation of nearby trajectories is reminiscent of deterministic chaos, we call its separation rate the pseudo Lyapunov exponent $\lambda_{p}$. The pseudo Lyapunov exponent was network size invariant, but showed a completely different behavior compared to the classical Lyapunov exponents. With increasing connectivity, it appears to diverge linearly $\lambda_{p} \sim K \bar{v}$. It is thus expected to grow to infinity in the high connectivity limit, reminiscent of binary neuron networks exhibiting an infinite Lyapunov exponent in the thermodynamic limit [2, 3]. 

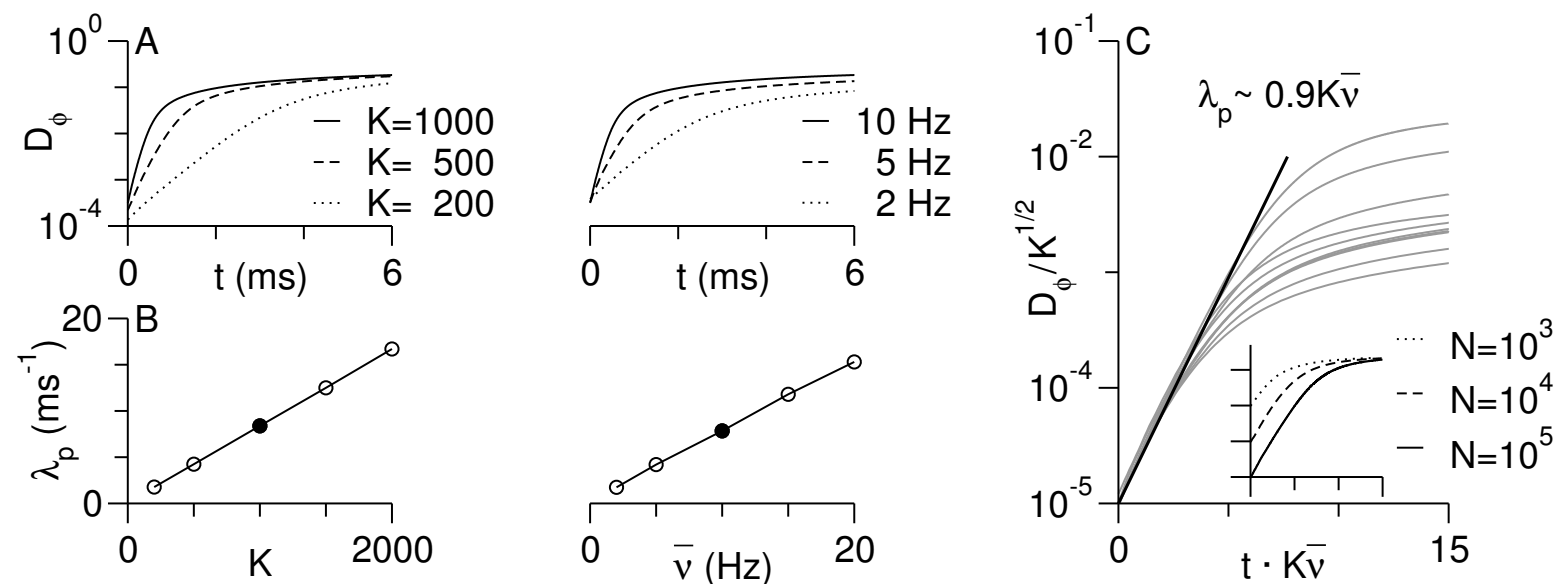

Figure 5.6 - Sensitivity to single spike failures. (A) Average distance $D_{\phi}$ between trajectory after spike failure and reference trajectory versus time in log-lin plots for different connectivities $K$ and average firing rates $\bar{v}$, (B) pseudo Lyapunov exponent $\lambda_{p}$ from exponential fits $D_{\phi} \sim \exp \left(\lambda_{p} t\right)$ before reaching saturation versus connectivity $K$ and average firing rate $\bar{v}$, (C) distance-evolution of all parameter sets (rescaled with approximate perturbation strength $K J_{0} / \sqrt{K}$ ) versus time (rescaled with average input rate $K \bar{v}$ ) collapse to characteristic exponential state separation with rate $\lambda_{p} \sim 0.9 K \bar{v}$ (inset: different network sizes $N$ for $K=100$ ), (parameters: $N=100000, K=1000, \bar{v}=10 \mathrm{~Hz}, J_{0}=1, \tau_{\mathrm{m}}=10 \mathrm{~ms}$; averages of 10 initial conditions with 100 calculations each).

\subsection{Exponentially Separating Flux Tubes Around Stable Trajectories}

In the same balanced LIF networks, we thus find stable dynamics in response to infinitesimal perturbations and unstable dynamics in response to single spike failures. To further analyze the transition between these completely opposite behaviors, we applied finite perturbations of variable size perpendicular to the state trajectory (Fig. 5.7). Depending on the perturbation strength $\varepsilon$ and direction $\delta \vec{\phi}$ (with $\sum_{i} \delta \phi_{i}^{2}=1$ ), the perturbed trajectory either converged back to the reference trajectory or diverged exponentially fast. The probability $P_{S}(\varepsilon)$ that a perturbation of strength $\varepsilon$ induced exponential state separation was very well fitted by $P_{S}(\varepsilon)=1-\exp \left(-\varepsilon / \varepsilon_{\mathrm{ft}}\right)$. Hence, $\varepsilon_{\mathrm{ft}}$ is a characteristic phase space distance separating stable from unstable dynamics. Intriguingly, this distance decreased as $\varepsilon_{\mathrm{ft}} \sim N^{-0.5} K^{-0.4} \bar{v}^{-1}$ (Fig. 5.8). For large $K$ and $N$ the dynamics in the thermodynamic limit $(N \rightarrow \infty)$ would thus be unstable even to infinitesimal perturbations $(\varepsilon \rightarrow 0)$. Contrary, the analysis of the Lyapunov spectra has shown that taking the limit $\varepsilon \rightarrow 0$ first and then $N \rightarrow \infty$ yields stable dynamics. Thus, the order of the limits appears crucial in defining the dynamical nature of balanced LIF networks in the thermodynamic limit.

The evolution of finite perturbations suggests an exotic phase space structure of stable flux tubes around unique trajectories (Fig. 5.7(d)). Perturbations within these flux tubes decayed exponentially, whereas perturbations greater than the typical flux tube radius $\varepsilon_{\mathrm{ft}}$ induced exponential state separation. Single synaptic failures correspond to small perturbations of size $\varepsilon_{\text {syn }} \approx J_{0} / \sqrt{K N}$ and therefore had an $N$ indpendent and almost $K$ independent probability of inducing exponential state separation (Fig. 5.9). This probability increased linearly with the average firing rate $\bar{v}$. 


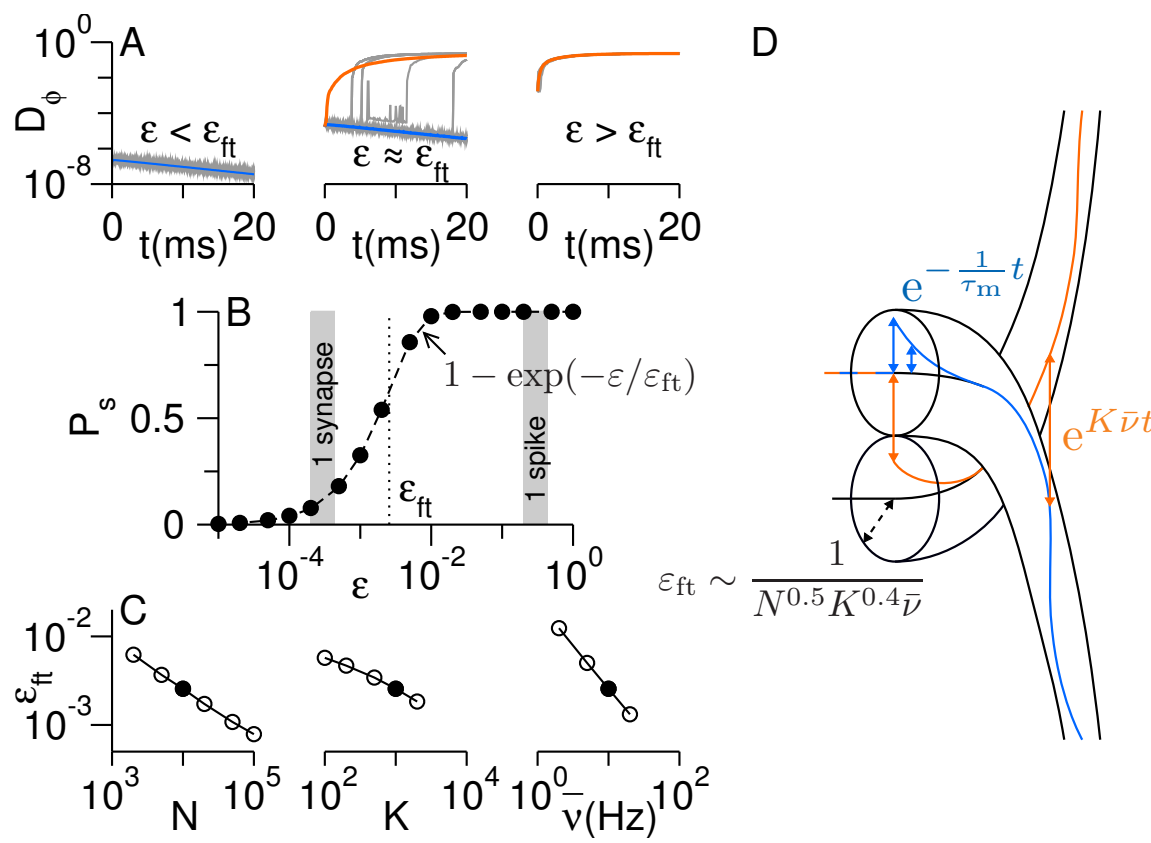

Figure 5.7 - Sensitivity to finite-size perturbations. (A) Distance $D_{\phi}$ between perturbed and reference trajectory measured at spike times of reference trajectory (projecting out possible time-shifts) for perturbations of strengths $\varepsilon=0.00002,0.002,0.2$ in log-lin plots (gray lines: 20 examples for initial perturbations of same size pointing in different random directions perpendicular to trajectory, color lines: averages of exponentially separating/converging cases), (B) probability $P_{s}$ of exponential state separation versus perturbation strength $\varepsilon$ in lin-log plot (dashed line: fit to $P_{s}(\varepsilon)=1-\exp \left(-\varepsilon / \varepsilon_{\mathrm{ft}}\right)$, dotted line: characteristic perturbation size $\varepsilon_{\mathrm{ft}}$ separating stable from unstable dynamics, shaded areas: strengths corresponding to single synapse and single spike failures), (C) characteristic perturbation size $\varepsilon_{\mathrm{ft}}$ versus network size $N$, connectivity $K$ and average firing rate $\bar{v}$ in log-log plots, (D) symbolic picture of stable flux tubes with radius $\varepsilon_{\mathrm{ft}}$ (stable dynamics inside flux tube but exponential separation of adjacent flux tubes), (parameters: $N=10000, K=1000$, $\bar{v}=10 \mathrm{~Hz}, J_{0}=1, \tau_{\mathrm{m}}=10 \mathrm{~ms}$; averages of 10 initial conditions with 100 calculations and 100 random directions each).

\subsection{Single Spike Perturbation in Rapid Theta Neuron Networks}

Because the LIF model is a special case of single neuron dynamics with instantaneous AP initiation, we wanted to compare the results of the networks dynamics with respect to single spike failures with networks of neurons with finite AP onset rapidness. We therefore repeated the calculations of the distance between reference trajectories and trajectories after single spike failures in rapid theta neuron networks with variable AP onset rapidness (Fig. 5.10, 5.12).

We start with repeating the results of LIF networks completeness. These networks with instant AP generation, comparable with $r \rightarrow \infty$ in the rapid theta neuron model, exhibit a scaling of the pseudo Lyapunov exponent with the connectivity $K$ and average firing rate $\bar{v}$ as $\lambda_{p} \sim K^{0.95} \bar{v}^{0.95}$ (Fig. 5.10(a)). The pseudo Lyapunov exponent thus seems to grow to infinity in the large connectivity limit.

Networks of rapid theta neurons and an extremely high AP onset rapidness $r=1000$ exhibit a similar scaling law as LIF networks. The pseudo Lyapunov exponent seems to scale as $\lambda_{p} \sim$ $K^{0.85} \bar{v}^{0.93}$ (Fig. 5.10(b)) and would thus tend to infinity in the high connectivity limit. Decreasing 

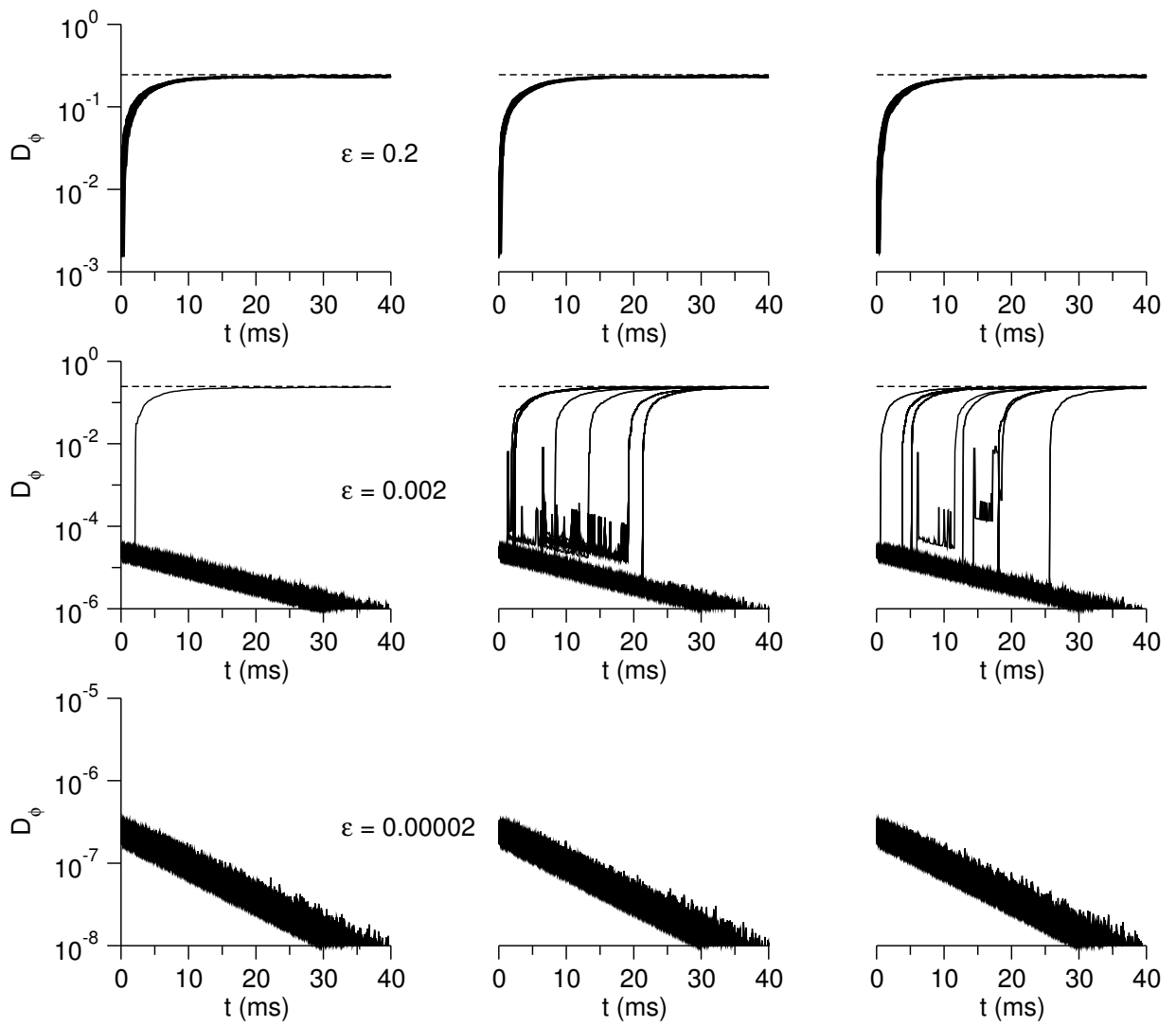

(a) Finite size perturbations, examples of distance evolution at 3 different states for 3 perturbation strengths $\varepsilon$ pointing in 100 random directions. Dashed lines: mean-field approximation of distance between uncorrelated states, Eq. (5.20).
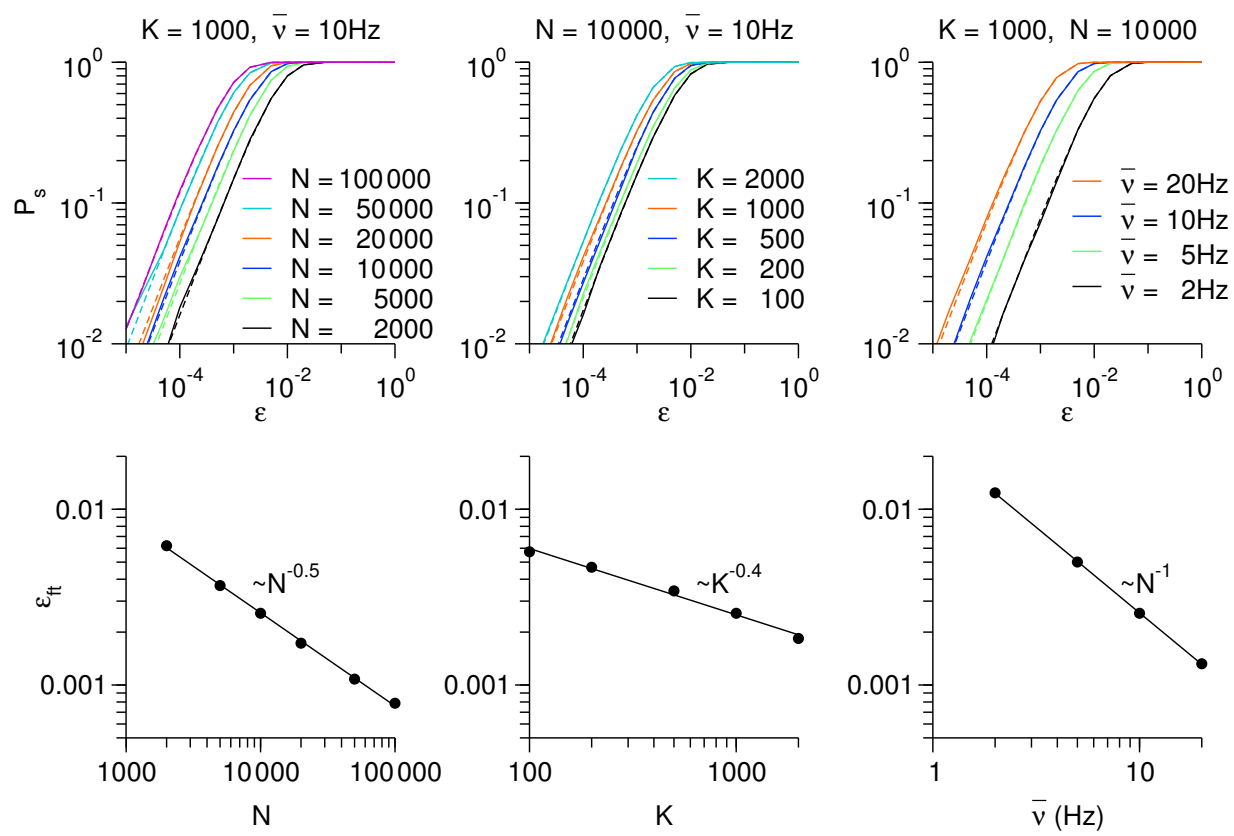

Figure 5.8 - Flux tube radius estimation for different network sizes $N$, connectivities $K$ and networkaveraged firing rates $\bar{v}$. Top panels: Probability $P_{\mathrm{S}}$ (straight lines) that the perturbation of strength $\varepsilon$ induced a switch to a different exponentially separating flux-tube and fits to $P_{s}(\varepsilon)=1-\exp \left(-\varepsilon / \varepsilon_{\mathrm{ft}}\right.$ ) (dashed lines), bottom panels: Flux-tube radius $\varepsilon_{\mathrm{ft}}$ versus network sizes $N$, connectivities $K$ and network-averaged firing rates $\bar{v}$ (straight lines from power law fits), (parameters: $N=10000, K=1000, \bar{v}=10 \mathrm{~Hz}, J_{0}=1$, $\tau_{\mathrm{m}}=10 \mathrm{~ms}$; averages of 10 initial conditions with 100 calculations and 100 random directions each) 

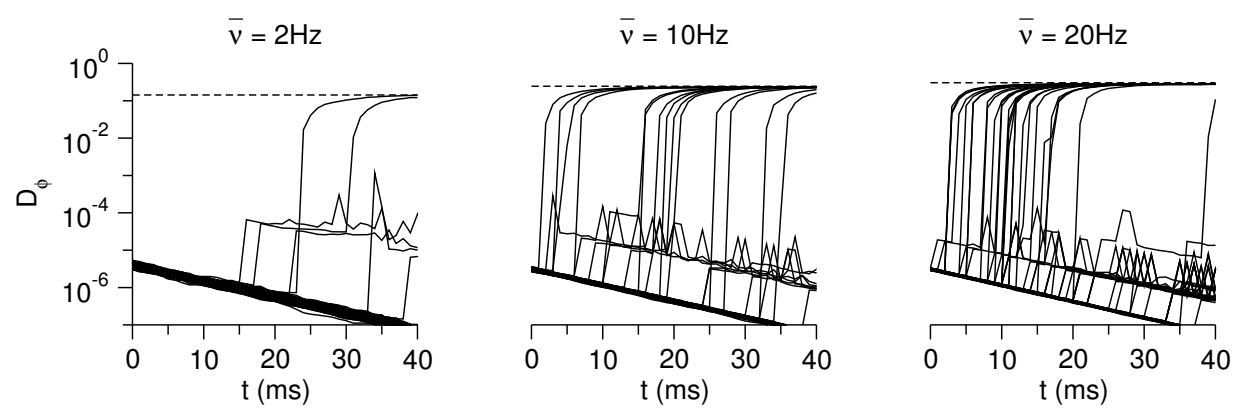

(a) Examples of distance evolution at 100 different states after single synapse failure at $t=0$ for 3 network-averaged firing rates $\bar{v}$. Dashed lines: mean-field approximation of distance between uncorrelated states, Eq. 5.20).
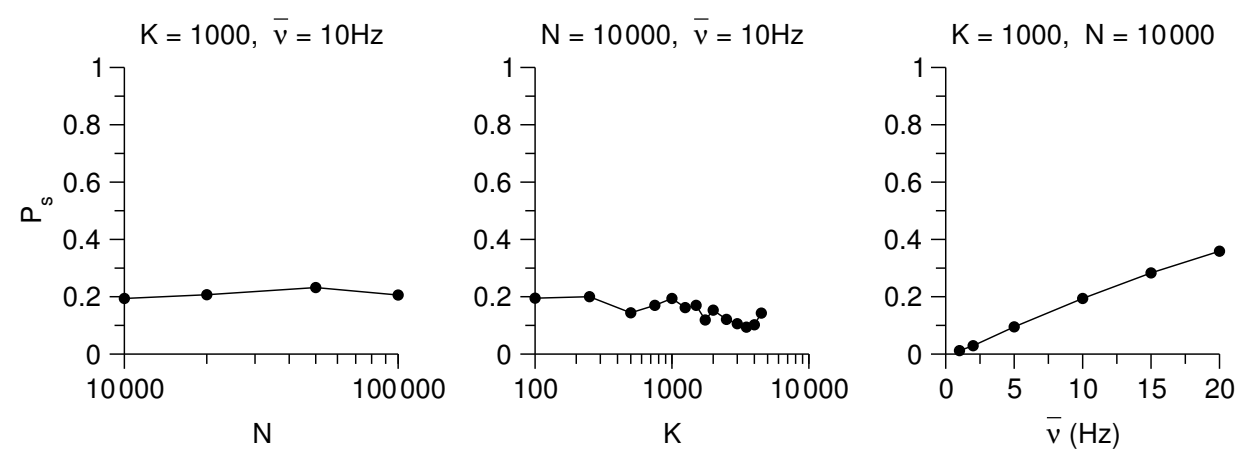

Figure 5.9 - Probability $P_{\mathrm{s}}$ for single synaptic failures to induce switch to a different exponentially separating flux tube for different network sizes $N$, connectivities $K$ and network-averaged firing rates $\bar{v}$, (parameters: $N=10000, K=1000, \bar{v}=10 \mathrm{~Hz}, J_{0}=1, \tau_{\mathrm{m}}=10 \mathrm{~ms}$; averages of 10 initial conditions with 100 calculations each)

the AP onset rapidness to $r=100$ leads to a similar scaling $\lambda_{p} \sim K^{0.85} \bar{v}^{0.92}$ (Fig. 5.11(a)).

Further decreasing the AP onset rapidness to $r=10$ breaks this trend and it is clearly visible that the pseudo Lyapunov exponent converges with $K$ (Fig. 5.11(b)). This is also the case for low AP onset rapidness $r=3$ and $r=1$ (Fig. 5.12). We can thus conclude that for low AP onset rapidness, the pseudo Lyapunov exponent with respect to finite perturbations converges to the standard Lyapunov exponent with respect to infinitesimal perturbations.

We have seen a gradual change between the results of LIF networks $(r \rightarrow \infty)$ towards the results of theta networks $(r=1)$ with decreasing AP onset rapidness $r$. This suggests that the exotic phase space structure of stable flux tubes around unique stable trajectories is a result of instantaneous AP initiation and demonstrates how crucial the AP generation of single neurons is in determining the collective network dynamics. 

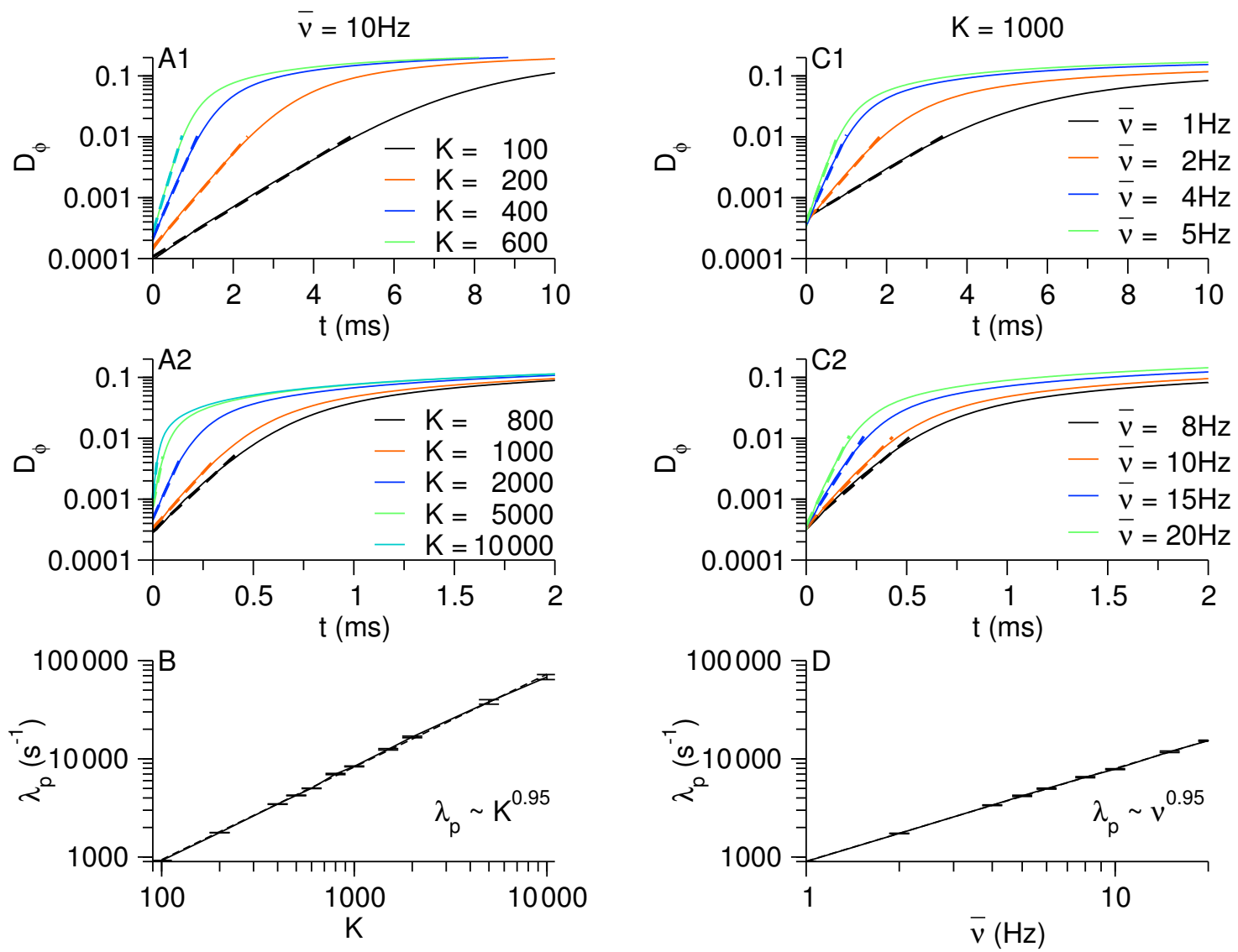

(a) LIF, comparable with $r \rightarrow \infty(K=1000, \bar{v}=10 \mathrm{~Hz})$
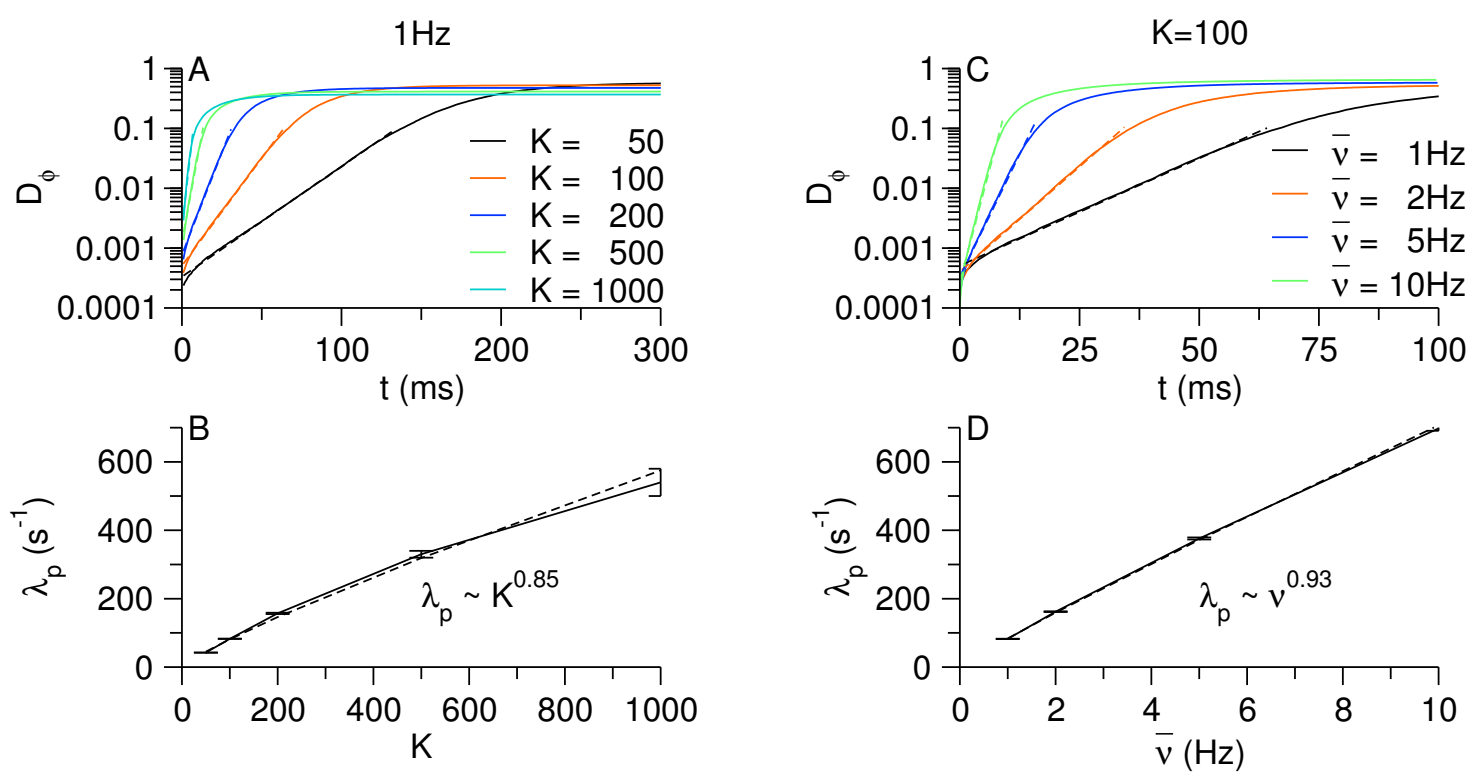

(b) $r=1000(K=100, \bar{v}=1 \mathrm{~Hz})$

Figure 5.10 - Sensitivity to single spike failures in networks with (almost) instantaneous AP generation. (A,C) Average distance $D_{\phi}$ between trajectory after spike failure and reference trajectory versus time in log-lin plots for different connectivities $K$ and average firing rates $\bar{v}$, (B,D) pseudo Lyapunov exponent $\lambda_{p}$ from exponential fits $D_{\phi} \sim \exp \left(\lambda_{p} t\right)$ before reaching saturation versus connectivity $K$ and average firing rate $\bar{v}$, (parameters: $N=100000, J_{0}=1, \tau_{\mathrm{m}}=10 \mathrm{~ms}$; averages of 10 initial conditions with 100 calculations each). 

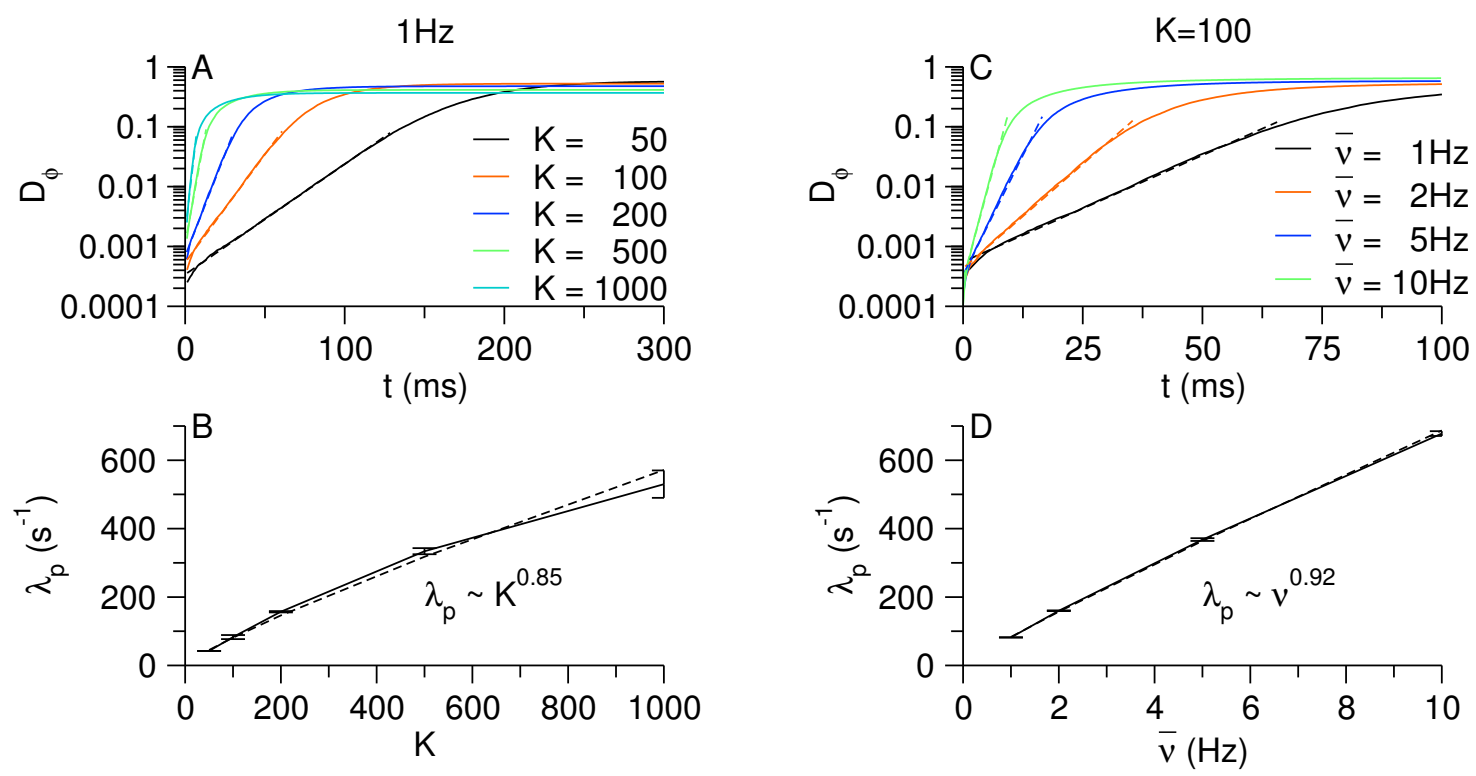

(a) $r=100$
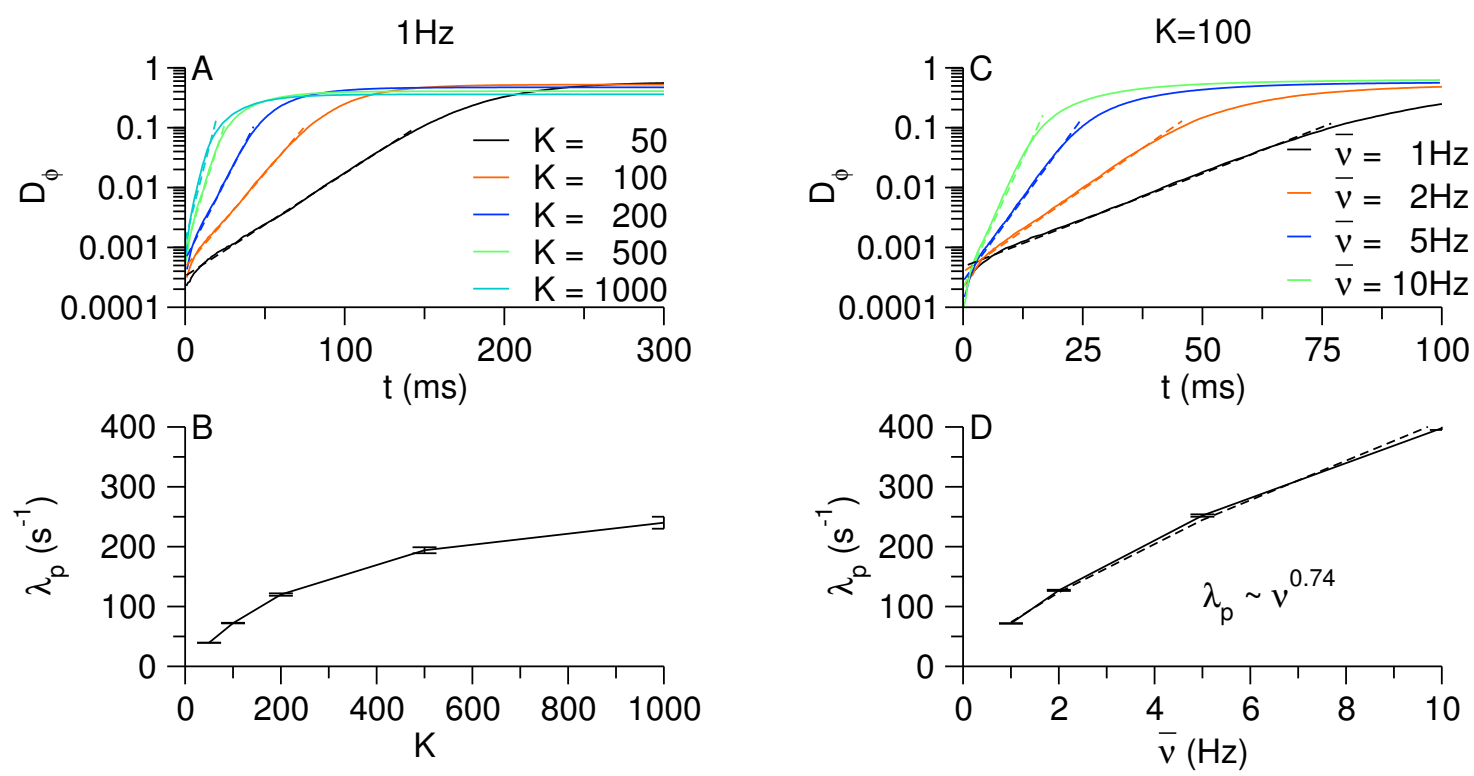

(b) $r=10$

Figure 5.11 - Sensitivity to single spike failures in networks with high AP onset rapidness. (A,C) Average distance $D_{\phi}$ between trajectory after spike failure and reference trajectory versus time in log-lin plots for different connectivities $K$ and average firing rates $\bar{v}$, (B,D) pseudo Lyapunov exponent $\lambda_{p}$ from exponential fits $D_{\phi} \sim \exp \left(\lambda_{p} t\right)$ before reaching saturation versus connectivity $K$ and average firing rate $\bar{v}$, (parameters: $N=100000, K=100, \bar{v}=1 \mathrm{~Hz}, J_{0}=1, \tau_{\mathrm{m}}=10 \mathrm{~ms}$; averages of 10 initial conditions with 100 calculations each). 

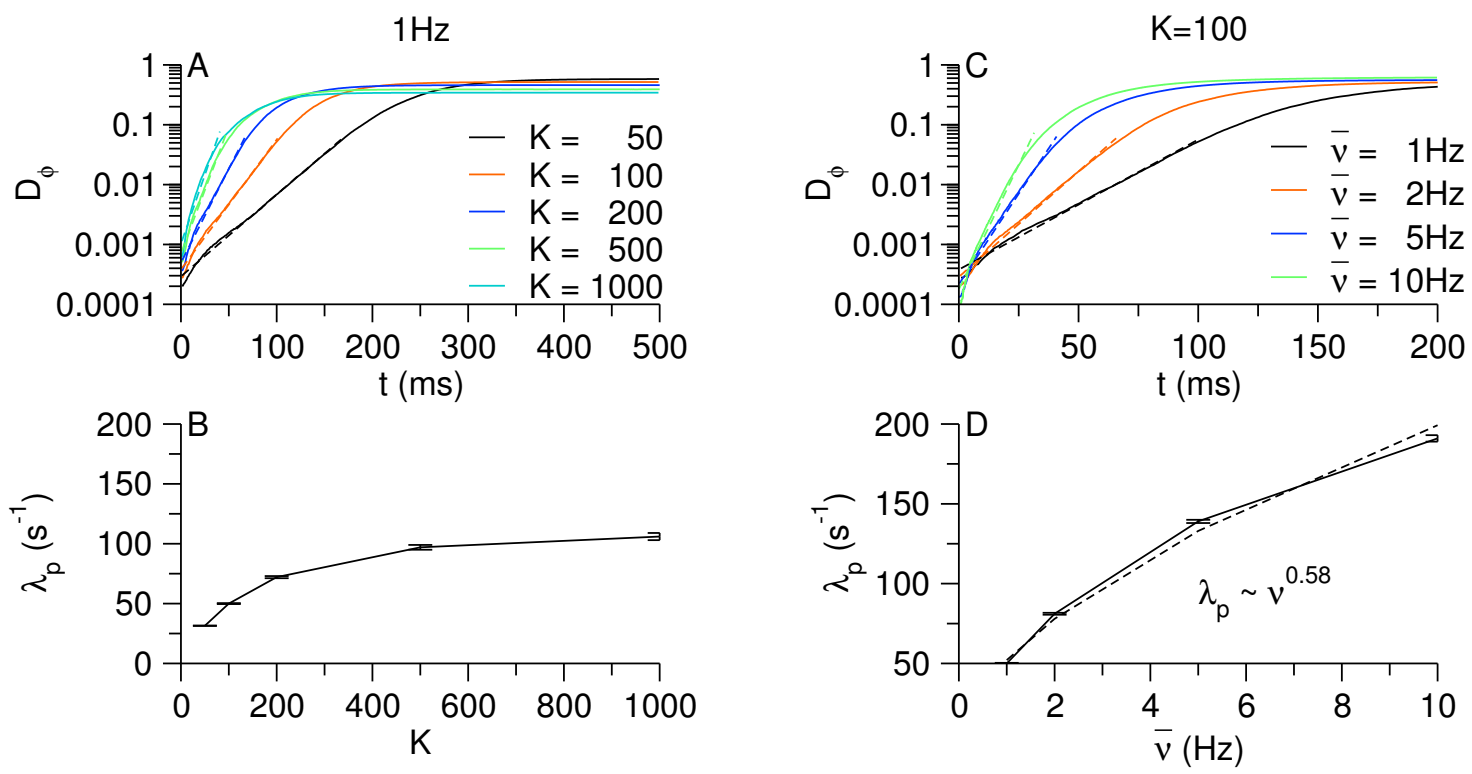

(a) $r=3$
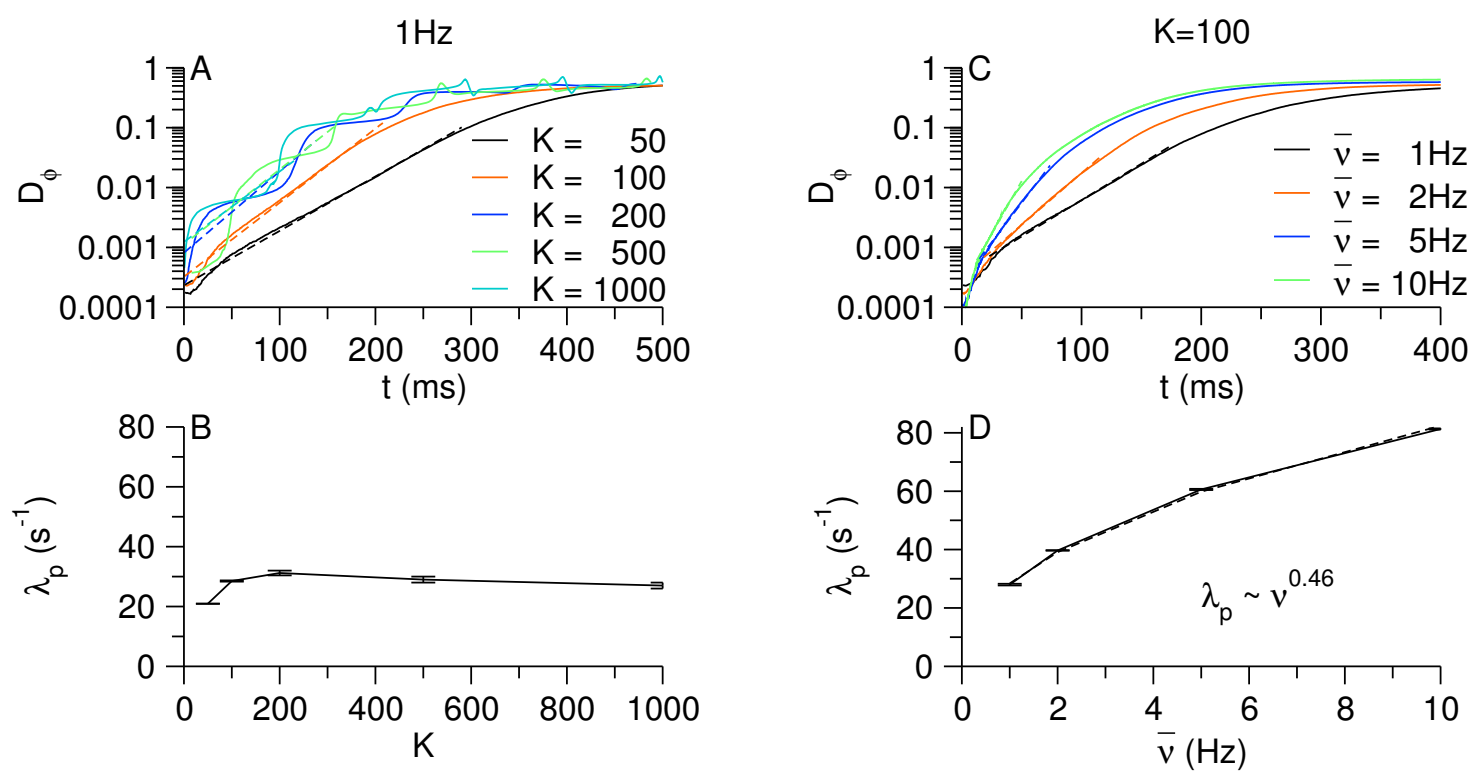

(b) $r=1$

Figure 5.12 - Sensitivity to single spike failures in networks with low AP onset rapidness. (A,C) Average distance $D_{\phi}$ between trajectory after spike failure and reference trajectory versus time in log-lin plots for different connectivities $K$ and average firing rates $\bar{v}$, (B,D) pseudo Lyapunov exponent $\lambda_{p}$ from exponential fits $D_{\phi} \sim \exp \left(\lambda_{p} t\right)$ before reaching saturation versus connectivity $K$ and average firing rate $\bar{v}$, (parameters: $N=100000, K=100, \bar{v}=1 \mathrm{~Hz}, J_{0}=1, \tau_{\mathrm{m}}=10 \mathrm{~ms}$; averages of 10 initial conditions with 100 calculations each). 


\subsection{Summary}

Motivated by the recent observation that real cortical networks are highly sensitive to single spike perturbations [101], we examined in this chapter how single spike perturbations evolve in the formally stable dynamics of inhibitory LIF networks. Our analysis revealed the cooccurence of dynamical stability to infinitesimal state perturbations and sensitive dependence on single spike and even single synapse perturbations in the dynamics of such networks.

Networks of inhibitory LIF neurons exhibit a negative definite extensive Lyapunov spectrum that at first sight suggests a well-defined thermodynamic limit of the network dynamics characterized by stable chaos as previously proposed [4-7]. In this dynamics, single spike failures induce extremely weak firing rate responses that become basically negligible for large networks. Nevertheless, such single spike perturbations typically put the network state on a very different dynamical path that diverges exponentially from the original one. The rate of exponential state separation was quantified with the so-called pseudo Lyapunov exponent $\lambda_{p}$. The scaling of $\lambda_{p} \sim K \bar{v}$ implies extremely rapid, practically instantaneous, decorrelation of network microstates, which is reminiscent of the results obtained in binary neuron networks in the balanced state [2,3].

Our results suggest that the seemingly paradoxical coexistence of local stability and exponential state separation reflects the partitioning of the networks' phase space into a tangle of flux tubes. States within a flux tube are attracted to a unique, dynamically stable trajectory. Different flux tubes, however, separate exponentially fast. The decreasing flux tube radius in the large system limit suggests that an unstable dynamics dominates the thermodynamic limit. The resulting sensitivity to initial conditions is described by the rate of flux tube separation, the pseudo Lyapunov exponent, that showed no sign of saturation. These findings suggest that the previously reported infinite Lyapunov exponent on the one hand [2,3] and local stability on the other hand [4-7] resulted from the order in which the weak perturbation limit and the thermodynamic limit were taken.

For finite networks, the phase space structure revealed here may provide a basis for insensitivity to small perturbations (e.g. noise or variations in external inputs) and strong sensitivity to larger perturbations. In the context of reservoir computing, the flux tube radius defines a border between the fading property (variations of initial conditions smaller $\varepsilon_{\mathrm{ft}}$ die out exponentially) and the separation property (input variations larger $\varepsilon_{\mathrm{ft}}$ cause exponentially separating trajectories). Applications of LIF neuron networks in reservoir computing may thus strongly benefit if the flux tube structure of the network phase space is taken into account. Our results of a very high pseudo Lyapunov exponent also reveal that the notion of an 'edge of chaos' is not applicable in these leaky integrate and fire networks.

The investigation of the pseudo Lyapunov exponent in rapid theta neuron networks suggested a convergence of the scaling of the pseudo Lyapunov exponent to that of LIF networks in the limit of instantaneous AP onset rapidness. This indicates that these results are not just an artifact of the instantaneous AP initiation in the LIF model but might be relevant in real cortical networks with finite but very large AP onset rapidness. 


\section{Discussion}

In this thesis, we introduced a novel approach to precisely characterize and quantify the collective dynamics of spiking neuron networks. Based on numerically exact event-based simulations and the calculation of the complete spectrum of Lyapunov exponents of neural networks, it allows for the clear differentiation between stable and chaotic dynamics and provides a measure of the dynamical entropy production in chaotic neural networks. This approach can be directly applied to a wide range of $\delta$ pulse coupled neural networks with arbitrary network topology, heterogeneous single neuron parameters and single neuron phase-response curves. Here, we investigated the dynamics of large neural networks of different integrate and fire neurons in the balanced state with special focus on the influence of the single neuron action potential (AP) generation on the collective network dynamics. The balanced state is the prevailing model for the emergence of asynchronous irregular activity as observed in cortical networks.

The presented results indicate that a chaotic dynamics in neural networks in the balanced state is rather the standard than the exception. This is less surprising from a nonlinear dynamics perspective since chaos could be expected in such high-dimensional deterministic nonlinear systems. From a biological perspective this is more of a surprise, as chaos in neural networks seems at first sight quite counterintuitive to the sophisticated performance of the brain. Whether such models of neural networks as studied here exhibit chaotic or stable dynamics is therefore an important question, the answer to which yields profound insight for the understanding of the functioning of the brain.

Previous efforts to characterize the dynamics of neural networks in the balanced state have led to controversial results. Originally, it was discovered that networks of binary neurons exhibit an extreme form of chaos characterized by an infinite Lyapunov exponent [2,3]. More recently, it was shown that networks of leaky integrate and fire neurons display so-called stable chaos characterized by negative Lyapunov exponents [4-7]. Here, we have examined in detail how the dynamics of neural networks in the balanced state depends on the specific properties of the single neurons dynamics and have found AP initiation to play a fundamental role in the collective network dynamics.

As opposed to binary neurons and leaky integrate and fire neurons used in previous studies, the theta neuron model used here incorporates a dynamic AP generation comparable to other standard conductance-based neuron models. It is furthermore the canonical form of type I excitable neurons. We therefore believe that our results are representative of a wide class of neural networks. A comprehensive analysis of the dynamics of theta neuron networks in the balanced state with the here-developed approach demonstrates that such networks composed of exclusively inhibitory or excitatory and inhibitory theta neurons both exhibit extensive deterministic chaos (Chapter 2). This conventional form of chaos is characterized by positive and finite Lyapunov exponents, fat chaotic attractors and rapid dynamical entropy production. Upon activation of the excitatory-inhibitory feedback loops, the chaos intensifies, but in principle, the dynamics of excitatory-inhibitory net- 
works is already very well captured by inhibitory theta neuron networks in which the recurrent inhibition balances excitatory external currents. Our results indicate that the instability incorporated in the theta neuron model that is responsible for the AP initiation renders the collective dynamics of balanced networks robustly chaotic.

These findings, along with the recent observation that cortical neurons exhibit a much sharper AP initiation than expected from standard neuron models [1], motivated a thorough investigation of the influence of the AP onset rapidness of the single neurons on the collective network dynamics. In order to apply the here-presented approach, we developed a new exactly-solvable neuron model with variable AP onset rapidness called the rapid theta neuron model. It is based on the theta neuron model but allows for an arbitrary choice of the AP onset rapidness. This includes the limit of instantaneous AP initiation and thus also bridges the gap between the theta neuron model and the leaky integrate and fire neuron model.

Applying the approach introduced in Chapter 2 to rapid theta neuron networks revealed a strong effect of the AP onset rapidness of the single neurons. The network dynamics qualitatively change upon increasing the AP onset rapidness of the single neurons (Chapter 3). Networks of neurons with low AP onset rapidness up to about $r=10$ exhibit dynamics similar to theta neuron networks characterized by strong extensive chaos. Networks of neurons with larger AP onset rapidness also exhibit extensive chaos, but with increasing AP onset rapidness the strength of chaos strictly decreases. This is quantified by a monotonously decreasing largest Lyapunov exponent, attractor dimension and entropy production rate. A very large AP onset rapidness led to a transition to stable dynamics at the so-called edge of chaos. Neural networks at the edge of chaos are expected to be optimal for complex computations [105]. The critical AP onset rapidness in rapid theta neuron networks defining the edge of chaos varied with the network parameters. Nevertheless, the order of magnitude of the critical AP onset rapidness lies in the physiologically realistic range of the AP onset rapidness of real cortical neurons. It is therefore an interesting question whether this large AP onset rapidness of cortical neurons might have evolved in order to tune cortical networks near the edge of chaos.

\section{Benefits and Drawbacks of a Chaotic Dynamics}

A chaotic state is characterized by a permanent flow of information from the microstate to the macrostate due to the sensitivity to initial conditions [70]. This information flow is here quantified by the dynamical entropy production. Whether this entropy production is advantageous or disadvantageous for the information representation and processing in neural networks depends on the context.

Information in neural networks is encoded in the specific states of the composing neurons. The amount of information in these networks depends on the precision with which the state of the neurons can be read out. The maximal information capacity is then related to the attractor dimension $D$ as discussed above. Whether the entropy production in these networks overwrites the actual information or yields additional information depends on the precision of the read out of the network states.

On the one hand, if the network's precision is not optimal to encode all important information about the input, additional information can be gained due to the sensitivity to initial conditions. This can be thought of as a learning process about the network state, with increasing precision over time. Due to the sensitivity to initial conditions, differences in the network's microstate smaller than the given network precision separate exponentially over time and can be distinguished 
with the given precision at a later time. This creates information about a past microstate that would otherwise be inaccessible. We have provided a measure of this information gain with the rate of entropy production $H$. In fact, this mechanism could be physiologically relevant for odor discrimination in the olfactory bulb. Originally observed in zebrafish [106], it was discovered that while chemically evoked different odors initially lead to similar firing activity in mitral cells, such activity becomes progressively more distinct and hence more informative about the different odors over time [107].

On the other hand, if the network's precision is more or less optimal for encoding the input information, additional trivial information about the network's microstate would overwrite the more important information over time. Due to the information flow from the microstate to the macrostate, information would then be gained about irrelevant aspects of past microstates, e.g., ion channel noise. This gain of irrelevant information is also captured by the rate of entropy production $H$. Because we observed extensive dynamics in which the entropy production increases linearly with the number of neurons, it is well justified to define the average entropy production rate per neuron.

The strikingly high entropy production in networks of neurons with low AP onset rapidness described here suggests a rapid loss of input information in such neural networks. The average rate of entropy production in these chaotic neural networks is about 1 bit per spike per neuron. Interestingly, sensory input information provided to cortical networks is estimated to be about 1 bit per spike per neuron as well [93,94]. Therefore, if we assume the precision of the networks to be optimal for their task, this input information is overwritten by the intrinsic entropy production as quickly as it is encoded. Consequently, it would be difficult to maintain this information in cortical spike patterns for longer than a few spikes per neuron after stimulus onset. This suggests that maintaining relevant information in detailed network microstates beyond the immediate stimulus response would be quite unlikely. Such a picture is in agreement with a study of spike timing in rat barrel cortex [93], in which it was found that $83 \%$ of the information about whisker deflection is encoded in the first spike after the stimulus, and this information is reduced by two thirds with each successive spike.

Here again, it is a matter of debate whether a similar rate of incoming information and dynamic entropy production is advantageous or disadvantageous for the information processing in neural networks. On the one hand, a fast decorrelation of the input information at a similar rate as it is provided to the network would be advantageous for fast real time information processing where shadows from information about the past are only hindering optimal performance. On the other hand, the rapid loss of information at a similar rate as the incoming information would be disadvantageous if the network's task is to integrate information contained in longer spike sequences.

Importantly, we have found that the entropy production in chaotic networks decreases with increasing AP onset rapidness of the single neurons. In the experimentally expected relevant range for cortical neurons with a very large AP onset rapidness, this suggests a strongly decreased loss of information in such chaotic networks. Thus, the high AP onset rapidness of cortical neurons surprisingly reduces the information loss in chaotic cortical networks, which might be a strong reason why cortical neurons have evolved such a sharp AP initiation.

\section{Stable chaos}

The result of an edge of chaos in rapid theta neuron networks implies that in the limit of instantaneous AP initiation $(r \rightarrow \infty)$ the network dynamics can be stable. This is similar to networks of 
leaky integrate and fire neurons that exhibit stable chaos [4-7]. Such a form of stable dynamics despite an irregular network activity was shown to be fragile in terms of temporal synaptic transmission and finite size perturbations [5]. Because cortical neurons are expected to exhibit a very large AP onset rapidness, the further understanding of this stable chaos is of strong interest for the understanding of the functioning of cortical networks.

We therefore extended the introduced approach to precisely characterize the dynamics of leaky integrate and fire networks with temporally extended synaptic transmission. Our analysis reveals that incorporating temporally extended synaptic transmission, namely exponentially decaying synaptic currents, leads to a transition to extensive deterministic chaos (Chapter 4). The strength of chaos in such networks, however, is much weaker compared to the chaotic dynamics due to the active AP generation of theta neurons. The entropy production rate and attractor dimension are orders of magnitude smaller. Even though the activation of excitatory-inhibitory feedback loops in the networks intensifies the chaos, this intensification is quite moderate.

Our findings of a chaotic dynamics in the balanced state agree with accumulating evidence suggesting a highly sensitive dynamics in cortical networks, revealed by several in vivo experiments [101--104]. They showed that inducing one extra spike in a cortical neuron triggered a cascade of additional spikes in the network. The extra spikes are assumed to also induce missed spikes such that the network settles back to the previous statistically steady state. Nevertheless, the theoretically predicted stimulus-independent variations in the membrane potential, due to the ongoing missed and extra spikes, are predicted to be large and can be viewed as pure noise. This questions the use of a precise spike-timing code in cortical networks and suggests the use of a rate code [101].

Although the experimentally observed sensitivity to an extra spike is in line with our findings of a strongly chaotic dynamics in cortical networks, care must be taken when comparing the two scenarios. We investigated the dynamics with respect to infinitesimal spike-order preserving perturbations. Therefore, our results are expected to be representative of the impact of small enough perturbations. It is not obvious per se that this is the case for the single spike perturbations applied in the experiments. Such perturbations could in principle lead to different behavior. On the other hand, it is also not self-evident that systems that are insensitive to infinitesimal perturbations do not show sensitivity to single spike perturbations. Networks of neurons with instantaneous AP initiation were shown to exhibit stable dynamics for small enough spike-order preserving perturbations. Adding or removing single spikes, however, breaks this assumption. We therefore studied the dynamics in this interesting case in more detail.

Our analysis of inhibitory leaky integrate and fire networks with $\delta$ pulse coupling revealed that the local stability to infinitesimal perturbations coexists with a high sensitivity to single spike perturbations (Chapter 5). We have confirmed that the complete Lyapunov spectrum of such networks is negative definite, indicating stable dynamics with respect to infinitesimal perturbations. Additionally, we have shown that the Lyapunov spectra are also invariant to the network size, indicating extensive dynamics, and that in the large network and connectivity limit perturbations decay as fast as in uncoupled neurons. Surprisingly, the application of single spike perturbations revealed a completely unexpected behavior. Despite the stable dynamics to infinitesimal perturbations and despite a very weak rate response after single spike failures, such single spike perturbations lead to exponential state separation, causing complete decoherence of the network's microstate within a few milliseconds. The rate of exponential state separation was quantified with the so-called pseudo Lyapunov exponent that scales proportional to $K \bar{v}$ and thus appears to grow to infinity in the large network and connectivity limit. How can such an extreme sensitivity to single spike perturbations 
coexist with the local stability indicated by the negative definite Lyapunov spectrum?

The extended analysis of the dynamics of inhibitory leaky integrate and fire networks with respect to finite size-perturbations suggests the partitioning of the network's phase space into a tangle of stable flux tubes. States within these flux tubes are attracted to a unique, dynamically stable trajectory. Different flux tubes, however, separate exponentially fast. Interestingly, the flux tube radius (as a measure of the basin boundary that separates stable from unstable dynamics) decreases with the network size. This indicates that an unstable dynamics dominates the thermodynamic limit. The resulting sensitivity to initial conditions is then described by the pseudo Lyapunov exponent that showed no sign of saturation in the large connectivity limit.

These findings clarify the origin of the previous contradictory findings of local stability with negative Lyapunov exponents on the one hand [4-7] and extremely unstable dynamics with an infinite Lyapunov exponent on the other hand [2,3]. They arise in the limit of an instantaneous AP generation in the single neuron dynamics and result from a different order in which the limit of large network size and the limit of weak perturbations are taken. Taking the weak perturbation limit first results in a decaying perturbation within the flux tube around the current trajectory, even when taking the large network limit afterwards. Thus the thermodynamic limit would be characterized by negative Lyapunov exponents. In contrast, taking the large network limit first implies that vanishingly thin flux tubes and even infinitesimal weak perturbations would lead to an exponential state separation quantified by the pseudo Lyapunov exponent that shows no sign of saturation. Thus the thermodynamic limit would be characterized by an infinite Lyapunov exponent.

The picture of flux tubes in the phase space of such stable networks provides a basis for insensitivity to small perturbation (e.g. for noise or small input variations) and strong sensitivity to larger perturbations. This is interesting in terms of an error correcting code in neural networks and in the context of reservoir computing. Reservoir computing is the unifying term for the bottom-up attempt to construct classification and discrimination systems on the basis of such neural networks. The founding models of reservoir computing are the liquid-state machine [108] and the echo-state machine [109]. In this context, the flux tube radius defines a border between the fading property (variations of smaller initial conditions die out exponentially) and the separation property (larger input variations cause exponentially separating trajectories). Since leaky integrate and fire neurons are widely used in reservoir computing, these applications may strongly benefit if the flux tube structure of the network's phase space is taken into account.

\section{Weak Pairwise Correlations}

Interestingly, each of the networks studied here were found to exhibit extensive dynamics in the balanced state. Extensive dynamics is classically expected in spatially extended systems that can be decomposed into weakly interacting subsystems whose number grows linearly with the system size [110]. The union of the Lyapunov spectra of the subsystems then yields the spectrum of the whole system and a well-defined spectrum per unit volume exists in the thermodynamic limit [110]. Our analysis shows that such a limit and an extensive nature of the dynamics exists in the balanced state. This extensivity in contrast to the classical case has no trivial explanation, as we have studied networks with random graph topology. Classically, it is observed in systems exhibiting spatio-temporal chaos [111-114] characterized by fast spatial and temporal decorrelation. In systems with random graph topology, a spatial structure is difficult to define since all neurons are potentially neighbors and the size of the system (the graph diameter) scales only logarithmically with the number of elements. A possible explanation of the extensivity in balanced networks is the 
lack of pairwise correlations between neurons. Weak pairwise correlations imply weak pairwise interactions, such that all neurons can be considered equal and the collective network dynamics becomes invariant with respect to the number of neurons.

These weak pairwise correlations, however, do not imply vanishing higher order correlations. We have given heuristical arguments that the attractor dimension derived from the Lyapunov spectrum provides an estimate of the order of nonvanishing higher order correlations between the neurons. The fat chaotic attractors in networks with low AP onset rapidness then imply that these neurons are largely uncorrelated. Stimulus-dependent correlated activity of small groups of neurons after stimulus onset would then be very informative. Within a short time, the strong chaos in these networks would quickly lead back to the decorrelated responsive state. For large AP onset rapidness, the attractor dimension is strongly reduced, implying higher order correlations between the neurons. With increasing AP onset rapidness towards the edge of chaos, the neurons states are then more and more entangled. In this entangled state, correlated activity of only a few neurons would not yield information about the stimulus unless a certain sequence of spikes encodes a certain stimulus. Encoding information in precise, long complex spike sequences is in principle possible in a stable dynamics. This ability should, however, be contrasted or complemented with the sensitivity to single spike perturbations that we observed in such formally stable dynamics. It seems more plausible that such neural networks use a rate code.

\section{Transition from Asynchrony to Synchrony}

Beyond characterizing the dynamics of the balanced state, we also observed two phase transitions from the asynchronous state to a synchronous state in networks of neurons with low AP onset rapidness. In inhibitory networks, synchronous oscillations emerge when increasing the number of recurrent connections $K$ beyond a network size-independent critical connectivity. In the synchronous state, single neurons fire irregularly such that the network-averaged coefficient of variation was unaffected by this transition. The dynamics in this synchronous irregular state also remain chaotic. The critical connectivity $K_{c} \approx 200$ at which this phase transition occurs is seemingly insensitive to the number of neurons in the networks but increases strongly with the AP onset rapidness $r$, such that it disappears for already moderately large $r$. Such a transition at a network size-independent critical connectivity was previously observed in inhibitory networks of hippocampal interneurons modeled by the conductance-based Wang-Buzsáki model [38] and further investigated in leaky integrate and fire neuron networks [92]. We found that this transition also occurs in excitatory-inhibitory networks but at an even higher critical connectivity.

Additionally, we observed a different phase transition to a synchronous regular state while increasing the strength of the excitatory-inhibitory feedback loops in networks of neurons with low AP onset rapidness. This transition is characterized by stable dynamics and resembles a transition previously studied in networks of quadratic integrate and fire neurons but with all-to-all coupling [115]. For the sparse networks studied here, the characteristics of the oscillatory synchronous state was also described in networks of leaky integrate and fire neurons, conductance based WangBuzsáki neurons and exponential integrate and fire neurons [116, 117]. For moderately large AP onset rapidness, this transition also disappears. 


\subsection{Outlook}

We have mentioned in the introduction that three main factors play a fundamental role in the dynamics of neural networks: (i) the individual neuron dynamics, (ii) the individual synapse dynamics and (iii) the network topology. In this thesis the influence of single neuron properties and synaptic transmission were addressed. All studied networks had a random graph topology.

\section{Network Topology}

The presented approach can be directly applied to arbitrary network topologies and heterogeneous synaptic strengths. It thus lays the foundation for an investigation of the collective network dynamics depending on different graph structures, e.g., small world graphs or scale free graphs, but also any other local graph structure, e.g., with realistic spatially-extended structures and different subgraphs. Interesting in such future studies will be not only the dependence of the dynamical nature and the rate of entropy production but also the structure of the covariant Lyapunov vectors that might yield insight into the information flow in such networks. The influence of the flux tube radii on the local network structure should also be considered in this context. An increased flux tube radius for some specific firing sequences would indicate favored spike patterns depending on the local graph structure and synaptic strengths.

Our approach could therefore yield potential insight in terms of signal propagation modulated by underlying feed-forward substructures in otherwise random networks. This can be realized in the topology [118] or synaptic strengths [119]. It has been shown that the resulting non-normal networks exhibit extended memory capacity compared to normal networks [120]. The quantification of the information loss with the current approach may provide additional insight about the lifetime of memory traces in such networks. Completely different topologies, such as layered network structures can, of course, also be studied. The hierarchical organization of feedforward networks, for instance, has recently been accounted for the possibility of combining both sequential and simultaneous compositions of primitives through synfire chains [121]. The dynamical nature of such networks in which information is apparently reliably encoded in precise spike times remains to be precisely determined.

\section{Synaptic Transmission}

To further increase the physiological relevance of the studied networks, the effect of synaptic delays should be addressed. Leaky integrate and fire networks with [4-7] and without delays (Chapter 5) both exhibit a seemingly similar form of stable chaos. This suggests that a synaptic delay in these random networks with asynchronous activity might play a less important role than in, for example, all-to-all coupled networks with synchronous activity. This should be analyzed in future research. Including synaptic delays in the proposed approach must be executed carefully, as the phase space is then of varying dimensionality [86]. It should also be noted that the finite time blowup in the theta neuron model can be considered as an effective delay because the neurons are basically insensitive during this state. This effective delay is in the order of $1 \mathrm{~ms}$.

We have already analyzed the effect of temporally extended synaptic currents in leaky integrate and fire (LIF) networks. Because $\delta$ pulse coupled LIF networks exhibit stable dynamics, the arising chaos in LIF networks with exponentially decaying synaptic currents can be attributed to the temporally-spread synaptic currents. It would be interesting to investigate whether the incorpora- 
tion of exponentially decaying synaptic currents in theta neuron networks leads to a comparable increase of the chaos which would then be negligible or leads to a different effect. Existing analytic solutions of the theta neuron model with exponentially decaying synaptic currents [122] provide a good starting point for such an extension of our approach.

\section{Single Neuron Models}

Another important topic for future studies is to continue the investigation of the effect of different single neuron models on the collective dynamics. To this end, our approach is especially simple to apply if the phase-response curves of the single neurons are known exactly, as the single spike Jacobians are obtained in terms of their derivatives. A detailed analysis of the information loss depending on the neurons' phase-response curves related to the calculated information rate in Ref. [123] would further quantify the advantages and disadvantages of different single neuron dynamics for information processing.

If the phase-response curves cannot be derived analytically, e.g., for higher dimensional conductance-based neuron models or the exponential integrate and fire model, our approach can still be used. The phase-response curves should then be computed numerically. Their derivatives, used in the single spike Jacobians, could be provided in the form of numerical fits or lookup tables. High precision network simulations should be used to return the spike times and phases of the neurons. The Lyapunov spectra could be approximately calculated with the numerically obtained phase-response curves in the general procedure presented here.

It is, however, not clear how precise the characterization of the network dynamics would be with this procedure, because using only the phase-response of the first spike after a perturbation might be a poor approximation. The incorporation of higher order phase response curves would be one possible and quite challenging future extension of our approach. Studying the network dynamics with the approximation of only considering the response to the first spike after the perturbation can be attacked right away. This subject, however, requires further critical examination before networks of multidimensional single neuron models can be characterized solely based on their phase-response curve.

\section{Other Applications, Extensions and Implementations}

The presented approach should certainly be applied to network models that are known to accurately describe aspects of real cortical networks. The comparison of the dynamical entropy production in such networks to the actual sensory information content would yield fundamental insight for the understanding of such networks. An example for such a network model is the ring model [124]. A model accurately describing the rat barrel cortex would also be highly interesting because of the parallel conclusion drawn here of a rapid information loss in theta neuron networks and that from experimentally measured information loss in the rat barrel cortex [93]. Finding an appropriate model of the rat barrel cortex, however, will be a challenging initial task. Once this model is established, the application of our approach can be applied to compare the dynamical entropy production rate to the real information content in such networks.

Further analytical steps towards a better understanding of the network dynamics should also be taken into account. This is quite a challenging task for random graphs with an asynchronous activity. Further analysis of, e.g., the stability of the asynchronous splay state in all-to-all coupled networks could potentially explain the qualitatively different dynamical regimes of neural networks 
with different AP onset rapidness (see Appendix D). In order to address the network dynamics of random networks in the asynchronous balanced state, we have taken a semianalytic approach. The single neuron model was solved analytically leading to the exact single spike Jacobian. Then, we numerically calculated the Lyapunov spectra of such networks. It was also possible to derive a random matrix approximation of the mean Lyapunov exponent analytically. A similar approach or a generalization of the dynamical mean-field theory studied in Ref. [46] might be used to derive an upper bound of the largest Lyapunov exponent. With such a result the dynamics in the thermodynamic limit could be rigorously analyzed, complementing our numerical findings. The understanding of the scaling of the largest Lyapunov exponent with the number of neurons in rapid theta networks or the numerically observed logarithmic increase in correlated leaky integrate and fire networks is not yet exhausted, and would strongly benefit from an analytic derivation.

Other important aspects in terms of nonlinear dynamics are a extension of the analysis of the covariant Lyapunov vectors. Here, we started to investigate the hyperbolicity of the studied systems and introduced the characteristics of temporal network chaos in random theta neuron networks. This should be further examined together with the temporal and spatial correlation of the covariant Lyapunov vectors and local Lyapunov exponents.

Small theta neuron networks also provide an interesting type of conservative chaos. For example three neuron motifs can exhibit stable, quasiperiodic or chaotic dynamics depending on the topology and coupling strength. This should be analyzed in more detail, numerically and analytically. The route to chaos in such three neuron motifs but also in random networks of rapid theta neuron at the edge of chaos (see Fig. 3.24) should be characterized as well.

A demonstration of how large neural networks with a chaotic dynamics would perform under real conditions is another fundamentally important aspect that should be addressed in future research. Other work has already made considerable progress in this respect, such as the FORCE algorithm [125] and the promising research of reservoir computing [108, 109]. Besides quantifying the dynamical entropy production and analyzing the information flow in such networks, taking into account the flux tube picture in networks of neurons with instantaneous AP initiation may strongly benefit these applications.

The edge of chaos is a recurrent theme in neural computation. A similarly famous aspect is chaos control. Whether realistic computations can be achieved on short time scales on existing stable modes in a stable or chaotic state, or unstable modes are dynamically stabilized in a chaotic state remains an open and fascinating field of research.

A final point is the computational neuroscience aspect. The calculation of the Lyapunov spectra requires formidable computational power because the computation time of the necessary matrix orthogonalizations scales proportional to the cube of the system size. We have developed a highly performant parallel algorithm that allows for the calculation of the Lyapunov spectra of moderately large networks. This algorithm appears to be an ideal candidate to be used on graphics processing units (GPU). Such an implementation would enable a large community of scientists to use the proposed approach to accurately characterize the network dynamics without the need of a highperformance cluster. 


\section{Appendix A}

\section{Integrate and Fire Neuron Models}

The membrane of a neuron can be thought of as capacitor with voltage-dependent membrane currents. The membrane potential, or voltage, is then described by the differential equation

$$
C \frac{\mathrm{d} V}{\mathrm{~d} t}=I_{m}+I_{s}
$$

with the membrane capacitance $C$, the voltage-dependent membrane current $I_{m}$ and the synaptic input current $I_{s}$. The family of integrate and fire neuron models differs from standard conductancebased neuron models, in that they do not describe the dynamics of different ion channels in detail but try to capture the essential dynamics of the membrane potential in a one-variable model.

There are four different integrate and fire models:the perfect integrator (PIF) where the membrane current is zero, the leaky (also linear) integrate and fire model (LIF) where only a leak current is considered, the quadratic integrate and fire model (QIF) where the membrane current is a quadratic function of the voltage, and the exponential integrate and fire model (EIF) where a leak term and an exponential function constitute the membrane current. The voltage-dependent membrane currents of the integrate and fire models are [88]

$$
\begin{aligned}
I_{m}^{\mathrm{PIF}}(V) & =0 \\
I_{m}^{\mathrm{LIF}}(V) & =-g_{L}\left(V-V_{L}\right) \\
I_{m}^{\mathrm{QIF}}(V) & =\frac{g_{L}}{2 \triangle_{T}}\left(V-V_{T}\right)^{2}-I_{T} \\
I_{m}^{\mathrm{EIF}}(V) & =-g_{L}\left(V-V_{L}\right)+g_{L} \triangle_{T} \exp \left(\frac{V-V_{T}}{\triangle_{T}}\right)
\end{aligned}
$$

where $g_{L}$ is the leak conductance, and $V_{L}$ the leak potential. The rheobase current, the minimal input current for the neuron start firing is denoted $I_{T}$, the voltage at the minimum of the membrane current is $V_{T}=V_{L}+\triangle_{T}$ with the spike slope factor $\triangle_{T}$. (Fig. A.1). The passive membrane time constant is $\tau_{\mathrm{m}}=C / g_{L}$. These models are complemented with reset and threshold conditions. At a certain threshold, the neuron is said to fire an action potential. Afterwards, the membrane potential is reset to $V_{R}$, possibly after a refractory period $\tau_{r}$. Setting the reset and threshold potential to $\pm \infty$ in the QIF model leads to the theta neuron. The above equations have physiologically correct dimensions. 
A

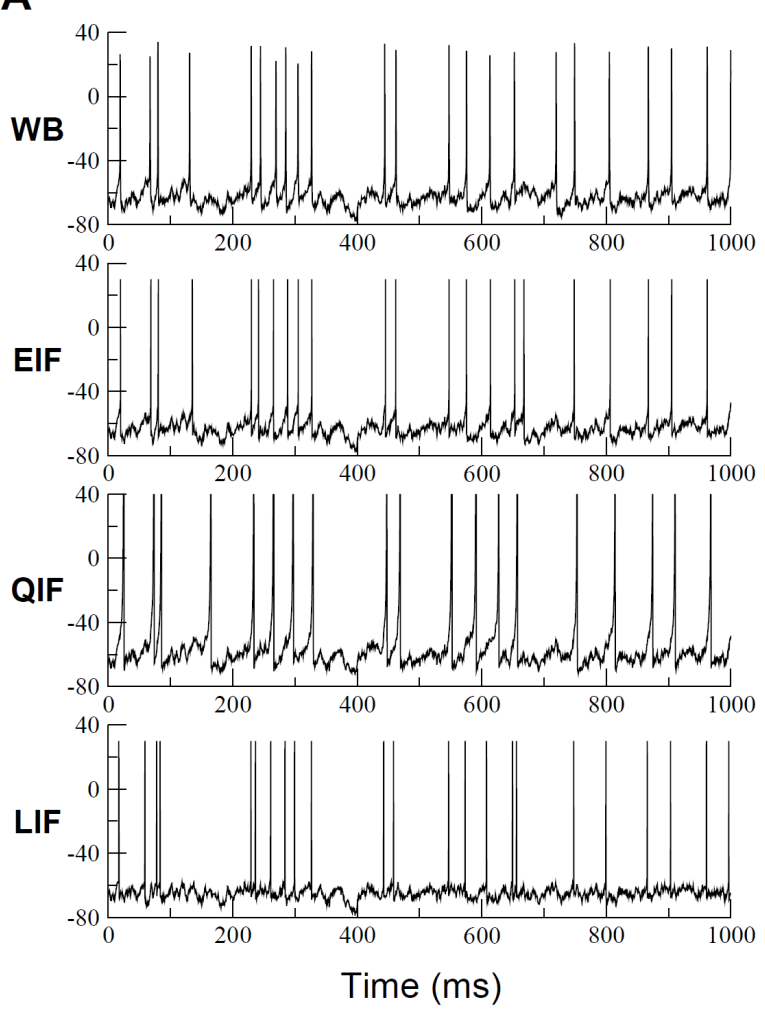

B

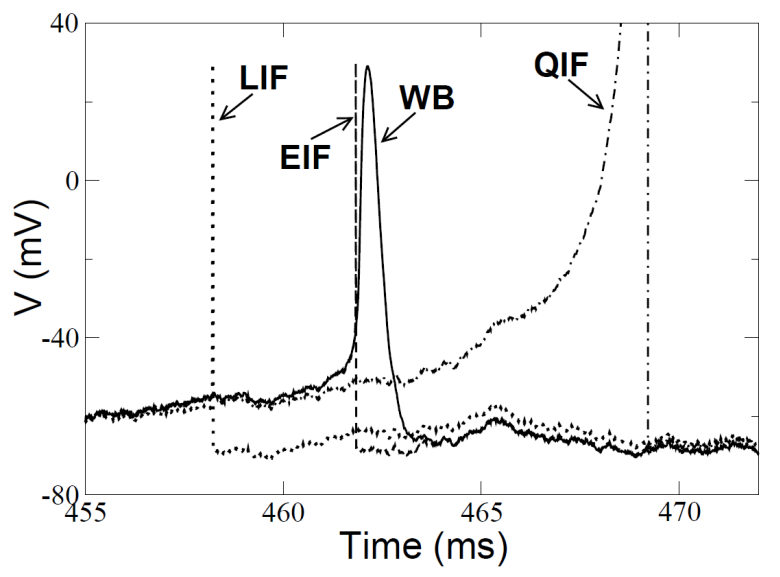

C

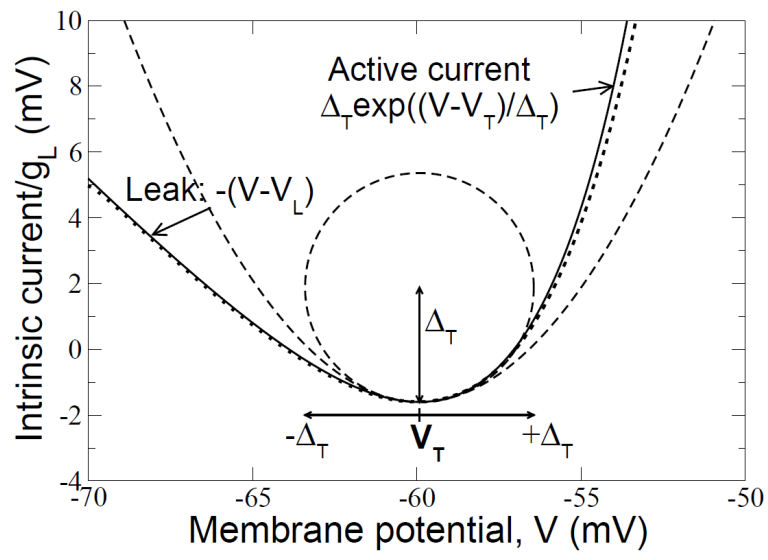

Figure A.1 - Integrate and fire neuron models. (Figures from Ref. [88]) A) Voltage traces for WB, EIF, QIF, and LIF neurons for the same realization of the noisy input current, B) shows a higher resolution for a short time interval in which a spike has been generated in all models. The subthreshold traces are similar for all models; however, the dynamics of the spike are different on a ms time scale. When the fluctuation leads to a spike in all models, the LIF neuron spikes first. The EIF neuron spikes almost exactly at the spike onset of the WB. The QIF neuron fires much later, C) $I V$ curve of the EIF (solid line) and WB (dotted line) neurons. The threshold $V_{T}$ is defined as the minimum of the curve. The spike slope factor $\triangle_{T}$ is proportional to the radius of the curvature of the $I V$ curve at its minimum. 
The stable fixed point of the LIF model is at $V_{S}=V_{L}$. It does not incorporate an action potential generating unstable fixed point. For the neuron to fire, the stable fixed point needs to be pushed by the rheobase current above the threshold.

The QIF model has two fixed points

$$
\begin{aligned}
\frac{\mathrm{d} V}{\mathrm{~d} t}=0 & =\frac{g_{\mathrm{L}}}{2 \triangle_{T}}\left(V^{*}-V_{T}\right)^{2}-I_{T} \\
V^{*} & =V_{T} \pm \sqrt{I_{T} \frac{2 \triangle_{T}}{g_{L}}} .
\end{aligned}
$$

The fixed point at $V_{\mathrm{S}}=V_{T}-\sqrt{I_{T} 2 \triangle_{T} / g_{L}}$ is stable and the one at $V_{\mathrm{U}}=V_{T}+\sqrt{I_{T} 2 \triangle_{T} / g_{L}}$ is unstable. The derivative of the voltage is set to $-1 / \tau_{\mathrm{m}}$ as in the LIF model, which sets the rheobase current $I_{T}$ in the QIF model:

$$
\begin{aligned}
\frac{\mathrm{d} J}{\mathrm{~d} V} \frac{\mathrm{d} V_{\mathrm{S}}}{\mathrm{d} t}=-\frac{1}{\tau_{\mathrm{m}}} & =\frac{g_{\mathrm{L}}}{C \triangle_{T}}\left(V_{S}-V_{T}\right) \\
& =-\frac{1}{\tau_{\mathrm{m}} \triangle_{T}} \sqrt{I_{T} \frac{2 \triangle_{T}}{g_{L}}} \\
I_{T} & =\frac{g_{L} \triangle_{T}}{2},
\end{aligned}
$$

and sets the stable and unstable fixed point $V_{\mathrm{S}, \mathrm{U}}=V_{T} \mp \triangle_{T}$.

The above equations are in physiologically correct dimensions. The transformation to a dimensionless voltage representation is obtained by

$$
\begin{aligned}
\bar{I} & =\frac{I}{2 \triangle_{T} g_{L}} \\
\bar{V} & =\frac{V-V_{T}}{2 \triangle_{T}}
\end{aligned}
$$

This transformation with $\tau_{\mathrm{m}}=C / g_{L}$ leads to

$$
\begin{aligned}
\tau_{\mathrm{m}} \frac{\mathrm{d} \bar{V}}{\mathrm{~d} t} & =\bar{I}_{m}+\bar{I}_{s} \\
\bar{I}_{m}^{\mathrm{LIF}} & =-\bar{V}-\frac{1}{2} \\
\bar{I}_{m}^{\mathrm{QIF}} & =\bar{V}^{2}-\bar{I}_{T} \quad \text { with } \bar{I}_{T}=\frac{1}{4} \\
\bar{I}_{m}^{\mathrm{EIF}} & =-\bar{V}-\frac{1}{2}+\frac{1}{2} \exp (2 \bar{V}) .
\end{aligned}
$$

This corresponds to the stable and unstable fixed point in the QIF model at $V_{S, U}=\mp 1 / 2$. In the LIF model, the stable fixed point is also $V_{S}=-1 / 2$ and the threshold would be set to $+1 / 2$. In the main part of the thesis, this was for simplicity shifted to 0 and 1 , respectively. 
Another commonly used representation is obtained by a transformation of the currents only:

$$
\tilde{I}=\frac{I}{C}=\frac{I}{\tau_{\mathrm{m}} g_{\mathrm{L}}} .
$$

This leads to a representation in which the voltage is still measured in $\mathrm{mV}$ but the currents are measured in $\mathrm{mV} / \mathrm{ms}$ :

$$
\begin{aligned}
\frac{\mathrm{d} V}{\mathrm{~d} t} & =\tilde{I}_{\mathrm{m}}(V)+\tilde{I} \\
\tilde{I}_{m}^{\mathrm{LIF}}(V) & =-\frac{1}{\tau_{\mathrm{m}}}\left(V-V_{L}\right) \\
\tilde{I}_{m}^{\mathrm{QIF}}(V) & =\frac{1}{2 \triangle_{T} \tau_{\mathrm{m}}}\left(V-V_{T}\right)^{2}-\tilde{I}_{T} \\
\tilde{I}_{m}^{\mathrm{EIF}}(V) & =-\frac{1}{\tau_{\mathrm{m}}}\left(V-V_{L}\right)+\frac{\triangle_{T}}{\tau_{\mathrm{m}}} \exp \left(\frac{V-V_{T}}{\triangle_{T}}\right) .
\end{aligned}
$$

\section{Fitting the Rapid Theta Neuron Model to the EIF}

In Chapter 3 the rapid theta neuron model with variable AP onset rapidness $r$ was introduced. It was built to yield the membrane time constant at the stable fixed point $V_{S}=-1 / 2$ and an $r$ times more rapid AP initiation at the unstable fixed point $V_{U}=1 / 2$. In the dimensionless voltage representation the rapid theta neuron model is defined as

$$
\tau_{\mathrm{m}} \frac{\mathrm{d} \bar{V}}{\mathrm{~d} t}= \begin{cases}a_{S}\left(\bar{V}-\bar{V}_{G}\right)^{2}-\bar{I}_{T}+\bar{I}(t) & V \leq V_{G} \\ a_{U}\left(\bar{V}-\bar{V}_{G}\right)^{2}-\bar{I}_{T}+\bar{I}(t) & V>V_{G}\end{cases}
$$

with

$$
\begin{aligned}
\bar{V}_{G} & =\frac{1}{2} \frac{r-1}{r+1} \\
\bar{I}_{T} & =\frac{1}{2} \frac{r}{r+1} \\
a_{S} & =\frac{r+1}{2 r} \\
a_{U} & =\frac{r(r+1)}{2}=r^{2} a_{S} .
\end{aligned}
$$

\section{FI-curves}

In this section, we investigate how the rapid theta neuron model can be fit to the exponential integrate and fire model (EIF). Originally, the EIF model was introduced by Fourcaud-Trocmé et al. [88] to fit the Wang-Buzsáki model of hippocampal interneurons [38]. It was, however, necessary to introduce a refractory period to fit the EIF to the Wang-Buzsáki model. They also fit the QIF model ( $r=1)$ with almost identical parameters but without a refrectory period to the Wang-Buzsáki model. The parameters obtained by Fourcaud-Trocmé et al. are summarized in 


\begin{tabular}{|c|c|c|c|}
\hline & EIF (Fourcaud-Trocmé [88]) & QIF (Fourcaud-Trocmé [88]) & EIF (Badel [126]) \\
\hline \hline$\tau_{\mathrm{m}}$ & $10 \mathrm{~ms}$ & $10 \mathrm{~ms}$ & $17.2 \mathrm{~ms}$ \\
\hline$g_{L}$ & $0.1 \mathrm{mS} / \mathrm{cm}^{2}$ & $0.1 \mathrm{mS} / \mathrm{cm}^{2}$ & - \\
\hline$V_{L}$ & $-65 \mathrm{mV}$ & - & $-57.0 \mathrm{mV}$ \\
\hline$V_{T}$ & $-59.9 \mathrm{mV}$ & $-59.9 \mathrm{mV}$ & $-42.0 \mathrm{mV}$ \\
\hline$\triangle_{T}$ & $3.48 \mathrm{mV}$ & $3.48 \mathrm{mV}$ & $1.51 \mathrm{mV}$ \\
\hline$\tau_{r}$ & $1.7 \mathrm{~ms}$ & - & $10 \mathrm{~ms}$ \\
\hline$V_{R}$ & $-68 \mathrm{mV}$ & $-63.8 \mathrm{mV}$ & $-55 \mathrm{mV}$ \\
\hline
\end{tabular}

Table A.1 - Model parameters of the exponential integrate and fire (EIF) model and the quadratic integrate and fire (QIF) model given in Ref. [88, 126]. The EIF and QIF models in the second and third column are fit to the Wang-Buzsáki model. The EIF model in the fourth column is fit to a measure $I V$-curve of a cortical neuron (see Fig. A.3).
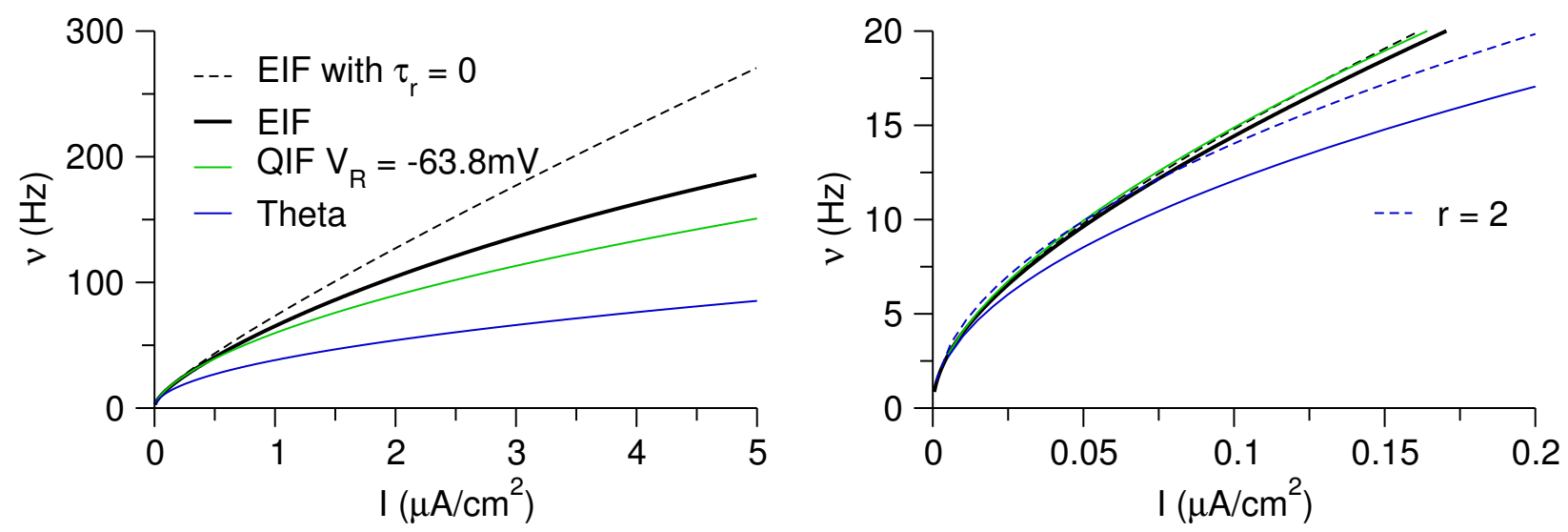

Figure A.2 - Tonic firing rates versus suprathreshold input current ( $F I$-curves) of different integrate and fire models. The EIF model with refractory period and the QIF model with finite reset are fit to the Wang-Buzsáki model in Ref. [88] (parameters in Table A.1). Additionally is shown the EIF model without refractory period and the theta neuron model and rapid theta neuron model with AP onset rapidness $r=2$.

Table A.1. Because the QIF model had a finite reset, it does not perfectly correspond to the theta neuron model used in Chapter 2. In fact, these subthreshold properties, such as refractory period and reset voltage strongly influence the $F I$-curve, to which the models were fit. In Figure A.2 are compared the models from Ref. [88] with two additional FI-curves of the EIF model without the refractory period and the QIF model with reset to $-\infty$ equivalent to the theta neuron model. We have also displayed the $F I$-curve of a rapid theta neuron with AP onset rapidness $r=2$. It can be seen, that the $F I$-curves of the different models and with different parameters differ considerably and it is questionable to use the FI-curve to obtain a good fit. The authors in Ref. [88] have used other measures for a comparison of the ability of a neuron to track fast changes in the input and came to the conclusion that the speed with which neurons can track changes in inputs is related to the spike slope factor determining the AP onset rapidness. These methods are, however, beyond the scope of this thesis but were applied to a similar neuron model in Ref. [127].

Another possibility for a fit is to look at the onset of the FI-curve. This is characteristic of the bifurcation that the neuron undergoes at the threshold to tonic firing. For type I excitable neurons this onset is $v \approx \beta \sqrt{I-I_{T}}$. The parameter $\beta$ for the EIF model is given in Ref. [88] and confirmed by the data in Fig. A.2 to $\beta=0.038 \mathrm{~ms}^{-1} \mu \mathrm{A}^{-1 / 2} \mathrm{~cm}$. For the rapid theta neuron model, 


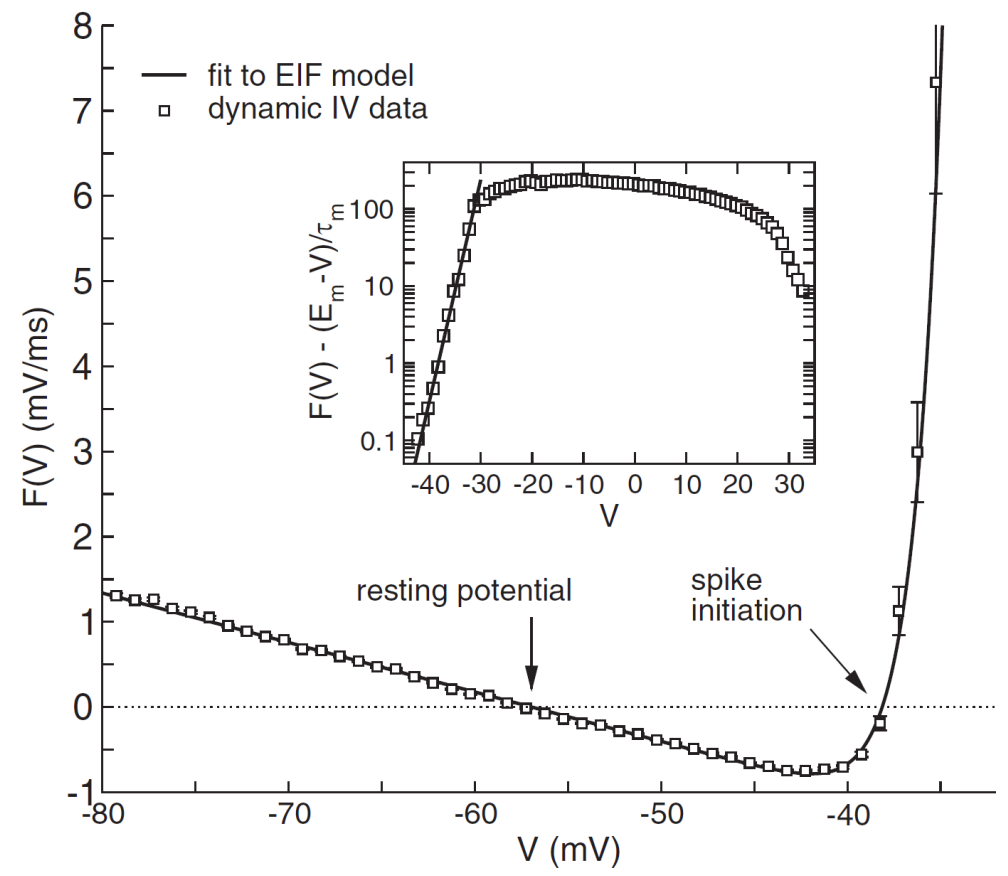

Figure A.3 - Experimentally obtained membrane current versus membrane potential ( $I V$-curve) of a cortical neuron and the fit to the EIF model from Ref. [126]. The parameters of the exponential integrate and fire (EIF) model are given in Table A.1.

the interspike interval, Eq. (3.11), and the conversion to the voltage representation, Eq. (A.2), yield

$$
\beta=\frac{1}{\pi \tau_{\mathrm{m}} \sqrt{\triangle_{T} g_{L}}} \sqrt{\frac{r}{r+1}}
$$

This solved for the AP onset rapidness $r$ yields

$$
\begin{aligned}
\frac{r}{r+1} & =\triangle_{T} g_{L} \pi^{2} \tau_{\mathrm{m}}^{2} \beta^{2} \\
r & =\frac{\triangle_{T} g_{L} \pi^{2} \tau_{\mathrm{m}}^{2} \beta^{2}}{1-\triangle_{T} g_{L} \pi^{2} \tau_{\mathrm{m}}^{2} \beta^{2}} .
\end{aligned}
$$

The parameters yield $r=1$, not too surprisingly because the EIF model and the QIF model share the same parameters in Ref. [88]. But we can check this with another EIF model that was fit to the experimentally obtained $I V$-curve of a cortical neuron.

Badel et al. measure the membrane current of a real cortical neuron and fit the parameters of the EIF model to match the data [126]. Their parameters are given in Table A.1. Because they use the third representation obtained by (A.5), we get

$$
\beta=\frac{1}{\pi \sqrt{2 \triangle_{T} \tau_{\mathrm{m}}}} \sqrt{\frac{r}{r+1}} .
$$

This solved for the AP onset rapidness $r$ yields

$$
r=\frac{\triangle_{T} \tau_{\mathrm{m}} \pi^{2} \beta^{2}}{1-\triangle_{T} \tau_{\mathrm{m}} \pi^{2} \beta^{2}}
$$



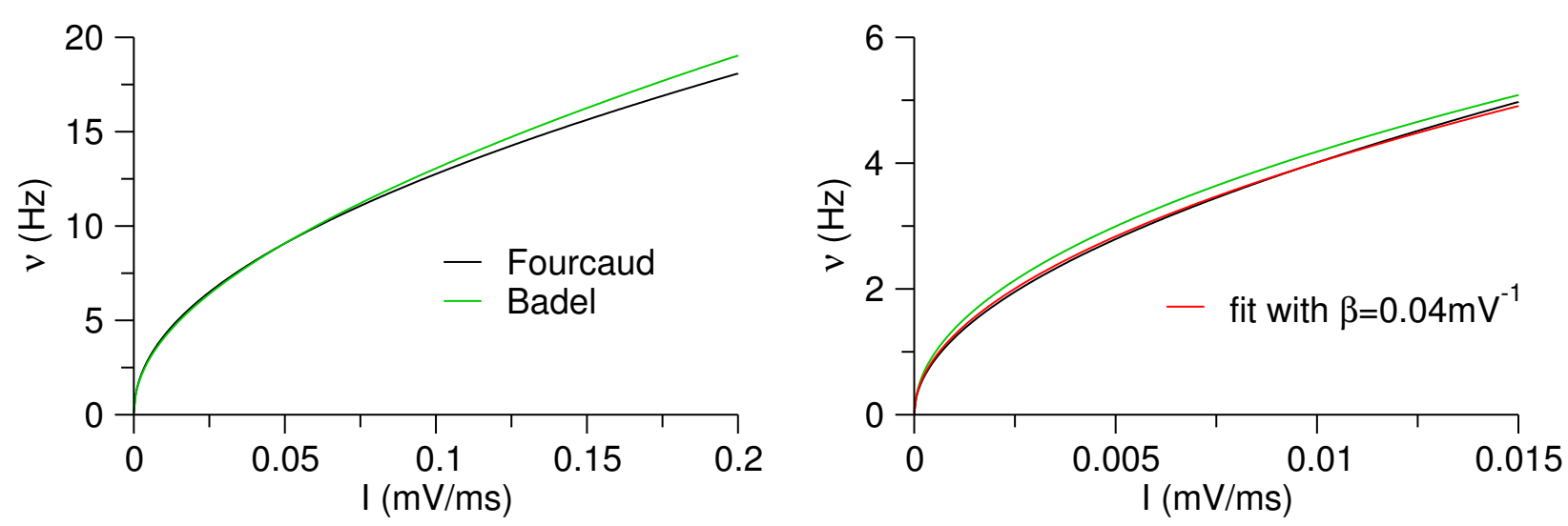

Figure A.4 - Tonic firing rates versus suprathreshold input current ( $F I$-curves) of the EIF model fit to the Wang-Buzsáki model $[\mathbf{8 8}]$ and to a cortical neuron [126]. The parameters of the exponential integrate and fire (EIF) model are given in Table A.1.

A numerical fit to the $F I$ curve of the EIF model with the parameters from Ref. [126] yields $\beta=0.040 \mathrm{mV}^{-1 / 2} \mathrm{~ms}^{-1 / 2}$, comparable to an AP onset rapidness $r=0.7$ (Fig. (A.4)).

\section{IV-curves}

Another possibility to fit the rapid theta neuron model to the EIF models from Ref. [88] and [126] is to use the membrane current directly. Because in the EIF model the membrane current $I_{m}$ grows exponentially with the voltage $V$, and in the rapid theta neuron model it grows quadratic, this is only representative at one specific voltage. We therefore measured the slope of the $I V$-curve at the stable and unstable fixed point. With the parameters from Fourcaud-Trocmé et al. [88], we get a ratio of $r=1.9$, whereas with the parameters from Badel et al. [126], we get $r=12$.

\section{Blowup time}

A third possibility to compare the different models is the finite time blowup from the unstable fixed point to threshold. This was measured with the neuron receiving the rheobase current, thus being just at the threshold to excitability. The blowup time of the rapid theta neuron model can be derived from Eq. (3.8) $T_{b}=2 \tau_{m} / r$. Comparing this with the numerically obtained blowup time of the two EIF models yields a corresponding AP onset rapidness of $r=2.7$ for the parameters from Fourcaud-Trocmé et al. [88] and $r=21$ for the parameters from Badel et al. [126]. 
A. Chapter Integrate and Fire Neuron Models 


\section{Appendix B}

\section{Minimal example for MATLAB}

The following code for MATLAB ${ }^{\circledR}$ demonstrates the event-based simulations and the calculation of the Lyapunov spectrum from the single spike Jacobians for random inhibitory theta neuron networks. Without including warmups, convergence etc., it is intended to illustrate the principle idea.

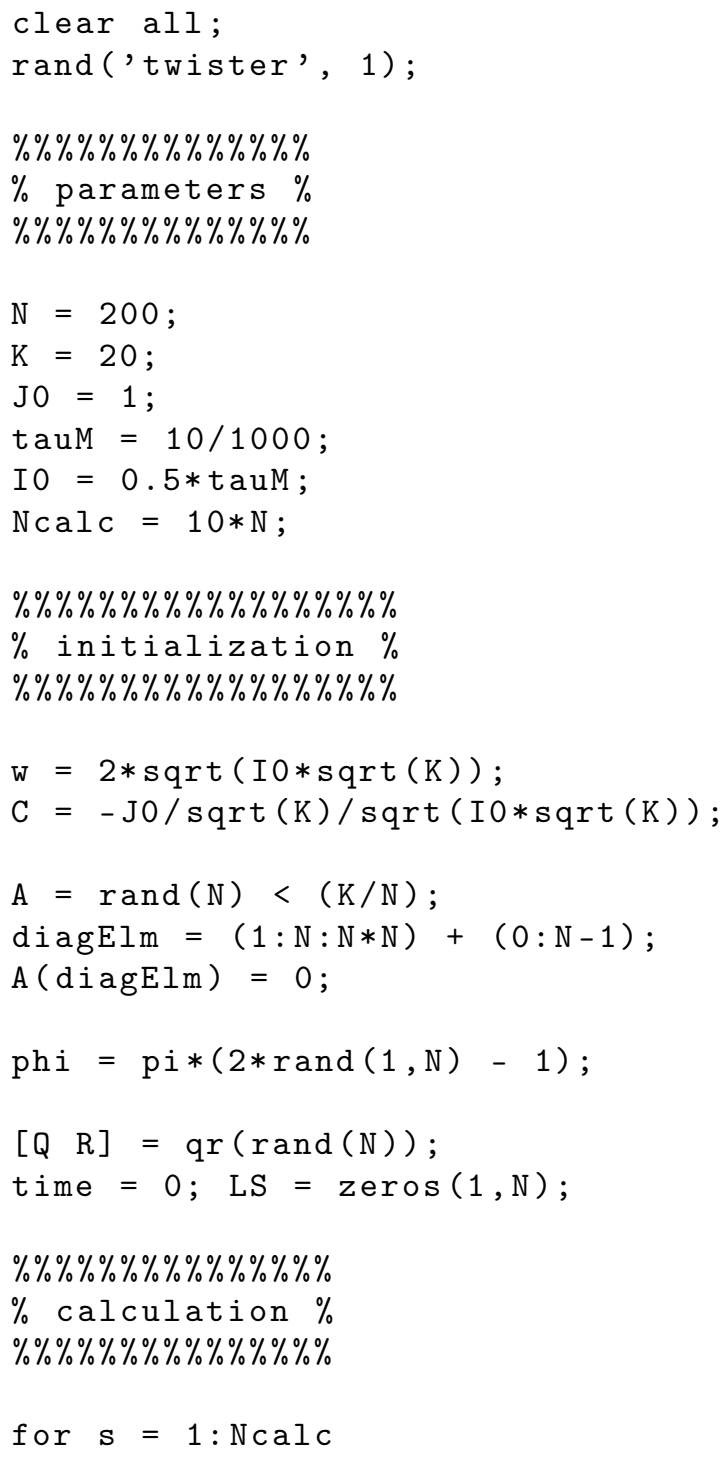

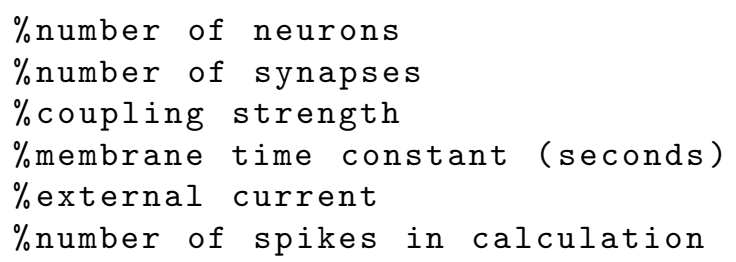




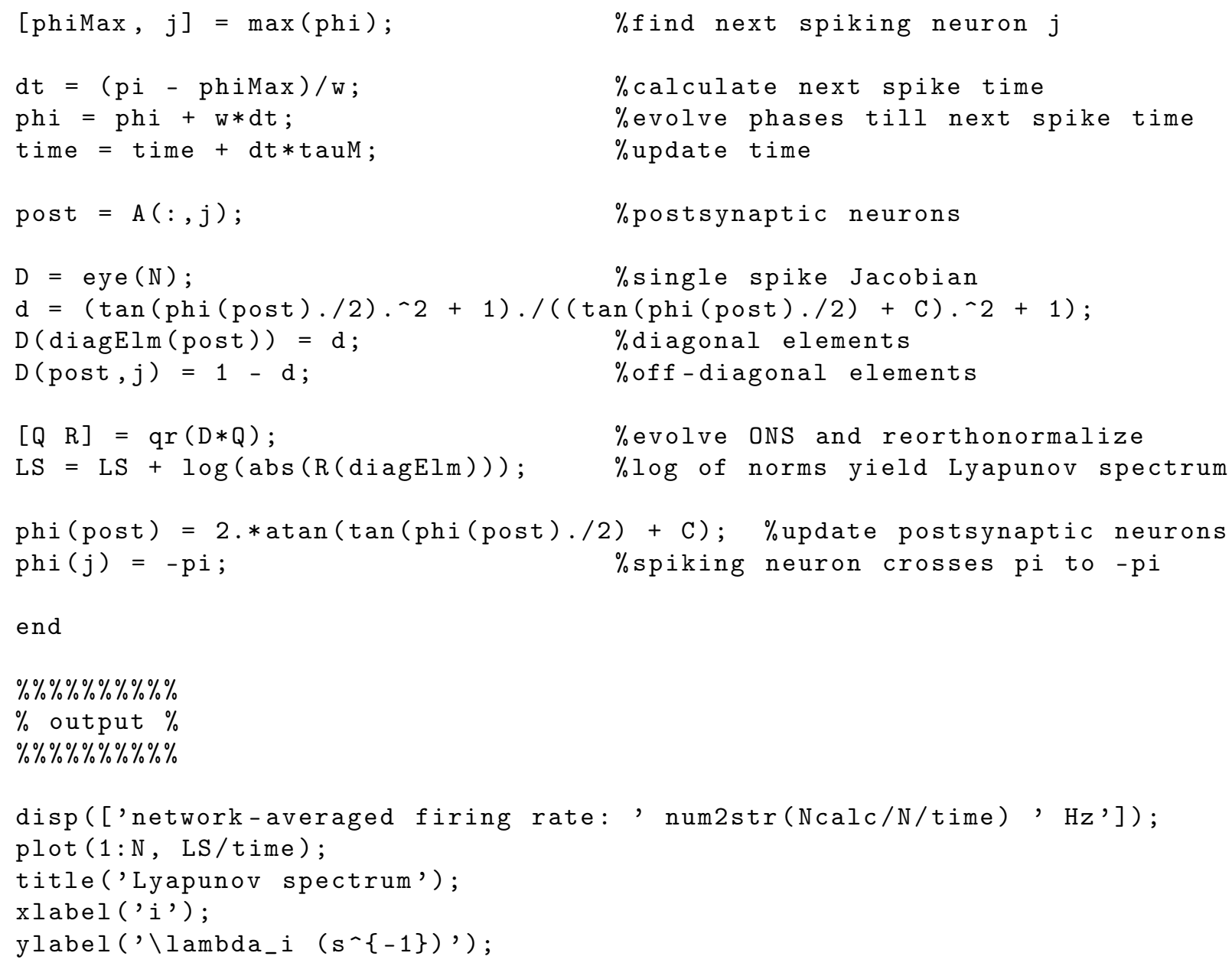




\section{Appendix C}

\section{Pairwise Spike Correlations}

Mathematically, the cross-correlation of two real functions $f$ and $g$ is defined as

$$
(f(t) \star g(t))(\tau)=\int f(t) g(t+\tau) \mathrm{d} t .
$$

It is closely related to the convolution of two functions:

$$
(f(t) * g(t))(\tau)=\int f(t) g(-t+\tau) \mathrm{d} t=\int f(-t+\tau) g(t) \mathrm{d} t
$$

While the cross-correlation (C.1) involves a shift and a multiplication, the convolution (C.2) involves reversing, then shifting and multiplying. If $X$ and $Y$ are two independent random variables with probability density functions $f$ and $g$ then the probability density of the difference $Y-X$ is given by the cross-correlation (C.1). In contrast, the convolution (C.2) yields the probability density function of the $\operatorname{sum} X+Y$.

The pairwise spike correlation of two neurons $i$ and $j$ with the spike trains $\left\{t_{i}\right\}$ and $\left\{t_{j}\right\}$ and the probability density functions $s_{i}(t)=\sum_{i} \delta\left(t-t_{i}\right)$ and $s_{j}(t)=\sum_{j} \delta\left(t-t_{j}\right)$ is

$$
\begin{aligned}
C(\tau) & =\left(s_{i}(t) \star s_{j}(t)\right)(\tau) \\
& =\left\langle s_{i}(t) s_{j}(t+\tau)\right\rangle_{t} \\
& =\int \delta\left(t-t_{i}\right) \delta\left(t-t_{j}+\tau\right) \mathrm{d} t \\
& =\sum_{i, j} \delta\left(t_{i}-t_{j}+\tau\right) .
\end{aligned}
$$

\section{Influence of Different Kernels}

Usually the calculation of pairwise spike correlations involves some kernel $K_{T}$ with a characteristic time scale $T$, that renders the probability density functions measurable. This kernel is convolved with the probability density functions leading to $s(t) \rightarrow K_{T}(t) * s(t)$. Typically used kernels are the box-function and the Gauss-function. The convolution with the Kernel around the spike times 
leads to

$$
\begin{aligned}
C_{T}(\tau) & =\left(\left(K_{T}(t) * s_{i}(t)\right) \star\left(K_{T}(t) * s_{j}(t)\right)\right)(\tau) \\
& =\sum_{i j}\left(K_{T}\left(t-t_{i}\right) \star K_{T}\left(t-t_{j}\right)\right)(\tau),
\end{aligned}
$$

where we evaluated the convolution of the kernels and the $\delta$ functions from the probability density function of the spike trains $i$ and $j$.

More generally the relationship between the $\delta$ correlation function $C(\tau)$ and the one obtained with finite kernels $C_{T}(\tau)$ will be derived using the following identities. The relation between correlation and convolution of two real functions $f, g$ is

$$
\begin{aligned}
(f(t) \star g(t))(\tau) & =\int f(t) g(t+\tau) \mathrm{d} t \stackrel{t \rightarrow-t}{=} \int f(-t) g(-t+\tau) \mathrm{d} t \\
& =(f(-t) * g(t))(\tau) .
\end{aligned}
$$

From Eq. (C.4) follows that for an even function $h$ with $h(-t)=h(t)$, the convolution and correlation are identical:

$$
(h(t) \star g(t))(\tau)=(h(t) * g(t))(\tau) .
$$

and

$$
\begin{aligned}
(h(t) * g(t))(-\tau) & =\int h(t) g(-t-\tau) \mathrm{d} t \stackrel{t \rightarrow t-\tau}{=} \int h(t-\tau) g(-t) \mathrm{d} t=\int h(-t+\tau) g(-t) \mathrm{d} t \\
& =(h(t) * g(-t))(\tau)
\end{aligned}
$$

Using these identities and associativity of the convolution $A *(B * C)=(A * B) * C$ yields:

$$
\begin{aligned}
C_{T}(\tau) & =\left(\left(K_{T} * s_{i}\right) \star\left(K_{T} * s_{j}\right)\right)(\tau) \\
& \stackrel{\text { C. } 4]}{=}\left(\left(K_{T} * s_{i}\right)(-t) *\left(K_{T} * s_{j}\right)(t)\right)(\tau) \\
& \stackrel{\text { C.6 }}{=}\left(\left(K_{T}(t) * s_{i}(-t)\right) *\left(K_{T}(t) * s_{j}(t)\right)\right)(\tau) \\
= & \left.\left(K_{T}(t) *\left(K_{T}(t) * s_{i}(-t)\right) * s_{j}(t)\right)\right)(\tau) \\
= & \left(\left(K_{T}(t) * K_{T}(t)\right) *\left(s_{i}(-t) * s_{j}(t)\right)\right)(\tau) \\
= & \left(\left(K_{T} * K_{T}\right) * C\right)(\tau)
\end{aligned}
$$

where the time-dependence was dropped for simplicity when identical for all functions. One can see from Eq. C.7 that the correlation of the kernels used in the spike trains influence the crosscorrelation.

The box-kernel is composed of two Heaviside step functions $\Theta$ centered at zero with width T:

$$
K_{T}^{\text {box }}(t)=\operatorname{Rect}(T)= \begin{cases}\frac{1}{T} & \text { for }-\frac{T}{2}<t<\frac{T}{2} \\ 0 & \text { otherwise }\end{cases}
$$


The Gauss-kernel is a Gaussian centered at zero with variance $\sigma^{2}=T$.

$$
K_{T}^{\text {Gauss }}(t)=\mathscr{N}(0, T)=\frac{1}{\sqrt{2 \pi T}} \exp \left(-\frac{t^{2}}{2 T}\right) .
$$

The corresponding convolutions (and cross correlations because they are even functions) are the triangle function and a Gaussian with twice the width:

$$
\begin{aligned}
\left(K_{T}^{\mathrm{box}} * K_{T}^{\mathrm{box}}\right)(t) & =\operatorname{Tri}(2 T)= \begin{cases}\frac{1-|t|}{T} & \text { for }-T<t<T \\
0 & \text { otherwise }\end{cases} \\
\left(K_{T}^{\text {Gauss }} * K_{T}^{\text {Gauss }}\right)(t) & =\mathscr{N}(0,2 T)=\frac{1}{\sqrt{4 \pi T}} \exp \left(-\frac{t^{2}}{4 T}\right) .
\end{aligned}
$$

The correlation functions with finite time bins thus relate to the $\delta$ correlation functions $C(\tau)$ as

$$
\begin{aligned}
C_{T}^{\text {box }}(\tau) & =[\operatorname{Tri}(2 T) * C](\tau) \\
& =\frac{1}{T} \int_{-T}^{T}(1-|t|) C(-t+\tau) \mathrm{d} t \\
C_{T}^{\text {Gauss }}(\tau) & =[\mathscr{N}(0,2 T) * C](\tau) \\
& =\frac{1}{\sqrt{4 \pi T}} \int_{-\infty}^{\infty} \exp \left(-\frac{t^{2}}{4 T}\right) C(-t+\tau) \mathrm{d} t .
\end{aligned}
$$

The minus sign in $C(-t+\tau)$ could be dropped since the convolved kernels are even functions.

Summarizing, the finite time kernels thus lead to an integration of the $\delta$ correlation functions around $\tau$ which is an effective smoothing of the functions. One should choose the width of the kernels smaller than the correlation time to avoid an over-averaging.

\section{Different Coefficients}

The most common correlation coefficient is the Pearson correlation coefficient $\rho$, which measures the linear relationship between two variables $X$ and $Y$. It is defined as

$$
\rho=\frac{\operatorname{COV}(X, Y)}{\sqrt{\sigma_{X}^{2} \sigma_{Y}^{2}}}
$$

where $\operatorname{COV}(X, Y)=\langle(X-\bar{X})(Y-\bar{Y})\rangle$ denotes the covariance and $\sigma_{X}^{2}=\left\langle(X-\bar{X})^{2}\right\rangle$ the variance. The angular brackets $\langle\ldots\rangle=\int \ldots \mathrm{d} t$ denote time average. The Pearson correlation coefficient is bound between \pm 1 ( +1 for perfect linearly increasing relation, -1 for a perfect linearly decreasing relation).

Also often used in neuroscience is a variant of the Pearson coefficient:

$$
c=\frac{\operatorname{COV}(X, Y)}{\bar{X} \bar{Y}}
$$

This correlation coefficient has a lower bound at 0 but no upper bound due to the different normalization compared to Pearson coefficient by the means $\bar{X}, \bar{Y}$. 
The relationships between these two correlation measures $(\overline{C .10})$ and $(\overline{C .11})$ and the aforementioned correlation $C=X \star Y$ are:

$$
\begin{aligned}
\rho & =\frac{C-\bar{X} \bar{Y}}{\sigma_{X} \sigma_{Y}} \\
c & =\frac{C-\bar{X} \bar{Y}}{\bar{X} \bar{Y}} \\
\rho & =c \frac{\bar{X} \bar{Y}}{\sigma_{X} \sigma_{Y}} .
\end{aligned}
$$

where we used the expression of the covariance $\operatorname{COV}(X, Y)=\langle(X-\bar{X})(Y-\bar{Y})\rangle=X \star Y-\bar{X} \bar{Y}$. Because the variances are the autocorrelations at zero $\left(\sigma_{X}^{2}=\left\langle(X-\bar{X})^{2}\right\rangle=X \star X-\bar{X}^{2}\right)$, the two correlation measures (C.10) and (C.11) can both be evaluated from the correlation $C$.

Evaluated with a box-kernel they are usually referred to as count-correlations. The counts of the spikes in a time bin of size $T$ are

$$
n_{i}=K_{T}^{\mathrm{box}} * s_{i} .
$$

Since the box-kernel is normalized here, the mean spike count is identical to the average firing rate $\bar{n}_{i}=\bar{v}_{i}$. Hence, the count correlation coefficient (C.11) becomes

$$
\begin{aligned}
c(\tau) & =\frac{\left\langle\left(n_{i}(t)-\bar{v}_{i}\right)\left(n_{j}(t+\tau)-\bar{v}_{j}\right)\right\rangle}{\bar{v}_{i} \bar{v}_{j}} \\
& =\frac{\left\langle n_{i}(t) n_{j}(t+\tau)\right\rangle}{\bar{v}_{i} \bar{v}_{j}}-1 \\
& =\frac{C_{T}^{\text {box }}(\tau)}{\bar{v}_{i} \bar{v}_{j}}-1 .
\end{aligned}
$$

The count Pearson coefficient (C.10) with Eq. (C.12) becomes

$$
\rho(\tau)=\frac{C_{T}^{\mathrm{box}}(\tau)}{\sigma_{i} \sigma_{j}}-\frac{\bar{v}_{i} \bar{v}_{j}}{\sigma_{i} \sigma_{j}}
$$




\section{Appendix D}

\section{Analytic Stability Analysis}

In this section we will examine the stability of some special firing patterns in all-to-all coupled networks based on the phase response curve of the single neurons (see, e.g., [87] for a review). For simplicity, all neurons are identical and couplings between neurons are homogeneous and inhibitory. A single neuron is purely deterministic and described by a phase defined on the unit circle $\phi \in[-\pi, \pi]$. When the neuron crosses $\pi \rightarrow-\pi$, the neuron is said to fire and emits a spike. The phase of the neuron describes its relative state compared to the unperturbed free-running neuron. Let us denote the interspike interval of the free-running neuron $T^{\text {free }}$. Then, the phase of the neuron is $\phi=\frac{T^{\text {free }}-T}{T_{\text {free }}}$ where $T$ denotes the potential time to its next spike based on the current state. If a neuron receives an input, the next spike time will be delayed or advanced 1 . This change, corresponding to a phase-change of the neuron, is captured by the phase-response curve $Z(\phi)=\frac{T-T^{\prime}}{T_{\text {free }}}$, where $T^{\prime}$ denotes the shifted next spike time of the neuron due to the received input. The phase-response curve is positive if the next spike time is advanced and negative if the next spike time is delayed. The phase-transition curve $g(\phi)=\phi+Z(\phi)$ describes the updated phase of the neuron after receiving an input. Figure D.1 shows an example of the phase-response and phase-transition curves of theta neurons, respectively.

\footnotetext{
${ }^{1}$ If the input has a temporal form, it might also influence successive spike times. This defines higher phase-response curves, but we will not be considering this case here. For $\delta$ pulse inputs the higher phase-response curves would vanish, for other temporal transmission functions their contribution can generally be assumed to be small.
}
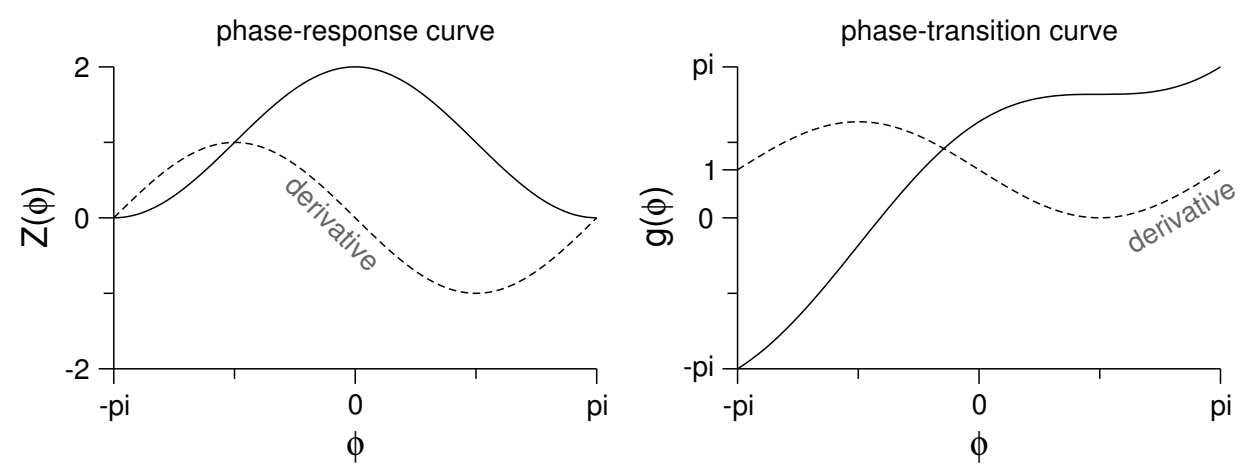

Figure D.1 - Example phase-response curve $Z(\phi)=1+\cos \phi$ and phase-transition curve $g(\phi)=\phi+$ $Z(\phi)$ of type I excitable neurons. The dashed lines are their derivatives. 


\section{Stable Periodic Firing of Two Theta Neurons}

The periodic firing of two mutually coupled neurons is one of the exactly solvable special cases. The following derivation can be similarly found in Ref. [128]. The two neurons are described by

$$
\begin{aligned}
& \dot{\phi}_{1}=\omega+Z\left(\phi_{1}\right) \delta\left(\pi-\phi_{2}\right) \\
& \dot{\phi}_{2}=\omega+Z\left(\phi_{2}\right) \delta\left(\pi-\phi_{1}\right) .
\end{aligned}
$$

The neurons phases evolve with constant phase-velocity $\omega$ between spike-events. Thus their phases evolve according to $f\left(\phi_{i}, t\right)=\phi_{i}+\omega t$, which also defines the time to the next spike $\triangle t\left(\phi_{i}\right)=\frac{\pi-\phi_{i}}{\omega}$. When receiving a spike from the other neuron, the spike-receiving neuron's phase shifts by an amount given by the phase response-curve $Z(\phi)$, respectively the phase is updated with the phasetransition curve $g(\phi)$. The spike times of the neurons are denoted $\left\{t_{1}^{(s)}\right\}$ and $\left\{t_{2}^{(s)}\right\}$. The evolution of the neurons' phases is summarized in the following table (we start with neuron 2 having just emitted a spike and neuron 1 at an initial phase $\phi$ ):

\begin{tabular}{|c|c|c|}
\hline$t$ & $\phi_{1}(t)$ & $\phi_{2}(t)$ \\
\hline \hline$t_{2}^{(1)}=0$ & $\phi$ & $-\pi$ \\
\hline$t_{1}^{(1)}=t_{2}^{(1)}+\frac{\pi-\phi}{\omega}$ & $\pi \rightarrow-\pi$ & $-\phi \rightarrow g(-\phi)$ \\
\hline$t_{2}^{(2)}=t_{1}^{(1)}+\frac{\pi-g(-\phi)}{\omega}$ & $-g(-\phi) \rightarrow g(-g(-\phi))$ & $\pi \rightarrow-\pi$ \\
\hline
\end{tabular}

Assuming a periodic spike sequence of period 1 leads to the condition that $\phi=g(-g(-\phi))$. Higher order periods of order $n$ are also possible and would lead to the condition $-\phi=(-g)^{2 n}(\phi)$. The following analysis focuses on the period 1 case but the generalization to higher periods would be straightforward.

We define the function $G(\phi)=g(-g(-\phi))$. The fixed points of this function are possible solutions of a periodic spike sequence. The phase-transition curve is generally invertible (monotonically increasing $g^{\prime}(\phi)>0$ ). The periodic solutions can thus be obtained from

$$
\begin{aligned}
G\left(\phi^{*}\right) & =\phi^{*} \\
-g\left(-\phi^{*}\right) & =g^{-1}\left(\phi^{*}\right) .
\end{aligned}
$$

Further analysis needs an explicit phase-transition curve. For the theta neuron the phase-transition curve is

$$
\begin{aligned}
g(\phi) & =2 \arctan \left(\tan \frac{\phi}{2}+C\right) \\
g^{-1}(\phi) & =2 \arctan \left(\tan \frac{\phi}{2}-C\right) \\
& =-g(-\phi) .
\end{aligned}
$$

This means that for the theta neuron the condition for the existence of a periodic solution (D.1) is fulfilled for any initial phase $\phi$. It also means that any phase difference of the two neurons is a periodic solution, not only for example the synchronous case. Next, the stability of this solution will be analyzed. 
The fixed point $\phi^{*}$ of $G$ is linearly stable if the derivative of $G$ is smaller than one. Then a perturbation from $\phi^{*}$ will decay. The condition for stability is

$$
G^{\prime}\left(\phi^{*}\right)=g^{\prime}\left(-g\left(-\phi^{*}\right)\right) \cdot g^{\prime}\left(-\phi^{*}\right)<1 .
$$

It is the product of the phase-transition curve evaluated at the phases of the two neuron at the times when they receive the spike from the other neuron. For the theta neuron, the derivatives are

$$
\begin{aligned}
g^{\prime}(\phi) & =\frac{\left(\tan \frac{\phi}{2}\right)^{2}+1}{\left(\tan \frac{\phi}{2}+C\right)^{2}+1} \\
g^{\prime}(-g(\phi)) & =\frac{\left(-\tan \frac{\phi}{2}-C\right)^{2}+1}{\left(-\tan \frac{\phi}{2}\right)^{2}+1} \\
& =\frac{1}{g^{\prime}(\phi)} .
\end{aligned}
$$

Therefore, $G^{\prime}(\phi)=1$ for all phases. We conclude that all periodic solutions are neutrally stable in the case of two mutually coupled theta neurons.

\section{Synchronous State in All-To-All Coupled Networks}

Another exactly solvable case is the synchronous state in all-to-all coupled networks. The derivation is similar to the two neuron case of the last section and can also be found in Ref. [128]. We start with the assumption that $N-1$ neurons are already synchronized and look at the one neuron that is not part of the synchronized cluster. The following table summarizes the evolution of the phase of the one not-synchronized neuron $\phi_{1}$ and all other already synchronized neurons $\phi_{N-1}$ :

\begin{tabular}{|c|c|c|}
\hline$t$ & $\phi_{1}(t)$ & $\phi_{N-1}(t)$ \\
\hline \hline$t_{2}^{(1)}=0$ & $\phi$ & $-\pi$ \\
\hline$t_{1}^{(1)}=t_{2}^{(1)}+\frac{\pi-\phi}{\omega}$ & $\pi \rightarrow-\pi$ & $-\phi \rightarrow g(-\phi)$ \\
\hline$t_{2}^{(2)}=t_{1}^{(1)}+\frac{\pi-g(-\phi)}{\omega}$ & $-g(-\phi) \rightarrow g^{N-1}(-g(-\phi))$ & $\pi \rightarrow-\pi$ \\
\hline
\end{tabular}

We see, that the difference to the two-neuron case is the phase update of the not-synchronized neuron due to the $N-1$ synchronized inputs. Its phase update is the composition of $N-1$ successive updates, as we assume that the spikes are still received in a specific order $g^{N-1}=g \circ \ldots \circ g$. Another possibility would be that the received $N-1$ spikes at the same time result in a $N-1$ times higher coupling strength. For the theta neuron, these two interpretations are in fact identical 
$g^{N}=2 \arctan \left(\tan \frac{\phi}{2}+N C\right){ }^{2}$, A periodic solution exists if

$$
\begin{aligned}
G\left(\phi^{*}\right)=g^{N-1}\left(-g\left(-\phi^{*}\right)\right) & =\phi^{*} \\
-g\left(-\phi^{*}\right) & =\left(g^{N-1}\right)^{-1}\left(\phi^{*}\right) .
\end{aligned}
$$

For general phase-response curves, including theta neurons, this will be fulfilled for $\phi^{*}= \pm \pi$, where the phase-response curve should vanish. This corresponds to the fully synchronous state since the one not yet synchronous neuron will approach the synchronous group from either behind or ahead. The question remains, whether the synchronous state with $\phi^{*}= \pm \pi$ is stable.

If the derivative of $G$ is smaller than one, the synchronous state is stable:

$$
G^{\prime}\left(\phi^{*}\right)=\left(g^{N-1}\right)^{\prime}\left(-g\left(-\phi^{*}\right)\right) \cdot g^{\prime}\left(-\phi^{*}\right)<1 .
$$

This depends on the derivative of the phase transition curves. For the theta neuron model, one gets

$$
\begin{aligned}
\left.\left(g^{N-1}\right)^{\prime}(\phi)\right) & =\frac{\left(\tan \frac{\phi}{2}\right)^{2}+1}{\left(\tan \frac{\phi}{2}+(N-1) C\right)^{2}+1} \\
\left(g^{N-1}\right)^{\prime}(-g(-\phi)) & =\frac{\left(\tan \frac{\phi}{2}-C\right)^{2}+1}{\left(\tan \frac{\phi}{2}+(N-2) C\right)^{2}+1} \\
g^{\prime}\left(-\phi^{*}\right) & =\frac{\left(\tan \frac{\phi}{2}\right)^{2}+1}{\left(\tan \frac{\phi}{2}-C\right)^{2}+1} \\
G^{\prime}(\phi) & =\frac{\left(\tan \frac{\phi}{2}\right)^{2}+1}{\left(\tan \frac{\phi}{2}+(N-2) C\right)^{2}+1} .
\end{aligned}
$$

At the synchronous fixed point $\phi^{*}= \pm \pi$, we find $G^{\prime}\left(\phi^{*}\right)=1$. Thus, the synchronous state in all-to-all coupled theta neuron networks is neutrally stable. This result can be generalized if the synchronous input of several neurons merely leads to an increase of the coupling strength, and the slope of the phase-response curve at $\pm \pi$ vanishes. In these quite general cases, the synchronous state in all-to-all coupled networks is neutrally stable. For other cases, see Ref. [128]. To further understand whether the synchronous state appears when neutrally stable, one should study the stability of the asynchronous state. If the asynchronous state is stable, the network will not reach a synchronous state. The stability of the asynchronous state is, however, much more complicated as will become clear in the next section.

${ }^{2}$ which can be shown by complete induction:

$$
\begin{aligned}
g & =2 \arctan \left(\tan \frac{\phi}{2}+C\right)=g^{1} \\
g \circ g^{N-1} & =2 \arctan \left(\tan \frac{\phi}{2}+(N-1) C+C\right)=g^{N} .
\end{aligned}
$$




\section{Splay State in All-To-All Coupled Networks}

An asynchronous state in all-to-all coupled networks is the so-called splay state, characterized by identical time intervals between successive spikes in the networks. The existence and stability of the splay state was investigated in Ref. [129,130]. It was shown in Ref. [130] that the splay state is stable in networks of neurons with a phase-response curve of negative slope $Z^{\prime}(\phi)<0$ for all phases. Then perturbations around the splay state decay. If the phase-response curve has positive slope $Z^{\prime}(\phi)>0$ for all phases, then perturbations around the splay state would grow and the splay state would be unstable. If the phase response curve has both positive and negative slope, like for theta neurons, one might conclude that the sum of expanding and contracting perturbations of the splay state determines its stability [131]. We will explain this idea for all-to-all coupled theta neuron networks.

In the splay state, the time between successive spikes in the networks is $\triangle t=T / N$, where $N$ is the number of neurons and $T=\omega /(2 \pi)$ is the interspike interval of one neuron. If the neurons were indistinguishable, the network's state would be identical at all spike times. We can assume the neurons to be ordered in increasing phase such that just after spike time $t_{s}$ the phases are $\phi_{i}\left(t_{s}\right)<\phi_{i+1}\left(t_{s}\right)$ for $i=1 \ldots N-1$ and the $N$-th neuron has just spiked, thus it was reset to $\phi_{N}=-\pi$. After the next spike time $t_{s+1}$ the phases have evolved to $\phi_{i}\left(t_{s+1}\right)=\phi_{i+1}\left(t_{s}\right)$ for $i=1 \ldots N-1$ and $\phi_{N}\left(t_{s+1}\right)=\phi_{1}\left(t_{s+1}\right)$. For $N=3$ this is demonstrated in the follwing table:

\begin{tabular}{|c|c|c|c|}
\hline$t$ & $\phi_{1}(t)$ & $\phi_{2}(t)$ & $\phi_{3}(t)$ \\
\hline \hline 0 & $\phi_{1}$ & $\phi_{2}$ & $-\pi$ \\
\hline$\frac{T}{3}$ & $g\left(\phi_{1}+\frac{\omega T}{3}\right)=\phi_{2}$ & $-\pi$ & $g\left(-\pi+\frac{\omega T}{3}\right)=\phi_{1}$ \\
\hline$\frac{2 T}{3}$ & $-\pi$ & $g\left(-\pi+\frac{\omega T}{3}\right)=\phi_{1}$ & $g\left(\phi_{1}+\frac{\omega T}{3}\right)=\phi_{2}$ \\
\hline$T$ & $\phi_{1}$ & $\phi_{2}$ & $-\pi$ \\
\hline
\end{tabular}

It can be seen that this is a consistent solution. The splay state is characterized by the fixed point $\vec{\phi}^{*}=\left(\phi_{1}, \ldots,-\pi\right)$ and the stability of this fixed point is determined by the slopes of the phase-transition curve at $\vec{\phi}^{*}$. Expressing the phase-transition curve $g(\phi)$ in terms of the phaseresponse curve $Z(\phi)$ we get $g^{\prime}(\phi)=1+Z^{\prime}(\phi)$. If the slopes of the phase-response curve at $\vec{\phi}^{*}$ are all negative, the splay state would be stable and if they are all positive it would be unstable. In the generic case of positive and negative slopes, perturbations would grow and shrink along the splay cycle and the splay state would only be stable if the sum of the slopes was negative [131].

With the infinitesimal phase-response curve of the theta neuron model $Z(\phi)=C(1+\cos \phi)$, thus $g(\phi)=\phi+C(1+\cos \phi)$, we get the splay state in all-to-all coupled networks of three neurons:

$$
\begin{aligned}
\phi_{1} & =g\left(-\pi+\frac{\omega T}{3}\right) \\
& =-\pi+\frac{\omega T}{3}+C\left(1+\cos \left(-\pi+\frac{\omega T}{3}\right)\right) \\
& \stackrel{C}{\simeq}-\frac{\pi}{3}+\frac{3 C}{2}+\mathscr{O}\left(C^{2}\right)
\end{aligned}
$$




$$
\begin{aligned}
\phi_{2} & =g\left(\phi_{1}+\frac{\omega T}{3}\right) \\
& \approx g\left(\frac{\pi}{3}+\frac{3 C}{2}\right) \\
& =\frac{\pi}{3}+\frac{3 C}{2}+C\left(1+\cos \left(\frac{\pi}{3}+\frac{3 C}{2}\right)\right) \\
& \stackrel{\longrightarrow}{\simeq} \frac{\pi}{3}+3 C+\mathscr{O}\left(C^{2}\right) \\
\phi_{3} & =-\pi,
\end{aligned}
$$

where we assumed $\frac{\omega T}{3} \rightarrow \frac{2 \pi}{3}$ for $C \rightarrow 0$. The derivative of the phase-response curve is $Z^{\prime}(\phi)=$ $-C \sin \phi$, thus

$$
\begin{aligned}
Z^{\prime}\left(\phi_{1}\right) & =-C \sin \left(-\frac{\pi}{3}+\frac{3 C}{2}\right) \\
& \approx C\left(\frac{\sqrt{3}}{2}-\frac{3 C}{4}\right) \\
Z^{\prime}\left(\phi_{2}\right) & =-C \sin \left(\frac{\pi}{3}+3 C\right) \\
& \approx-C\left(\frac{\sqrt{3}}{2}+\frac{3 C}{2}\right) \\
g^{\prime}\left(\phi_{3}\right) & =0 .
\end{aligned}
$$

In the case of excitatory coupling $(C>0)$ we get $\phi_{1} \gtrsim-\pi / 3$ and $\phi_{2} \gtrsim \pi / 3$ and thus $0<Z^{\prime}\left(\phi_{1}\right)<$ $-Z^{\prime}\left(\phi_{2}\right)$. This means, that perturbations around $\phi_{1}$ increase less than perturbations at $\phi_{2}$ decrease and the splay state in the case of excitatory coupling would be stable. In the case of inhibitory coupling $(C<0)$, we get $\phi_{1} \lesssim-\pi / 3$ and $\phi_{2} \lesssim \pi / 3$ and thus $0<-Z^{\prime}\left(\phi_{1}\right)<Z^{\prime}\left(\phi_{2}\right)$. This means, that perturbations around $\phi_{1}$ decrease less than perturbations at $\phi_{2}$ increase and the splay state in the case of inhibitory coupling would be unstable.

This analysis should be extended to the rapid theta neuron model which hopefully provides more insight into the observed qualitatively different dynamics. The shape of the phase-response curve of the rapid theta neuron model (Fig. 3.2) exhibit a shift of the peak towards the spike at $\phi=\pi$. This suggests that for large AP onset rapidness the asynchronous splay state might become stable in all-to-all coupled networks. The critical value between an unstable and stable splay state might depend on the number of neurons in the networks as this determines the phases for which the splay state exists. 


\section{Appendix E}

\section{Propagator Method}

The propagator method is used to solve the inhomogeneous differential equation

$$
\dot{x}(t)=A(t) x+B(t)
$$

with the initial condition $x\left(t_{0}\right)$. The corresponding homogeneous equation has the solution (propagator):

$$
P\left(t, t^{\prime}\right)=\mathrm{e}^{\int_{t^{\prime}}^{t} A(\tau) \mathrm{d} \tau}
$$

Using the propagator, the inhomogeneous equation is solved with

$$
x(t)=P\left(t, t_{0}\right) x\left(t_{0}\right)+\int_{t_{0}}^{t} P\left(t, t^{\prime}\right) B\left(t^{\prime}\right) \mathrm{d} t^{\prime} .
$$





\section{References}

[1] B. Naundorf, F. Wolf, and M. Volgushev: "Unique features of action potential initiation in cortical neurons." Nature 440, 1060-1063 (2006).

[2] C. van Vreeswijk and H. Sompolinsky: "Chaos in neuronal networks with balanced excitatory and inhibitory activity." Science 274, 1724-1726 (1996).

[3] C. van Vreeswijk and H. Sompolinsky: "Chaotic balanced state in a model of cortical circuits." Neural Computation 10, 1321-1371 (1998).

[4] R. Zillmer, R. Livi, A. Politi, and A. Torcini: "Desynchronization in diluted neural networks.” Physical Review E 74, 036203 (2006).

[5] R. Zillmer, N. Brunel, and D. Hansel: "Very long transients, irregular firing, and chaotic dynamics in networks of randomly connected inhibitory integrate-and-fire neurons." Physical Review E 79, 031909 (2009).

[6] S. Jahnke, R.-M. Memmesheimer, and M. Timme: "Stable irregular dynamics in complex neural networks." Physical Review Letters 100, 048102 (2008).

[7] S. Jahnke, R.-M. Memmesheimer, and M. Timme: "How Chaotic is the Balanced State?" Frontiers in Computational Neuroscience 3, 13 (2009).

[8] M. Monteforte and F. Wolf: "Dynamical entropy production in spiking neuron networks in the balanced state." Physical Review Letters 105, 268104 (2010).

[9] R. R. Wang: "Yinyang." The Internet Encyclopedia of Philosophy (2006).

[10] B. D. Burns and A. C. Webb: "The Spontaneous Activity of Neurones in the Cat's Cerebral Cortex." Proceedings of the Royal Society B 194, 211-223 (1976).

[11] W. R. Softky and C. Koch: "The highly irregular firing of cortical cells is inconsistent with temporal integration of random EPSPs." The Journal of Neuroscience 13, 334-350 (1993).

[12] M. N. Shadlen and W. T. Newsome: "Noise, neural codes and cortical organization." Current Opinion in Neurobiology 4, 569-579 (1994).

[13] M. N. Shadlen and W. T. Newsome: "The variable discharge of cortical neurons: implications for connectivity, computation, and information coding." The Journal of Neuroscience 18, 3870-3896 (1998).

[14] Y. Shu, A. Hasenstaub, and D. A. McCormick: "Turning on and off recurrent balanced cortical activity." Nature 423, 288-293 (2003). 
[15] M. Wehr and A. M. Zador: "Balanced inhibition underlies tuning and sharpens spike timing in auditory cortex." Nature 426, 442-446 (2003).

[16] B. Haider, A. Duque, A. R. Hasenstaub, and D. A. McCormick: "Neocortical network activity in vivo is generated through a dynamic balance of excitation and inhibition." The Journal of Neuroscience 26, 4535-4545 (2006).

[17] M. Okun and I. Lampl: "Instantaneous correlation of excitation and inhibition during ongoing and sensory-evoked activities." Nature Neuroscience 11, 535-537 (2008).

[18] B. V. Atallah and M. Scanziani: "Instantaneous modulation of gamma oscillation frequency by balancing excitation with inhibition." Neuron 62, 566-577 (2009).

[19] L. C. Populin: "Anesthetics change the excitation/inhibition balance that governs sensory processing in the cat superior colliculus." The Journal of Neuroscience 25, 5903-5914 (2005).

[20] A. K. Magnusson, T. J. Park, M. Pecka, B. Grothe, and U. Koch: "Retrograde GABA signaling adjusts sound localization by balancing excitation and inhibition in the brainstem." Neuron 59, 125-137 (2008).

[21] R. W. Berg, A. Alaburda, and J. Hounsgaard: "Balanced inhibition and excitation drive spike activity in spinal half-centers." Science 315, 390-393 (2007).

[22] Z. F. Mainen and T. J. Sejnowski: "Reliability of spike timing in neocortical neurons." Science 268, 1503-1506 (1995).

[23] G. R. Holt, W. R. Softky, C. Koch, and R. J. Douglas: "Comparison of discharge variability in vitro and in vivo in cat visual cortex neurons." Journal of Neurophysiology 75, 18061814 (1996).

[24] M. Tsodyks and T. Sejnowski: "Rapid state switching in balanced cortical network models." Network 6, 111-124 (1995).

[25] T. W. Troyer and K. D. Miller: "Physiological gain leads to high ISI variability in a simple model of a cortical regular spiking cell." Neural Computation 9, 971-983 (1997).

[26] D. J. Amit and N. Brunel: "Model of global spontaneous activity and local structured activity during delay periods in the cerebral cortex." Cerebral Cortex 7, 237-252 (1997).

[27] D. Amit and N. Brunel: "Dynamics of a recurrent network of spiking neurons before and following learning." Network 8, 373-404 (1997).

[28] N. Brunel: "Dynamics of sparsely connected networks of excitatory and inhibitory spiking neurons." Journal of Computational Neuroscience 8, 183-208 (2000).

[29] A. Lerchner, C. Ursta, J. Hertz, M. Ahmadi, P. Ruffiot, and S. r. Enemark: "Response variability in balanced cortical networks." Neural Computation 18, 634-659 (2006).

[30] A. Renart, R. Moreno-Bote, X.-J. Wang, and N. Parga: "Mean-driven and fluctuation-driven persistent activity in recurrent networks." Neural Computation 19, 1-46 (2007). 
[31] A. Kumar, S. Schrader, A. Aertsen, and S. Rotter: "The high-conductance state of cortical networks." Neural Computation 20, 1-43 (2008).

[32] A. Renart, J. de la Rocha, P. Bartho, L. Hollender, N. Parga, A. Reyes, and K. D. Harris: "The asynchronous state in cortical circuits." Science 327, 587-590 (2010).

[33] J. Hertz: "Cross-correlations in high-conductance states of a model cortical network." Neural Computation 22, 427-447 (2010).

[34] B. Derrida, E. Gardner, and A. Zippelius: "An Exactly Solvable Asymmetric Neural Network Model." Europhysics Letters (EPL) 4, 167-173 (1987).

[35] A. Politi, R. Livi, G. L. Oppo, and R. Kapral: "Unpredictable Behaviour in Stable Systems." Europhysics Letters 22, 571-576 (1993).

[36] A. L. Hodgkin and A. F. Huxley: "A quantitative description of membrane current and its application to conduction and excitation in nerve." The Journal of Physiology 117, 500-544 (1952).

[37] B. Ermentrout: "Neural networks as spatio-temporal pattern-forming systems." Reports on Progress in Physics 61, 353-430 (1998).

[38] X. J. Wang and G. Buzsáki: "Gamma oscillation by synaptic inhibition in a hippocampal interneuronal network model." The Journal of Neuroscience 16, 6402-6413 (1996).

[39] J. A. Connor and C. F. Stevens: "Prediction of repetitive firing behaviour from voltage clamp data on an isolated neurone soma." The Journal of Physiology 213, 31-53 (1971).

[40] Y. Yu, Y. Shu, and D. A. McCormick: "Cortical action potential backpropagation explains spike threshold variability and rapid-onset kinetics." The Journal of Neuroscience $\mathbf{2 8}, \mathbf{7 2 6 0}$ 7272 (2008).

[41] D. A. McCormick, Y. Shu, and Y. Yu: "Neurophysiology: Hodgkin and Huxley model-still standing?" Nature 445, E1-E2 (2007).

[42] B. Naundorf, F. Wolf, and M. Volgushev: "Neurophysiology: Hodgkin and Huxley model still standing? (Reply)." Nature 445, E2-E3 (2007).

[43] W. Hu, C. Tian, T. Li, M. Yang, H. Hou, and Y. Shu: "Distinct contributions of Na(v)1.6 and $\mathrm{Na}(\mathrm{v}) 1.2$ in action potential initiation and backpropagation." Nature Neuroscience 12, 996-1002 (2009).

[44] I. A. Fleidervish, N. Lasser-Ross, M. J. Gutnick, and W. N. Ross: "Na+ imaging reveals little difference in action potential-evoked $\mathrm{Na}+$ influx between axon and soma." Nature Neuroscience 13, 852-860 (2010).

[45] C. A. Skarda and W. J. Freeman: "How brains make chaos in order to make sense of the world." Behavioral and Brain Sciences 10, 161-195 (2010).

[46] H. Sompolinsky, A. Crisanti, and H. Sommers: "Chaos in Random Neural Networks." Physical Review Letters 61, 259-262 (1988). 
[47] D. Hansel and H. Sompolinsky: "Chaos and synchrony in a model of a hypercolumn in visual cortex." Journal of Computational Neuroscience 3, 7-34 (1996).

[48] A. Banerjee: "On the sensitive dependence on initial conditions of the dynamics of networks of spiking neurons." Journal of Computational Neuroscience 20, 321-348 (2006).

[49] A. Banerjee, P. Seriès, and A. Pouget: "Dynamical constraints on using precise spike timing to compute in recurrent cortical networks." Neural Computation 20, 974-993 (2008).

[50] R. E. Mirollo and S. H. Strogatz: "Synchronization of Pulse-Coupled Biological Oscillators.” SIAM Journal on Applied Mathematics 50, 1645-1662 (1990).

[51] M. Tsodyks, I. Mitkov, and H. Sompolinsky: "Pattern of synchrony in inhomogeneous networks of oscillators with pulse interactions." Physical Review Letters 71, 1280-1283 (1993).

[52] L. F. Abbott and van Vreeswijk Carl: "Asynchronous states in networks of pulse-coupled oscillators.” Physical Review E 48, 1483-1490 (1993).

[53] C. van Vreeswijk: "Partial synchronization in populations of pulse-coupled oscillators." Physical Review E 54, 5522-5537 (1996).

[54] U. Ernst, K. Pawelzik, and T. Geisel: "Synchronization induced by temporal delays in pulsecoupled oscillators." Physical Review Letters 74, 1570-1573 (1995).

[55] U. Ernst, K. Pawelzik, and T. Geisel: "Delay-induced multistable synchronization of biological oscillators." Physical Review E 57, 2150-2162 (1998).

[56] M. Timme, F. Wolf, and T. Geisel: "Prevalence of unstable attractors in networks of pulsecoupled oscillators." Physical Review Letters 89, 154105 (2002).

[57] M. Timme, F. Wolf, and T. Geisel: "Coexistence of regular and irregular dynamics in complex networks of pulse-coupled oscillators.” Physical Review Letters 89, 258701 (2002).

[58] M. Timme, F. Wolf, and T. Geisel: "Unstable attractors induce perpetual synchronization and desynchronization." Chaos 13, 377-387 (2003).

[59] M. Denker, M. Timme, M. Diesmann, F. Wolf, and T. Geisel: "Breaking synchrony by heterogeneity in complex networks." Physical Review Letters 92, 074103 (2004).

[60] D. Z. Jin: "Fast convergence of spike sequences to periodic patterns in recurrent networks." Physical Review Letters 89, 208102 (2002).

[61] M. Timme, F. Wolf, and T. Geisel: "Topological speed limits to network synchronization." Physical Review Letters 92, 074101 (2004).

[62] M. Timme, T. Geisel, and F. Wolf: "Speed of synchronization in complex networks of neural oscillators: analytic results based on Random Matrix Theory." Chaos 16, 015108 (2006).

[63] M. Timme and F. Wolf: "The simplest problem in the collective dynamics of neural networks: is synchrony stable?" Nonlinearity 21, 1579-1599 (2008). 
[64] A. Zumdieck, M. Timme, T. Geisel, and F. Wolf: "Long chaotic transients in complex networks." Physical Review Letters 93, 244103 (2004).

[65] D. Zhou, A. V. Rangan, Y. Sun, and D. Cai: "Network-induced chaos in integrate-and-fire neuronal ensembles." Physical Review E 80, 031918 (2009).

[66] S. El Boustani and A. Destexhe: "Brain Dynamics At Multiple Scales: Can One Reconcile the Apparent Low-Dimensional Chaos of Macroscopic Variables With the Seemingly Stochastic Behavior of Single Neurons?" International Journal of Bifurcation and Chaos 20, 1687-1702 (2010).

[67] J.-P. Eckmann and D. Ruelle: "Ergodic theory of chaos and strange attractors." Reviews of Modern Physics 57, 617-656 (1985).

[68] G. Benettin, L. Galgani, A. Giorgilli, and J.-M. Strelcyn: "Lyapunov Characteristic Exponents for smooth dynamical systems and for hamiltonian systems; a method for computing all of them. Part 1: Theory." Meccanica 15, 9-20 (1980).

[69] G. Benettin, L. Galgani, A. Giorgilli, and J.-M. Strelcyn: "Lyapunov Characteristic Exponents for smooth dynamical systems and for hamiltonian systems; A method for computing all of them. Part 2: Numerical application.” Meccanica 15, 21-30 (1980).

[70] R. Shaw: "Strange Attractors, Chaotic Behaviour and Information Flow." Zeitschrift für Naturforschung 36A, 80-112 (1981).

[71] D. Ruelle: “An inequality for the entropy of differentiable maps.” Bulletin of the Brazilian Mathematical Society 9, 83-87 (1978).

[72] F. Ledrappier and L.-S. Young: "The Metric Entropy of Diffeomorphisms: Part I: Characterization of Measures Satisfying Pesin's Entropy Formula and Part II: Relations between Entropy, Exponents and Dimension.” The Annals of Mathematics 122, 509-539 (1985).

[73] F. Ledrappier: "Some relations between dimension and Lyapounov exponents." Communications in Mathematical Physics 81, 229-238 (1981).

[74] J. L. Kaplan and J. A. Yorke: "Preturbulence: A regime observed in a fluid flow model of Lorenz." Communications in Mathematical Physics 67, 93-108 (1979).

[75] P. Grassberger: "Generalized dimensions of strange attattractors." Physics Letters 97, 227230 (1983).

[76] J. D. Farmer: "Information dimension and the probabilistic structure of chaos." Zeitschrift für Naturforschung 37, 1304-1325 (1982).

[77] R. W. Leven, B.-P. Koch, and B. Pompe: Chaos in dissipativen Systemen (Berlin, Akademie Verlag, 1994). ISBN 3055016416.

[78] E. Schneidman, M. J. Berry, R. Segev, and W. Bialek: "Weak pairwise correlations imply strongly correlated network states in a neural population." Nature 440, 1007-1012 (2006). 
[79] J. Shlens, G. D. Field, J. L. Gauthier, M. I. Grivich, D. Petrusca, A. Sher, A. M. Litke, and E. J. Chichilnisky: "The structure of multi-neuron firing patterns in primate retina." The Journal of Neuroscience 26, 8254-8266 (2006).

[80] A. Tang, D. Jackson, J. Hobbs, W. Chen, J. L. Smith, H. Patel, A. Prieto, D. Petrusca, M. I. Grivich, A. Sher, P. Hottowy, W. Dabrowski, A. M. Litke, and J. M. Beggs: "A maximum entropy model applied to spatial and temporal correlations from cortical networks in vitro." The Journal of Neuroscience 28, 505-518 (2008).

[81] I. E. Ohiorhenuan, F. Mechler, K. P. Purpura, A. M. Schmid, Q. Hu, and J. D. Victor: "Sparse coding and high-order correlations in fine-scale cortical networks." Nature 466, 617-621 (2010).

[82] F. Azhar and W. Bialek: "When are correlations strong?" arXiv 1012, 5987 (2010).

[83] G. B. Ermentrout and N. Kopell: "Parabolic Bursting in an Excitable System Coupled with a Slow Oscillation.” SIAM Journal on Applied Mathematics 46, 233-253 (1986).

[84] B. Ermentrout: “Type I membranes, phase resetting curves, and synchrony." Neural Computation 8, 979-1001 (1996).

[85] B. S. Gutkin and G. B. Ermentrout: "Dynamics of membrane excitability determine interspike interval variability: a link between spike generation mechanisms and cortical spike train statistics." Neural Computation 10, 1047-1065 (1998).

[86] P. Ashwin and M. Timme: "Unstable attractors: existence and robustness in networks of oscillators with delayed pulse coupling." Nonlinearity 18, 2035-2060 (2005).

[87] R. M. Smeal, G. B. Ermentrout, and J. A. White: "Phase-response curves and synchronized neural networks." Philosophical Transactions of the Royal Society of London. Series B $\mathbf{3 6 5}$, 2407-2422 (2010).

[88] N. Fourcaud-Trocmé, D. Hansel, C. van Vreeswijk, and N. Brunel: "How spike generation mechanisms determine the neuronal response to fluctuating inputs." The Journal of Neuroscience 23, 11628-11640 (2003).

[89] S. Gerschgorin: "Über die Abgrenzung der Eigenwerte einer Matrix." Izvestija Akademii Nauk SSSR Serija Matematika 7, 749-754 (1931).

[90] C. Kirst, T. Geisel, and M. Timme: "Sequential desynchronization in networks of spiking neurons with partial reset." Physical Review Letters 102, 068101 (2009).

[91] T. Yokozawa, D. Takahashi, and T. Boku: "Efficient parallel implementation of classical Gram-Schmidt orthogonalization using matrix multiplication." In Parallel Matrix Algorithms and Applications (PMAA'06), pages 37-38 (2006).

[92] D. Golomb and D. Hansel: "The number of synaptic inputs and the synchrony of large, sparse neuronal networks." Neural Computation 12, 1095-1139 (2000).

[93] S. Panzeri, R. S. Petersen, S. R. Schultz, M. Lebedev, and M. E. Diamond: "The role of spike timing in the coding of stimulus location in rat somatosensory cortex." Neuron 29, 769-777 (2001). 
[94] A. Borst and F. E. Theunissen: "Information theory and neural coding." Nature Neuroscience 2, 947-957 (1999).

[95] V. Braitenberg and A. Schüz: Cortex: Statistics and Geometry of Neuronal Connectivity. 81 (Springer-Verlag, 1998). ISBN 3540638164.

[96] F. Ginelli, P. Poggi, A. Turchi, H. Chaté, R. Livi, and A. Politi: "Characterizing dynamics with covariant Lyapunov vectors.” Physical Review Letters 99, 130601 (2007).

[97] W. H. Press, S. A. Teukolsky, W. T. Vetterling, and B. P. Flannery: Numerical Recipes in C (2nd Ed.): The Art of Scientific Computing (Cambridge University Press, New York, USA, 1992). ISBN 0-521-43108-5.

[98] S. Shoham, D. H. O'Connor, and R. Segev: "How silent is the brain: is there a "dark matter" problem in neuroscience?" Journal of Comparative Physiology A 192, 777-784 (2006).

[99] P. Hiemeyer: "Mean field theory of firing rate heterogeneity in balanced neural networks: On silent neurons, the dark matter of the brain." Diploma Thesis (2009).

[100] N. Brunel and V. Hakim: "Fast global oscillations in networks of integrate-and-fire neurons with low firing rates." Neural Computation 11, 1621-1671 (1999).

[101] M. London, A. Roth, L. Beeren, M. Häusser, and P. E. Latham: "Sensitivity to perturbations in vivo implies high noise and suggests rate coding in cortex." Nature 466, 123-127 (2010).

[102] D. Huber, L. Petreanu, N. Ghitani, S. Ranade, T. Hromádka, Z. Mainen, and K. Svoboda: "Sparse optical microstimulation in barrel cortex drives learned behaviour in freely moving mice." Nature 451, 61-64 (2008).

[103] M. Brecht, M. Schneider, B. Sakmann, and T. W. Margrie: "Whisker movements evoked by stimulation of single pyramidal cells in rat motor cortex." Nature 427, 704-710 (2004).

[104] A. R. Houweling and M. Brecht: "Behavioural report of single neuron stimulation in somatosensory cortex." Nature 451, 65-68 (2008).

[105] N. Bertschinger and T. Natschläger: "Real-time computation at the edge of chaos in recurrent neural networks." Neural Computation 16, 1413-1436 (2004).

[106] R. W. Friedrich and G. Laurent: "Dynamic optimization of odor representations by slow temporal patterning of mitral cell activity.” Science 291, 889-894 (2001).

[107] R. W. Friedrich: "Mechanisms of odor discrimination: neurophysiological and behavioral approaches." Trends in Neurosciences 29, 40-47 (2006).

[108] W. Maass, T. Natschläger, and H. Markram: "Real-time computing without stable states: a new framework for neural computation based on perturbations." Neural Computation 14, 2531-2560 (2002).

[109] H. Jaeger: "The "echo state" approach to analysing and training recurrent neural networks." GMD Report 148 (2001). 
[110] D. Ruelle: "Large volume limit of the distribution of characteristic exponents in turbulence." Communications in Mathematical Physics 87, 287-302 (1982).

[111] M. Cross and P. Hohenberg: "Pattern formation outside of equilibrium." Reviews of Modern Physics 65, 851-1112 (1993).

[112] L. A. Bunimovich and Y. G. Sinai: "Spacetime chaos in coupled map lattices." Nonlinearity 1, 491-516 (1988).

[113] K. Kaneko: "Pattern dynamics in spatiotemporal chaos Pattern selection, diffusion of defect and pattern competition intermettency." Physica D 34, 1-41 (1989).

[114] D. Hansel and H. Sompolinsky: "Solvable model of spatiotemporal chaos." Physical Review Letters 71, 2710-2713 (1993).

[115] D. Hansel and G. Mato: "Asynchronous states and the emergence of synchrony in large networks of interacting excitatory and inhibitory neurons." Neural Computation 15, 1-56 (2003).

[116] N. Brunel and X.-J. Wang: "What determines the frequency of fast network oscillations with irregular neural discharges? I. Synaptic dynamics and excitation-inhibition balance." Journal of Neurophysiology 90, 415-430 (2003).

[117] C. Geisler, N. Brunel, and X.-J. Wang: "Contributions of intrinsic membrane dynamics to fast network oscillations with irregular neuronal discharges." Journal of Neurophysiology 94, 4344-4361 (2005).

[118] Y. Aviel, C. Mehring, M. Abeles, and D. Horn: "On embedding synfire chains in a balanced network." Neural Computation 15, 1321-1340 (2003).

[119] T. P. Vogels and L. F. Abbott: "Signal propagation and logic gating in networks of integrateand-fire neurons." The Journal of Neuroscience 25, 10786-10795 (2005).

[120] S. Ganguli, D. Huh, and H. Sompolinsky: "Memory traces in dynamical systems." Proceedings of the National Academy of Sciences of the United States of America 105, 18970-18975 (2008).

[121] S. Schrader, M. Diesmann, and A. Morrison: "A compositionality machine realized by a hierarchic architecture of synfire chains." Frontiers in Computational Neuroscience 4, 154 (2011).

[122] A. Tonnelier, H. Belmabrouk, and D. Martinez: "Event-driven simulations of nonlinear integrate-and-fire neurons." Neural Computation 19, 3226-3238 (2007).

[123] J.-H. Schleimer and M. Stemmler: "Coding of information in limit cycle oscillators." Physical Review Letters 103, 248105 (2009).

[124] R. Ben-Yishai: "Theory of Orientation Tuning in Visual Cortex." Proceedings of the National Academy of Sciences 92, 3844-3848 (1995).

[125] D. Sussillo and L. F. Abbott: "Generating coherent patterns of activity from chaotic neural networks." Neuron 63, 544-557 (2009). 
[126] L. Badel, S. Lefort, R. Brette, C. C. H. Petersen, W. Gerstner, and M. J. E. Richardson: "Dynamic I-V curves are reliable predictors of naturalistic pyramidal-neuron voltage traces." Journal of Neurophysiology 99, 656-666 (2008).

[127] W. Wei and F. Wolf: "Spike onset dynamics and response speed in neuronal populations." Physical Review Letters 106, 088102 (2011).

[128] P. Goel and B. Ermentrout: "Synchrony, stability, and firing patterns in pulse-coupled oscillators." Physica D 163, 191-216 (2002).

[129] C. C. Canavier and S. Achuthan: "Pulse coupled oscillators and the phase resetting curve." Mathematical Biosciences 226, 77-96 (2010).

[130] C. Kirst and M. Timme: "Partial Reset in Pulse-Coupled Oscillators." SIAM Journal on Applied Mathematics 70, 2119-2149 (2010).

[131] C. Kirst: "Kirst conjecture.” private communication . 



\section{List of Figures}

1.1 The balanced state in densely connected networks. . . . . . . . . . . . . . . 4

1.2 Firing rate functions of type I and type II excitable membranes. . . . . . . . . . . . 7

1.3 Standard neuron models of noncortical neurons. . . . . . . . . . . . . . . . 7

1.4 Action potential generation in cortical neurons. . . . . . . . . . . . . . . 9

1.5 Information loss in chaotic systems. . . . . . . . . . . . . . . . . . . 14

$1.6 \quad$ Weak pairwise correlation imply strongly correlated network states. . . . . . . . . 15

2.1 The theta neuron model. . . . . . . . . . . . . . . . . . . . . 28

2.2 Comparison of mean Lyapunov exponent in random matrix approximations and from numerical simulations in balanced theta neuron networks. . . . . . . . . . . . 32

2.3 Convergence of Lyapunov spectra versus time in inhibitory networks. . . . . . . . 33

2.4 Convergence of largest Lyapunov exponent in inhibitory networks versus average number of spikes per neuron. . . . . . . . . . . . . . . . . 34

2.5 Convergence of largest Lyapunov exponent in excitatory-inhibitory networks versus average number of spikes per neuron. $\ldots \ldots \ldots \ldots$. . . . . . . . 34

2.6 Identical firing characteristics in inhibitory networks and excitatory-inhibitory networks in the balanced state. . . . . . . . . . . . . . . 36

2.7 Transition from asynchronous irregular state to synchronous irregular state in in-

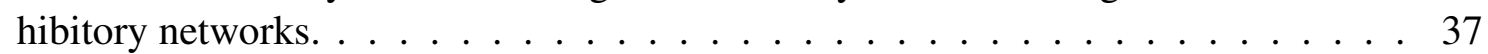

2.8 Transition from asynchronous irregular state to synchronous regular state in excitatory-

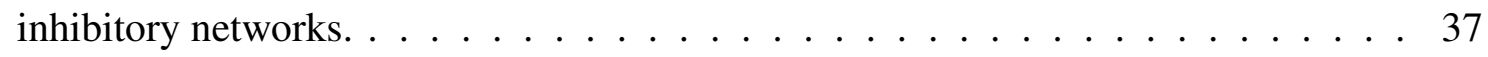

2.9 Phase diagrams of excitatory-inhibitory networks for three different ratios $\eta=$

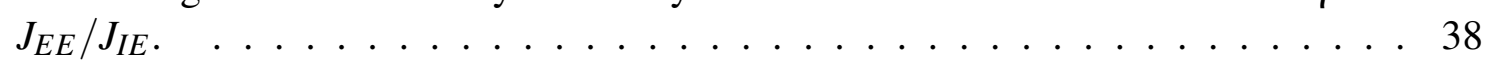

2.10 Extensive chaos in balanced inhibitory networks of theta neurons. . . . . . . . . 39

2.11 Deterministic chaos in balanced inhibitory networks for varied average firing rates. 39

2.12 Deterministic chaos in balanced inhibitory networks for varied coupling strengths. 40

2.13 Deterministic chaos in inhibitory networks for varied number of presynaptic neurons. 40

2.14 Chaotic dynamics in balanced excitatory-inhibitory networks while activating the excitatory-inhibitory feedback loops. . . . . . . . . . . . . . . 41

2.15 Stable dynamics during transition from asynchronous irregular to synchronous regular state for strong excitatory couplings. . . . . . . . . . . . . . . . . 42

2.16 Chaotic dynamics in balanced excitatory-inhibitory networks for varied number of

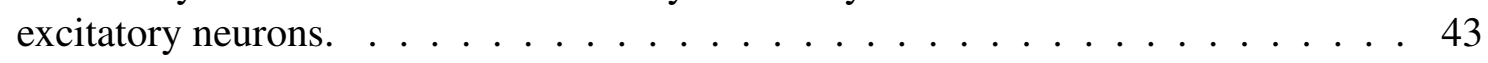

2.17 Chaotic dynamics in balanced excitatory-inhibitory networks for varied populationaveraged firing rates. $\ldots \ldots \ldots \ldots \ldots 4 \ldots \ldots \ldots$

2.18 Chaotic dynamics in balanced excitatory-inhibitory networks for varied number of

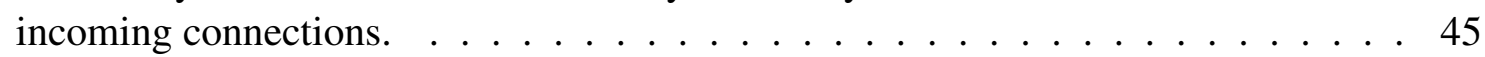


2.19 Extensive chaos in balanced excitatory-inhibitory networks for varied network sizes. 45

2.20 Temporal network chaos in balanced inhibitory networks. . . . . . . . . . . 46

2.21 Temporal network chaos in excitatory-inhibitory balanced theta neuron networks. . 46

2.22 Average participation ratio versus network size in balanced theta neuron networks with different average firing rates. $\ldots \ldots \ldots \ldots$. . . . . . . . . . . . 47

2.23 Covariant Lyapunov vectors in balanced theta neuron networks. . . . . . . . . . . 50

2.24 Minimal angle between stable and unstable manifold. . . . . . . . . . . . . . . . 50

$3.1 \quad$ Rapid theta neuron model. . . . . . . . . . . . . . . . . . . . . 54

3.2 Phase-transition curve, phase-response and infinitesimal phase-response of the rapidtheta neuron model with AP onset rapidness. . . . . . . . . . . . . . . . . 58

$3.3 \quad$ Characteristics of the balanced state in rapid theta neuron networks. . . . . . . . . 59

3.4 Phase diagrams of excitatory-inhibitory networks for different $\eta=J_{E E} / J_{I E}$. . . . 60

3.5 Extensive dynamics in balanced inhibitory networks of rapid theta neurons. . . . . 61

3.6 Critical AP onset rapidness between stable and chaotic dynamics in balanced in-

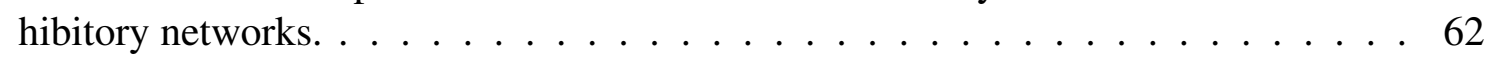

$3.7 \quad$ AP rapidness strongly influences the dynamics of balanced inhibitory networks. . . 63

3.8 Dynamics of dense networks for different AP rapidness and varied number of

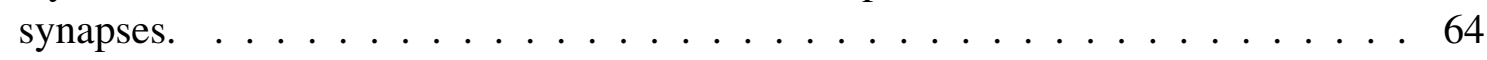

3.9 Transition from asynchronous irregular to synchronous irregular state shifted with increasing on AP rapidness. . . . . . . . . . . . . . . . . . . 65

3.10 Different scaling regimes of largest Lyapunov exponent with number of synapses explain the peak in largest Lyapunov exponent versus AP onset rapidness. . . . . . 65

3.11 Dynamics of excitatory-inhibitory networks with different AP onset rapidness, while activating the excitatory-inhibitory feedback loops. . . . . . . . . . . . . 67

3.12 AP onset rapidness strongly influences the dynamics of excitatory-inhibitory balanced networks with excitatory-inhibitory feedback strength. . . . . . . . . . . 68

3.13 Comparison of AP onset rapidness-influence on the dynamics of balanced neuron

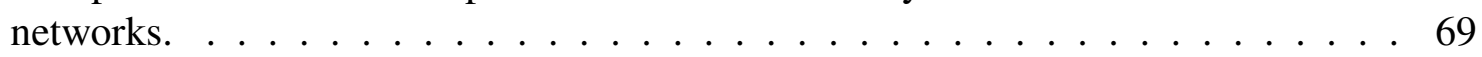

3.14 Critical AP onset rapidness at which the networks are at the edge of chaos. . . . . . 69

3.15 Extensive chaos in excitatory-inhibitory balanced networks for varied network sizes. 70

3.16 Dynamics of excitatory-inhibitory balanced networks for different AP onset rapidness and varied number of synapses. . . . . . . . . . . . . . 71

3.17 Occurrence of transition from asynchronous irregular to synchronous irregular state depends on AP rapidness. . . . . . . . . . . . . . . . . 71

3.18 Scaling of the average participation ratio in rapid theta neuron networks. . . . . . . 72

3.19 Comparison of correlation coefficient and Pearson coefficient in theta neuron net-

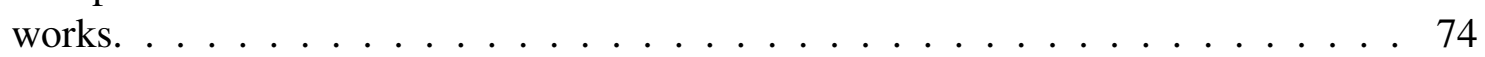

3.20 Averaged pairwise spike correlation of 50 neurons in three different firing rate

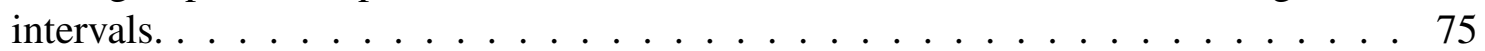

3.21 Pairwise spike correlation of neurons with approximately the average firing rate in balanced inhibitory networks. . . . . . . . . . . . . . 76

3.22 Average and standard deviation of the distributions of Pearson coefficient in rapid

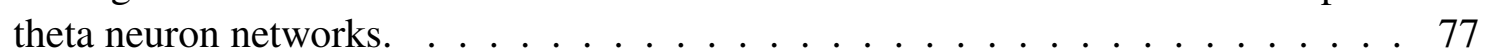

3.23 Distribution of pairwise spike correlations of 150 randomly chosen neurons in balanced inhibitory networks. . . . . . . . . . . . . 78 
3.24 Entangled statistics in rapid theta neuron networks. . . . . . . . . . . . . . 80

4.1 The correlated leaky integrate and fire model. . . . . . . . . . . . . . . . . . . 84

4.2 Firing statistics in inhibitory correlated integrate and fire neuron networks with different synaptic time constants. . . . . . . . . . . . . . 95

4.3 Phase diagrams of excitatory-inhibitory networks for different ratios. $\eta=J_{E E} / J_{I E}$. 96

4.4 Extensive dynamics in balanced inhibitory networks of correlated integrate and

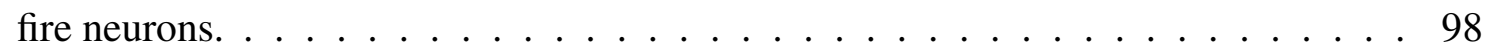

4.5 Dynamics for different synaptic time constants and $\delta$ pulse coupling (LIF). . . . . 99

4.6 Dynamics for varied coupling strength. . . . . . . . . . . . . . . . . . . 99

4.7 Dynamics for varied number of synapses. . . . . . . . . . . . . . . . . . . . 100

4.8 Dynamics for varied network-averaged firing rate. . . . . . . . . . . . . . . . 101

4.9 Dynamics of balanced excitatory-inhibitory networks while activating the excitatoryinhibitory feedback loops. . . . . . . . . . . . . . . . . 101

4.10 Dynamics for different synaptic time constants. . . . . . . . . . . . . . . 102

4.11 Dynamics for varied ratio of the number of excitatory to inhibitory neurons. . . . . 102

5.1 Membrane potential distributions and mean Lyapunov exponents from meanfield

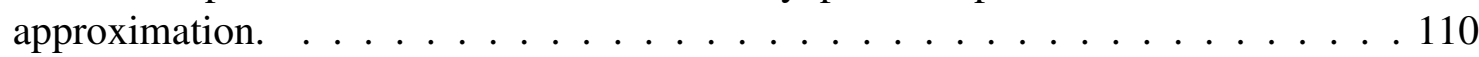

5.2 The balanced state in inhibitory LIF networks. . . . . . . . . . . . . . . . . 111

5.3 Extensive stable dynamics with respect to infinitesimal perturbations. . . . . . . . 113

5.4 Invariance of Lyapunov spectra for large network sizes. . . . . . . . . . . . . . . 113

5.5 Weak firing rate response after single spike failure. . . . . . . . . . . . . . . 114

5.6 Sensitivity to single spike failures. . . . . . . . . . . . . . . 116

5.7 Sensitivity to finite-size perturbations. . . . . . . . . . . . . . . 117

5.8 Flux tube radius estimation for different network sizes, connectivities and networkaveraged firing rates. . . . . . . . . . . . . . . . . . . . 118

5.9 Probability for single synaptic failures to induce switch to a different exponentially separating flux tube. . . . . . . . . . . . . . . . . . 119

5.10 Sensitivity to single spike failures in networks with (almost) instantaneous AP

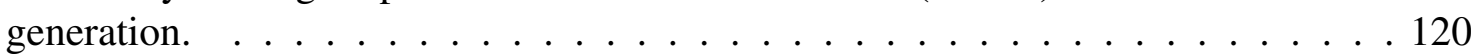

5.11 Sensitivity to single spike failures in networks with high AP onset rapidness. . . . . 121

5.12 Sensitivity to single spike failures in networks with low AP onset rapidness. . . . . 122

A.1 Integrate and fire neuron models. . . . . . . . . . . . . . . . . 136

A.2 Tonic firing rates versus suprathreshold input current (FI-curves) of different integrate and fire models. . . . . . . . . . . . . . . . . . . . . 139

A.3 Experimentally obtained membrane current versus membrane potential (IV-curve) of a cortical neuron and the fit to the EIF model from Ref. [126]. . . . . . . . . . . 140

A.4 Tonic firing rates versus suprathreshold input current (FI-curves) of the EIF model fit to the Wang-Buzsáki model $[88]$ and to a cortical neuron $[126]$. . . . . . . . . . 141

D.1 Example phase-response curve and phase-transition curve of type I excitable neurons. 149 


\section{Statement of Originality}

I hereby declare that I prepared this PhD thesis entitled "Chaotic Dynamics in Networks of Spiking Neurons in the Balanced State" on my own and with no other sources and aids than quoted.

Göttingen, 31th March 2011

Michael Monteforte 


\section{Acknowledgment}

I would like to thank Theo Geisel for creating and keeping the scientific oasis Max Planck Institute for Dynamics and Self-Organization, Department of Nonlinear Dynamics alive. Only this environment with a one of a kind atmosphere, and all the outstanding scientists it shelters have made this thesis possible in its final form. I also want to thank Theo for his constant interest and comments on this project and for introducing me to the fascinating field of nonlinear dynamics and chaos.

I want to thank Fred Wolf for being the spiritual source of this thesis. Only his guidance and the constant fountain of new ideas and solutions to my questions have led to the success of this thesis. Thanks Fred, for introducing me to the amazing field of theoretical neuroscience and for always supporting me scientifically and personally under any circumstances.

There are so many more people that contributed scientifically to this thesis. I want to specially thank Eberhard Bodenschatz for interesting discussions and providing new aspects in my thesis committee meetings, Demian Battaglia for enlighten me about the Shannon information theory, and Christoph Kirst, Wolfgang Keil, Jakob Metzger, Peter Hiemeyer, Harold Gutch, Christian Bick, Georg Martius, Anna Martius, Max Puelma Touzel, Wei Wei, Tatjana Tchumatchenko, Frank van Bussel, Oliver Bendix, Andreas Neef, Ragnar Fleischmann, Michael Hermann, Marc Timme, Raoul-Martin Memmesheimer, Sven Jahnke, Carl van Vreeswijk, Haim Sompolinsky, Sarah Solla, Predrac Cvitanović and many more for helpful comments and discussions.

I also want to thank Denny Fliegner for setting up the high-performance cluster and providing the computational power that was necessary for this thesis. In this respect, I also want to thank Yorck-Fabian Beensen, Marcus Schwamberger, Barbara Guichemer and Hecke Schrobsdorff for keeping the servers and computers run sound and smooth. For their great administrative work and always welcomed nonscientific conversations, I would like to thank Tobias Niemann, Regina Wunderlich, Katharina Jeremias, Regina Wunderlich, Tanja Gindele, Daniela Zarling, Ayse Bolik and Victoryia Novak.

I have already mentioned the amazing environment in which I was lucky enough to spend the past few years to work on this thesis. I want to thank everyone from the MPIDS who contributed to this unique atmosphere. Special thanks in this regard go to the think tank: Wolfgang, Jakob and Christoph. You were always a source of inspiration and have given me the opportunity to be part of a one time only performance. I want to thank Georg for helpful tips when it came to computer problems and possible code-optimizations, for his always optimistic and happy attitude and a great time in and outside of the institute. Similarly, I want to thank Harold and Christian who additionally tried to improve my precision when it comes to mathematics. Thanks also to Jens for his important contribution of enticing me to Göttingen, as well as my friends Jörn, Dave, Heiko, Juliane, Christof, Annette, Thomas, Steffen, Matthias, Stefan F., Stefan H., Stefan K., and many more for the balance between work and life.

Last but not least, the most important people I want to thank are my wonderful wife Michelle, my parents, and my sister and her family who always support me without reserve. I especially want to thank Michelle for her constant motivation, the necessary compensation and simply the best time in my life. 



\section{List of Symbols}

$\bar{v} \quad$ The network-averaged firing rate.

$\chi \quad$ The synchrony measure, Eq. (2.50).

$\eta \quad$ The ratio of inter-population excitatory coupling, Eq. (2.31).

$\lambda_{i} \quad$ The $i$-th Lyapunov exponent.

D The single spike Jacobian.

$\omega \quad$ The constant phase velocity of a neuron.

$\phi \quad$ The phase of a neuron.

$\tau_{\mathrm{I}} \quad$ The synaptic time constant.

$\tau_{\mathrm{m}} \quad$ The membrane time constant.

$\varepsilon \quad$ The excitatory-inhibitory feedback loop strength, Eq. 2.31.

cv The coefficient of variation, Eq. 2.49).

$D \quad$ The attractor dimension, Eq. (1.6).

$D_{\phi} \quad$ The phase-distance between trajectories.

$H \quad$ The entropy production rate, Eq. (1.5).

$I_{0} \quad$ The strength of the constant external currents.

$I_{T} \quad$ The rheobase current.

$J_{0} \quad$ The strength of the synaptic coupling.

$K \quad$ The average number of synapses per neuron.

$N \quad$ The number of neurons in the networks.

$N_{E} \quad$ The number of excitatory neurons.

$N_{I} \quad$ The number of inhibitory neurons.

$r \quad$ The action potential onset rapidness.

$V \quad$ The membrane potential (voltage) of a neuron.

$Z(\phi)$ The phase-response curve.

AP Abbreviation for action potential.

cLIF The correlated leaky integrate and fire model.

LIF The leaky integrate and fire model. 